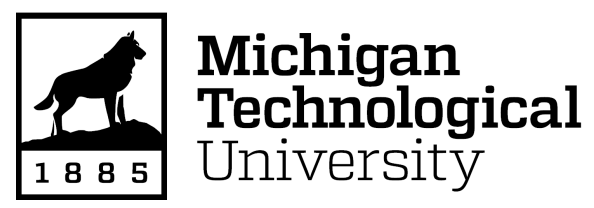

Michigan Technological University Digital Commons @ Michigan Tech

Dissertations, Master's Theses and Master's Reports

2018

\title{
A New Technique to Determine Accommodation Coefficients of Cryogenic Propellants
}

Kishan Bellur

Michigan Technological University, ksbellur@mtu.edu

Copyright 2018 Kishan Bellur

\section{Recommended Citation}

Bellur, Kishan, "A New Technique to Determine Accommodation Coefficients of Cryogenic Propellants", Open Access Dissertation, Michigan Technological University, 2018.

https://doi.org/10.37099/mtu.dc.etdr/647

Follow this and additional works at: https://digitalcommons.mtu.edu/etdr

Part of the Aerodynamics and Fluid Mechanics Commons, and the Heat Transfer, Combustion Commons 


\title{
A NEW TECHNIQUE TO DETERMINE ACCOMMODATION COEFFICIENTS OF CRYOGENIC PROPELLANTS
}

By

Kishan S. Bellur

\begin{abstract}
A DISSERTATION
Submitted in partial fulfillment of the requirements for the degree of DOCTOR OF PHILOSOPHY

In Mechanical Engineering - Engineering Mechanics

MICHIGAN TECHNOLOGICAL UNIVERSITY
\end{abstract}

2018

(C) 2018 Kishan S. Bellur 

This dissertation has been approved in partial fulfillment of the requirements for the Degree of DOCTOR OF PHILOSOPHY in Mechanical Engineering - Engineering Mechanics.

Department of Mechanical Engineering - Engineering Mechanics

Dissertation Co-advisor: Dr. Jeffrey Allen

Dissertation Co-advisor: Dr. Chang Kyoung Choi

Committee Member: Dr. Gregory Odegard

Committee Member: Dr. Daniel Hussey

Department Chair: Dr. William Predebon 



\section{Contents}

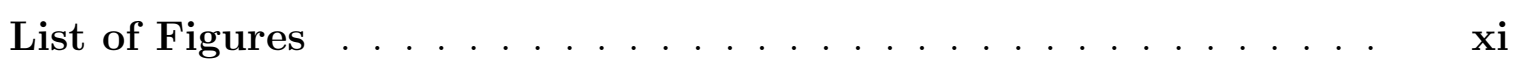

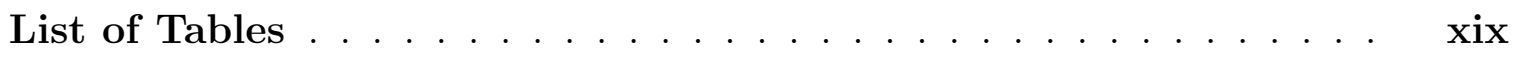

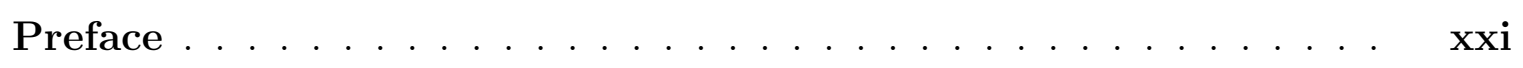

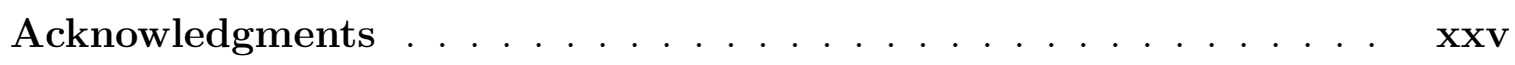

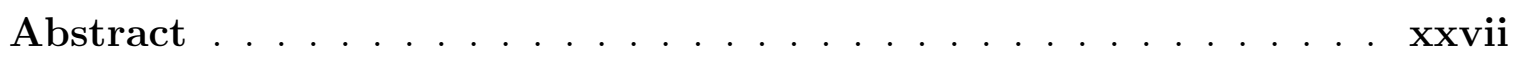

1 Introduction . . . . . . . . . . . . . . . . . . . . . . . 1

2 A new experiment for investigating evaporation and condensation

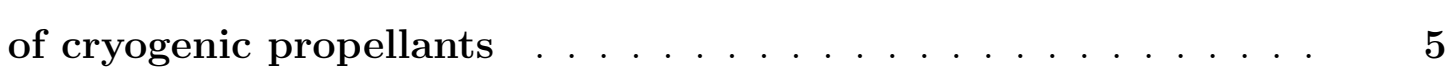

$2.1 \quad$ Neutron Imaging Experiments . . . . . . . . . . . . . . . . . 12

2.2 Thermal Modeling of Test Cells . . . . . . . . . . . . . . 20

2.3 Liquid-Vapor Interface Shape Determination . . . . . . . . . . . 23

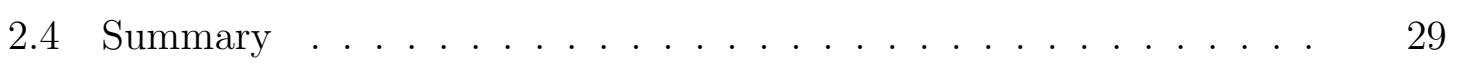


3 Visualization of the Evaporation and Condensation Phenomena in Cryogenic Propellants ................... 31

3.1 Introduction . . . . . . . . . . . . ..... 31

3.1.1 Neutron Imaging . . . . . . . . . . . . . . . . 34

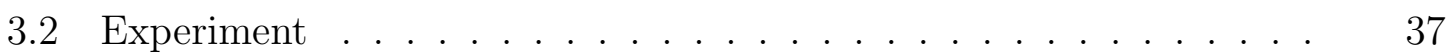

3.2.1 Test setup ............................... 37

3.2 .2 Imaging setup . . . . . . . . . . . . . . 40

3.3 Image Preprocessing . . . . . . . . . . . . . . . . 42

3.3.1 Liquid-Wall interface . . . . . . . . . . . . . . 43

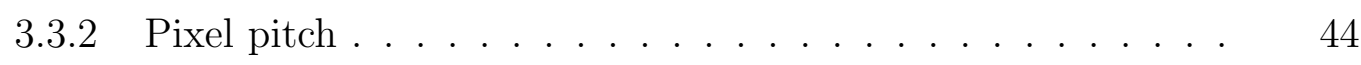

3.3.3 Image Rotation . . . . . . . . . . . . . . . 45

3.3.4 Deconvolution ................... 46

3.4 Image Analysis . . . . . . . . . . . . . . . . 46

3.4.1 Interface Tracking Method . . . . . . . . . . . 46

3.4.2 Optical density method .................. 52

3.5 Results and Discussion . . . . . . . . . . . . . . . . 56

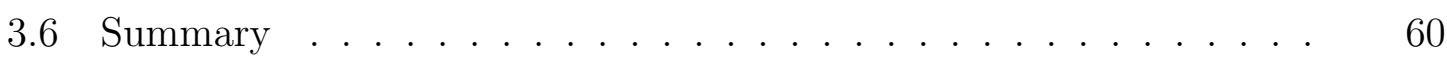

4 Determining solid-fluid interface temperature distribution during phase change ............................ 63

4.1 Introduction . . . . . . . . . . . . . . . . . . 63

4.2 Cryogenic Phase-Change Experiments . . . . . . . . . . . 66 
4.3 Transient Thermal Modeling of Cryostat . . . . . . . . . . . .

4.4 Contact Resistances . . . . . . . . . . . . . . . . . .

4.5 Determining Contact Resistances from Dry Cell Thermal Cycling .

4.6 Thermal Modeling for Phase Change Experiments . . . . . . . . . .

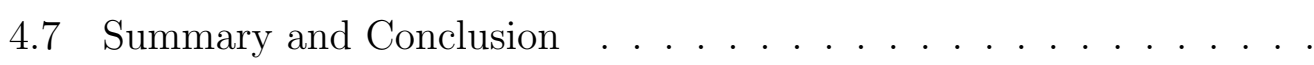

5 A combined experimental and multi-scale modeling approach to determine accommodation coefficients of cryogenic propellants .

5.1 Kinetic Model of Phase Change . . . . . . . . . . . . . . .

5.2 Interface Curvature . . . . . . . . . . . . . . .

5.3 Opportunity with Cryogenic Propellants . . . . . . . . . . .

5.4 Neutron imaging experiments

5.4.1 Thermal modeling of experiments .............

5.4.2 Macro-scale model of experiments . . . . . . . . . . . .

5.5 Transition film model . . . . . . . . . . . . . . . .

5.6 Computing the Accommodation Coefficient . . . . . . . . . . . .

5.7 Accommodation Coefficients of $\mathrm{LH}_{2} \ldots \ldots \ldots$

5.8 Summary and Conclusion . . . . . . . . . . . . .

6.1 Accommodation coefficients of methane . . . . . . . . . . . .

6.2 Effect of non-condensible gases . . . . . . . . . . . . . .

6.3 High resolution temperature sensors . . . . . . . . . . . . . 
6.4 Effect of disjoining pressure ................. 131

6.5 Testing of assumptions ..................... 132

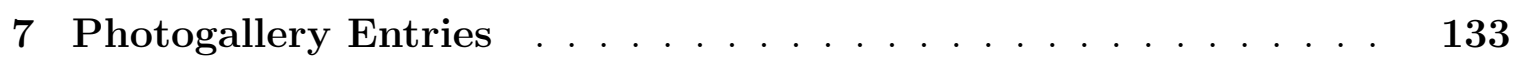

References ........................... 139

A Copyight Permission ..................... 163

B Code ............................... 173

B.1 YL_fit.m . . . . . . . . . . . . . . . . . 173

B.2 alpha_finder.m . . . . . . . . . . . . . . . 177

B.3 const_ac_mdot.m . . . . . . . . . . . . . . . . . 184

B.4 curvaturek.m ......................... 184

B.5 density.m ............................ 185

B.6 diff_eq.m . . . . . . . . . . . . . . . . . 186

B.7 disjoining.m . . . . . . . . . . . . . . . . . 191

B.8 externalHeatFlux.m . . . . . . . . . . . . . . . 191

B.9 fluidconst.m . . . . . . . . . . . . . . . . . . . 192

B.10 kTix.m . . . . . . . . . . . . . . . . 194

B.11 kTix.m . . . . . . . . . . . . . . . . . . 199

B.12 macro_model.m ...................... 204

B.13 sideWallTemperature.m ................... 212

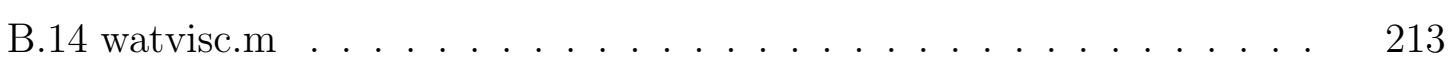


B.15 surf_ten.m . . . . . . . . . . . . . . . .

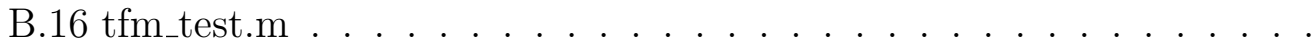

B.17 vapor_pressure.m . . . . . . . . . . . . . . . . . 218 



\section{List of Figures}

2.1 Extended meniscus with regions delineated by normal stress components. . . . . . . . . . . . . . . . .

2.2 Overview of experiments conducted at the NIST Neutron Imaging Facility (NIF). (a) Neutron Imaging Facility with cryostat in beam line. (b) Cryostat with test cell installed. (c) Location of copper block used for heating and cooling the test cell and helium gas in the sample well. (d) Sample holder with 10-mm test cell attached. (e) Cutaway view of the $10 \mathrm{~mm}$ diameter test cell and lid.

2.3 (a) Cutaway view of conical test cell. The smaller cylinder is $5 \mathrm{~mm}$ diameter and the larger cylinder is $30 \mathrm{~mm}$ diameter. The conical section has a 10 degree slope from the horizontal. (b) Liquid hydrogen in an aluminum 6061 test cell. A silicon diode sensor attached to the $5 \mathrm{~mm}$ diameter section is faintly visible. The flat hydrogen interface in the conical portion indicates the presence of a contact angle of approximately 10 degrees. (c) Normalized image emphasizing liquid hydrogen

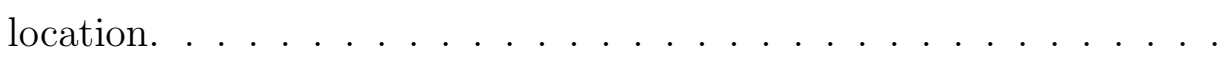


(a) Conical test cell \& lid. ................... 20

(b) $\mathrm{LH}_{2}$ in conical section. .................... 20

(c) Image normalized. . . . . . . . . . . . . . . . . 20

2.4 Experimental and numerical time response for the dry cell experiment with the $10 \mathrm{~mm}$ diameter $316 \mathrm{~L}$ SS test cell. . . . . . . . . 23

2.5 Stacked and averaged images with variation in pixel intensities along the highlighted row and column. ............... 27

2.6 Laplacian curve fit to the detected meniscus edge on stacked image of liquid hydrogen in Aluminum test cell. Symbols are the meniscus edge and the line is the Laplace curve representing the best fit to the edge data.

3.1 Neutron interaction with matter and cross sections . . . . . . 36

(a) Neutron interaction with matter............ 36

(b) Absorption and scattering cross sections .......... 36

3.2 Test setup showing the instrumented test cell attached to the end of a long SS sample stick which inserted into the sample well of the $70 \mathrm{~mm}$ "orange cryostat". ....................... 39

(a) $70 \mathrm{~mm}$ Cryostat ............................ 39

(b) Sample stick ............................ 39

(c) $10 \mathrm{~mm} \mathrm{Al} 6061$ test cell ...................... 39 
3.3 Time lapse images of liquid hydrogen phase change in a $10 \mathrm{~mm} \mathrm{Al} 6061$ test cell . . . . . . . . . . . . . . . . . .

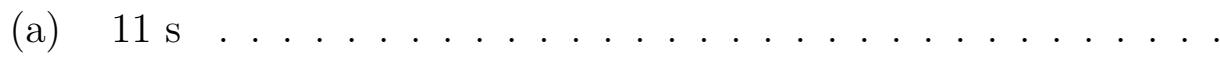

(b) $997 \mathrm{~s} \ldots \ldots \ldots \ldots \ldots$

(c) $1218 \mathrm{~s} \ldots \ldots \ldots \ldots \ldots$

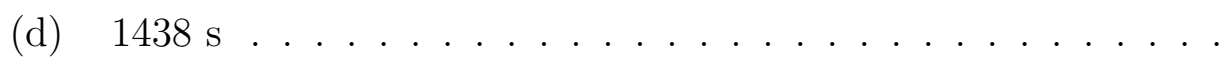

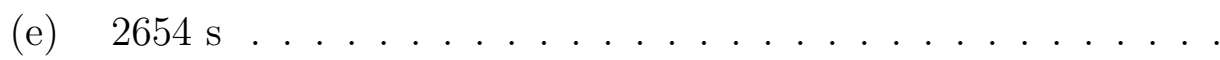

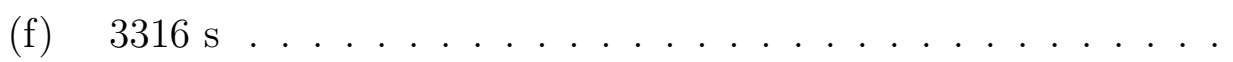

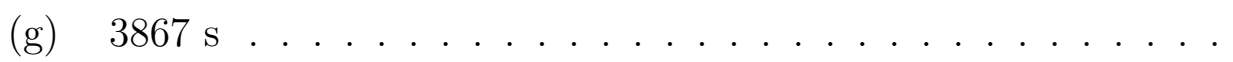

(h) $4475 \mathrm{~s}$

3.4 Determination of (a) rotation \& (b) liquid-wall interface and pixel pitch using pixel intensities shown by the red line and arrow. The image represents liquid hydrogen at $21 \mathrm{~K}$ in a $10 \mathrm{~mm}$ Al6061 test cell. . .

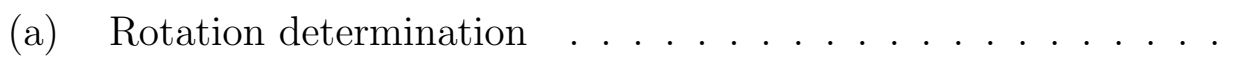

(b) Liquid-wall interface and pixel pitch determination . . . . . .

3.5 Histogram of pixel intensities for a typical liquid hydrogen in Al 6061 neutron image

3.6 Stacked image showing the three zones of analysis, horizontal and vertical intensities along the centerline . . . . . . . . . . . .

3.7 Edge detected and Young Laplace curves of liquid hydrogen in a 10 $\mathrm{mm} \mathrm{Al}$ cell at $19.9 \mathrm{~K}$ 
3.8 Sample optical density image of condensed liquid hydrogen in a $10 \mathrm{~mm}$ Al 6061 cell from the January 2015 test . . . . . . . . . . . . . .

3.9 Line profile of optical density along the liquid along with geometric liquid thickness (solid green line in figure 3.8) . . . . . . . . 57

3.10 Beam hardening fit to calculate attenuation coefficient . . . . . 57

3.11 Sample optical density image of condensed methane at $110 \mathrm{~K}$ and 117.2 $\mathrm{kPa}$, from the September 2015 test (a) and the corresponding line profile scan along the dashed red line ............. 59

(a) Sample optical density image .............. . . 59

(b) Line profile scan .................... 59

3.12 Phase change rates for hydrogen in the $10 \mathrm{~mm} \mathrm{Al}$ cell saturated at 21 $\mathrm{K}$. Condensation test is conducted at $19 \mathrm{~K}$ and evaporation at $23 \mathrm{~K}$

4.1 Hardware configuration for cryogenic phase change experiments. (a) Cryostat with test cell suspended in sample well. (b) Illustration of the 10-mm diameter test cell. s1, s2, s3, and s4 are the temperature sensors. The location of each (v1, v2, v3, v4) are relative to the bottom exterior surface of the test cell.

(a) Cryostat configuration ......................... 67

(b) 10-mm diameter test cell with sensor location ....... 67

4.2 Temperatures during thermal cycling of "dry" cell tests. "Heater" refers to the temperature sensor located in the cryostat heater block. 
(a) Hydrogen campaign, $10 \mathrm{~mm} \mathrm{SS} \mathrm{...} \mathrm{.} \mathrm{.} \mathrm{.} \mathrm{.} \mathrm{.} \mathrm{.} \mathrm{.} \mathrm{.} 70$

(b) Hydrogen campaign, $30 \mathrm{~mm} \mathrm{Al} \mathrm{\ldots ..........} 70$

(c) Hydrogen campaign, $10 \mathrm{~mm} \mathrm{Al} \mathrm{\ldots ..........} \mathrm{.} 70$

(d) Methane campaign, $10 \mathrm{~mm} \mathrm{Al} \mathrm{\ldots ..........} 70$

4.3 Temperature dependent thermal properties . . . . . . . . . 73

(a) Conductivity ....................... 73

(b) Specific heat $\ldots \ldots \ldots \ldots \ldots \ldots \ldots$

4.4 Computational domain of the thermal model . . . . . . . . . . 74

4.5 Comparison of simulation and hydrogen experiments for sensor s1. . 81

(a) Sensor s1 . . . . . . . . . . . . . . . . . . . . . . . . . . 81

(b) Sensor s1, log-temperature difference . . . . . . . . . . 81

4.6 Comparison of simulation and hydrogen experiments for sensor s2. . 82

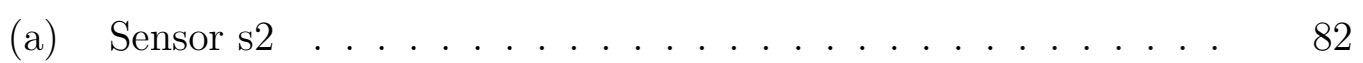

(b) Sensor s2, log-temperature difference . . . . . . . . . . . 82

4.7 Comparison of simulation and methane experiments for sensors s1 and s2.

(a) Sensors s1 and s2 . . . . . . . . . . . . . . . . . . 82

(b) Sensors s1 and s2, log-temperature difference . . . . . . . 82

4.8 Boundary conditions for vapor domain and liquid-vapor interface. . 86 
4.9 Temperature contour in the sample well and velocity streamlines in the vapor space of the test cell during steady state phase change of liquid hydrogen saturated at $21 \mathrm{~K}$. The heater was set at $23 \mathrm{~K}$. Streamlines emanate from the contact region and terminate at the exit of the test cell. Vapor velocities are less than $2 \mathrm{~cm} / \mathrm{s} . \ldots . . . . . . .$.

4.10 Temperature distributions along the exterior and interior of the test cell. The datum is located at the bottom of the test cell as shown in figure 4.1. Sensor s4 deviation due to $0.5 \mathrm{~K}$ bias error in the measurement. The contact line is located at $10 \mathrm{~mm} . \ldots \ldots$. . . . . .

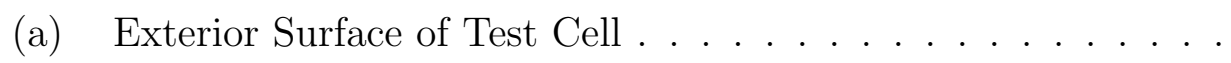

(b) Interior Surface of Test Cell . . . . . . . . . . . . .

5.1 Regions of an evaporating wetting meniscus . . . . . . . . .

5.2 Time lapse images captured during Run 1 using the $10 \mathrm{~mm} \mathrm{Al} \mathrm{cell} \mathrm{.}$

5.3 Film thickness measurements during Run 1 using the $10 \mathrm{~mm}$ SS 316 cell

5.4 Heat transfer paths between the heater and the test cell. . . . . . .

5.5 Contour of temperature results from the Macro-model for Run 2 using the $10 \mathrm{~mm} \mathrm{Al} \mathrm{cell.} \mathrm{The} \mathrm{result} \mathrm{shown} \mathrm{here} \mathrm{corresponds} \mathrm{to} \mathrm{the} \mathrm{final}$ converged value of $\alpha=0.58 \ldots \ldots \ldots \ldots$ 
5.6 Local interfacial temperature and mass flux along the liquid-vapor interface corresponding to results in figure 5.5. Zero value on the $\mathrm{x}$ axis refers to the apex of the meniscus. Results for film thickness $<10 \mu \mathrm{m}$ are neglected. . . . . . . . . . . . . . . . . .

(a) Temperature ....................... 110

(b) Mass flux .......................... 110

5.7 Solution of the transition film model for a $10 \mathrm{~mm} \mathrm{Al}$ cell containing hydrogen evaporating at $121.3 \mathrm{kPa}, \alpha=0.58$. . . . . . . . . .

5.8 Algorithm flowchart . . . . . . . . . . . . . . . . 121

5.9 Combined local mass flux values from the coupled multi-scale model for a $10 \mathrm{~mm} \mathrm{Al}$ cell containing hydrogen evaporating at $121.3 \mathrm{kPa}$,

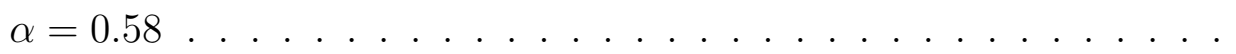

$5.10 \alpha$ vs $\dot{m}_{t f m}+\dot{m}_{m m}$ for a $10 \mathrm{~mm} \mathrm{Al}$ cell with hydrogen evaporating at $121.3 \mathrm{kPa}($ Run 1).

$5.11 \alpha$ vs Vapor Pressure for $\mathrm{LH}_{2}$. . . . . . . . . . . . . . . 124

A.1 Permission to reuse material in chapter $2 \ldots \ldots . . . . . .2165$

A.2 Permission to reuse material in chapter $3 \ldots \ldots \ldots 6$

A.3 Permission to reuse material in chapter $4 \ldots \ldots$. . . . . . 167

A.4 Permission to reuse material in chapter 7, page $134 \ldots . . . . .168$

A.5 Permission to reuse material in chapter 7, page 135 . . . . . . 169

A.6 Permission to reuse material in chapter $7,136 \ldots \ldots$. . . . . 170 
A.7 Permission to reuse material in chapter 7, page 137 


\section{List of Tables}

2.1 Test cells used during hydrogen experiments conducted in January 2015 at the NIST Neutron Imaging Facility. . . . . . . . . . . . . . .

3.1 Neutron cross sections and attenuation coefficients for $20 \mathrm{meV}$ neutrons<smiles></smiles>

[36]

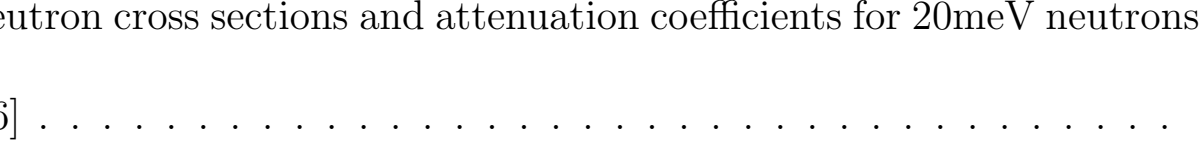

4.1 Test cells used in the hydrogen and methane experiments and the sensor locations as indicated in figure 4.2, All dimensions are in mm. .

4.2 Contact resistances determined from the hydrogen experiments. Units for contact area are $\mathrm{mm}^{2}$, conductivities are $\mathrm{W} / \mathrm{mK}$, and resistances are $\mathrm{K} / \mathrm{W} \ldots \ldots \ldots \ldots \ldots \ldots$

4.3 Contact resistances determined from the methane experiments with the $10 \mathrm{~mm} \mathrm{Al} \mathrm{cell.} \mathrm{Units} \mathrm{for} \mathrm{contact} \mathrm{area} \mathrm{are} \mathrm{mm}^{2}$, conductivities are $\mathrm{W} / \mathrm{mK}$, and resistances are $\mathrm{K} / \mathrm{W} \ldots \ldots . \ldots$

5.1 Summary of Cryo-Neutron tests conducted with $\mathrm{LH}_{2}$. . . . . . . .

5.2 The determined values of $\alpha$ for all test runs with hydrogen . . . . . 



\section{Preface}

My PhD research work was supervised by Dr. Jeffrey Allen and Dr. Chang Kyoung Choi at Michigan Technological University from 2014 to 2018. The work in this dissertation has been published in 7 peer reviewed articles and at least one more article will be submitted in the near future. Out of the 7 published articles, 3 are full length peer reviewed journal publications and 4 are peer reviewed photogallery entries in the Journal of Heat transfer.

Chapter 2 contains material previously published in Cryogenics by Bellur et al. [19]. I was part of the team that conducted cryo-neutron imaging experiments at the National Institute of Standards and Technology (NIST) in January 2015. The experiments were a group effort that included my advisors Dr. Jeffrey Allen and Dr. Chang Kyoung Choi, Dr. Ezequiel Medici and myself from MTU along with Dr. Daniel Hussey, Dr David Jacobson, Dr. Jacob LaManna and Dr. Juscelino B. Leao from NIST. In addition to experiment preparation and setup, my contribution also included setting up the data/image acquisition, data analysis, post-processing and interpretation of test data. Thermal model described in section 3 of the manuscript was primarily the work of Arun Tamilarasan and Dr. James Hermanson from University of Washington. However, I collaborated with Arun remotely, provided constant feedback and helped develop the boundary conditions for the results published. Section 4 
was a collaborative effort between myself, Manan Kulshreshta and Dr. Vinaykumar Konduru. The manuscript text was a group effort written primarily by myself, Dr. Jeffrey Allen, Dr. Ezequiel Medici and Dr. Vinaykumar Konduru.

Chapter 3 contains material previously published in Journal of Flow Visualization and Image Processing by Bellur et al. [23]. In addition to the January 2015 experiments, this work includes data from the July 2015 and September 2015 of which I was an integral part. I'm responsible all aspects of the data analysis and image processing presented in the manuscript. Manan Kulshreshta and I developed the interface tracking method under the supervision of Dr. Vinaykumar Konduru and while I developed the optical density method with the help of Dr. Daniel Hussey. I'm the primary author of all text, figures and tables in the manuscript.

Chapter 4 contains material previously published in Cryogenics by Bellur et al. [20]. I built the thermal model published in this manuscript from scratch. Although the geometry of the model is similar, the approach used is different from that used by Arun Tamilarasan in Bellur et al. [19] and Chapter 2 of this dissertation. I'm the primary author of all text, figures and tables in the manuscript.

Chapter 7 contains material previously published in Journal of Heat Transfer by Bellur et al. [21, 22, 24], Konduru et al. [77]. These are short, rapid publication photogallery entries that focus on various unique results obtained from neutron imaging. I'm the primary author of all text and figures in Bellur et al. [21, 22, 24] while 
Dr. Vinaykumar Konduru is the primarily responsible for the analysis that resulted in Konduru et al. 77] while I helped with some aspects of image processing and manuscript generation. 



\section{Acknowledgments}

First and foremost, I would like to thank both my advisors Dr. Jeffrey Allen and

Dr. Chang Kyoung Choi for their constant support, guidance and willingness to impart knowledge. This undertaking could not have been possible without them. Dr. James Hermanson from University of Washington was very helpful in providing feedback on various aspects of the modeling effort. I acknowledge Dr. Daniel Hussey, Dr. David Jacobson, Dr. Jacob LaManna and Dr. Juscelino B. Leao's contributions to the experiments conducted at NIST. I would like to extend my thanks to Dr. Ezequiel Medici, Dr. Kazuya Tajiri, Dr. Gowtham, Dr. Gregory Odegard and Dr. Vinaykumar Konduru for helping me every step of the way being both a mentor and a friend, as and when needed. Their support, feedback, criticism and friendship has been invaluable both academically and personally. The MnIT and $\mu$-EOS research groups have always been a source of insightful discussion and camaraderie.

I thank my parents for their unconditional love and encouragement to follow my dreams. I have a special appreciation for Rick and Michael Stanitis, my Canterbury House and Tools of Ignorance families, and all the wonderful people I have gotten to know because of them. I extend my gratitude to my friends Ameya, Neel, Bethany, Anuj, Pratik, Chethan, Falak, Tara and countless others. In short, I'd like to thank everyone I've raised a pint with or thrown a softball at. 
I'm indebted to Nupur Bihari, who has been by my side through thick and thin, even when we ran out of gas, multiple times. Your presence was always felt as well.

Finally, I'm grateful for the various sources of support throughout my time at MTU:

(1) NASA's Space Technology Research Grants Program (grant \# NNX14AB05G)

(2) Department of Mechanical Engineering - Engineering mechanics, for the Winnikow fellowship and multiple graduate assistantships (3) Graduate School, for the doctoral finishing fellowship, (4) Graduate Student Government, Cryogenics Society of America and American Society of Space and Gravitational Sciences for travel awards. 


\section{Abstract}

The control of propellant boil-off is essential in long-term space missions. However, a clear understanding of cryogenic propellant phase change and the values of accommodation coefficients are lacking. To that effect, a new method to determine accommodation coefficients using a combination of neutron imaging, thin film evaporation modeling and CFD modeling has been established. Phase change experiments were conducted in the BT-2 Neutron Imaging Facility at the National Institute of Standards and Technology (NIST) by introducing cryogenic vapor (H2 and $\mathrm{CH} 4$ ) at a set pressure into Al6061 and SS316L test cells placed inside a 70mm cryostat. Condensation is achieved by lowering the cryostat temperature below the saturation condition and vice versa for evaporation. Neutron imaging is used to visualize the liquid-vapor interface inside metallic containers due to the difference in attenuation between the cryogen and the metal. Phase change tests are conducted using liquid hydrogen and methane at a range of saturation points between $80-230 \mathrm{kPa}$ and corresponding phase change rates were determined. The contact resistances and other transient heat transfer properties of the cryostat setup is determined from the combination of a CFD thermal transport model and a dry thermal cycling test. The calibrated CFD model then allows for the determination of the inner wall temperature profile. Results from neutron imaging and the thermal model serve as boundary conditions to a multiscale evaporation model. A macroscale 2D FEA model is used to 
compute evaporation flux in the bulk meniscus while a thin film evaporation model is used to account for enhanced evaporation near the contact line. Using a combination of neutron imaging, CFD thermal model and a multiscale evaporation model, there is a possibility to extract the accommodation coefficient while accounting for the curvature, disjoining pressure and a variable interface temperature. The accommodation coefficient of $\mathrm{H} 2$ decreases from $0.65 \pm 0.12$ at $88 \mathrm{kPa}$ to $0.22 \pm 0.1$ at 226 $\mathrm{kPa}$ and is independent of container material/geometry. The error is dominated by the uncertainty in the temperature measurements $(0.25 \mathrm{~K})$. 


\section{Chapter 1}

\section{Introduction}

Phase change is ubiquitous and kinetic theory is shown to be very effective in describing liquid-vapor phase change. However, the ability to predict the rate of phase change inside propellant tanks remains a challenge primarily due to the absence of evaporation/condensation coefficients, also called accommodation coefficients. These coefficients are inputs to the kinetic theory equations and are necessary to develop reliable models for predicting boil-off and cryo-storage stability for liquid propellants in low gravity. For water alone, decades of research has yielded accommodation coefficients that span several orders of magnitude. The current state of knowledge on evaporation/condensation processes is insufficient for designing large cryogenic depots critical to long-term space exploration missions. There is currently no experimental 
data available on controlled evaporation/condensation rates or corresponding accommodation coefficients of cryogenic propellants.

The objective of this study is to develop a combined experimental and computational technique to determine accommodation coefficients of cryogenic propellants. Neutron imaging was employed as a visualization tool to measure and characterize evaporation/condensation rates of liquid hydrogen and methane inside Al 6061 and SS 316 test cells of various sizes and shapes. Tests were conducted at various saturation conditions and a variety of phase change rates were obtained through accurate pressure and temperature control. During the experiments, temperature measurements could only be made at discrete points on the outer wall of the test cells. In order to characterize the thermal transport and estimate an inner wall temperature distribution from discrete outer wall temperature measurements a thermal transport model was built. Finally, a multi-scale evaporation model was built such that the imaging results provide the physical boundary conditions and the phase change rates while a thermal model provides the temperature boundary conditions. Using a combination of neutron imaging results, thermal modeling and multi-scale evaporation modeling, a unique value of the accommodation coefficient could be obtained. The variation in the measured coefficient with size of the test cell, vapor pressure, surface chemistry is described.

Chapter 2 contains material previously published in Cryogenics by Bellur et al. [19]. 
It focuses on the experimental setup including temperature-pressure control, cryostat operation and details preliminary results of neutron imaging.

Chapter 3 contains material previously published in Journal of Flow Visualization and Image Processing by Bellur et al. [23]. It focuses on the neutron imaging setup and post-processing of captured neutron images.

Chapter 4 contains material previously published in Cryogenics by Bellur et al. [20]. It focuses on the thermal transport model aimed at determining the inner wall, solidfluid interface temperature distribution from discrete outer wall temperature measurements.

Chapter 5 details the multi-scale evaporation model and the methodology used to determine the accommodation coefficients for hydrogen. It is formatted as a draft of a manuscript to be submitted to a high impact journal shortly after the completion of the degree.

Chapter 6 contains an overview of the entire research effort and outlines possible avenues for fundamental investigations on evaporation/condensation processes in addition to phase change with cryogens.

Chapter 7 contains material previously published in Journal of Heat Transfer by 
Bellur et al. [21, 22, 24], Konduru et al. [77]. These are short, rapid publication photogallery entries that focus on various unique results obtained from neutron imaging.

In summary, this dissertation is a compilation of 7 previously published articles and 1 article that will be submitted in the near future. Out of the 7 published articles, there are 3 full length peer reviewed journal publications and 4 peer reviewed photogallery entries in the Journal of Heat transfer. 


\section{Chapter 2}

\section{A new experiment for investigating}

evaporation and condensation of

\section{cryogenic propellants}

Passive and active thermal and fluid control systems are routinely used to manipulate cryogenic liquids in microgravity and to mitigate boil off. Computational fluid dynamics (CFD) modeling of the propellant coupled with a lumped thermodynamic treatment of the vapor phase has been used to study pressurization within cryogen tanks [13, 99 102]. From these models, a thin (approximately $1 \mathrm{~mm}$ ) liquid layer separating the vapor phase from the wall was shown to form. Accurately predicting the

The material contained in this chapter was previously published in Cryogenics by Bellur et al. [19]. See Appendix A for documentation of permission to republish this material. 
stability of the liquid layer and evaporation/condensation remains a challenge due to the absence of reliable values of evaporation and condensation coefficients and the ability to computationally capture the local thermodynamics [60, 99, 101].

Additional experimental evidence that suggests understanding local thermodynamic states is critical to predicting phase change of liquid hydrogen and methane were found during recent tests conducted at National Aeronautics and Space Administration (NASA) Glenn Research Center located in Cleveland, Ohio. These experiments focused on determination of bubble-point pressure, i.e. vapor break through, for liquid oxygen, methane, and hydrogen in liquid acquisition screens. The uncertainty in the experimental data was largely attributed to uncertainty in the evaporation at the screen surface $[60$ 62]. Meniscus phase change is significantly different than that of pool boiling, where vapor pressure and wall temperature are generally sufficient to predict heat flux. Two additional factors affect phase change at a meniscus, such as those present in a screen. The first is curvature, which gives rise to a pressure jump across the liquid-vapor interface due to surface tension. The second factor is the presence of the contact line, which results in non-uniform evaporation over the liquid surface due to anisotropy in the liquid stresses within the thin liquid film due to disjoining pressure effects [106]. Curvature and disjoining pressure effects have been incorporated into evaporation and condensation models [1, 106, 110, 124], but accurate measures of the evaporation and condensation coefficients remains a challenge, especially for cryogenic liquids. 
Liquid-vapor (evaporation or condensation) phase change is a complex, multiscale, conjugate problem. Different phase change models have been proposed to quantify the rate of mass transfer form one state to the other. These models can be classified as diffusive or kinetic models. Diffusive models rely upon relative partial pressure for triggering phase change. Diffusive evaporation or condensation models are material independent, generating the same mass flux regardless of the contact angle or liquid phase curvature. These models have been applied to study phase change in liquid reservoirs with large open surface area. In contrast, models traditionally used for investigating phase change in smaller liquid reservoirs where the exposed surface is comparable to the meniscus size, such as in porous media, are kinetics based. Kinetic models are dependent upon the material properties, the location of liquid phase within the material and may take into account the effect of disjoining pressure, meniscus curvature, and non-equilibrium interface temperature [98].

When evaporation is diffusion limited, the rate of phase change is proportional to the interfacial area and to the concentration difference between the vapor and the liquidvapor interface, which for the modeling purposes is considered to be saturated vapor. A change in substrate material has no effect on the rate of phase change. As the interfacial area decreases with respect to contact line length, the rate of evaporation is no longer proportional to the area and a different model is needed to predict the mass flux based on the molecular dynamics taking place at the contact line region. 
The contact line is an apparent intersection of three phases - vapor, liquid and solid. This intersection is commonly described using the static contact angle, $\theta$, which refers to the apparent angle between the liquid and the solid (as measured through the liquid). The contact line is a continuum region and is often described for wetting liquids as a continuously thinning film that terminates in an absorbed layer. Figure 2.1 delineates regions of interest along a wetting evaporating meniscus according to the component of normal stress most affecting the thermo-fluid dynamics. The normal stress in the bulk is mostly affected by capillarity, or interface curvature. The adsorbed film region is characterized by intermolecular forces and is not optically accessible. Both intermolecular forces and curvature affect the normal stress in the contact line region. A typical value of the maximum thickness of the contact line region where intermolecular forces begin to affect the liquid interface shape is 1 micrometer.

The contact line region has a dramatic effect on evaporation (and condensation). Typically during phase-change a relatively large temperature gradients is setup in the contact line region both parallel and perpendicular to the solid surface that may result in thermocapillary stresses. For non-polar and/or wetting liquids, the result is that $60 \%$ to $90 \%$ of the total evaporation occurs in the contact line region [43, 47, 49, 53, 55, 65, 93, 97, 106, 108, 109, 114, 115, 121, 126, 127]. Though specific experiments have been empirically analyzed and numerical models validated against these experiments [43, 106, 115], the effect of contact angle and contact line length on total evaporation is not quantitatively predictable in a general sense. 


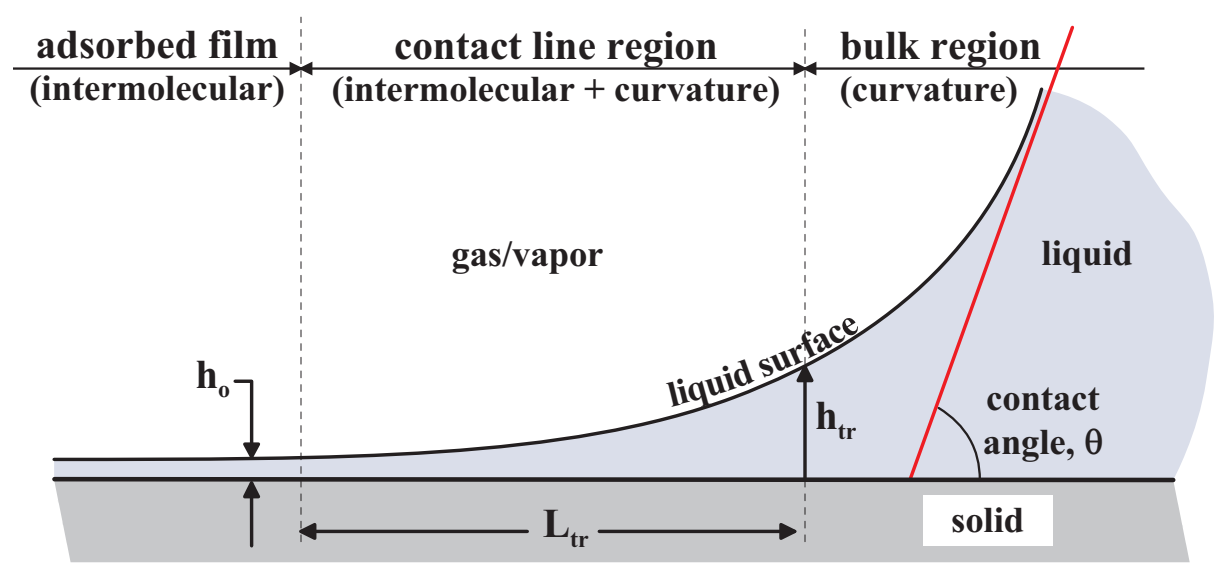

Figure 2.1: Extended meniscus with regions delineated by normal stress components.

A kinetic model depends upon the local interface conditions in the contact line region, specifically in the contact line region. The amount of mass undergoing phase change is proportional to the size of the contact line region as well as the local properties such as partial pressure of vapor, temperature, and relative humidity. The mass undergoing phase change can be expressed in the form of the Hertz-Knudsen-Schrage equation [1, 106, 116]. Originally developed from kinetic theory for planar evaporation, this model has been expanded to include the effects surface tension [110] and surface curvature [124] through the use of the Clapeyron equation:

$$
J=\frac{2 \alpha}{2-\alpha}\left(\frac{M}{2 \pi R T_{\mathrm{lv}}}\right)^{1 / 2}\left[\frac{p_{\mathrm{v}} M h_{\mathrm{fg}}}{R T_{\mathrm{v}} T_{\mathrm{lv}}}\left(T_{\mathrm{lv}}-T_{\mathrm{v}}\right)-\frac{v_{\mathrm{l}} p_{\mathrm{v}}}{R T_{\mathrm{lv}}}(\Pi+\sigma \kappa)+\frac{M g p_{\mathrm{v}}}{R T_{\mathrm{v}}} x\right]
$$

where $J$ is the evaporative flux, $\alpha$ is the is the evaporation or condensation coefficient (often referred to as the accommodation coefficient), $T_{\mathrm{lv}}$ is interfacial temperature, $\Pi$ is the disjoining pressure (the net pressure reduction within the film due to the 
solid-liquid intermolecular forces), $\sigma$ is surface tension, and $\kappa$ is the surface curvature. Other parameters are standard thermodynamic properties [124].

Evaporation and condensation coefficients, often referred to as accommodation coefficient, are derived from kinetic theory and represent the fraction of molecules striking the liquid surface [1]. The accommodation coefficient is considered to be a thermodynamic property of kinetic models of evaporation and condensation. Accurate prediction of the rate of phase change typically requires a measured value of the accommodation coefficient.

Unfortunately, there is significant discrepancy in reported values of the accommodation coefficient. For water alone the values have varied by two to three orders of magnitude depending on the researcher or the method used to determine this coefficient. An indication of why there is such a large discrepancy in the mass accommodation coefficient can be inferred from experiment details described by Cammenga et al. [33] and reiterated in Marek and Straub [89]. An evaporation coefficient of 0.002 was found for water in a glass vessel, but when the glass vessel was replaced with a copper vessel the evaporation coefficients increased two orders of magnitude to values between 0.25 and 0.38 . With the exception of the vessel wall material, both experiments were conducted in the same apparatus. Thus, the reported values of the accommodation coefficient do not reflect the local conditions nor the non-uniform evaporation that occurs due to the presence of a contact line. 
To solve for the mass flux undergoing phase change the temperature in the liquid phase is required. The liquid temperature depends on the adjacent solid surface temperature. Liquid and solid temperature profiles can be obtained from a conjugate heat transfer model that incorporates representative boundary conditions of the system under observation. For this research, a computational thermal model of the test cell and sample well developed in ANSYS/Fluent is used to obtain the temperature profile on the interior solid surface of the test cell. The liquid temperature profile is obtained through integration of a lubrication model [127] using the computational results for the thermal boundary conditions. The total mass evaporated, and subsequent heat transfer, is found by integrating equation (2.1) from the absorbed film region to the bulk meniscus region.

To that end, a new type of experiment with complimentary computational analysis has been undertaken to determining the evaporation and condensation coefficients for liquid hydrogen and methane. A combined modeling and experimental effort is being pursued with the experiments conducted at the Neutron Imaging Facility (NIF) located at the National Institute of Standards and Technology (NIST) located in Gaithersburg, Maryland. Results from evaporation and condensation experiments will be discussed in forthcoming manuscripts. The focus of this manuscript will be restricted to the use of the dry cell tests for calibration of a CFD thermal model and edge detection of liquid hydrogen surfaces inside the aluminum and stainless steel test cells. 


\subsection{Neutron Imaging Experiments}

Experiments examining the bulk evaporation and condensation of liquid hydrogen were conducted during January 2015 at the NIST Center for Neutron Research (NCNR) in the Neutron Imaging Facility. Thermal neutrons $(E \approx 25 \mathrm{meV})$ neutrons from a fission reactor penetrate a cryostat that contains a steel or aluminum test apparatus. The large neutron scattering cross section of hydrogen as compared to that of steel and aluminum allows for signal-to-noise levels sufficient for imaging the location of the liquid hydrogen surface within the test cells.

The scintillator used for imaging is a $7.6 \mathrm{mg} / \mathrm{cm}^{2}$ Gadoxysulfide screen with a thickness of $20 \mu \mathrm{m}$. An Andor NEO sCMOS (scientific Complementary Metal Oxide Semiconductor) camera with a pixel pitch of $6.5 \mu \mathrm{m}$ and variable exposure time is used to capture the images. An $85 \mathrm{~mm}$ Nikon lens with a PK13 extension tube was used to image the scintillator light. This detector configuration has sufficient spatial $(<50 \mu \mathrm{m})$ and temporal $(<10 \mathrm{~s})$ resolution to measure local curvature and evaporation rates of liquid hydrogen. Additional details on the Neutron Imaging Facility (NIF) and the hydrogen infrastructure used for the experiments described herein can be found in Hussey et al. [68, 69].

A schematic of the cryostat is shown in figure 2.2. The sample well passes through 
concentric vacuum and cryogen annuli and then extends below these annuli into an evacuated chamber through which the neutron beam passes. The outer most cryogen annulus is filled with liquid nitrogen that evaporates and vents to the atmosphere thereby maintaining a temperature of $77 \mathrm{~K}$. An inner liquid helium jacket also evaporates. The rate of helium evaporation and therefore the rate of cooling and minimum temperature is controlled through a throttling valve that can be adjusted. For additional cooling a vacuum can be pulled on the vapor side of the throttling valve. The helium throttle valve is part of an assembly referred to as the copper block that is positioned at the separation between the cryostat and the lower chamber through which the neutron beam passes. An electric heater is also located in this copper block. In this manner, the temperature of the copper block can be set and controlled. Thermal energy is transferred to and from the test cell (suspended in the bottom of the sample well) by a combination of (i) conduction from the copper block through an aluminum radiation baffle and down a stainless tube to the test cell, and (ii) convection in low pressure helium gas circulating between the test cell and the sample well housing. The stainless tube with radiation baffles to which the test cell is attached is referred to as the sample stick.

The cryostat is prepared by filling with liquid nitrogen and liquid helium to begin the cool down. The sample well is filled with helium gas at approximately $135 \mathrm{kPa}$ absolute (19.6 psia). The helium is allowed to continuously flow into and out of the sample well so as to prevent the introduction of air or water vapor. While the helium 
gas is flowing through the sample well, the sample stick with the test cell assembly is inserted and secured with an o-ring seal in the top flange. Then the helium gas is completely evacuated from the sample well to a pressure of $10^{-6}$ torr and reintroduced at $125 \mathrm{kPa}$ to $135 \mathrm{kPa}$ absolute. This evacuation and refilling procedure is performed three times in order to thoroughly purge the sample well of any residual air and water vapor that might form an ice blockage and prevent free movement of the sample stick. Following the last evacuation of the sample well, a small quantity of helium gas is reintroduced with a pressure between $10 \mathrm{~Pa}$ and $200 \mathrm{~Pa}$ absolute. At the time of these experiments, the exact pressure of the sample well during testing was not available. Even with this pressure known, however, the density of helium surrounding the test cell would be difficult to determine due to the temperature gradient that exists along the sample well $(\approx 20 \mathrm{~K})$ from the test cell up to the flange seal $(\approx 300 \mathrm{~K})$. Since the density of the helium in the sample well is not known, a sequence of complimentary experiments and numerical simulations are performed in order to characterize the conductive and convective heat transfer from the cryostat copper block to the test cell. This procedure is discussed later.

A hydrogen generator with a control manifold is used for these tests. For the hydrogen evaporation-condensation experiments, the manifold is configured to allow for three functions. The first is to be able to pull a hard vacuum on the entire manifold and the test cell, which is connected to the manifold via a $1 / 8$ inch diameter stainless steel line. In this manner, the test cell can be evacuated and a leak check performed 


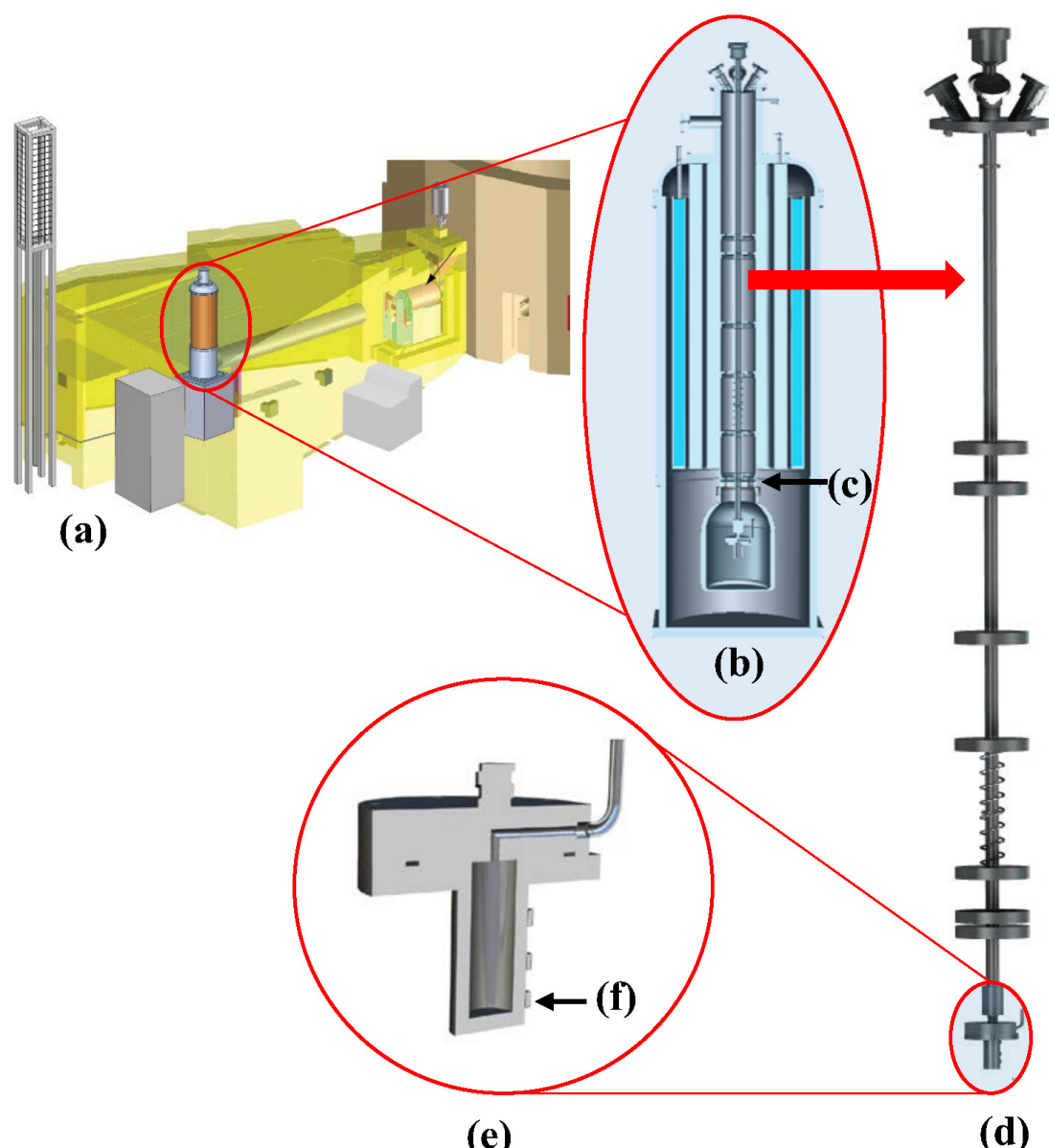

Figure 2.2: $\quad$ Overview of experiments conducted at the NIST Neutron Imaging Facility (NIF). (a) Neutron Imaging Facility with cryostat in beam line. (b) Cryostat with test cell installed. (c) Location of copper block used for heating and cooling the test cell and helium gas in the sample well. (d) Sample holder with $10-\mathrm{mm}$ test cell attached. (e) Cutaway view of the 10 mm diameter test cell and lid.

prior to introduction of the hydrogen. The second function of the manifold is to purge the manifold and the test cell with gaseous helium for pressurized leak checks. Finally, the third function of the manifold is to introduce hydrogen to the test cell and to accurately control the vapor pressure. For evaporation tests, the vapor pressure established at the manifold is set slightly below the saturation pressure at the temperature set point of the cryostat. Alternatively, the set point of the cryostat can 
be set above the saturation temperature of the pressure set point of the manifold. For condensation, these conditions are reversed.

Once the leak checks were satisfactorily completed, hydrogen gas is delivered to the manifold at $1380 \mathrm{kPa}$ (200 psig) from the generator. The pressure of the hydrogen gas is decreased using a regulator to between $100 \mathrm{kPa}$ and $200 \mathrm{kPa}$ absolute (15 psia to 30 psia). The hydrogen flows continuously through the manifold to the atmospheric vent. The pressure in the gas manifold is adjusted to the desired saturation pressure $\pm 100 \mathrm{~Pa}( \pm 1 \mathrm{mbar})$ by throttling the pressure from the manifold to the vent using a needle valve. With this arrangement, hydrogen could be transferred to or from the test cell during condensation or evaporation, respectively, without changing the pressure set point. When testing at hydrogen saturation pressures close to atmospheric pressure, the manifold is vented through a vacuum pump prior to exiting at the atmospheric vent. In this way, there remains sufficient pressure differential across the throttling valve for accurate pressure control.

Four test cell configurations were used during testing. The configurations were varied in order to investigate the effect of (i) surface properties that affect the disjoining pressure term in equation 2.1, (ii) geometric properties that affect the curvature term, and (iii) contact line length to liquid-vapor surface area. Additional design constraints include volume restrictions for safety considerations and diameter constraints for imaging considerations. The latter required that there be sufficient liquid-vapor 
interface curvature so that there was never the possibility of interface-pixel alignment; that is, the liquid-vapor interface never aligns with a single row of pixels. This condition would result in a large uncertainty in the interface location since the pixel dimensions are 25 micrometers. In addition, the diameter of the test cell needed to be large enough that there were sufficient pixels across the interface for accurate edge detection. This latter condition was met by maintaining a Bond number between 1 and 100. The Bond number is defined as $\Delta \rho g r^{2} / \sigma$, where $\Delta \rho$ is the density difference across the liquid-vapor interface, $g$ is gravitational acceleration, $r$ is the radius of the test cell, and $\sigma$ is surface tension. Table 2.1 lists the pertinent details of each test cell.

The first test cell is a $5 \mathrm{~mm}$ diameter cylinder connected to a $30 \mathrm{~mm}$ diameter cylinder through a conical section with a 10 degree slope from the horizontal. Figure 2.3(a) illustrates this test cell. The remaining three of the test cells are cylindrical in shape. One purpose of the conical test cell was to investigate the possible existence of a finite contact angle with hydrogen on aluminum, which was suggested from preliminary experiments conducted in September 2014. The preliminary experiments suggested a contact angle as large as 10 degrees. If this condition were to exist, then the liquidvapor interface should be perfectly flat in the 10 degree conical transition regardless of the Bond number. From figure 2.3(a), the interface is indeed flat in this region. While suggestive of a finite contact angle, a more quantitative analysis of interface curvature and the possibility of a finite contact angle is discussed later. All test cells 
Table 2.1

Test cells used during hydrogen experiments conducted in January 2015 at the NIST Neutron Imaging Facility.

\begin{tabular}{|c|c|c|c|c|c|}
\hline $\begin{array}{l}\text { Test } \\
\text { Cell }\end{array}$ & Material & Shape & $\begin{array}{l}\text { Inner } \\
\text { Diameter }\end{array}$ & $\begin{array}{c}\text { Wall } \\
\text { Thickness }\end{array}$ & $\begin{array}{c}\text { Bond } \\
\text { number }\end{array}$ \\
\hline 1 & $6061 \mathrm{Al}$ & conical & $\begin{array}{l}5 \mathrm{~mm} \& 30 \mathrm{~mm} \text {, } \\
10^{\circ} \text { transition }\end{array}$ & $3 \mathrm{~mm}$ & $2.3,84.5$ \\
\hline 2 & 316L SS & cylindrical & $10 \mathrm{~mm}$ & $3 \mathrm{~mm}$ & 9.3 \\
\hline 3 & $6061 \mathrm{Al}$ & cylindrical & $30 \mathrm{~mm}$ & $3 \mathrm{~mm}$ & 84.5 \\
\hline 4 & $6061 \mathrm{Al}$ & cylindrical & $10 \mathrm{~mm}$ & $3 \mathrm{~mm}$ & 9.3 \\
\hline
\end{tabular}

used a common lid fabricated from 316L stainless steel. The test cell lid includes a vapor passage that is also shown in figure 2.3(a).

The four test cells and stainless steel lid were cleaned using an acid solution, which is made from sulfuric acid $\left(\mathrm{H}_{2} \mathrm{SO}_{4}, 96 \%\right.$ concentration $)$ and hydrogen peroxide $\left(\mathrm{H}_{2} \mathrm{O}_{2}\right.$, $35 \%$ concentration) in 3:1 volumetric ratio, respectively. The parts to be cleaned were immersed in the acid solution for 10 to 20 seconds after which it was immediately immersed in a beaker with $90^{\circ} \mathrm{C}$ distilled water. The part was then immersed in another beaker with distilled water at room temperature. After rinsing with distilled water, the components are blown clear of any residual water using dry nitrogen, allowed to cool and sealed in plastic bags. For the aluminum test cells, this procedure results in an oxide formation and an increase in the surface roughness. The mean surface roughness is between 1 micrometer and 10 micrometers.

Instrumentation for these experiments consisted of pressure measurements on the test cell feed line and manifold as well as temperature measurements on the test cell exterior, the helium in the sample well, and the cryostat. Three Lakeshore silicon 
diode DT-670 temperature sensors were mounted on the outside of the test cell and secured in place by use of custom fabricated 306 stainless steel springs that wrapped around the test cell exterior. A fourth sensor was suspended in the sample well near the test cell to measure the temperature of the circulating helium gas. Test cell temperatures were logged using a Lakeshore Model 340. Calibration curves incorporated into the Lakeshore Model 340 for this series of sensor were used for converting the sensor signals to temperatures. The uncertainty in the test cell temperature measurements is $\pm 0.25 \mathrm{~K}$. The uncertainty in the cryostat heater temperature is $\pm 0.1 \mathrm{~K}$. The temperature of the copper block (NTC RTD X45720 sensor) and the sample holder temperature (Scientific Instruments Si410B sensor) were logged using a Lakeshore Model 331 that was also used to control the heater temperature. Pressures were logged using two Mensor pressure transducers. One sensor (Mensor CPG 2500) was connected to the hydrogen gas feed connected to the test cell. The second pressure transducer (Mensor DPG 15000) was connected to the manifold. The uncertainty in the pressure measurements is $0.01 \%$ of the reading.

For each test cell, three types of experiments were conducted - dry cell, condensation, and evaporation. The 'dry cell' tests were conducted to provide transient thermal response data to be used later in calibrating the computational thermal model. A vacuum of $10^{-6}$ torr was pulled on the manifold which was open to the test cell. While under continuous vacuum, the temperature of the test cell was increased and decreased by adjusting the cryostat set point. 


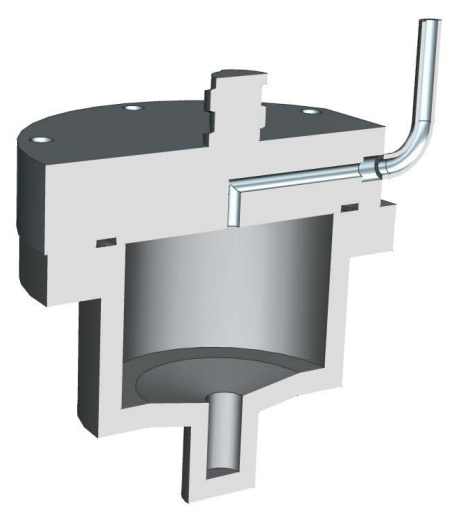

(a) Conical test cell \& lid.

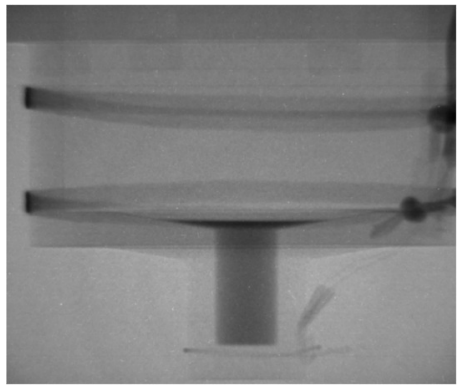

(b) $\mathrm{LH}_{2}$ in conical section.

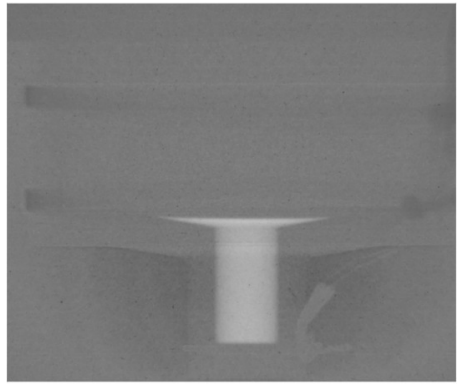

(c) Image normalized.

Figure 2.3: (a) Cutaway view of conical test cell. The smaller cylinder is $5 \mathrm{~mm}$ diameter and the larger cylinder is $30 \mathrm{~mm}$ diameter. The conical section has a 10 degree slope from the horizontal. (b) Liquid hydrogen in an aluminum 6061 test cell. A silicon diode sensor attached to the $5 \mathrm{~mm}$ diameter section is faintly visible. The flat hydrogen interface in the conical portion indicates the presence of a contact angle of approximately 10 degrees.

(c) Normalized image emphasizing liquid hydrogen location.

\subsection{Thermal Modeling of Test Cells}

The design of the experiment is such that no temperature measurements can be made on the inside of the test cell and no transient heat transfer data is available for the cryostat. In order to extract the evaporation and condensation coefficients, the evaporation model (equation 2.1) requires a thermal boundary condition on the interior wall of the test cell. In order to determine the appropriate interior wall temperature distribution, an axisymmetric computational thermal model was developed using AN-

SYS/Fluent. Coupling of pressure and velocity is achieved using the SIMPLEC (Semi 
Implicit Method for Pressure Linked Equations Coupled) algorithm and the convergence criteria were set to $10^{-6}$ for all residuals of momentum, energy and continuity equation. Temperature dependent material properties of the test cell and cryostat were employed to improve solution accuracy.

The ANSYS/Fluent thermal model includes the test cell and lid, the sample stick up to the first radiation baffle, the cryostat copper block and the sample well enclosure, which is an aluminum canister secured to the copper block. These components are illustrated in figure 2.2. Heat is transferred to and from the test cell by a combination of heat conduction through the radiation baffle and sample stick as well as through convection in the helium gas surrounding the test cell and contained within the sample well enclosure. The amount of helium in the sample well surrounding the test cell could not be precisely measured and the amount of helium changes when changing out the test cell being tested. The pressure in the sample well is estimated to be between $10 \mathrm{~Pa}$ and $200 \mathrm{~Pa}$. An added complexity arises from the the helium surrounding the test cell interacting with helium in the sample well above the bottom radiation baffle, which supports a temperature gradient increasing from the bottom to the top of the cryostat. As the test cell is cooled and heated, there is net movement of helium gas between the sample well enclosure and the sample well volume above the bottom radiation baffle.

The thermal model is tuned using the transient response of the test cell and the sample 
well helium gas during the dry cell thermal cycling tests. The sample well enclosure, which is fabricated from aluminum 6061, is initially set to the same temperature as the copper block and the test cell. The transient temperature of the copper block is varied in the model to match the experimental results. The numerical temperatures that correspond in location to the experimental sensors, three on the test cell and one in the helium gas, are determined from the simulation and compared to the experimental values. Matching the transient response for all four sensors requires minor adjustments to the thermal diffusivity of the helium gas and contact resistance between the copper block and the bottom radiation baffle. This procedure is repeated for each test cell. Once the thermal model results match the transient sensor data, then the effective heat transfer conditions, namely contact resistances and helium gas density, for each test cell are established. These conditions are presumed to remain constant during the subsequent condensation and evaporation experiments. Figure 2.4 illustrates a 'matched' transient response for one of the silicon diode sensors on the 10-mm-diameter 316L SS test cell.

After the heat transfer conditions were established for the 10-mm-diameter aluminum test cell, a grid sensitivity study for the thermal model was conducted using 20000, 30000,46000 , and 65000 nodes. After 350 seconds of simulation, the deviation of the numerically predicted temperature from the measured temperature at the bottom of the test cell varied between $0.9 \%$ at the two highest node counts, 1.2 percent at 30000 nodes, and $2.15 \%$ at 20000 nodes. A node count of 30000 was selected as the optimum 


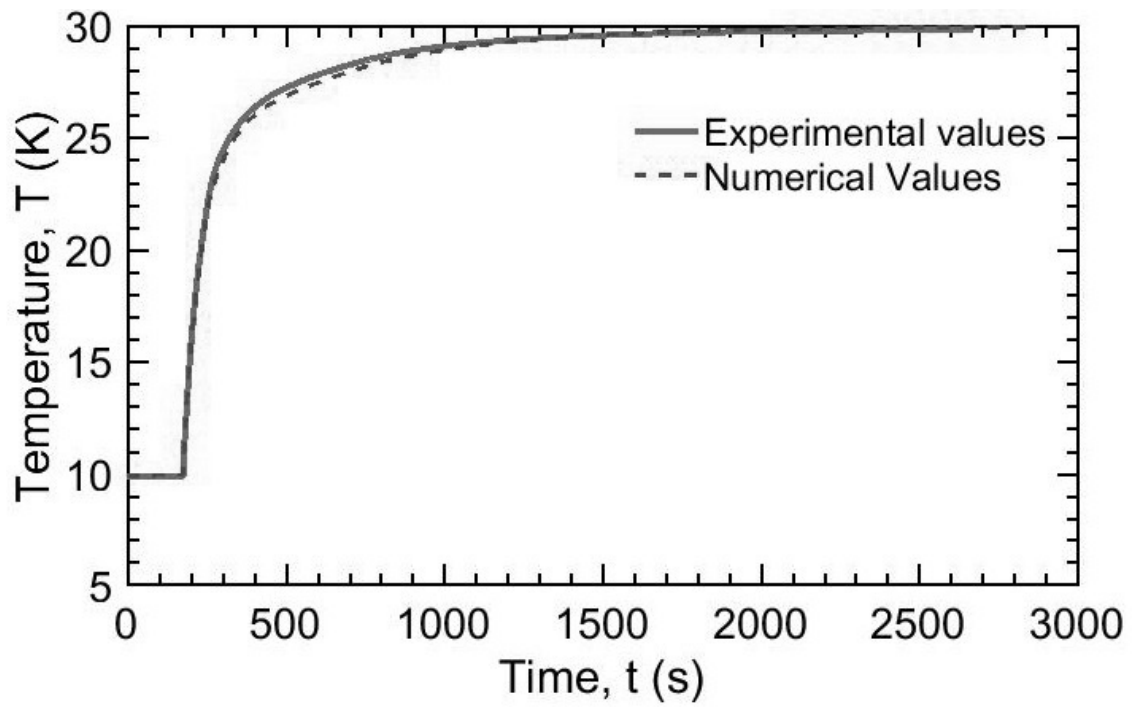

Figure 2.4: Experimental and numerical time response for the dry cell experiment with the $10 \mathrm{~mm}$ diameter 316L SS test cell.

balance between accuracy and computational speed.

\subsection{Liquid-Vapor Interface Shape Determination}

Preliminary testing indicated the possibility of the existence of a contact angle of approximately 10 degrees between liquid hydrogen and aluminum. The conical transition in the first test cell was designed to qualitatively probe the possible existence of a contact angle between hydrogen and aluminum. Figure 2.3(a) is a neutron radiograph of liquid hydrogen in the conical section of this test cell. The notable feature is the flatness of the liquid-vapor interface despite the Bond number being approximately 9. At this Bond number, the interface will have significant curvature in a 
circular cylinder as seen in figure 2.5. Figure 2.3(c) is the log transform of the normalized image. These images suggest the presence of a finite contact angle between liquid hydrogen and aluminum.

While the general consensus may be that cryogens are perfectly wetting, there are many studies that indicate wetting transitions and finite contact angles. Cheng et al. [38] investigated the wetting transition of liquid hydrogen on an alkali metal surface using a quartz microbalance. They confirmed the existence of a wetting transition temperature between $17.8 \mathrm{~K}$ and $18.0 \mathrm{~K}$ for a relative pressure of 1.0 . As the relative pressure decreased (less than standard pressure), the wetting transition temperature increased. The behavior of hydrogen and helium wetting transition was used by Herminghaus et al. 63] as a model system for investigation of wetting phenomena. The wetting transition was optically investigated using a Surface Plasmon Resonance (SPR) imaging system. Droplets of liquid hydrogen were observed to form on a homogeneous film absorbed to a gold film (used to generate the plasmons), which indicates a finite contact angle. Similarly, Ross et al. [112] observed the formation of isolated droplets of liquid hydrogen on cesium below the wetting transition temperature. They optically measured the contact angle of liquid hydrogen on an alkali metal surface below the wetting transition temperature, reported as $20.6 \mathrm{~K}$, and concluded that the liquid-solid interfacial energy is "not negligibly weak as has been assumed." There is also a strong posssibility that viscous stresses in the contact line region due to phase change may alter the apparent contact angle of a meniscus. Krahl et al. [79], 
using drop tower experiments and independent computational studies, developed a dimensionless correlation of the apparent contact angle shift with a temperature difference between the substrate and the liquid phase. The correlation is based on an apparent contact angle measured at a location on the meniscus where the liquid film thickness is $140 \mu \mathrm{m}$. Additional experimental support for the experimentally observed and computationally predicted shift in contact angle is reported in Kulev and Dreyer [82] and Fuhrmann and Dreyer [54]. Using the correlation of Krahl et al. [79], a 4 degree apparent contact angle for an evaporating hydrogen meniscus requires a $0.18 \mathrm{~K}$ temperature difference between the liquid and the test cell wall. This temperature difference is less than the uncertainty of the experimental measurements, but within the uncertainty of the computational predictions of the thermal model.

In order to more accurately determine if a finite contact angle exists, the meniscus profiles from the cylindrical sections of the four test cells are matched to the theoretical Laplace curves. Laplace curves are determined by numerically integrating the Laplace-Young equation (equation 2.2) for a axisymmetric cylinder in cylindrical coordinates [40].

$$
\frac{d f(\psi)}{d \psi}=\frac{\sin \psi}{\operatorname{Bof}-(\sin \psi) / r+\lambda}, \quad \frac{d r(\psi)}{d \psi}=\frac{\cos \psi}{\operatorname{Bof}-(\sin \psi) / r+\lambda}, \quad\left(0<\psi<\frac{\pi}{2}-\theta\right)
$$

$f(\psi)=r(\psi)=0$ at $\psi=0$ and $r(\psi)=1$ at $\psi=\pi / 2-\theta$. The origin of the coordinate system lies at the center of the meniscus. $f(r)$ is the dimensionless height of the 
surface at a dimensionless distance $r$ from the center, $\lambda$ is twice the curvature of the meniscus at the origin and Bo is the Bond number. $\theta$ is the contact angle between the meniscus surface and the cylinder wall and $\psi=\tan ^{-1}(d f / d r)$ is the angle between the meniscus and a horizontal plane. The equation is solved numerically in MATLAB using ode113 which is a variable order Adams-Bashforth-Moulton predictor-corrector solver. Once the appropriate Laplace curve is found for the hydrogen meniscus, the contact angle is the value of the first derivative of $f$ at $r=1$.

The noise in the images posed difficulties in accurately determining the meniscus shape, especially near the test cell wall. Different types of noise are present in the images, namely salt noise (bright pixels in different locations for different images) and Poisson noise (variance in pixel intensities about a mean). Salt noise is characterized by pixels with very high intensity values, with values ranging from few times $(2 \times$ to $3 \times$ ) to more than $40 \times$ higher than the adjacent pixels. These are removed by replacing the hot pixels with the median intensity of the surrounding pixels intensities. Poisson noise is difficult to attenuate. At the center of the test cell the meniscus location is more accurately identified due to larger gradients in the pixel intensities at the liquid-vapor interface. Moving radially from the center, the thickness of the liquid cross-section decreases with an accompanying decrease in signal-to-noise. The total exposure time for each image is 10 seconds.

Poisson noise is reduced by stacking multiple images and averaging the stacked image. 


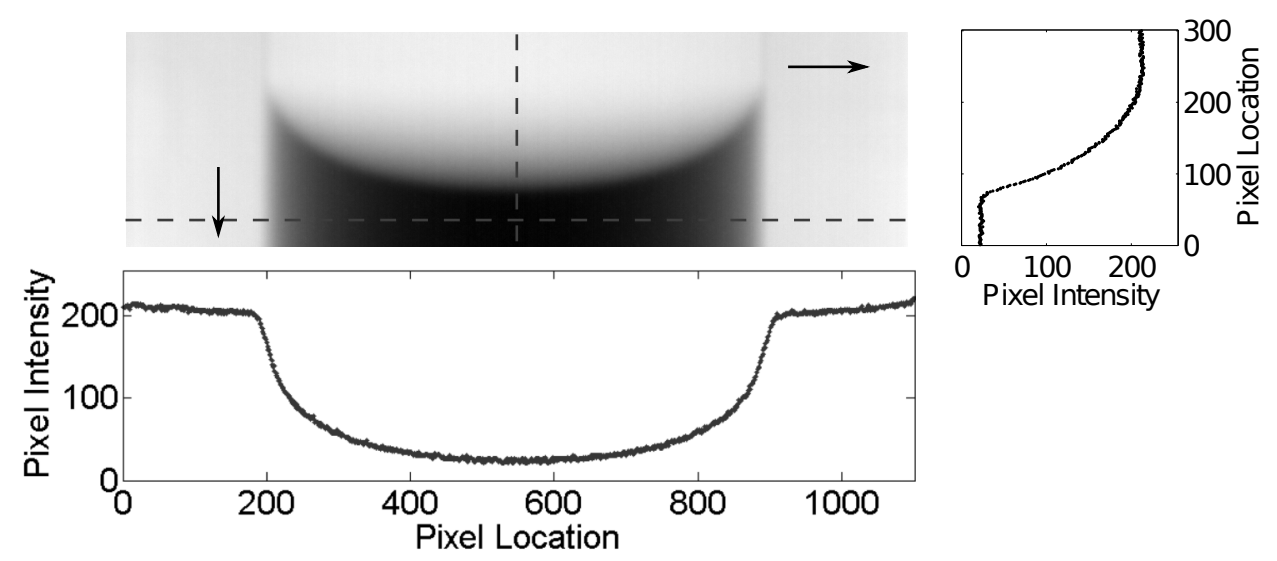

Figure 2.5: Stacked and averaged images with variation in pixel intensities along the highlighted row and column.

During evaporation or condensation, the meniscus is moving. Therefore, the image is re-centered for stacking. A sequence of stacked and averaged images is shown figure 2.5. The images have been cropped to $1101 \times 301$ pixels with an interior diameter of $10 \mathrm{~mm}$.

The meniscus shape is determined by examining the pixel intensity variation from the liquid to the vapor along vertical columns of pixels. In the liquid the pixel intensity remains relatively constant as shown in Figure 2.5. The pixel location at which the intensity drops below the average liquid intensity indicates the presence of the meniscus. The threshold for the limiting intensity is calculated by averaging the intensity of all the pixels in the vertical column constrained between the bottom of the test cell to the row corresponding to the meniscus apex. The average pixel intensity for each column of pixels varies from a minimum at the center of the test cell to a maximum at the test cell wall. Thus, the value of the limiting threshold increases 


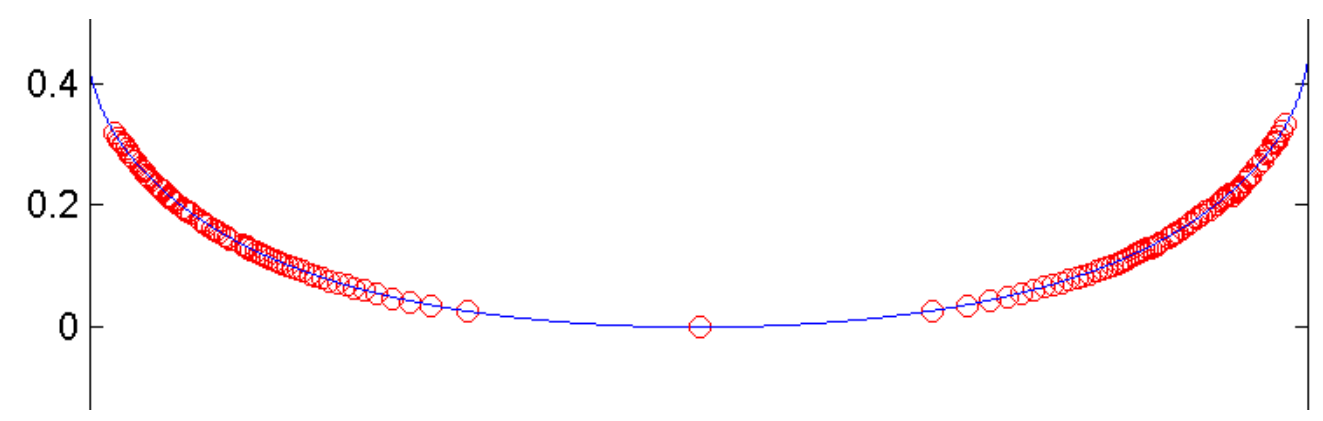

Figure 2.6: Laplacian curve fit to the detected meniscus edge on stacked image of liquid hydrogen in Aluminum test cell. Symbols are the meniscus edge and the line is the Laplace curve representing the best fit to the edge data.

automatically resulting in an adaptive threshold. The use of a fixed threshold is avoided as it alters the location of the meniscus based upon the value selected for threshold.

Once the meniscus edge is determined, the data is scaled, non-dimensionalized and fit to a Laplace curve. The error is calculated as the sum of the normal distance between the points on the meniscus and the Laplace curve [67]. The Laplace curve that results in the minimum error is the curve that represents meniscus. The resulting Laplace curve suggest that a contact angle of $4^{\circ} \pm 4^{\circ}$ for liquid hydrogen in the 10 mm diameter aluminum test cell. The relatively large uncertainty is the result of the image noise and the meniscus motion during imaging. The shift in meniscus position during a single 10 second exposure varied between 6 and 16 pixels depending upon the rate of evaporation or condensation. In addition, for very small contact angles the calculated contact angle is highly sensitive to the errors in the meniscus edge detection and the accuracy of meniscus edge detection to limited to \pm 3 pixels. 


\subsection{Summary}

A new method for imaging liquid hydrogen menisci undergoing phase change has been developed using the NIST Neutron Imaging Facility. The eventual objective is to couple the macroscale imaging to a computational thermal model and then to a microscale model of the contact line region in order to extract evaporation and condensation coefficients for liquid hydrogen. The methodology for conducting the neutron imaging experiments are discussed in detail and stable control of evaporation and condensation of hydrogen in aluminum and stainless steel cylindrical test cell was achieved. Neutron imaging experiments with liquid methane are being conducted at the time of writing this paper. The computational thermal model, necessary to predict the inner wall temperature distribution for the microscale model, accurately tracks the thermal transients of the experiment despite significant uncertainty in the mass of helium gas serving to convect heat to and from the test cell in the cryostat. Finally, initial observations suggest the existence of a non-perfectly wetting condition with hydrogen. 



\section{Chapter 3}

\section{Visualization of the Evaporation}

\section{and Condensation Phenomena in}

\section{Cryogenic Propellants}

\subsection{Introduction}

One of the key challenges in long duration space missions is the ability to store and predict evaporation and condensation of cryogenic propellants. The most commonly

Reprinted from Journal of Flow Visualization and Image Processing, Vol 23 by Bellur, Konduru, Medici, Hussey, Jacobson, LaManna, Allen \& Choi K, "Visualization of the Evaporation and Condensation Phenomena in Cryogenic Propellants", 137-156, 2016 with permission from Begell House, Inc. See Appendix A for documentation of permission to republish this material. 
used propellants are liquid hydrogen and methane that must be stored at temperatures as low as $20 \mathrm{~K}$ and $110 \mathrm{~K}$ respectively, under atmospheric pressure, to prevent boil off. The propellants are extremely sensitive to temperature/pressure variations and undergo phase change, even in space, resulting in self pressurization of tanks. Long term storage and transfer of propellants are mission critical technologies at NASA [41]. Computational fluid dynamics (CFD) models have been used to predict the pressure and behavior of the cryogens inside large tanks and it was found that a thin $(\approx 1 \mathrm{~mm})$ liquid film exists between the vapor and the solid phase [13, 99-102]. Several bubble point pressure and vapor breakthrough experiments conducted at NASA Glenn suggest that the understanding of local thermodynamics is critical and much of the uncertainty was attributed to phase change [60 62]. The type of phase change encountered here is different from pool boiling and depends on several other factors in addition to temperature and equilibrium vapor pressure. The curvature of the liquid meniscus near the contact line results in an anisotropy in the liquid stresses including a pressure jump at the interface due to the surface tension. The intermolecular forces between the solid phase and the liquid become important in this thin film and give rise to a net pressure drop (disjoining pressure effect). As a result of these factors, the evaporation is non-uniform and most of the evaporation occurs in the thin liquid film called the contact line region. For wetting fluids, it has been shown that $60-90 \%$ of the evaporation occurs in the contact line region [53, 98, 106, 127]. Hence, the experimental effort to measure evaporation must focus on visualization and accurate 
determination of the liquid-vapor interface, especially in the contact line region.

Curvature and disjoining pressure effects have been incorporated in some phase change models $[1,106,110,124]$, but the results involve fitting the model's results to experimental data. Accurate modeling is still a challenge due to the lack of reliable experimental data on controlled phase change of cryogenic propellants [3, 4, 99]. Controlled experimental evaporation/condensation tests of cryogenic propellants are difficult to perform due to the fact that conventional visualization techniques cannot be used to image liquids inside metallic containers. Cryogenic propellants such as hydrogen and methane undergo phase change at $20 \mathrm{~K}$ and $110 \mathrm{~K}$ respectively at atmospheric pressures. During evaporation, the pressure builds up and a sophisticated manifold with a control system is necessary to control both pressure and temperature inside the metallic container. Further, flash evaporation of highly combustible propellants such as hydrogen invoke safety issues that require extreme caution during experiment design.

In the present study, neutron imaging is used as a visualization technique to detect the condensed propellant inside cylindrical Al 6061 test cells. Propellant vapor is introduced at a preset pressure into a cryostat cooled by liquid helium and liquid nitrogen. Condensation is achieved in the test cell by dropping the cryostat temperature lower than the saturation temperature and subsequent evaporation is achieved by raising the cryostat temperature above the saturation temperature. These tests were 
conducted at the BT-2 neutron imaging facility at the National Institute of Standards and Technology (NIST) at Gaithersburg, MD. This article details the visualization technique employed to capture the liquid-vapor interface and image analysis to obtain the phase change rates.

\subsubsection{Neutron Imaging}

Using neutrons for imaging can be dated back to the 1940's [59] but it was during the advances in digital photography and image processing that it became popular as a reliable tool for non-destructive testing [78]. Although the resolution obtained from neutron imaging is currently limited to about $10 \mu \mathrm{m}$, the capability to easily record and manipulate image data for 3D tomography studies spurred the development of digital neutron imaging. Brenizer [26] provides an extensive review of neutron imaging from its conception to the present day. Neutrons have no charge and interact with matter through the strong nuclear force, and as a result have good penetrating power through most metals. Neutrons do not deposit appreciable energy into a sample(if the entire beam was absorbed, the energy is of order $\left.\mu \mathrm{W} / \mathrm{cm}^{2}\right)$. Neutron imaging is a radiographic technique similar to x-rays, which makes use of the differences in attenuation characteristics of different elements [58]. Neutron interaction with matter can be broadly classified in 3 ways: (1) scattering due to interaction with the sample atoms (2) absorption by the nucleus (3) transmission through the interstitial spaces 
between the atoms. Neutron scattering and absorption are characterized by cross sections $(\sigma)$. The cross section is a probability expressed in units of area that denotes the likelihood of the neutron interaction with the medium of interest (figure 3.1). Crossections can be defined for both absorption $\left(\sigma_{a}\right)$ and scattering $\left(\sigma_{s}\right)$. The intensity of transmitted neutrons obeys the Beer-Lambert law of exponential attenuation (equation 3.1).

$$
I=I_{0} e^{-\mu d}
$$

In equation 3.1, $I_{0}$ is the intensity of the incident beam, $\mu$ is the macroscopic cross section (also known as attenuation coefficient) given by $\mu=n \sigma, \sigma=\sigma_{a}+\sigma_{s}, n$ is the number density given by $n=\frac{\rho A}{M}, \rho$ is the density of medium, $A$ is the Avogadro number, $M$ is the molar mass and $d$ is the thickness of the medium. Thermal neutrons are almost transparent to many metals but are strongly attenuated by light atoms such as hydrogen, lithium and boron. Table 3.1 compares the scattering lengths and attenuation coefficients of hydrogen, aluminum, iron and carbon. All hydrogenated compounds such as methane also exhibit strong neutron attenuation. It is this difference in attenuation coefficients that allows for the visualization of the liquid propellant meniscus through an aluminum cylinder. Neutron imaging allows not only for qualitative measurements but also quantitative measurements of the meniscus shape. Neutron imaging has successfully been used in a variety of liquid detection 
applications such fluid transport in porous media [48, 72, 104], fuel cells [17, 80, 113] and heat pipes [39, 74, 128]. To the authors' best knowledge, these are the first known neutron images of cryogenic propellant phase change.

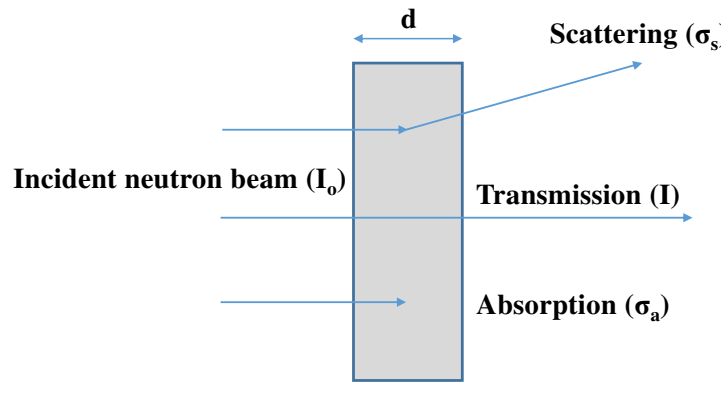

(a) Neutron interaction with matter

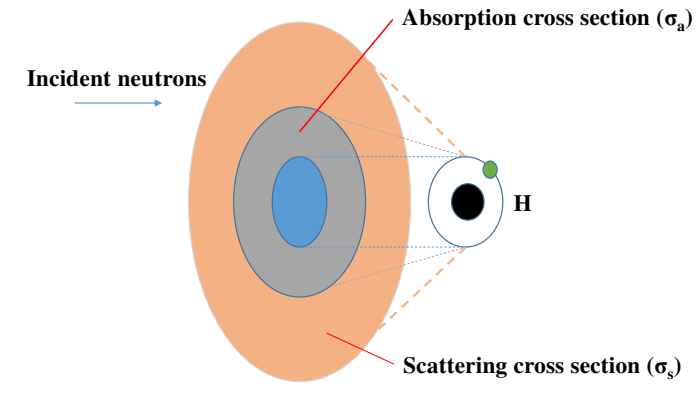

(b) Absorption and scattering cross sections

Figure 3.1: Neutron interaction with matter and cross sections

Table 3.1

Neutron cross sections and attenuation coefficients for $20 \mathrm{meV}$ neutrons [36]

\begin{tabular}{|c|c|c|c|}
\hline Species & $\sigma(\mathbf{b})$ & Density $\left(\mathrm{g} / \mathbf{c m}^{3}\right)$ & $\mu\left(\mathbf{c m}^{-1}\right)$ \\
\hline Hydrogen(liquid) & 33.75 & 0.0707 & 1.437 \\
\hline Hydrogen(vapor) & 33.75 & 0.0013 & 0.026 \\
\hline Aluminum & 1.34 & 2.7 & 0.083 \\
\hline Carbon & 5.02 & 2.25 & 0.566 \\
\hline
\end{tabular}

The objective of the current study is to establish a method to accurately determine the phase change (evaporation/condensation) rates by measuring the liquid propellant volume in every image. Two different methods are employed to measure volume: (1) Interface tracking method and (2) Optical density method. The interface tracking method involves using traditional image processing concepts such as spatial filtering and edge detection. The shape of the liquid vapor interface is determined and the interface is tracked as a function of time to calculate the liquid volume. The optical density method uses the beam hardness corrected Beer-Lambert law to transform 
every pixel's gray value into a liquid thickness from which the volume is computed.

\section{$3.2 \quad$ Experiment}

\subsubsection{Test setup}

A $70 \mathrm{~mm}$ bore cryostat was used to cool and eventually condense the propellant vapor introduced into the test cell. The cryostat consists of annular rings filled with cryogens with the test cell at the center in a space called the "sample well". The cryostat is cooled by an outer jacket of liquid nitrogen that is constantly boiling at atmospheric pressure maintaining a constant temperature of $77 \mathrm{~K}$. An inner jacket is filled with liquid helium that also undergoes phase change by throttling. The vaporization of liquid helium cools a copper heater block that in turn cools the cryostat's sample well. The vaporized helium then rises up the innermost annulus cooling the entire inner wall of the cryostat. The helium vapor is then either removed by a vacuum pump or vented to the ambient atmosphere depending on the cooling rate needed. The throttle valve and a helium exhaust valve serve as a means of controlling the vapor pressure and hence the cooling power of the cryostat. The cryostat is being cooled constantly as long as the cryogens are replenished. The copper heat exchanger has a temperature sensor and an electric heater embedded in it. Steady state operation is obtained by 
matching the electric heater power to the cooling power from the cryogens.

The test cell is mounted to a long hollow stainless steel sample stick by use of a custom fabricated SS 316 lid and inserted into the cryostat's sample well(figure 3.2). The lid has a 1/8" male VCR fitting brazed to its side to allow for connection to a vapor feed line. An internal groove then transfers the vapor from the feed line to through the lid and into the test cell. The lid is connected to the test cell by use of $6 \mathrm{Al} 4-40$ screws and an Indium O-ring. The entire setup and all fittings were helium leak checked prior to testing. The instrumented sample stick is inserted into the cryostat while the sample well is being flooded with helium vapor about $135 \mathrm{kPa}$. Helium flooding of the sample well is essential to prevent the introduction of ambient air along with the sample stick. Once the stick is inserted, it is secured with an O-ring seal and the sample well is pumped down to a pressure of $10^{-3} \mathrm{~Pa}$ to remove any traces of of other gases that might be present. Helium vapor is reintroduced into the sample at $135 \mathrm{kPa}$ and the process is repeated at-least 3 times. After the final evacuation, a small amount of helium is added to act as a heat exchange gas between the sample well and the copper heat exchanger/cryostat inner wall. The amount of helium added determines the transient thermal response between the heat exchanger and the test cell. During the time of the experiments, the final helium pressure in the sample well could not be measured. Numerical simulations are being conducted by the authors to characterize the thermal response and will be published in a future article. The stick contains several radiation baffles to prevent heat leaks by radiation from the top 
flange.

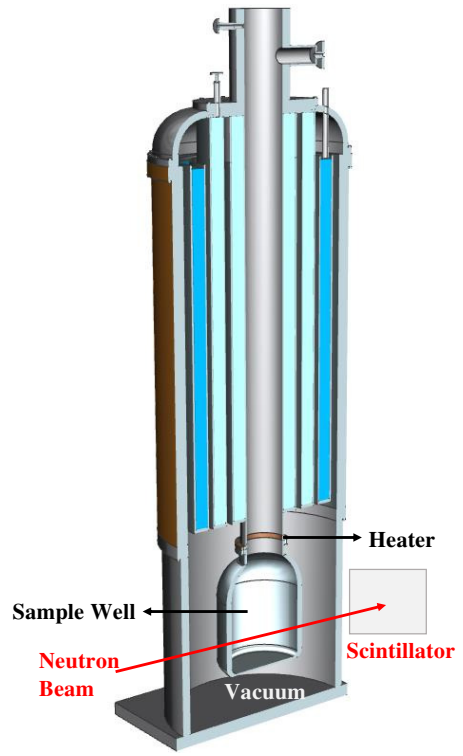

(a) $70 \mathrm{~mm}$ Cryostat

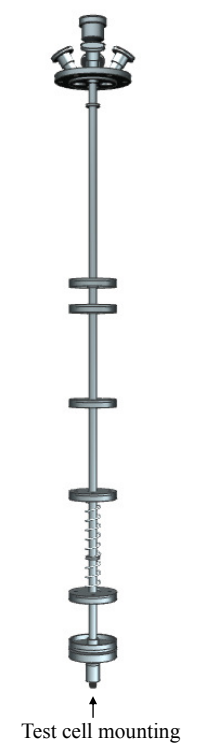

(b) stick

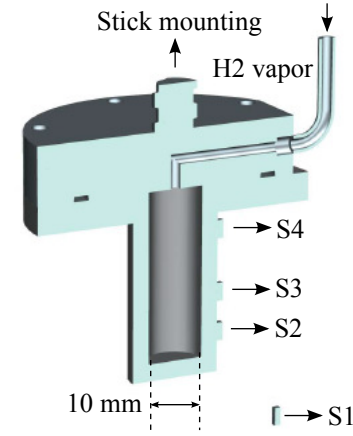

(c) $10 \mathrm{~mm} \mathrm{Al} 6061$ test cell

Figure 3.2: Test setup showing the instrumented test cell attached to the end of a long SS sample stick which inserted into the sample well of the 70 mm "orange cryostat".

Three Lakeshore* silicon diode DT-670 (S2-S4) sensors are mounted on the outside of the test cell by use of Kapton tape and were secured in place by use of custom made stainless steel springs. A fourth sensor (S1) is suspended in the helium exchange gas close to the test cell (figure 3.2). The DT-670 sensors were connected to a Lakeshore model 340 temperature controller for datalogging. The corresponding calibration curves for DT-670 sensors are built into the temperature controller. The temperature of the copper heat exchanger (NTC RTD X45720 sensor embedded in the heater housing) of the cryostat and the stick temperature (Si410B sensor embedded into the bottom of the stick) is logged by a Lakeshore model 331. Auto PID closed 
loop control is set up for the cryostat's copper block temperature. All tests were conducted at a $5 \mathrm{~W}$ maximum setting. The uncertainty in the DT-670 temperature measurements is $\pm 0.25 \mathrm{~K}$. The uncertainty in the cryostat heat exchanger temperature is $\pm 0.1 \mathrm{~K}$. The pressures are logged by two independent pressure transducers. One transducer (Mensor* CPG 2500) was connected to the feed line and the other transducer (Mensor DPG 15000) was connected to the manifold. The uncertainty in the pressure measurements were $0.2 \%$ at $100 \mathrm{kPa}$, increasing to $1 \%$ at $25 \mathrm{kPa}$. The computer used for data logging is a Windows* XP SP3 machine running Intel* Xenon X5550@2.67 GHz, 4 GB RAM and 2TB hard drive. The Lakeshore controllers and pressure transducers are connected to the logging computer via serial ports and data is logged every second. Additional details on the experimental setup can be found at [19].

\subsubsection{Imaging setup}

The sample stick-test cell assembly after being inserted into the cryostat is placed in the collimated neutron beam such that the center of the beam passes through the center of the test cell. A scintillator (screen that captures neutrons and emits photons along with gamma radiation) is placed downstream to detect neutron transmission (figure 3.2). The scintillator used was a $7.6 \mathrm{mg} / \mathrm{cm}^{2}$ Gadoxysulfide screen that had a thickness of $20 \mu \mathrm{m}$. The visible (green) light emitted by the scintillator is captured 
by a Andor NEO sCMOS (scientific Complementary Metal Oxide Semiconductor) camera with a pixel pitch of $6.5 \mu \mathrm{m}$ with variable exposure time. A $85 \mathrm{~mm}$ Nikon lens with a PK 13 extension tube was used to focus the scintillator light onto the camera. The experiments were conducted at the Neutron Imaaging Facility (NIF) NIST Center for Neutron Research (NCNR) in the BT-2 thermal beam line. Details on the NIF facility and the beamline layout can be found at $[68,69]$.

The generation of the neutron beam is an inherently random process, so that the statistics of the image formed by the neutron beam are well described by a Poisson distribution. The standard deviation of this distribution reduces with exposure time at the expense of temporal resolution [80]. An exposure time of $10 \mathrm{~s}$ was found to be an appropriate compromise for the phase change rates being tested and the liquid interface moves by no more than 15 pixels in two consecutive images. The image formation process is well described by pinhole optics and geometric blur arises as a result. Further, the detector resolution can limit the spatial resolution. The image can be enhanced by deconvolution with an estimated point spread function (PSF) determined from a sharp edge on the image as described by [68]. The estimated PSF and the results of deconvolution is discussed in a later section.

During the experiment, images are captured while saturated vapor at a constant pressure is introduced into the test cell and the cryostat temperature is lowered to achieve condensation of the propellant. When sufficient condensation is obtained, 
the cryostat temperature is then increased above the saturation condition to achieve evaporation. The imaging is stopped when all the liquid has evaporated. During every test, images were captured with the neutron beam turned off, in order to characterize the background radiation from the reactor. The attenuation coefficient of hydrogen is almost 56 times greater than that of aluminum. Hence the neutrons easily pass through the aluminum container but are then blocked by the hydrogen. The high neutron cross-section of the liquid propellant in comparison to both the containment vessel material and propellant vapor allows for the visualization of the liquid.

\subsection{Image Preprocessing}

The images obtained from the camera are stored as single precision 32 bit FITS images with an imaging array size of $2160 \times 2560$ pixels. The pixel gray values represent neutron transmission intensities as observed from the scintillator light. Figure 3.3 shows a condensation/evaporation test from January 2015. The hydrogen vapor was set at a constant pressure of $120.6 \mathrm{kPa}$ corresponding to a saturation temperature of $21 \mathrm{~K}$. Temperature of the cryostat was lowered to $19 \mathrm{~K}$ for condensation and increased to $23 \mathrm{~K}$ for evaporation. The dark region of the captured images represents low neutron transmission (liquid propellant) while the bright regions represent high neutron transmission (metals and propellant vapor). 


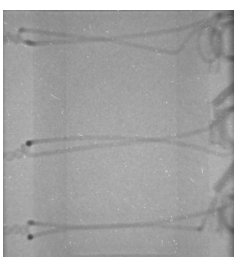

(a) $11 \mathrm{~s}$

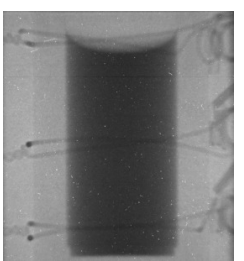

(e) $2654 \mathrm{~s}$

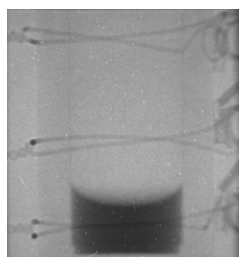

(b) $997 \mathrm{~s}$

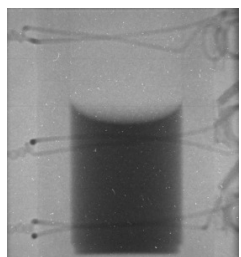

(f) $3316 \mathrm{~s}$

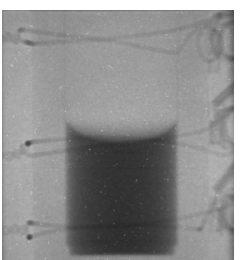

(c) $1218 \mathrm{~s}$

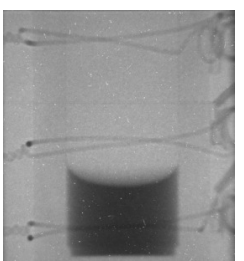

(g) $3867 \mathrm{~s}$

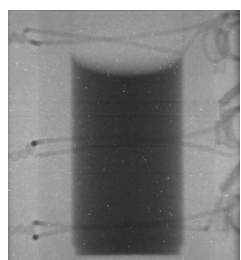

(d) $1438 \mathrm{~s}$

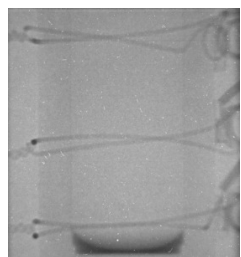

(h) $4475 \mathrm{~s}$

Figure 3.3: Time lapse images of liquid hydrogen phase change in a $10 \mathrm{~mm}$ Al 6061 test cell

Prior to using any method to determine the liquid volume, several parameters such as the location of the liquid-wall interface, pixel pitch and inherent rotation of the image must be determined and accounted in the forthcoming analysis. These preprocessing routines are common between both volume determination methods: interface tracking method and optical density method.

\subsubsection{Liquid-Wall interface}

Accurate determination of the liquid-wall interface location is vital for the curvature fitting with the interface tracking method and volume measurements with both methods. Assuming the outer wall of the cryostat and helium vapor does not significantly affect the neutron intensities, the attenuation due to thickness of liquid and the test 
cell wall can be modeled using the Beer Lambert's law (equation 3.11). The pixel intensities of the wall region are first fitted with an exponential model (equation 3.1). The results of this fit are then applied to the liquid region where the neutrons travel through both the test cell wall and the liquid. This is done using a two term exponential model where one term represents the test wall thickness and the other term represents the liquid thickness. The point of intersection of the liquid and the wall fits are evaluated as shown in figure $3.4(\mathrm{a})$. The plot represents exponential fits to the pixel intensities shown by the red line and arrow in figure $3.4(\mathrm{~b})$.

\subsubsection{Pixel pitch}

A correlation between the actual physical dimension and the pixel size is essential for any data analysis. Since the sample geometry was accurately machined and measured, it was used to calculate the effective pixel pitch. This is done by counting the number of pixels that make up the diameter of the test cell and scaling the obtained number by the measured diameter of the test cell. This was done for 10 images and at 5 different locations chosen at random on the test cell and then averaged. The effective pixel pitch was found to be $14.4 \mu \mathrm{m}$ per pixel for the January 2015 tests and $16.5 \mu \mathrm{m}$ per pixel for the September 2016 tests. Figure 3.4(b) shows the pixel pitch for the January 2015 setup. 


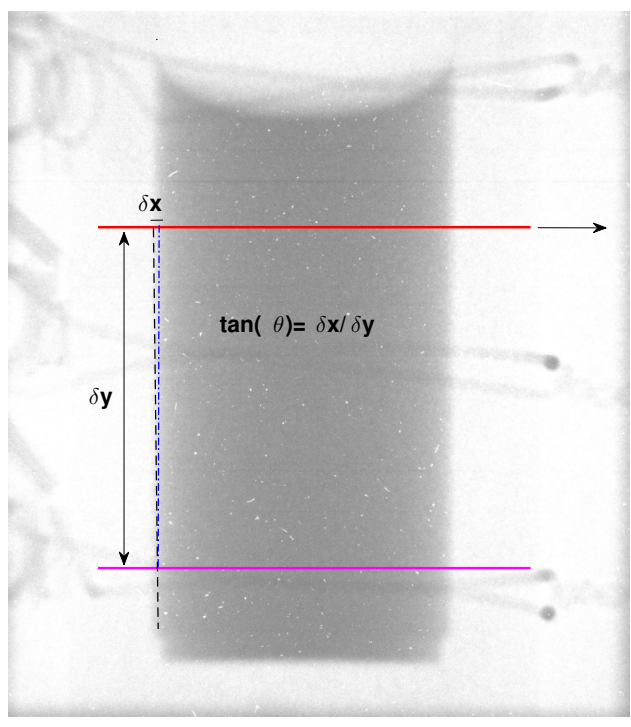

(a) Rotation determination

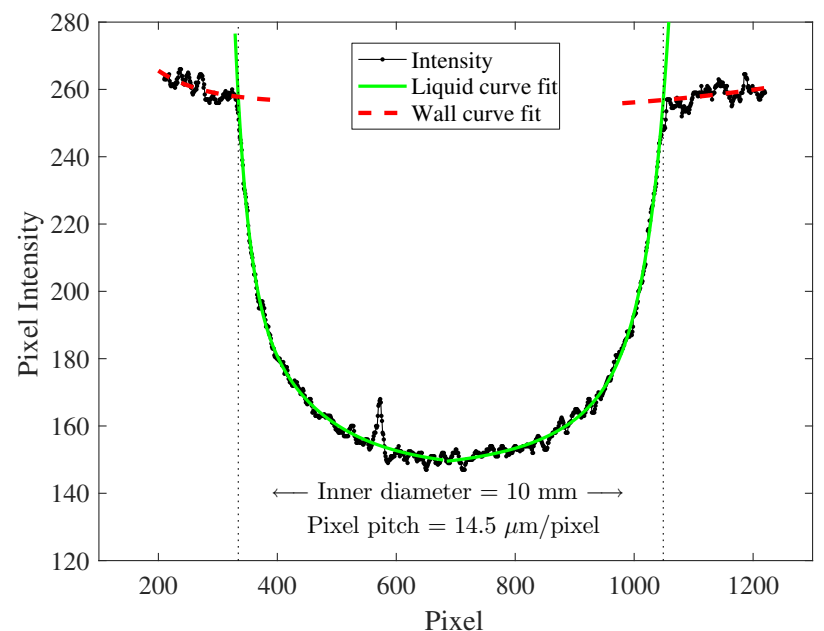

(b) Liquid-wall interface and pixel pitch determination

Figure 3.4: Determination of (a) rotation \& (b) liquid-wall interface and pixel pitch using pixel intensities shown by the red line and arrow. The image represents liquid hydrogen at $21 \mathrm{~K}$ in a $10 \mathrm{~mm}$ Al6061 test cell.

\subsubsection{Image Rotation}

In order for the interface tracking and the curvature determination to be accurate, the images containing the test cell and liquid meniscus must be as vertical as possible. An error of $0.5^{\circ}$ in rotation results in an increase of the corresponding volume measurement by up to $2 \%$ and the measured contact angle by up to $5^{\circ}$. The rotation was determined by analyzing images that have cryogens filled almost to the top of the vessel. The relative distance between the wall coordinates determined by analyzing points of the highest gradient was logged and measured for a sample of images. A correlation between the relative drift in the $\mathrm{x}$-coordinate $(\delta \mathrm{x})$, at various $\delta \mathrm{y}$ distances 
was obtained. The slope of the linear fit of $\delta \mathrm{x}$ vs $\delta \mathrm{y}$ is the tangent of the rotation angle. The rotation angle determined from image preprocessing is about $0.12^{\circ}$ for the the January 2015 tests and $0.41^{\circ}$ for the September 2016 tests.

\subsubsection{Deconvolution}

Assuming the test cell is perfectly aligned in the beam, the inner bottom center edge of the test cell was analyzed to determine the imaged width of the assumed sharp edge. This spread of pixel intensities was fitted to a Gaussian distribution and it was found that the standard deviation was about 3.5 pixels. Hence a symmetric Gaussian PSF with a standard deviation of 3.5 was used to deconvolve the images with 3 iterations of the Richardson-Lucy algorithm in MATLAB.

\subsection{Image Analysis}

\subsubsection{Interface Tracking Method}

The interface tracking method is an extension of the curvature measurement technique that involves an adaptive threshold edge detection and is validated by a fit to the theoretical Young-Laplace curve. Determining the liquid-vapor interface shape and 
tracking the interface in every image involves considerable spatial filtering in addition to the deconvolution to account for inherent noise in the neutron images. Although the image is a 32 bit image, it was determined that the pixel gray levels of importance are between 100 and 340. The original neutron images, however, contain several spots of intensities (gray levels) well above 20000 due to intrinsic noise in the camera or deposition of energy into the sensor from gamma rays. These intensities are essentially background noise and can be removed by using a spatial median filter. The pixel intensity histogram (figure 3.5) shows 3 peaks at about 160, 240 and 260 corresponding to liquid hydrogen, aluminum wall and hydrogen vapor. In order to avoid unnecessary blurring of the image instead of using a spatial median filter, intensities above 340 are set to 340 and all intensities were subtracted by 100 to remove the initial offset.

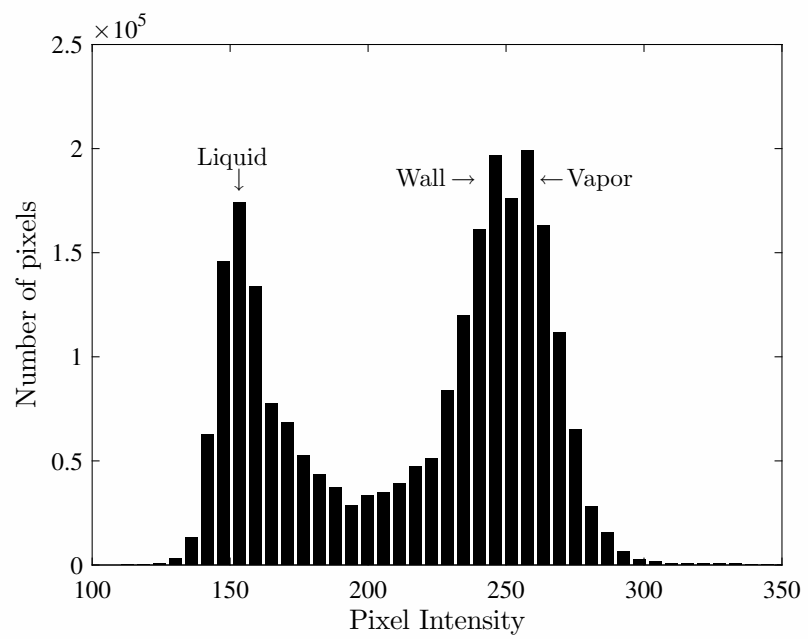

Figure 3.5: Histogram of pixel intensities for a typical liquid hydrogen in Al 6061 neutron image

Upon initial investigation it was observed that the curvature of the liquid-vapor interface does not change considerably with evaporation or condensation even though 
the contact line is moving. This is due to the slow phase change rates in the experiment in conjunction with the spatial resolution limits of the imaging setup. Any curvature change with contact line motion would require spatial resolution less than $5 \mu \mathrm{m}$. Hence, the signal to noise ratio in the captured images could be enhanced by image stacking. This is done by tracking the lower apex of the meniscus and cropping out the liquid-vapor interface in every image. The cropped images are then aligned, stacked and averaged to remove gamma noise. Salt noise was removed by using a $5 \times 5$ median filter prior to stacking. The final image obtained (figure 3.5) has high signal to noise ratio so that edge detection and a Laplace fit can be performed.

The liquid-vapor interface has a three dimensional curvature with contribution from both the in plane and the through plane components. Hence, a $1 \mathrm{D}$ edge detection does not suffice. The liquid-vapor interface region looks smeared because the image is essentially a shadow graph and the intensity reduces exponentially with film thickness and eventually becomes smaller than the resolution of the detector system. The pixel intensities change in both directions of the obtained 2D image. Variation in the vertical direction is due to reducing film thickness and intensity changes in the horizontal direction are due to the cylindrical geometry of the sample. In such a case, if a constant limiting intensity threshold is used, it would result in an erroneous meniscus shape. In order to overcome these challenges, a unique curvature determination is employed using an adaptive threshold. The preprocessed parameters along with the stacked images are used in a preliminary 1D vertical scan to obtain the first guess of 
the liquid-vapor interface. Based on the initial scan, the 2D meniscus is divided into 3 zones for processing: (1) a central zone where the curvature variation is small owing to a flat interface in the center, (2) an intermediate zone, where the $2 \mathrm{D}$ curvature changes significantly and (3) a corner zone where the curvature variation drops again and the film thickness is lower than $\approx 2 \mathrm{~mm}$. The image is sliced vertically and the three zones are analyzed separately (figure 3.6).

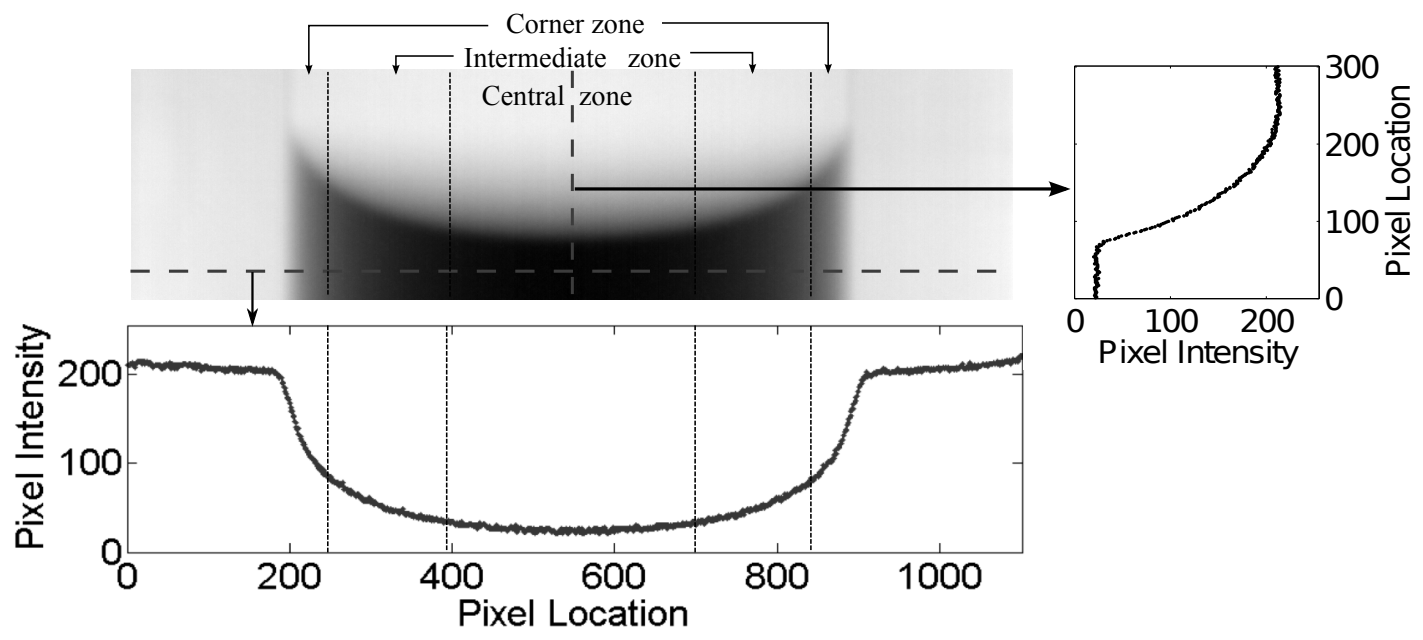

Figure 3.6: Stacked image showing the three zones of analysis, horizontal and vertical intensities along the centerline

All the pixels in the central zone are processed through a vertical 1D median filter of size 10 and a vertical 1D sgolay filter of size 10 in MATLAB and then averaged to remove any remaining neutron noise. A 1D vertical scan is made along every pixel column to find the first pixel location whose intensity is equal to the average intensity of the liquid region pixels in the same column. Hence, the limiting intensity constantly changes and results in an adaptive threshold. This adaptive threshold method accounts for the horizontal intensity smear as one gets closer to the wall. A quadratic 
curve fit to this edge provides a more accurate representation of the meniscus in the central zone. The ends of the quadratic fit serve as initial parameters for the intermediate zone left of the central zone. A diagonal edge detection is performed by scanning in both the $\mathrm{x}$ and $\mathrm{y}$ coordinates to find pixel positions that have intensities equal to the average intensity of all the liquid region pixels in the particular column directly underneath the current $\mathrm{x}$ range. Hence a modified adaptive threshold method is used in the intermediate zone to account for both the vertical and horizontal intensity distribution. The end point of the intermediate zone serves as an initial point for the left corner zone. The corner zone is analyzed using a 1D horizontal scan and the adaptive threshold method. The process is repeated for the right intermediate and the right corner zones. The resulting pixel location and intensities are extracted and a cubic spline fit is performed to ensure continuity between the zones. The final edge detected interface is shown in figure 3.7 .

The obtained edge detected interface is scaled and curve fitted to a theoretical YoungLaplace curve for validation. The Young-Laplace equation for an axisymmetric cylinder in cylindrical cordinates is given by equation 3.2 [40].

$$
\frac{d f(\psi)}{d \psi}=\frac{\sin \psi}{\operatorname{Bof}-(\sin \psi) / r+\lambda}, \quad \frac{d r(\psi)}{d \psi}=\frac{\cos \psi}{\operatorname{Bof}-(\sin \psi) / r+\lambda}, \quad\left(0<\psi<\frac{\pi}{2}-\theta\right)
$$


Additional conditions at the ends are given by $f(\psi)=r(\psi)=0$ at $\psi=0$ and $r(\psi)=1$ at $\psi=\pi / 2-\theta$. The apex is taken as the origin of the co-ordinate system. $f(r)$ is the dimensionless height of the interface at a dimensionless distance $r$ from the apex, $\lambda$ is twice the curvature of the interface at the origin, Bo is the Bond number, $\theta$ is the contact angle and $\psi=\tan ^{-1}(d f / d r)$. The equation is solved numerically using ode113 (a variable order Adams-Bashforth-Moulton predictor-corrector solver in MATLAB. The equation is evaluated for a variable curvature at the origin and a least squares fit is performed. Once the appropriate Laplace curve is found for the interface, the contact angle is the value of the first derivative of $f$ at $r=1$. Figure 3.7 shows the edge detected curve in comparison with the fitted Young-Laplace curve. The analysis presented here suggests a contact angle of $0-4^{\circ}$ for hydrogen in contact with aluminum oxide. A description of the possible existence of a contact angle and the errors involved with measurement is discussed in [19, 22, 77].

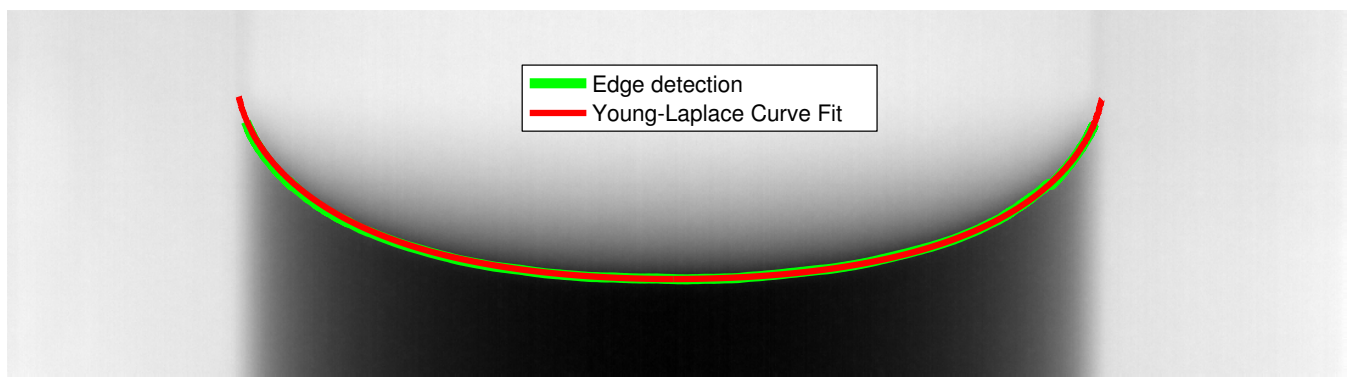

Figure 3.7: Edge detected and Young Laplace curves of liquid hydrogen in a $10 \mathrm{~mm} \mathrm{Al}$ cell at $19.9 \mathrm{~K}$

Within the limits of the imaging resolution, the curvature of the liquid vapor interface does not change during phase change. Hence the shape of the interface from the 
Laplace fit and the height of the meniscus apex from the inner bottom of the test

cell are the only two parameters needed for accurate volume determination. All neutron images from a test run are processed through a $[5 \times 5]$ median filter and a $[5 \times 5]$ sgolay filter in MATLAB to remove stray neutron noise. Once a full meniscus is formed after condensation, the central scan described above is repeated on the entire image. The minimum of the quadratic fit provides the apex location needed for the volume determination. An axisymmetric 2D shape is constructed with the Laplace fit displaced by the apex height obtained for each image. The area and centroid of this $2 \mathrm{D}$ shape is determined using the trapezoidal rule and the volume is determined using Pappu's second centroid theorem. The process is repeated for each image and the volume at every timestamp is logged.

\subsubsection{Optical density method}

The optical density method converts every pixel intensity (gray value) into a liquid thickness and thus relies on the intensity resolution. Every image with liquid is normalized with a dry image of the test cell (prior to condensation), the background image is removed and an attenuation coefficient for the liquid is measured. The coefficient is then used to transform the pixel intensities of the normalized image to a liquid transmission thickness using a modified Beer-Lambert equation. The liquid transmission thickness of the entire image is summed and multiplied with the pixel 
pitch to obtain the total volume.

In the images of the test cell just prior to condensation and just after evaporation, there is no visible liquid in the test cell but vapor at a set pressure is present. The attenuation from propellant vapor is not sufficient for visualization. These images are median combined to form a "reference" image so that the only difference between the reference image and the test image is presence of the liquid propellant. Images with the neutron beam turned off was used characterize the background radiation from the reactor. These images are median combined as well to form a "background" image.

As in the interface tracking method, further denoising routines are needed in addition to the deconvolution to remove the gamma noise. In this method, one must preserve most of the original pixel intensities and not intentionally blur the image with spatial filters to obtain a true volume from the original pixel intensities. Gamma noise or hot spots are randomly formed on every image and a temporal running median filter with a window of 3 images is used to remove them. Since the interface moves by less then 15 pixels in every test, a window of 3 images ensured that the temporal resolution is not severely affected. The filter is constructed such that the pixel intensities of a previous image, current image and next image is compared and a median value is chosen as the pixel intensity of the current image. This process is repeated for every pixel of every image of the test. The resulting images are mostly free of gamma noise and erratic jumps in intensity while preserving both spatial and temporal detail. 
The processed neutron images from the phase change test $\left(I_{T E S T}\right)$ are converted into an optical density image $\left(I_{O D}\right)$ by removing the background $\left(I_{B}\right)$, normalizing resulting image with the reference image $\left(I_{R E F}\right)$. A logarithmic transform of the resulting normalization is the optical density image (equation 3.3). A sample optical density image of condensing hydrogen $($ temperature $=19 \mathrm{~K}$, pressure $=120.6 \mathrm{kPa})$ in a $10 \mathrm{~mm} \mathrm{Al} 6061$ cell during the January 2015 tests is shown in figure 3.8 .

$$
I_{O D}=-\log \left(\frac{I_{T E S T}-I_{B}}{I_{R E F}-I_{B}}\right)
$$

In order to account for the polychromatic neutron beam at the NIST BT-2 facility, a beam hardening correction must be applied to the exponential attenuation law to convert the optical density image into a liquid transmission thickness image (equation 3.4).

$$
O D=\mu d+\beta d^{2}
$$

The coefficients to equation $[3.4, \mu$ and $\beta$ are calculated from the sample optical density image by considering the horizontal line profile of optical density values along the condensed liquid (solid green line in figure 3.9). Assuming the test cell to be a perfect cylinder, the geometric thickness of the liquid (neutron transmission distance) 
can be calculated. The measured optical density is then fitted to the liquid thickness using the beam hardening model (equation 3.4) to obtain the attenuation coefficient $\mu$ and beam hardening correction factor $\beta$ for the particular test run. Figure 3.9 shows a line profile of optical densities along with the the geometric liquid thickness and the results of a beam hardening fit. Figure 3.10 shows the almost linear variation of optical density with liquid thickness with a slight second order beam hardening correction. At low liquid thickness $(<0.2 \mathrm{~mm})$ the fit is bad due to the spatial resolution of neutron imaging combined with the quadratic nature of the film geometric film thickness. $\mu$ and $\beta$ are weak functions of density and since pressure changes by less than 5kpa during the course of an condensation/condensation test, the coefficients are assumed to remain constant and are only calculated once per test run. The attenuation coefficient calculated from optical density images for liquid hydrogen at 120 $\mathrm{kPa}$ is 1.431 which compares very well with the theoretically calculated coefficient of 1.437 (Table 3.1).

The calculated coefficients $(\mu$ and $\beta$ ) are then used to estimate a liquid transmission thickness value from every optical density pixel value using equation 3.4. The volume of liquid at each pixel is calculated based on each pixel's liquid transmission thickness and the pixel pitch. The volume of liquid in the entire image is summed based on the individual volumes represented by each pixel. Phase change rate can then be computed based on the liquid value from every image. 


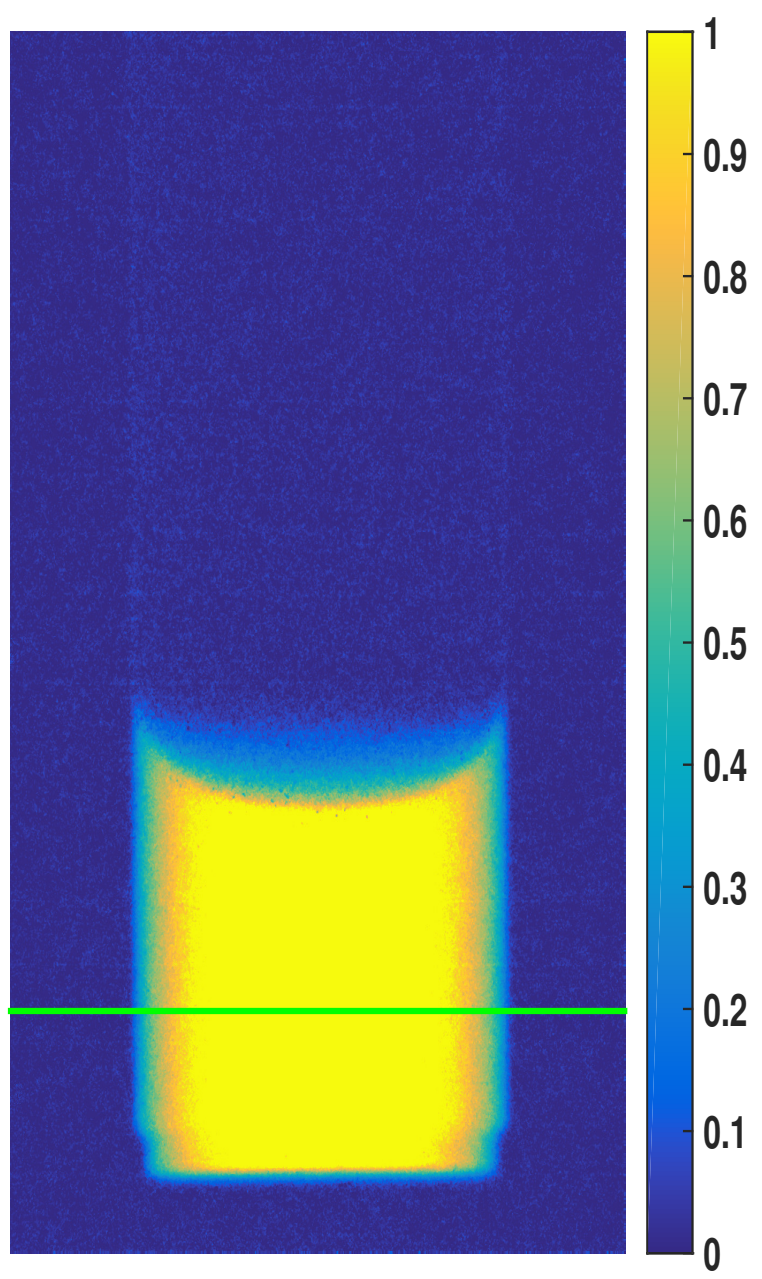

Figure 3.8: Sample optical density image of condensed liquid hydrogen in a $10 \mathrm{~mm} \mathrm{Al} 6061$ cell from the January 2015 test

\subsection{Results and Discussion}

Two different methods of measuring the volume of condensed liquid is discussed in the previous section. While the optical density method is robust to calculate volume from all images, the interface tracking method needs a fully formed meniscus for 


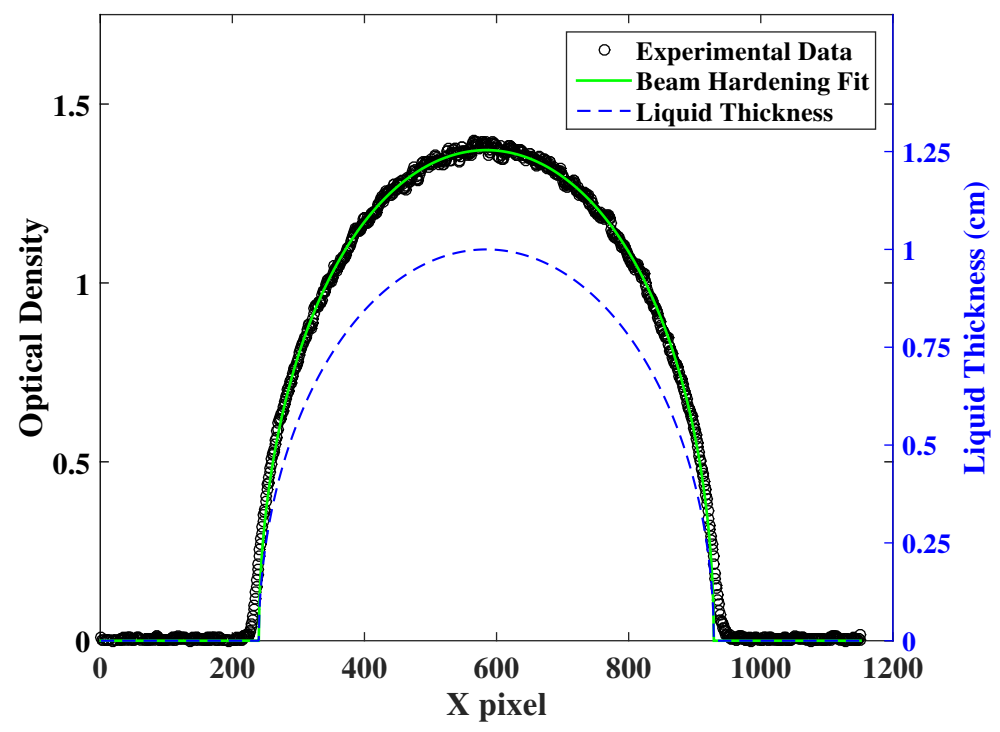

Figure 3.9: Line profile of optical density along the liquid along with geometric liquid thickness (solid green line in figure 3.8)

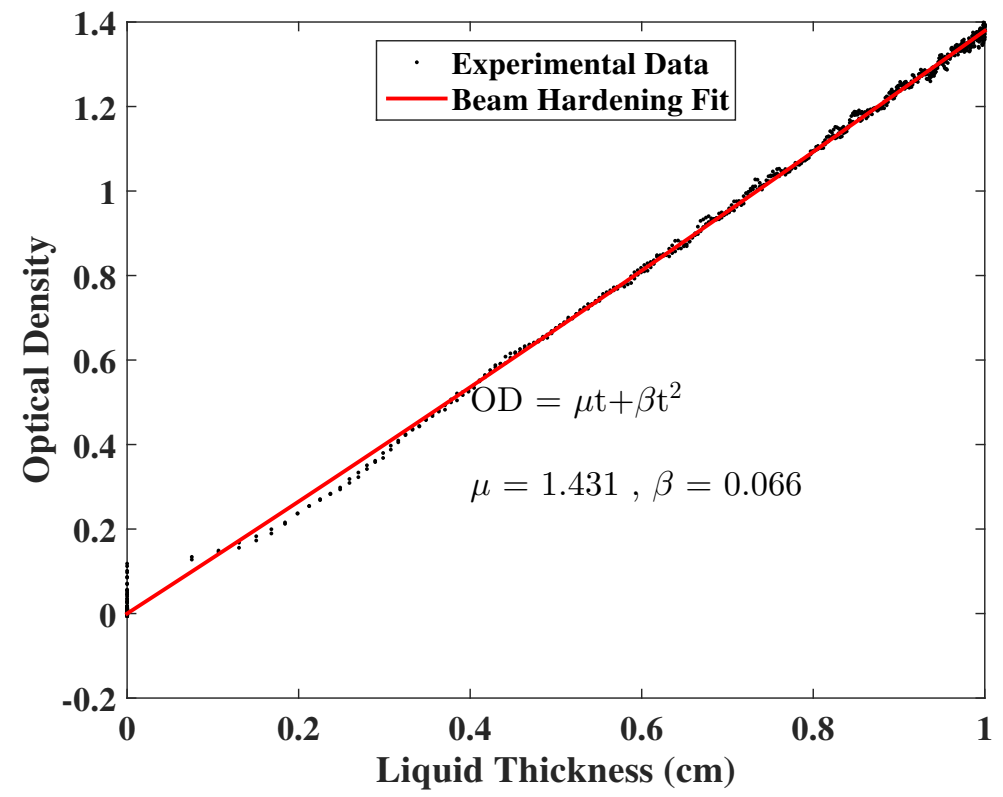

Figure 3.10: Beam hardening fit to calculate attenuation coefficient

its use. Further, it was noticed in the September 2015 images with methane that condensation is seen on the top corner of the lid. The interface tracking method 
cannot compute the volume of this corner section and thus is only applicable when a single fully formed meniscus is seen. Condensation on the top corner was not captured in the January experiments due to the field of view of the imaging setup. The YoungLaplace fits in the interface tracking method suggests the possible existence of a finite contact angle. However, the optical density method shows that there exists a thin film of propellant on the walls of the test cell at all times. Figure 3.11(a) shows a sample image from the September 2015 test with condensed methane at $117.2 \mathrm{kPa}$ and $110 \mathrm{~K}$ and figure $3.11(\mathrm{~b})$ shows the line profile corresponding to the dashed red line. Condensation on the top corner is clearly observed and the line profile shows two clear peaks near the wall and an offset in optical density at the center of the cell. Both these features indicate a thin liquid film on the wall. The average thickness of the film in figure 3.11 is estimated to be $15 \mu \mathrm{m}$. It is the authors' hypothesis that the film thickness is a function of the condensation/evaporation rate and this will be explored in a future publication.

The temporal resolution of the measured volume is estimated to be $\pm 15.1 \mathrm{~mm}^{3}$ due to the image exposure time of 10s. This estimate is based on the maximum movement of the liquid meniscus ( $\approx 15$ pixels) in consecutive images during the experiment. This temporal uncertainty applies to liquid volume determined by both methods described. Volume determination from the interface tracking method has additional uncertainty from edge detection, estimated to be a maximum of $\pm 5.7 \mathrm{~mm}^{3}$ due to a \pm 5 pixel error in interface detection. The errors add in quadrature and total uncertainty in 


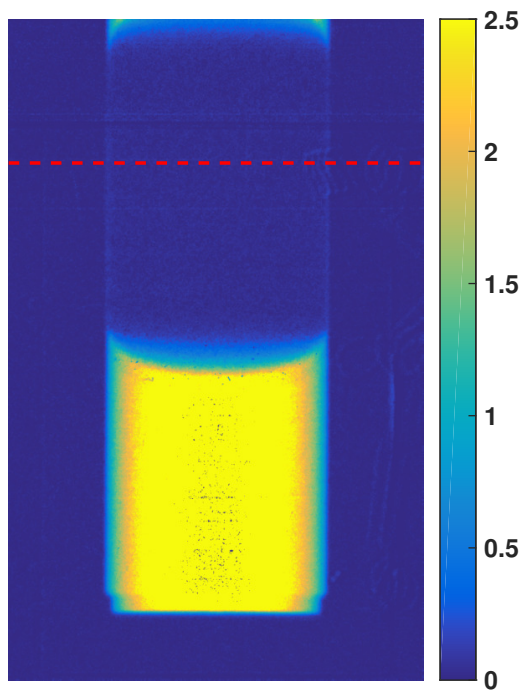

(a) Sample optical density image

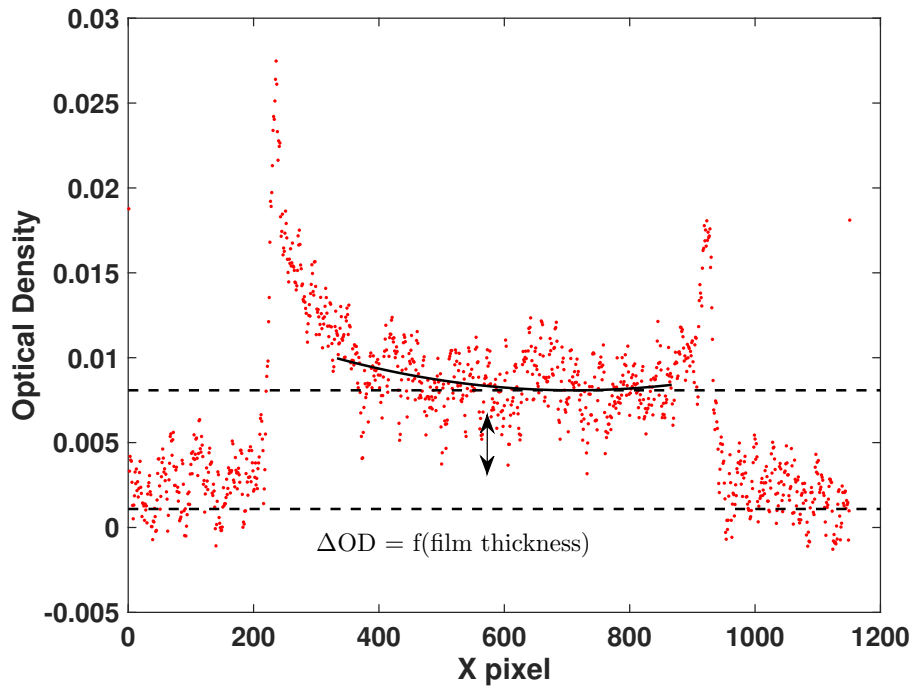

(b) Line profile scan

Figure 3.11: Sample optical density image of condensed methane at 110 $\mathrm{K}$ and $117.2 \mathrm{kPa}$, from the September 2015 test (a) and the corresponding line profile scan along the dashed red line

the interface tracking volume measurement is $\pm 16.14 \mathrm{~mm}^{3}$.

An additional uncertainty in the optical density method is based on neutron counting statistics in addition to the temporal uncertainty. Neutron transport to the imaging detector is a random process and the number of neutrons at a given time in a detector pixel is described by a Poisson distribution [68]. Ignoring the beam hardening correction and the uncertainty in the linear attenuation coefficient, the error in liquid volume from optical density can be estimated by equation 3.5 .

$$
\delta v=\frac{1}{\mu} \sqrt{\frac{2 N}{I_{o} A T \eta}}
$$


where, $N$ is the number of pixels used to calculate the volume, $I_{o}=5 \times 10^{6} \mathrm{~cm}^{-2} \mathrm{~s}^{-1}$, the incident fluence rate, $A$ is the area of each pixel, $T$ is the exposure time and $\eta$ is the detection efficiency ( 0.8 for the GadOX screen used). The random error in the volume measurement with optical density method calculated from equation 3.5 is $\pm 0.56 \mathrm{~mm}^{3}$ and the total uncertainty is $\pm 15.11 \mathrm{~mm}^{3}$. Uncertainty in the volume measured from the edge detection method is about $6 \%$ greater than the volume uncertainty in the optical density method.

Figure 3.12 shows the results from one such test with hydrogen at saturated at $21 \mathrm{~K}$ in a $10 \mathrm{~mm} \mathrm{Al} 6061$ cell. The result corresponds to time lapse images in figure 3.3 , Volume of liquid in the test cell based on neutron images is computed using both the interface tracking method and the optical density method and the agreement is very good.

\subsection{Summary}

A new experimental technique to observe the liquid-vapor interface during phase change of cryogenic propellants is presented. Using the $70 \mathrm{~mm}$ orange cryostat and the BT-2 Neutron imaging facility at NIST, Gaithersburg, controlled phase change tests of liquid propellants were conducted and images of the liquid inside the opaque metallic containers were obtained through neutron transmission imaging. A new technique 


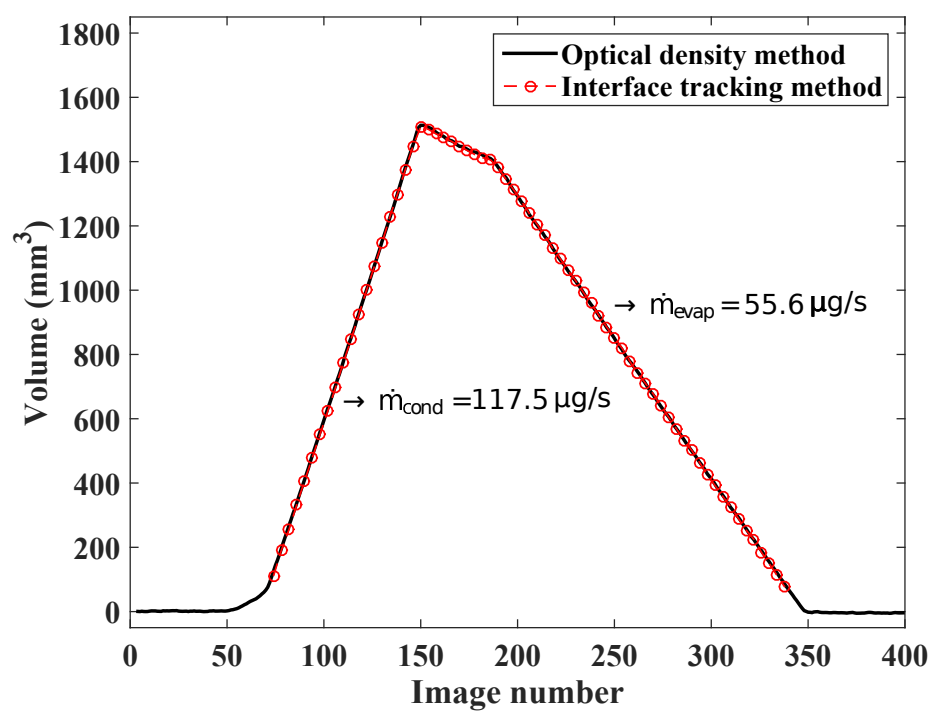

Figure 3.12: Phase change rates for hydrogen in the $10 \mathrm{~mm} \mathrm{Al}$ cell saturated at $21 \mathrm{~K}$. Condensation test is conducted at $19 \mathrm{~K}$ and evaporation at $23 \mathrm{~K}$

to accurately identify the liquid-wall interface has been determined using a simple exponential attenuation law. Two methods to determine condensed liquid volume are discussed and results are compared. The interface tracking method uses edge detection on a stacked image to compute the liquid vapor curvature and eventually measure the volume. The optical density method transforms every pixel gray value into liquid thickness and computes volume. Despite the fact that interface tracking method makes an assumption that the interface curvature is constant during a test, the measured volume agrees very well with the optical density measurement of volume. The volume measurement error with the interface tracking method is $\pm 16.14 \mathrm{~mm}^{3}$ while the volume measurement with the optical density method is $\pm 15.11 \mathrm{~mm}^{3}$, about $6 \%$ lower. The interface tracking method cannot be used unless a full meniscus is 
formed while the optical density method can work on any image. Further, the optical density technique can detect thin liquid films on the cell wall while the interface tracking method cannot. Hence, for an estimation of phase change rates from simple steady evaporation/condensation processes, an interface tracking method is shown to suffice but for high accuracy volume measurements and thin film analysis the optical density method should be preferred. 


\section{Chapter 4}

\section{Determining solid-fluid interface}

\section{temperature distribution during}

\section{phase change}

\subsection{Introduction}

One of the limiting factors in long duration space missions is the ability to maintain propellant storage depots. Computational Fluid Dynamics (CFD) along with a lumped parameter treatment of the vapor has been used to study pressurization in

The material contained in this chapter was previously published in Cryogenics by Bellur et al. [20]. See Appendix A for documentation of permission to republish. 
cryogen tanks and these have shown that a thin $(\approx 1 \mathrm{~mm})$ liquid layer separating the vapor phase from the wall is obtained [13, 100 102]. Propellants exist as liquid-vapor mixtures that constantly undergo phase change. Liquid-vapor phase change is a complex, multi-scale problem and kinetic theory has provided the framework for modeling evaporation/condensation for over a century. Classical kinetic theory is a statistical description of the behavior of gases based on velocities of the constituent molecules. Although kinetic models have shown to be very effective in capturing phase change, the use of the models is still limited due to the fact that kinetic theory only describes the maximum phase change flux possible for a given thermodynamic situation [64]. In reality, the phase change flux may be lower than the maximum value depending on the molecular species under consideration [116]. Evaporation and condensation coefficients were introduced by Knudsen [75] in order to account for the deviation from the maximum phase change rate. Evaporation and condensation coefficients are often set equal to each other and referred to as the accommodation coefficient. CFD modeling of propellant behavior utilizes the accommodation coefficient as an input to capture phase change [14, 15, 101]. This is particularly challenging due to the lack of available evaporation/condensation coefficients and the inability to sufficiently resolve local thermodynamics at the liquid-vapor interface [3, 4, 19, 99]. These coefficients must be determined experimentally [1].

At an evaporating or condensing meniscus, the normal stress in the bulk liquid is primarily influenced by interface curvature. Far from the meniscus, the adsorbed region 
consists of a nanoscale, non-evaporating layer of liquid molecules where intermolecular forces dominate. Between these two exists a transition film region in which the normal stress is affected by both intermolecular forces and interface curvature. For non-polar/wetting liquids, 60-90\% of the evaporation occurs in the thin film region close to the wall [43, 47, 53, 65, 106, 108, 127].

Most thin film evaporation models use a constant wall temperature condition in the transition film region [2, 25, 42, 43, 53, 56, 94, 108, 115, 127]. However, due to non-uniform evaporation there exists temperature gradients along the wall near the transition region as demonstrated by Stephan and Busse [120]. The non-uniform wall temperature can generate thermocapillary stresses at the interface, which in turn affects the local normal stress in the liquid and subsequently the evaporation rate. In order to accurately capture the thermophysics of evaporation at a contact line, thermal boundary conditions should be representative of local temperature distributions along the solid-liquid interface. Accounting for the non-uniform wall temperature is a key factor to accurately model phase change and ultimately calculate the evaporation and condensation coefficients. Details of calculation of evaporation and condensation coefficients using kinetic theory are described elsewhere [19].

This paper represents one piece of the overall methodology; a thermal model that serves to bridge the macroscale experiment observations with the micro-scale phase change modeling. The goal of this thermal model is (1) determine the rate and mode 
of heat transfer to the cryogenic liquid in the test cell and (2) translate discrete exterior surface temperature measurements to an interior wall temperature distribution suitable for use in the microscale transport model.

\subsection{Cryogenic Phase-Change Experiments}

Cryogenic phase change experiments with hydrogen and methane were conducted, using a 70-mm-cryostat at the Neutron Imaging Facility at the National Institute of Standards and Technology (NIST). The experiments were conducted at absolute pressures between $100-210 \mathrm{kPa}$, corresponding to saturation temperatures between $15 \mathrm{~K}-30 \mathrm{~K}$ for hydrogen and $100 \mathrm{~K}-120 \mathrm{~K}$ for methane.

Figure 4.1(a) illustrates the components of the cryostat. Test cells are suspended in a sample well, below the cryostat using a 720-mm-long sample holder. The test

cell is mounted to the bottom of the sample holder via a flange that includes a gas exchange port to allow hydrogen or methane vapor to be introduced into the test cell. The flange is attached to the test cell using an indium seal and secured in place with six screws. The sample holder is sealed at the top of the cryostat with ports for sensor leads and a cryogen vapor feed line. Radiation baffles on the sample holder minimize heat transfer from the top of the cryostat. The temperature in the sample well is controlled using a combination of an electric heater and liquid helium phase 


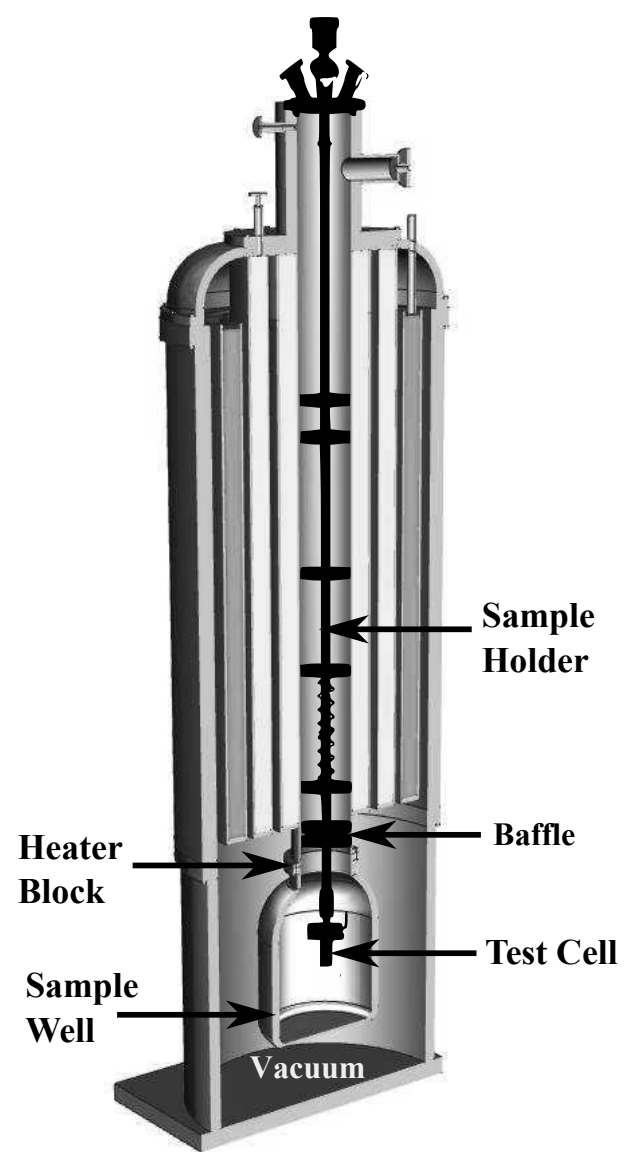

(a) Cryostat configuration

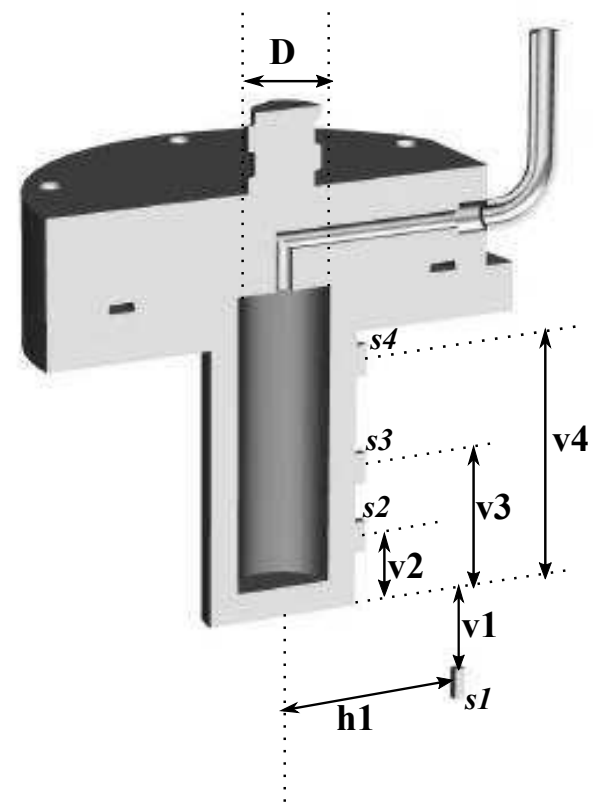

(b) 10-mm diameter test cell with sensor location

Figure 4.1: Hardware configuration for cryogenic phase change experiments. (a) Cryostat with test cell suspended in sample well. (b) Illustration of the 10-mm diameter test cell. s1, s2, s3, and s4 are the temperature sensors. The location of each (v1, v2, v3, v4) are relative to the bottom exterior surface of the test cell.

change passing through an expansion valve. Helium boiling occurs continuously and the heater is used to maintain sample well temperatures above the helium boiling point.

The cryostat heater is attached to a copper annulus that is in contact with the bottom radiation baffle. The heat path from the copper block to the test cell is 
through the bottom radiation baffle, sample holder, and flange. Low pressure helium gas introduced into the sample well provides a parallel conduction path between the heater block and the test cell. The annular contact between the copper heater block and the bottom radiation baffle is approximately $1 \mathrm{~mm}$ wide. The lower radiation baffles are spring loaded to allow for the test cell position to be adjusted within the sample well. As a result, the contact resistance between the lower radiation baffle and the copper heater block changes with each test configuration.

Figure 4.1(b) illustrates one of the test cells that has a 10-mm-diameter bore. Four Lakeshore silicon diode DT-670 sensors were used to record temperature at various locations. One sensor (s1) was suspended in the helium exchange gas approximately $1 \mathrm{~cm}$ from the test cell wall. The remaining three sensors (s2-s4) were mounted on the external surface of the test cell. The sensors were secured to the outside of the test cell using 316 SS wire with spring-wire tensioners. The temperatures sensors were connected to a Lakeshore 340 temperature controller. The lower flange mount on the sample holder houses a fifth Si-diode temperature sensor (Scientific Instruments SI410b). The copper heater block contains an NTC RTD X45720 sensor hereby referred to as the 'heater sensor'. The heater and the sample holder sensors were connected to a Lakeshore 331 temperature controller. The heater temperature was set and maintained using an auto-tuned PID control system built into the Lakeshore 331. Gas manifold pressure was logged using a Mensor DPG 15000. The reader is directed to the authors' previous publications for additional details on the experimental setup 


\section{Table 4.1}

Test cells used in the hydrogen and methane experiments and the sensor locations as indicated in figure 4.2. All dimensions are in $\mathrm{mm}$.

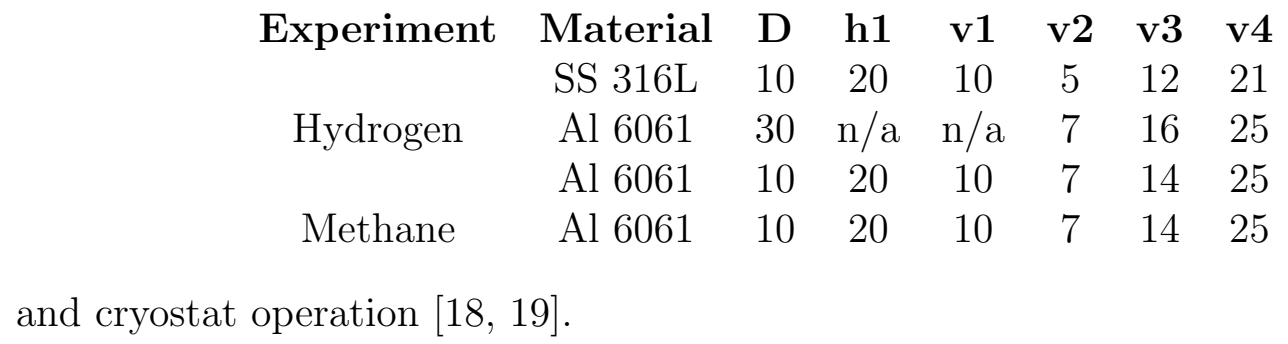

Two experimental campaigns are included in this discussion; tests with hydrogen and with methane. Three different test configurations were used in the hydrogen campaign (Table 4.1). The $10 \mathrm{~mm} \mathrm{Al} 6061$ cell was reused in the methane campaign. Sensor locations on the test cells, listed in Table 4.1, were measured after assembly. Two types of tests were conducted with each test cell. The first was thermal cycling of an evacuated cell referred to as "dry" tests. The second was controlled phase change with cryogenic liquid referred to as "wet" tests.

Dry tests were conducted with a hard vacuum inside the test cell. The cryostat heater was thermally cycled though a range of temperatures and the corresponding temperatures on the test cell, sample holder, heater, and helium gas in the sample well were recorded. Prior to each dry test, the test cell assembly was maintained in thermal equilibrium. Once a steady state temperature was attained, data logging was initiated and the temperature set point on the heater was increased. Temperatures from the four Lakeshore sensors, the sample holder sensor, the heater temperature 


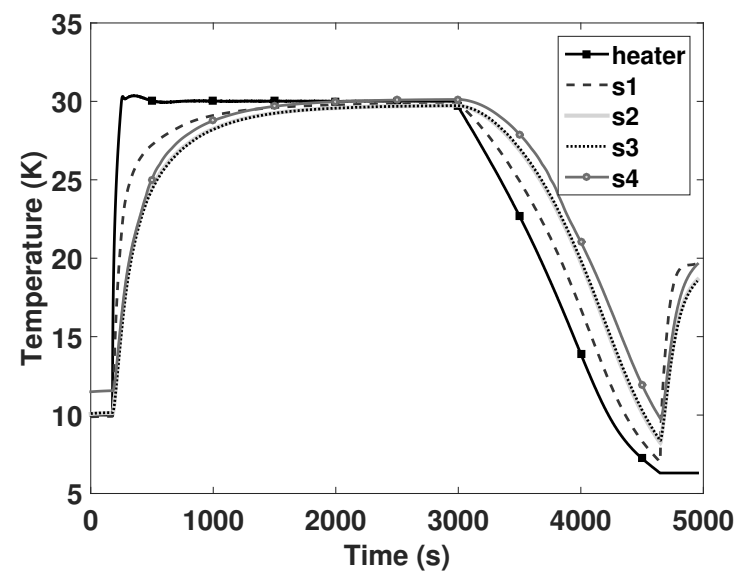

(a) Hydrogen campaign, $10 \mathrm{~mm}$ SS

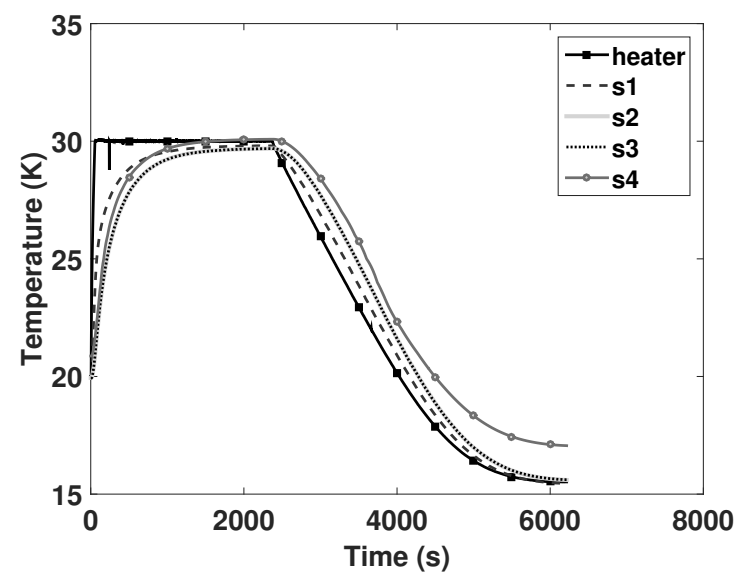

(c) Hydrogen campaign, $10 \mathrm{~mm} \mathrm{Al}$

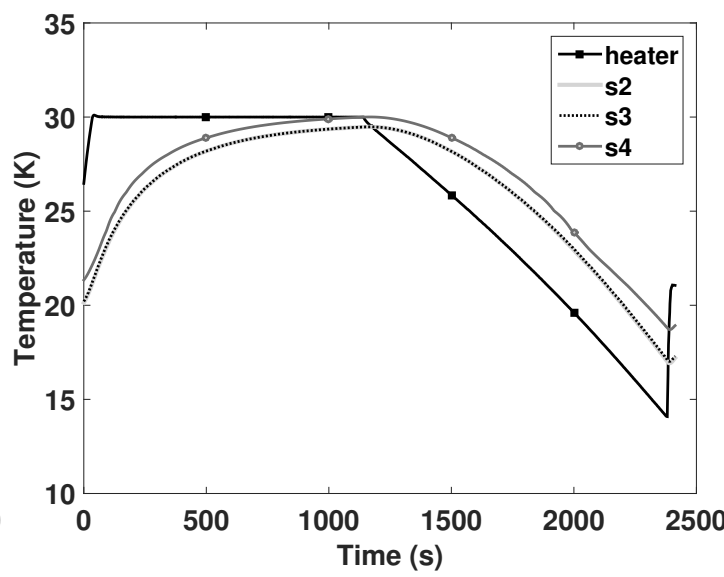

(b) Hydrogen campaign, $30 \mathrm{~mm} \mathrm{Al}$

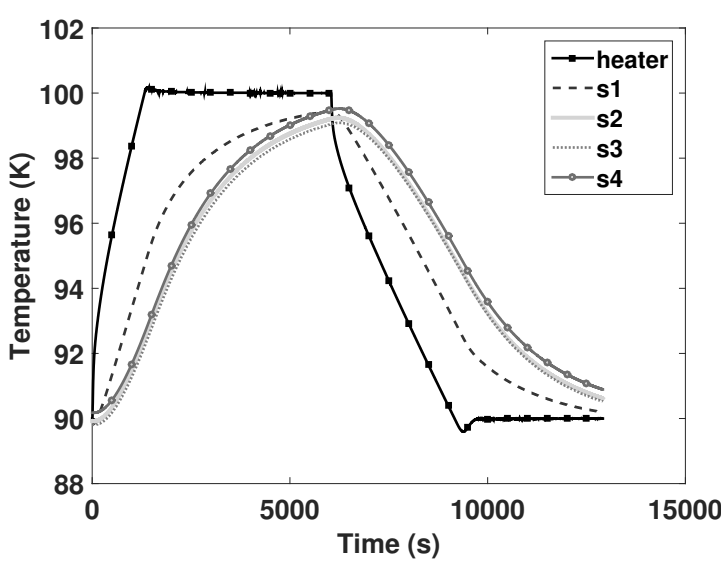

(d) Methane campaign, $10 \mathrm{~mm} \mathrm{Al}$

Figure 4.2: Temperatures during thermal cycling of "dry" cell tests. "Heater" refers to the temperature sensor located in the cryostat heater block.

and the heater power were logged at $1 \mathrm{~Hz}$. After the test cell assembly reached the new equilibrium state, the heater set point was lowered.

The results from thermal cycling of evacuated cells tested in both the hydrogen and methane experiments are shown in figure 4.2. Sensor s1 for the $30 \mathrm{~mm}$ Al cell setup was damaged during the assembly, hence helium temperature data is unavailable for this set of experiments. Dry test experiments in the methane campaign were carried 
about at temperatures between $90 \mathrm{~K}$ - $110 \mathrm{~K}$ (saturation temperature of methane corresponding to $100-210 \mathrm{kPa}$ ). At these temperatures, the thermal transients are longer than those observed in the 20 - $30 \mathrm{~K}$ range during the hydrogen campaign. This delayed response is due to increased resistances to the heat conduction path between the baffle and the test cell as well as significant changes in material properties with temperature.

The source of hydrogen for the wet tests was $99.9995 \%$ pure, water vapor $<5$ ppm, N2 $<2$ ppm, O2 $<1$ ppm, all others undetectable. The methane purity was $99.97 \%$. Vapor was introduced through the feed line in the test cell at a preset pressure controlled by the gas manifold outside the imaging facility. The vapor was condensed inside the test cell by lowering the cryostat temperature below the saturation temperature corresponding to the manifold pressure. After the condensation experiments were complete, the cryostat temperature was increased above the saturation temperature to induce evaporation. During the methane campaign, it was observed that the time to a steady state thermal response upon unit change in temperature was at least 2 orders of magnitude greater than that seen during the hydrogen campaign. The higher temperatures for methane phase change, construction of the cryostat and corresponding changes in material properties result in wait times of several hours while access to the neutron beam was limited to a few days. Hence, in the interest of time, once a steady temperature was attained, it was held constant and the manifold pressure was changed instead to induce condensation and evaporation. 


\subsection{Transient Thermal Modeling of Cryostat}

The objective of the ANSYS/Fluent thermal model is to extract the inner wall temperature distribution from the experimentally measured discrete outer wall temperatures. An axisymmetric model was built using the ANSYS design modeler ${ }^{\top M}$. The computational domain encompassed the sample holder from the bottom radiation baffle to the test cell, the cryostat heater, the sample well and the helium exchange gas. Figure 4.4 is the axisymmetric computational domain with the symmetry axis in the $x$-direction. The components are individually modeled as separate entities so each component could be assigned independent thermal properties and boundary conditions. Shadow walls are used at all component interfaces to transfer heat and model thermal contact.

Data from the evacuated (dry) thermal cycling tests are used to tune the contact resistances in the transient thermal model. Though the temperature change during thermal cycling is modest at 10 to $20 \mathrm{~K}$, values thermal conductivity and specific heat for materials used in the experiments change by an order of magnitude. Temperature dependent conductivity and specific heat for aluminum 6061 [88, 90], copper [90, 117], stainless steel 316 [88, 122] are used in the thermal model. The change in density of solids due to thermal contraction from $300 \mathrm{~K}$ was accounted for using data provided

by [52]. For the helium exchange gas in the sample well, temperature dependent data 


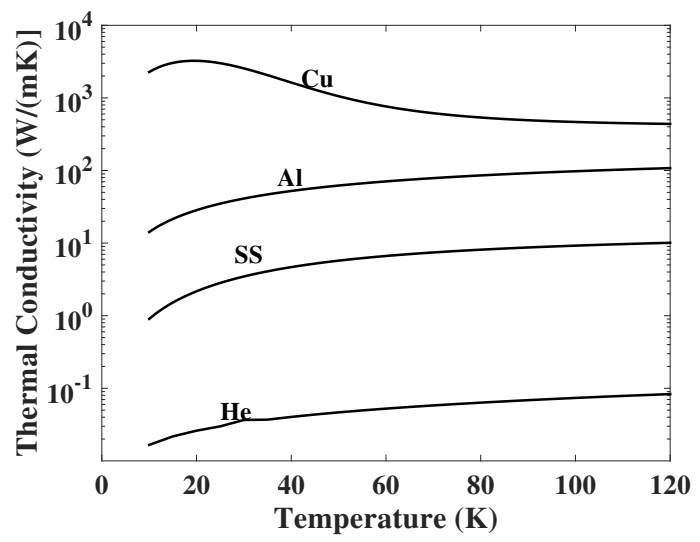

(a) Conductivity

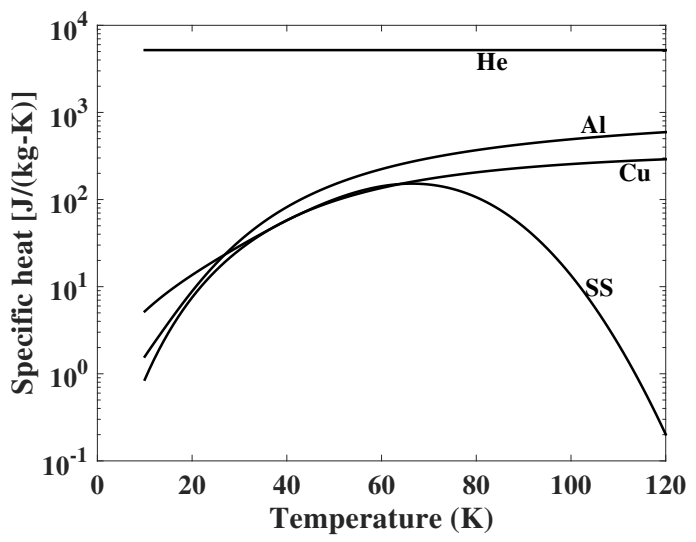

(b) Specific heat

Figure 4.3: Temperature dependent thermal properties

for viscosity, specific heat and thermal conductivity is used [9, 57, 91]. Correlations between thermal conductivity, specific heat and temperature for these materials are shown in figure 4.3. These material properties are tabulated in a lookup table that is read by the ANSYS/Fluent model.

A quad dominant mesh is generated for the domain with size refinement at all contact interfaces. A coarse mesh was initially built and the mesh size in the all the zones was monotonically reduced. The minimum spatial resolution in the test cell outer wall was set to be $50 \mu \mathrm{m}$. Increasing the number of elements beyond 19000 resulted in the relative mean square error of the transient temperature profile $<10^{-10}$ in the the s1 and s2 locations and a maximum relative change less than $100 \mu \mathrm{K}$. The optimal trade-off between accuracy and computational overhead was found with using 19566 elements. The average computational time for a typical dry test is about 8 hours when run in parallel on 16 cores. 


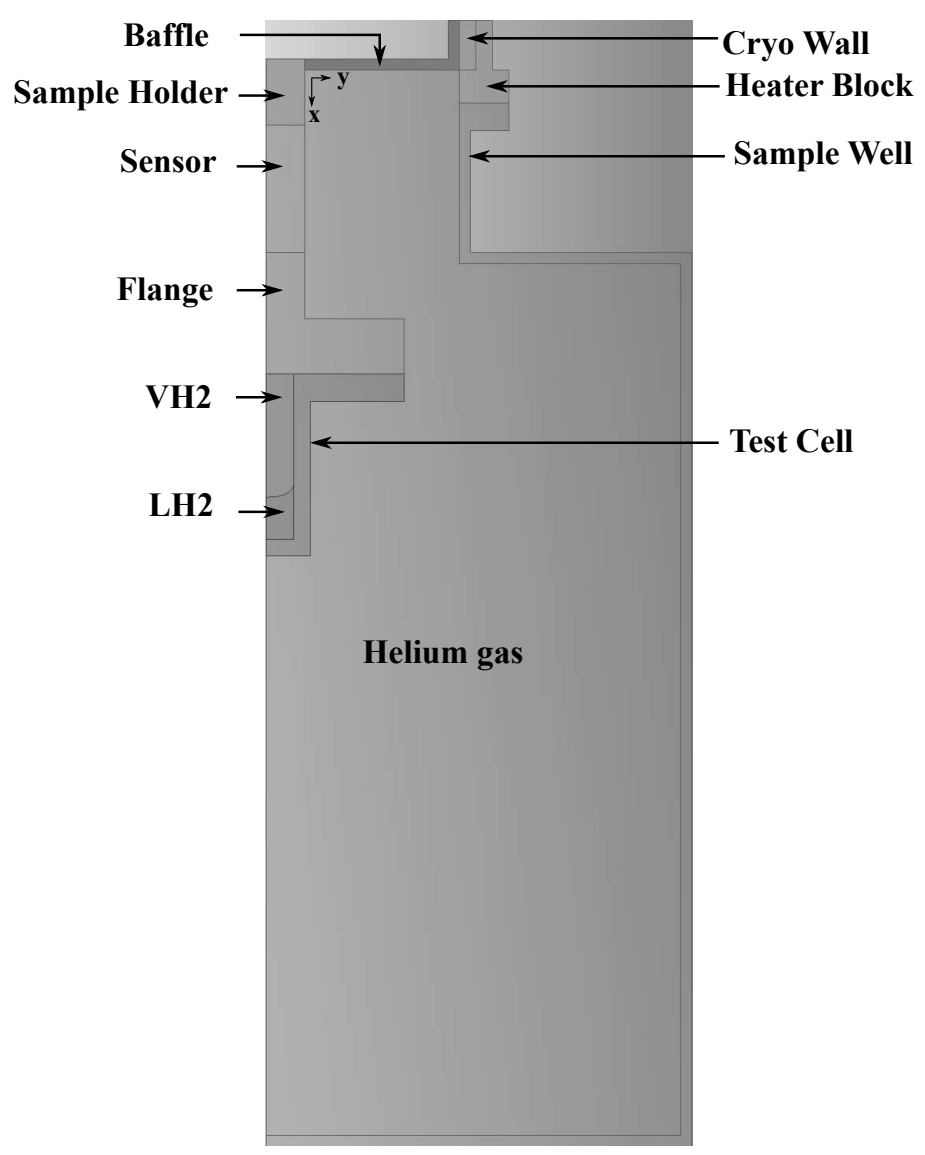

Figure 4.4: Computational domain of the thermal model

The heater temperature logged during the experiments is used as a boundary condition with all outer walls of the domain insulated. For the dry tests, the interior of the test cell is evacuated. Radiation from the sample well to the test cell is less than $5 \%$ of the conductive heat transfer with a perfect vacuum in the sample well. The presence of helium gas would only reduce the amount of heat transfer due to radiation. Therefore, radiation has been neglected in the thermal model. The pressure of the helium exchange gas was approximately $400 \mathrm{~Pa}$. This varied from test-to-test, but not enough to significantly alter the thermal properties of helium in the sample well. 


\subsection{Contact Resistances}

Initial simulations with perfect thermal contact at all the solid-solid interfaces resulted in steady-state predictions approximately four times faster than the experimental values. Six locations were identified as having a significant contact resistance. Five of these resistances are in the conduction path from the heater to the sample holder (stick) and test cell. The sixth resistance is in the conduction path through the helium exchange gas.

Wall-to-Baffle: The bottom radiation baffle of the sample holder is in contact with the heater on a $1 \mathrm{~mm}$ wide annulus with an inside diameter of $68 \mathrm{~mm}$. The baffle is spring loaded to ensure good contact and a direct heat conduction path. A second heat conduction path exists between the baffle and the cryostat wall. The outer circumference of the baffle is $69 \mathrm{~mm}$ and the cryostat has a $70 \mathrm{~mm}$ bore. The gap between the baffle and cryostat wall is filled with helium that is in communication with the sample well. The helium thermal conductivity is 0.01 to $0.03 \mathrm{~W} / \mathrm{mK}$ in the

temperature range of 5 to $20 \mathrm{~K}[57]$. The parallel heat path from the heater-to-baffle and cryostat-to-baffle is modeled as a single contact resistance.

Baffle-to-Sample Holder: The radiation baffle is attached to a spring-loaded sleeve that allows for adjusting the vertical location of the test cell within the sample well. 
The baffle is attached to the sleeve using a cryogenic epoxy (Stycast 2580FT) with a thermal conductivity of $1.3 \mathrm{~W} / \mathrm{mK}$ at room temperature and $0.064 \mathrm{~W} / \mathrm{mK}$ at $4.2 \mathrm{~K}$. Helium, in communication with the sample well, resides in the gap between the baffle sleeve and sample holder. The combined serial resistances of the baffle to epoxy to sleeve to helium to sample holder is modeled as a single contact resistance.

Sample Holder-to-Sensor: The sample holder includes a threaded element that houses a si-diode temperature sensor (Scientific Instruments SI-410b). Data from the sample holder temperature sensor is not used in this analysis because of the large uncertainty with this particular sensor. Helium that is in communication with the sample well resides within the sensor cavity so that there is a parallel heat path along the axis of the sample holder. Heat is conducted through the solid-solid contact and through the solid-helium-solid contact. This is modeled as a single contact resistance.

Sensor-to-Spacer: During the methane campaign, threaded aluminum 6061 spacers were added between the sample holder and test cell flange in order to increase the contact pressure between the heater block and baffle and to provide additional extension of the test cell within the sample well. The conduction path from the sensor housing to spacers (if present) is modeled as a single contact resistance.

Spacer-to-Flange: The flange sealing the the top of the test cell and containing the gas passage is attached to either a spacer or the sensor housing via a threaded connection. The diameter of the threaded contact is $15 \mathrm{~mm}$. The sensor housing and spacers are 
aluminum 6061. The test cell flange (lid) is 316L stainless steel.

Flange-to-Test Cell: The test cell is bolted to the flange using six 4-40 aluminum bolts and sealed against leakage using an indium seal. Figure 4.1(b) illustrates the test cell to flange geometry for the 10-mm-diameter test cells. The contact area between the test cell and flange varied with each test cell.

Heater-to-Sample Well: The sample well is bolted to the copper heater block and provides a secondary heat conduction path from the sample well wall through the helium exchange gas to the test cell. The contact between the heater block and the sample well is modeled as a contact resistance.

Thermal contact resistances are modeled using the thin wall conduction model in FLUENT, which solves a 1D conduction equation between the two shadow walls that define the interface. The thin wall conduction model requires specification of a conductivity for an imaginary material of interface and a wall thickness. Initially all contact interfaces are set to a thickness of $1 \mathrm{~mm}$ and a conductivity of $0.1 \mathrm{~W} / \mathrm{mK}$. An iterative methodology is used to adjust the shadow wall thickness and thermal conductivity for the six contact resistances. 


\subsection{Determining Contact Resistances from Dry Cell Thermal Cycling}

The transient axisymmetric model is evaluated using ANSYS FLUENT to solve the equations of continuity, momentum and energy. A pressure based approach is employed and the pressure velocity coupling is achieved using the Corrected Semi Implicit Pressure Linked Equation with a zero skewness factor. The pressure, density, momentum and energy is evaluated using the body force weighted method, second order upwind, second order upwind and third order MUSCL methods respectively. A first order implicit time stepping method was used for temporal resolution. A time step of $1 \mathrm{~s}$ was deemed to suffice since the time to steady state is on the order of 1000 s. The default under relaxation parameters were used in the simulation and

the residuals were set to $10^{-2}$ for continuity, $10^{-6}$ for $x$ and $y$ velocity components and $10^{-16}$ for the energy equation. Approximately 50 iterations are necessary for convergence at each time step. The convergence criteria was set at a minimum of 3 order reduction in the residuals of continuity, velocities and energy.

A manual optimization method is used to determine a set of resistances that accurately captured the transient temperatures in both the helium gas (s1) the exterior test cell surface (s2-s4). The Wall-to-Baffle resistance was set to that of a $1 \mathrm{~mm}$ wide 
annulus filled with helium gas at the heater temperature. The conjugate heat transfer encountered here allows for the heat to propagate through a parallel path (helium gas) if the resistance in the primary path (conduction through the baffle-sample holder) is high. The highest resistance is at the baffle-sample holder interface due to the sleeve and the cryogenic epoxy. Hence, the Baffle-to-Sample Holder resistance was first increased to minimize the difference in time constants between the simulation and experiments. The time constants could be matched to within 30\%, but further increase in resistance beyond a certain value had no effect on the time constant. The Wall-to-Baffle resistance was fixed at the value that resulted in less than $1 \%$ relative change of the time constant. Then, the Sample Holder-to-Sensor and the Sensor-toFlange resistances were increased equally until the relative time constant change was less than 1\%. At this stage, the time constants could be matched to within $10 \%$. The remaining two resistances: Flange-to-Test Cell and Heater-to-Sample Well are determined through a parametric least squares routine by comparing the simulation results to the experimental helium gas temperature (s1) and the outer wall temperature (s2). The process is terminated when the simulated time constant is within $1 \%$ of the experimental data and the simulation temperatures are within the sensor uncertainty $( \pm 0.25 \mathrm{~K})$. The error in the heater temperature was $\pm 0.1 \mathrm{~K}$. As a result, the relative uncertainty in the reported resistance parameters are $<1.3 \%$. The contact resistances for the hydrogen and methane campaigns are listed in Tables 4.2 and 4.3, respectively. 
Table 4.2

Contact resistances determined from the hydrogen experiments. Units for contact area are $\mathrm{mm}^{2}$, conductivities are $\mathrm{W} / \mathrm{mK}$, and resistances are $\mathrm{K} / \mathrm{W}$.

\begin{tabular}{clllllll}
$\begin{array}{l}\text { Contact } \\
\text { Interface }\end{array}$ & $\begin{array}{l}\text { Wall - } \\
\text { Baffle }\end{array}$ & $\begin{array}{l}\text { Baffle } \\
\text { Sample } \\
\text { Holder }\end{array}$ & $\begin{array}{l}\text { Sample } \\
\text { Holder } \\
\text { Sensor }\end{array}$ & $\begin{array}{l}\text { Sensor } \\
\text { Flange }\end{array}$ & $\begin{array}{l}\text { Flange - } \\
\text { Testcell }\end{array}$ & $\begin{array}{l}\text { Heater - } \\
\text { Sample } \\
\text { Well }\end{array}$ \\
\hline & Contact area & 4398.230 & 87.965 & 153.938 & 153.938 & 1178.097 & 2233.672 \\
\hline $10 \mathrm{~mm} \mathrm{SS}$ & Conductivity & 0.020 & 0.020 & 0.100 & 0.100 & 0.300 & 0.300 \\
$(20 \mathrm{~K}-30 \mathrm{~K})$ & Resistance & 11.368 & 852.616 & 64.961 & 64.961 & 2.829 & 1.492 \\
\hline $30 \mathrm{~mm} \mathrm{Al}$ & Conductivity & 0.025 & 0.025 & 0.100 & 0.100 & 0.350 & 0.300 \\
$(20 \mathrm{~K}-30 \mathrm{~K})$ & Resistance & 10.004 & 727.565 & 64.961 & 64.961 & 2.425 & 1.492 \\
\hline $10 \mathrm{~mm} \mathrm{Al}$ & Conductivity & 0.025 & 0.025 & 0.100 & 0.100 & 0.250 & 0.250 \\
$(15 \mathrm{~K}-30 \mathrm{~K})$ & Resistance & 9.095 & 682.093 & 64.961 & 64.961 & 3.395 & 1.791
\end{tabular}

Table 4.3

Contact resistances determined from the methane experiments with the 10 $\mathrm{mm} \mathrm{Al}$ cell. Units for contact area are $\mathrm{mm}^{2}$, conductivities are $\mathrm{W} / \mathrm{mK}$, and resistances are $\mathrm{K} / \mathrm{W}$.

$\begin{array}{llllllll}\begin{array}{l}\text { Contact In- } \\ \text { terface }\end{array} & \begin{array}{l}\text { Wall - } \\ \text { Baffle }\end{array} & \begin{array}{l}\text { Baffle } \\ \text { Sample } \\ \text { Holder }\end{array} & \begin{array}{l}\text { Sample } \\ \text { Holder - } \\ \text { Sensor }\end{array} & \begin{array}{l}\text { Sensor } \\ \text { Spacer }\end{array} & \begin{array}{l}\text { Spacer } \\ \text { Flange }\end{array} & \begin{array}{l}\text { Flange - } \\ \text { Testcell }\end{array} & \begin{array}{l}\text { Heater - } \\ \text { Sample } \\ \text { Well }\end{array} \\ \text { Contact Area } & 4398.23 & 87.96 & 153.94 & 153.94 & 153.94 & 1178.10 & 2233.67 \\ \text { Conductivity } & 0.07 & 0.07 & 0.10 & 0.10 & 0.10 & 0.40 & 0.40 \\ \text { Resistance } & \mathbf{3 . 2 5} & \mathbf{2 4 3 . 6 0} & \mathbf{6 4 . 9 6} & \mathbf{6 4 . 9 6} & \mathbf{6 4 . 9 6} & \mathbf{2 . 1 2} & \mathbf{1 . 1 2}\end{array}$

In the methane simulations, the Sensor-to-Spacer and Spacer-to-Flange resistances are changed in lieu of the Sensor-to-Flange resistance. This procedure provides one of several possible sets of solutions that results in accurate tracking of transient temperatures. To obtain a unique solution, experimental temperatures at several points in the conduction path (for example, baffle, flange, sample holder, etc) are needed.

Figures 4.5 and 4.6 compare the simulation results to the experimentally measured temperatures from the hydrogen dry cell experiments. Figure $4.5(\mathrm{a})$ is of sensor s1 on the 10-mm SS 316 test cell and the 10-mm AL 6061 test cell. Figure 4.5(b) is the same comparison shown as a log-temperature difference, in which the accuracy 


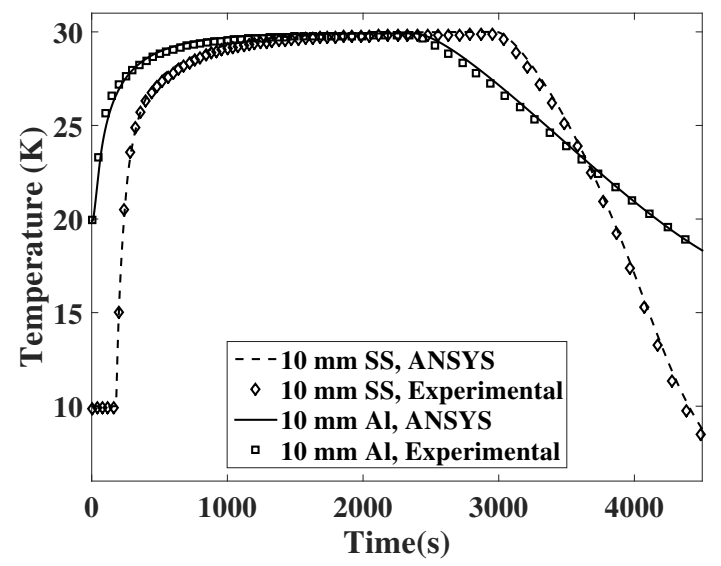

(a) Sensor s1

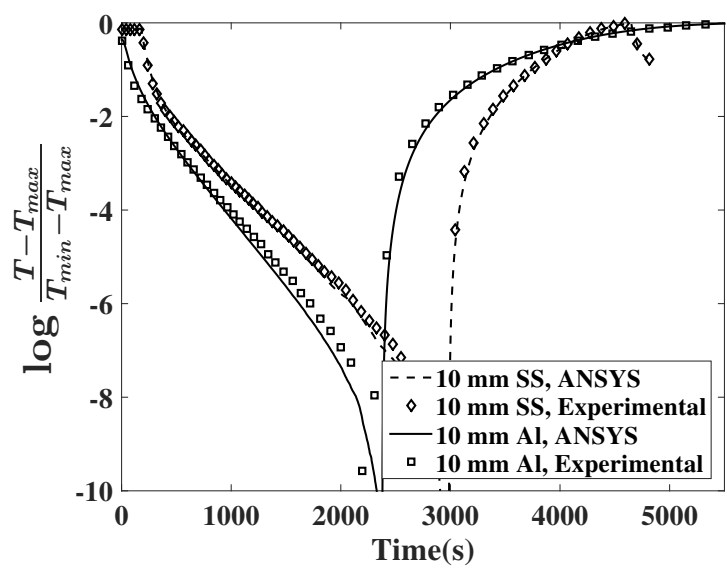

(b) Sensor s1, log-temperature difference

Figure 4.5: Comparison of simulation and hydrogen experiments for sensor s1.

of the model to capture the time response of the cryostat assembly is evident. Figures 4.6(a) and 4.6(b) are the same comparison for sensor s2 with the addition of the 30-mm AL 6061 test cell. Results for sensor s3 are not shown because these are identical to sensor s2. Figures 4.7(a) and 4.7(b) compare the simulation results to the experimentally measured temperatures from the methane dry cell experiments. The marker size in figures 4.5, 4.6, and 4.7 is equivalent to the measurement uncertainty at the corresponding temperature.

Variation in the exchange gas pressure had little effect on the transient response. Helium exchange gas pressure was varied in the model from $10 \mathrm{~Pa}$ up to $1 \mathrm{kPa}$ and the resulting transient temperatures at the s2 location had a relative mean square error less than $10^{-10}$ and a maximum relative change less than $100 \mu \mathrm{K}$. Maximum helium velocities in the simulations are on the order of $10^{-3} \mathrm{~m} / \mathrm{s}$ with corresponding Rayleigh number (non-dimensional number that represents the ratio of buoyant forces 


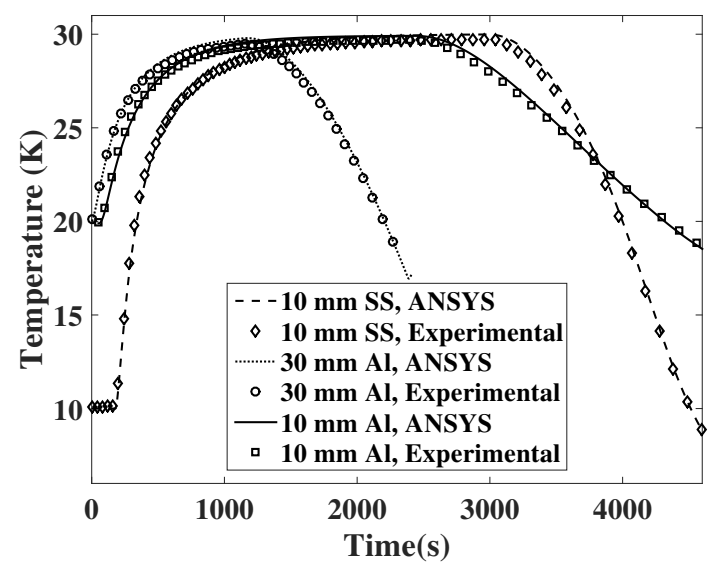

(a) Sensor s2

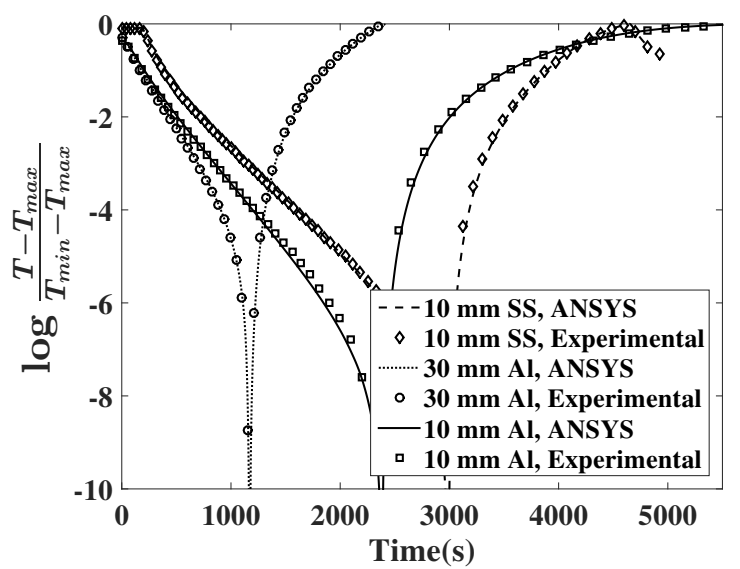

(b) Sensor s2, log-temperature difference

Figure 4.6: Comparison of simulation and hydrogen experiments for sensor s2.

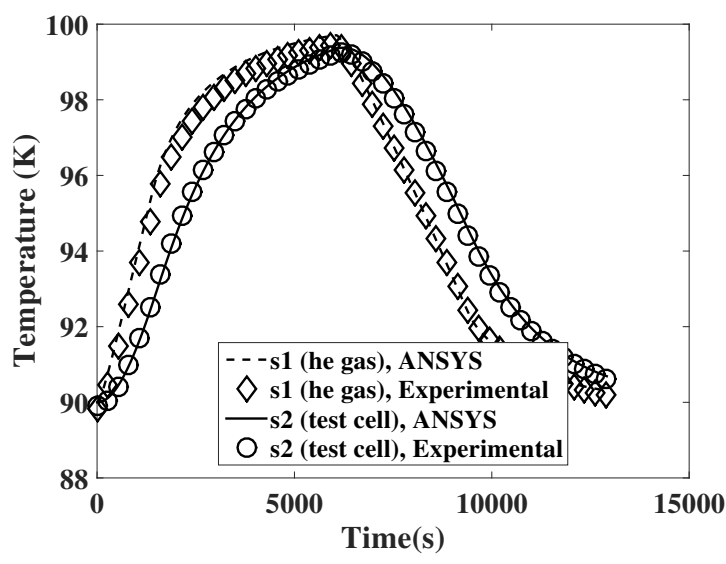

(a) Sensors s1 and s2

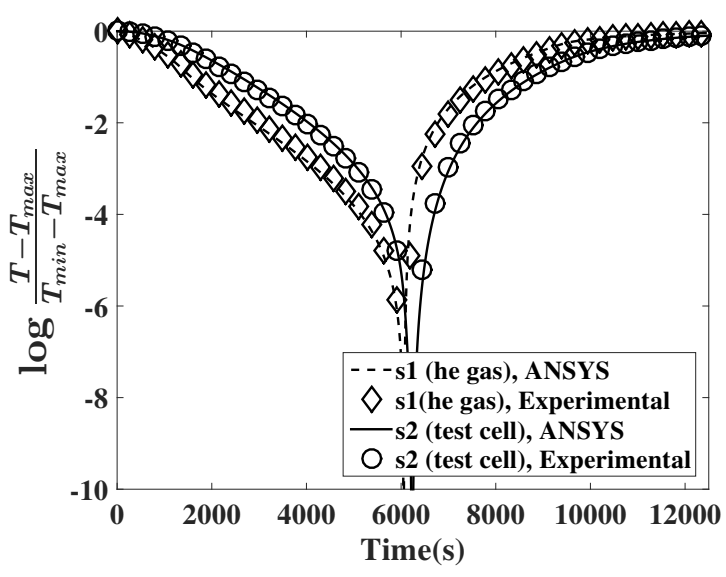

(b) Sensors s1 and s2, log-temperature difference

Figure 4.7: Comparison of simulation and methane experiments for sensors s1 and s2.

to viscous forces) less than 100. In order to test the role of convection in the sample well, the helium was modeled as a solid with a constant density and temperature dependent properties of helium gas. These simulations yielded similar results as the convective cases. Variation in temperatures between fluid and solid simulations are less than $100 \mu \mathrm{K}$. Modeling the helium exchange gas as a solid decreases the 
computational time of the simulations by $15 \%$.

\subsection{Thermal Modeling for Phase Change Experi-}

\section{ments}

Once the dry cell thermal cycling tests were completed, hydrogen or methane was condensed in the test cell to begin the phase change (wet) tests. The hardware configuration remained the same. As such, the contact resistances determined from the dry cell thermal cycling data remain valid for the wet tests. Figure 4.8 illustrates the boundary conditions for the computational domain inside the test cell. On the vapor side of the test cell domain, a uniform mass flux boundary condition and a constant pressure condition corresponding to saturation at the test cell exit are imposed. Contact resistances determined from the dry cell thermal cycling data are used and the heater temperature serves as a thermal boundary condition for heat flux to the test cell.

For all hydrogen tests, the measured evaporation rates for each run were between 0.4 to $0.6 \mathrm{mg} / \mathrm{s}$, corresponding to a bulk meniscus velocity of 14 to $25 \mu \mathrm{m} / \mathrm{s}$, which remained constant throughout the evaporation test. The evaporation rate is slow with

a Peclet number less than $10^{-2}$, indicating that the dominant mode of heat transfer to 
the test cell during phase change is conduction. Because the liquid-vapor in the test cell is near thermal equilibrium, the liquid domain is treated as a solid with temperature dependent properties corresponding to liquid hydrogen [86, 92]. Thermodynamic properties of both liquid and vapor are determined using the fundamental equation of state described by [83].

Simulation of liquid hydrogen evaporation presented here correspond to the $10 \mathrm{~mm} \mathrm{Al}$ test cell. During evaporation testing the manifold pressure corresponds to a saturation temperature of $21 \mathrm{~K}$ while the heater was set to $23 \mathrm{~K}$. The liquid-vapor interface profile and location is then imported into ANSYS design modeler ${ }^{\mathrm{TM}}$ and a cubic spline fit is performed. Meshing the interface as extracted from the images proved to be a challenge especially when the film thickness becomes less than $1 \mu \mathrm{m}$. In order to avoid a highly skewed mesh at the three phase contact point, the liquid film was terminated at $10 \mu \mathrm{m}$ as shown in figure 4.8. Computational expense increased by two orders of magnitude for each order of magnitude reduction in film thickness less than $10 \mu \mathrm{m}$. The mesh for all regions outside the test cell was the same as in the dry model. Liquid and vapor regions inside the cell have a quad-dominant mesh with nominal element size of $100 \mu \mathrm{m}$ in the bulk region gradually reducing to $10 \mu \mathrm{m}$ near all interfaces (solid-liquid, liquid-gas and gas-solid). Further reduction in the mesh size results an relative mean square error $<10^{-10}$ in the inner wall temperature and a maximum relative change $<100 \mu \mathrm{K}$. 
Experimentally measured meniscus profiles did not exhibit any hysteresis during recession and the meniscus shape remained the same during evaporation and condensation tests [18, 19]. Therefore, phase change is modeled as quasi-steady with a fixed interface location. Heat loss at the wall due to evaporation was modeled using a uniform heat-sink on the liquid side of the liquid-vapor interface. The rate of energy and mass exchange during evaporation is determined from the experimentally measured rate of meniscus recession within the test cell. Although the magnitude of the total heat loss is known from macroscale observations, the appropriate location of the heat sink in the thermal model is not. Thin film evaporation modeling suggests that a majority of the evaporative flux occurs in the contact line region [43, 47, 53, 65, 106, 108, 127]. For these simulations the evaporative flux is uniformly distributed along a $0.95 \mathrm{~mm}$ section of the the contact line region beginning at the $10 \mu \mathrm{m}$ termination point. For a length over which the evaporative flux is distributed between 0.90 to $1.2 \mathrm{~mm}$, the thermal model results do not change. If the length is increased beyond $1.2 \mathrm{~mm}$ the prediction of the temperature at sensor s1, located in the helium space, begins to deviate from experimental values.

The experimental inputs to the model are the temperature of the heater during evaporation, manifold pressure, and rate of evaporation. The heater temperature is held constant at $23^{\circ} \mathrm{K}$. The manifold pressure is held constant at $121.5 \mathrm{kPa}$ (abs), which corresponds to a saturation temperature of $21^{\circ} \mathrm{K}$. And the evaporative mass rate is 


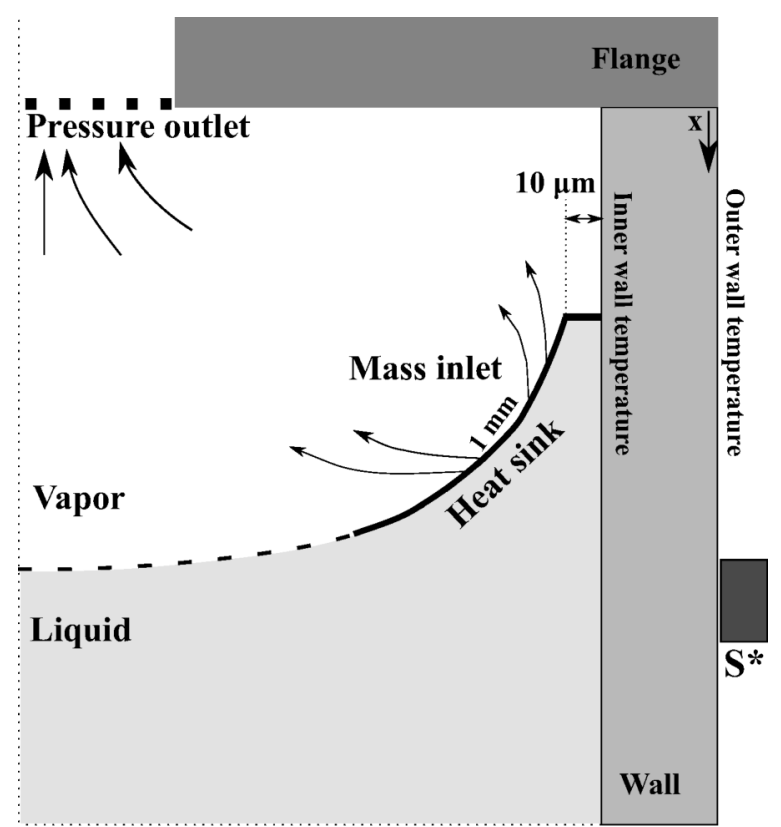

Figure 4.8: Boundary conditions for vapor domain and liquid-vapor interface.

$0.556 \mathrm{mg} / \mathrm{s}$. The steady-state temperature contours for the entire computational domain and velocity streamlines in the vapor domain are shown in figure 4.9. Within the test cell, liquid, vapor and solid temperatures temperatures are within $0.1 \mathrm{~K}$ of saturation. Temperature variations between the liquid, vapor and/or test cell wall is lower than resolution of the Si diode temperature sensors used but within the resolution of the thermal model. The maximum velocity in the hydrogen vapor is less than $0.02 \mathrm{~m} / \mathrm{s}$ corresponding to a Reynolds number below 300 .

The inner and outer wall temperature distributions are shown in figure 4.10. The distance is relative to the bottom of the test cell as shown in figure $4.1(\mathrm{~b})$. The $10 \mu \mathrm{m}$ thin film termination is located at $10 \mathrm{~mm}$. Outer wall temperatures are within the measured temperature uncertainty for sensors s1-s3 as shown in figure 4.10, where 
s1 is located in the helium exchange gas. Experimental readings from sensor s4 are approximately $0.5 \mathrm{~K}$ above that predicted by the thermal model. This difference is due to a temperature dependent thermal offset in the calibration of sensor s4, which can also be observed in the dry cell thermal cycling data in figure 4.2. Sensor s4 consistently indicates a $0.5 \mathrm{~K}$ higher temperature as compared to the other 3 sensors even under equilibrium conditions. When corrected for this bias, the predicted temperature at the s4 location also lies within the sensor uncertainty. The variation in the inner wall temperatures due to the uncertainty in resistance values are $<10^{-3} \mathrm{~K}$.

The inner wall distribution, shown in figure 4.10, exhibits a minimum temperature in the contact line region. From the contact line region towards the heater, the temperature increases linearly as expected,indicating steady conduction. From the contact line region towards the bottom of the test cell, the temperature also increases, though not strictly linearly. The temperature variation in the contact line region is small in magnitude, approximately $10^{-2} \mathrm{~K}$, but the distance over which this variation occurs is also small, approximately $50 \mu \mathrm{m}$. As a result, the temperature gradient in the contact line region is on the order of $10^{3} \mathrm{~K} / \mathrm{m}$, which is significant considering the liquid film thickness at this location is on the order of 0.1 to $1 \mu \mathrm{m}$.

At these thicknesses the temperature gradient along the liquid-vapor interface will mirror that along the solid-liquid interface (wall) because of the short heat conduction path. Significant thermocapillary stresses will occur even in a pure liquid-vapor 
system due to the localization of evaporative flux. While soluto-capillary interface stresses have been predicted for evaporation of binary mixtures [127], thermocapillary interface stresses are not thought to occur in single-component liquid-vapor phase change because localized evaporation and condensation should equilibrate any local temperature variation on the interface. Modeling results of thin liquid films tend to validate or presume this assumption, but these same models impose a constant wall temperature condition [43, 53, 65, 106, 127]. The sustained non-uniformity of the wall temperature distribution as shown in this thermal model has the potential to maintain thermocapillary stresses on the liquid-vapor interface even in pure liquid-vapor systems in the contact line region. For this temperature gradient, thermocapillary stresses will tend to suppress capillary flow into the contact line region; thereby decreasing the overall evaporation rate.

\subsection{Summary and Conclusion}

In summary, a thermal model of heat transfer of a cryostat environment has been developed that accurately captures steady state and transient temperatures. Accurate modeling is complicated by the number of contact resistances that can change between tests and the temperature dependency of material properties. The contact resistances were determined through an iterative method comparing predicted temperatures with measured temperatures on the exterior of an evacuated test cell undergoing thermal 


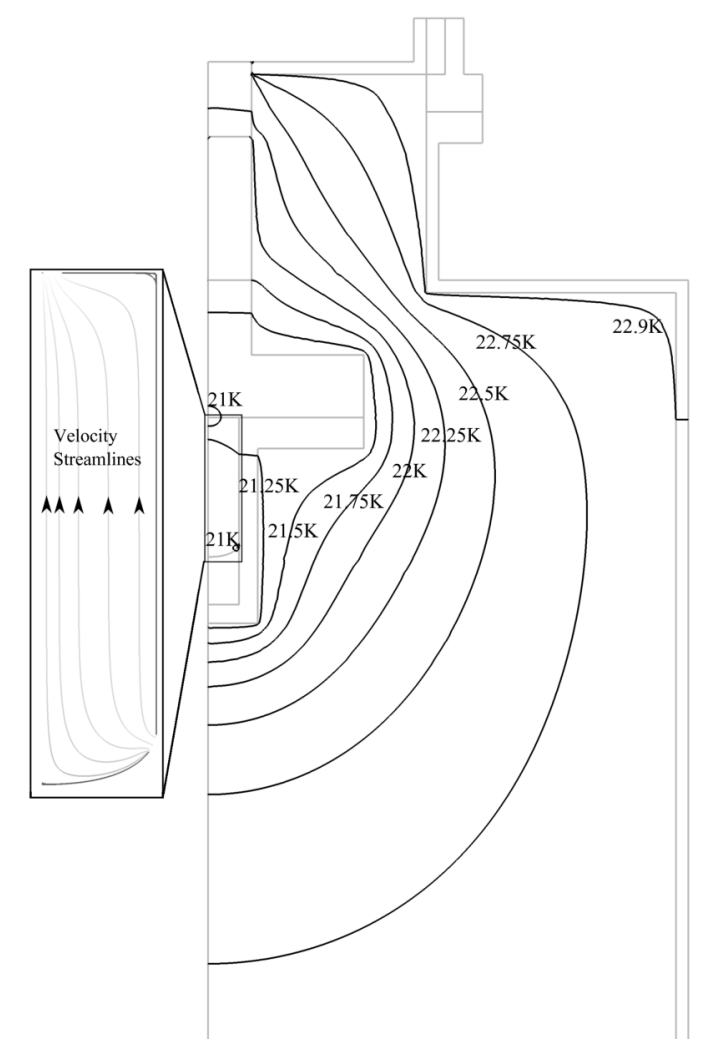

Figure 4.9: Temperature contour in the sample well and velocity streamlines in the vapor space of the test cell during steady state phase change of liquid hydrogen saturated at $21 \mathrm{~K}$. The heater was set at $23 \mathrm{~K}$. Streamlines emanate from the contact region and terminate at the exit of the test cell. Vapor velocities are less than $2 \mathrm{~cm} / \mathrm{s}$.

cycling. The purpose of the thermal model is to two-fold. Firstly, it is to determine the temperature distribution on the interior of the test cell. Secondly, it is to establish an accurate prediction of the rate of conduction heat transfer along the sample holder and through the helium exchange gas during condensation/evaporation of liquid hydrogen or liquid methane.

The thermal model was first used to determine the effective contact resistances for each test configuration, which covered two temperature ranges (hydrogen and 


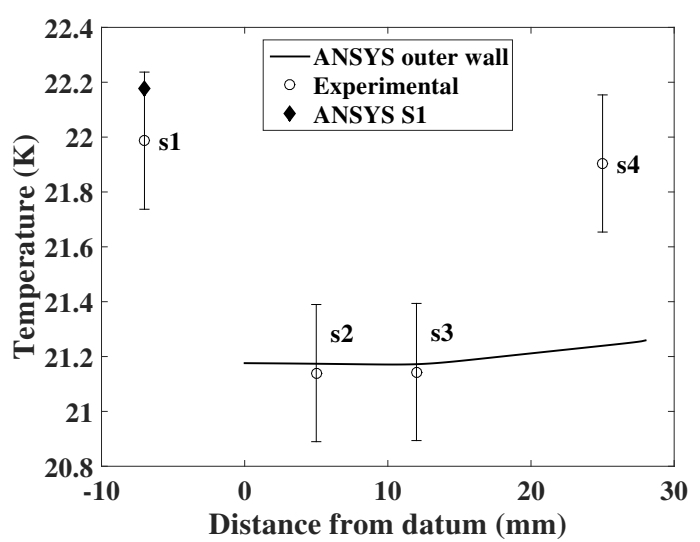

(a) Exterior Surface of Test Cell

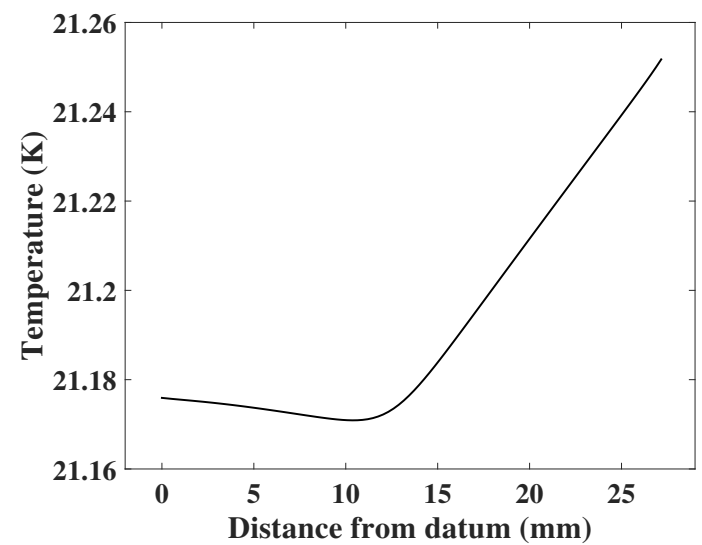

(b) Interior Surface of Test Cell

Figure 4.10: Temperature distributions along the exterior and interior of the test cell. The datum is located at the bottom of the test cell as shown in figure 4.1. Sensor s4 deviation due to $0.5 \mathrm{~K}$ bias error in the measurement. The contact line is located at $10 \mathrm{~mm}$.

methane) and four test cell configurations. Then the thermal model was applied to hydrogen evaporation at $21 \mathrm{~K}$ in the $10-\mathrm{mm}$ diameter $\mathrm{Al}$ test cell to predict inner and outer wall temperature distributions. The outer wall temperature predictions match the experimentally measured values during this test. The predicted temperature gradient between the contact line location and the flange provides the rate of heat conduction from the heater. The thermal model also provides the rate of heat conduction through the helium exchange gas on the liquid side of the test cell. The inner wall temperature distribution exhibits a large gradient in the contact line region, which will likely result in unanticipated thermocapillary stresses during evaporation. Predictions by this thermal model will enable relaxation of the constant wall temperature boundary condition. The more accurate thermal boundary conditions will allow for deeper investigations into the kinetic model and the underlying physics of phase change. 


\section{Chapter 5}

\section{A combined experimental and}

\section{multi-scale modeling approach to}

determine accommodation

\section{coefficients of cryogenic propellants}

Kinetic theory of phase change is an essential tool to develop reliable models for predicting boil-off and cryo-storage stability for liquid propellants in low gravity. The current state of knowledge on evaporation/condensation processes is insufficient for designing large cryogenic depots critical to the long-term space exploration missions

[41]. The ability to predict the rate of phase change inside propellant tanks remains 
a challenge primarily due to the absence of evaporation-condensation coefficients and the inability to computationally capture the local thermodynamics [3, 4, 18, 19, 60, 99,

101]. In addition to space technology, accurate modeling of phase change is essential in atmospheric science and climate [76, 111], aerosol transport [95, 103] micro- and nano-scale thermal transport in MEMS applications [16, 34, 118].

\subsection{Kinetic Model of Phase Change}

Classical kinetic theory is a statistical description of the behavior of gases based on velocities of the constituent molecules. Kinetic theory has provided the basis for modeling phase change in cases where the mass transport across the interface is not limited by the diffusion in the vapor phase.

Under equilibrium conditions, the vapor in the vicinity of the liquid-vapor interface can be approximated as a perfect (ideal) gas and the velocity distribution of the vapor molecules follows a Maxwell Boltzmann distribution [37]. The reader is directed to Appendix A of Carey [35] for a brief introduction to the fundamentals of kinetic theory and the derivation of the Maxwell Boltzmann distribution. This velocity distribution leads to an expression for the maximum frequency of collision of the vapor molecules on the liquid-vapor interface and was first developed by Hertz

64]. However, the process is dynamic and undergoes simultaneous evaporation and 
condensation. Hence, a net phase change flux can be defined as an algebraic sum of evaporation and condensation fluxes. The mass flux relationship developed by Hertz

64] was found to be inconsistent with many experimental studies [51, 89]. Knudsen measured the evaporation rate of mercury and found that the experimentally measured rate was always lower then the theoretical maximum described by Hertz. He introduced evaporation and condensation coefficients to account for the deviation from the theoretical maximum rate [75].

$$
\dot{m}^{\prime \prime}=\sqrt{\frac{m}{2 \pi k_{b}}}\left(\alpha_{e} \frac{P_{l i}}{\sqrt{T_{i}}}-\alpha_{c} \frac{P_{v i}}{\sqrt{T_{v}}}\right)
$$

where $\dot{m}^{\prime \prime}$ is the mass flux, $\alpha_{e}$ is the evaporation coefficient, $\alpha_{c}$ is the condensation coefficient, $m$ is the mass of the molecule, $k_{b}$ is the Boltzmann's constant, $P_{l i}$ is the liquid pressure at the interface, $P_{v i}$ is the vapor pressure at the interface, $T_{i}$ is the temperature of the liquid at the interface and $T_{v}$ is the vapor temperature.

When a vapor molecule is incident on the interface, it can interact in 3 ways: (1) the molecule can condense (i.e., the vapor molecule is absorbed into the bulk of the liquid), (2) the molecule can be reflected back into the vapor space or (3) the molecule can displace a liquid molecule thereby undergoing a simultaneous evaporation, condensation process. The definitions of the evaporation/condensation coefficients vary slightly between researchers but the general consensus is that the condensation coefficient $\left(\alpha_{c}\right)$ is defined to be the ratio of molecules that undergo condensation to 
the number of molecules that are incident on the surface [12, 51, 89]. Therefore the magnitude must be between 0 and 1 . The evaporation coefficient $\left(\alpha_{e}\right)$ can be defined similarly from the liquid perspective. These coefficients must be determined empirically [1].

The most widely used kinetic approach to model phase change today was initially developed by Schrage [116] in 1953. Schrage argued that during steady phase change there is a net macroscopic velocity of the vapor molecules either towards or away from the interface. This is also referred to as a "drift" velocity. He superimposed the drift velocity with the Maxwell Boltzmann distribution to develop a correction factor $(\mathcal{T})$. Schrage's modified formulation can be expressed by,

$$
\dot{m}^{\prime \prime}=\sqrt{\frac{m}{2 \pi k_{b}}}\left(\alpha_{e} \frac{P_{l i}}{\sqrt{T_{i}}}-\mathcal{T}(a) \alpha_{c} \frac{P_{v i}}{\sqrt{T_{v}}}\right)
$$

where, $a$ is the ratio of the drift velocity to the mean thermal velocity of the vapor molecules (equation 5.2b) and $\mathcal{T}$ is the correction factor expressed by equation 5.2c.

$$
a=\frac{w_{0}}{\sqrt{2 k_{b} T_{v} / m}}
$$




$$
\mathcal{T}(a)=\exp \left(a^{2}\right)+a \sqrt{\pi}[1+\operatorname{erf}(a)]
$$

$w_{0}$ is the drift velocity in equation $5.2 \mathrm{~b}$ and is given by $w_{0}=\dot{m}^{\prime \prime} / \rho_{v}$, where $\rho_{v}$ is the vapor density. In equilibrium, the evaporation flux is equal and opposite to the condensation flux. The Maxwellian assumption is strictly applicable only in equilibrium [116]. However, it is common to use a Maxwellian distribution with a negligible drift velocity to model liquid-vapor phase change [12, 35, 85]. If the drift velocity is small in comparison to the thermal velocity, equation $5.2 \mathrm{C}$ reduces to $\mathcal{T}(a) \approx 1+a \sqrt{\pi}[35]$. If the ideal gas expression is used to evaluate $\rho_{v}$, then, in the limit of small $a$, the original Schrage expression (equation 5.2a) can be reduced to equation $5.2 \mathrm{~d}[12,35,85]$.

$$
\dot{m}^{\prime \prime}=\frac{2}{2-\alpha_{c}} \sqrt{\frac{m}{2 \pi k_{b}}}\left(\alpha_{e} \frac{P_{l i}}{\sqrt{T_{i}}}-\alpha_{c} \frac{P_{v i}}{\sqrt{T_{v}}}\right)
$$

In the Hertz-Knudsen expression (equation [5.1) and simplified Schrage expression (equation $5.2 \mathrm{~d})$, if $\alpha_{c}=\alpha_{e}=\alpha$, then the kinetic factor in the Hertz-Knudsen expression 1 (equation 5.1) is simply $\alpha$ while in the Schrage expression (equation $5.2 \mathrm{~d}$ it is $2 \alpha /(2-\alpha)$. If $\alpha$ is assumed equal to 1 , then the simplified Schrage expression

\footnotetext{
${ }^{1} \mathrm{~A}$ majority of papers erroneously refer to equation $5.2 \mathrm{~d}$ as the original Schrage expression. Although it is derived from the original equation developed by Schrage [116], there are two inherent assumptions: (1) the drift velocity of the vapor molecules is small in comparison to the mean thermal velocity (2) ideal gas equation used to evaluate vapor density.
} 
predicts twice the mass flux predicted by the Hertz-Knudsen expression.

The modified Schrage expression is the most common form of the kinetic phase change model used. The primary complication in evaluating this kinetic theory expression (equation $5.2 \mathrm{~d}$ ) is that the interfacial temperature $\left(T_{i}\right)$ and both kinetic coefficients are unknown. Even if $T_{i}$ is measured or approximated, there remain two unknowns, $\alpha_{c}$ and $\alpha_{e}$, in the expression for $\dot{m}^{\prime \prime}$. For sake of closure it is common practice to assume that the condensation coefficient is equal to the evaporation coefficient $\left(\alpha_{c}=\alpha_{e}=\alpha\right)$ [11, 43, 44, 66, 89, 95, 106, 123 125]. Under this assumption the only remaining coefficient in equations 5.1 and $5.2 \mathrm{~d}$ is $\alpha$, which is referred to as the accommodation coefficient. Several researchers further assume $\alpha=1$ in their models even though this is just the theoretical upper limit and a majority of reported values for $\alpha$ are one to two orders of magnitude less than unity [89].

\subsection{Interface Curvature}

Experiments with cryogens in contact with liquid acquisition screens conducted at the National Aeronautics and Space Administration (NASA) Glenn Research Center further suggest that an understanding of local thermodynamic state is essential to predict phase change. Much of the uncertainty in the liquid acquisition screen (LAD) experimental data was attributed to the evaporation/condensation at the screen surface 
[60 62]. In such a case, the liquid forms a meniscus and evaporation characteristics are much different from that of a planar surface. As the interfacial area decreases with respect to contact line length, the rate of evaporation is no longer proportional to the area. For a wetting system, the mass flux predicted from these planar models (such as Schrage's original model) reaches a singularity at the three phase contact point and an asymptotic treatment is necessary. In such a case, a multi-scale approach is needed to predict the mass flux based on both the macro- and micro-scale thermo/fluid physics taking place at the contact line region.

The contact line region is a continuously thinning film that terminates in an absorbed, non-evaporating layer. Figure 5.1 delineates regions of interest along a wetting evaporating meniscus based on the dominant component of normal stress that affects the thermo-fluid dynamics and their approximate length scales. The normal stress in the bulk is governed by capillary forces, or interface curvature. The adsorbed film region is most affected by intermolecular forces and is usually a nano-scale film. Both intermolecular forces and curvature affect the normal stress in the transition region. Further, resistance to thermal transport between the solid-liquid interface and the liquid-vapor interface increases with liquid film thickness. Hence, interfacial temperatures can vary significantly over the liquid-vapor interface.

Local variations in liquid-vapor interface curvature, interfacial temperature, and film thickness affect the rate of phase change. For non-polar wetting liquids, $60-90 \%$ of 


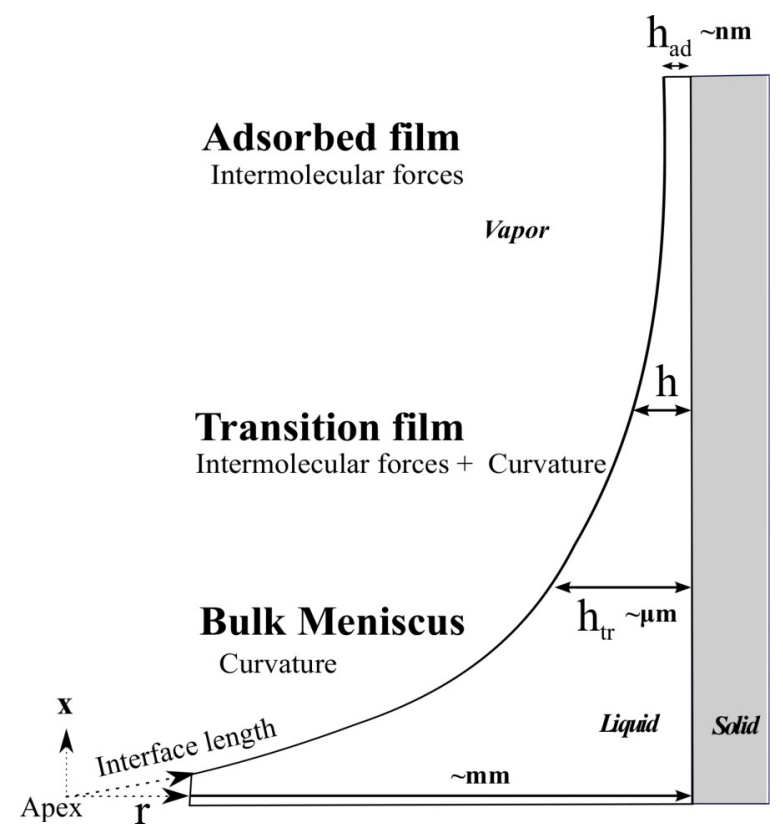

Figure 5.1: Regions of an evaporating wetting meniscus

the evaporation occurs in the transition film region close to the wall [43, 47, 49, 53, 55, 65, 93, 97, 106, 108, 109, 115, 121, 126, 127]. This is due to the interplay of thermal transport in the thin film and the dominating normal stress component at the interface. Anisotropy of the stresses in thin liquid films can be attributed to the disjoining pressure, which is a net pressure reduction in the nano-scale thin film due to intermolecular forces [47]. Curvature of the liquid-vapor interface gives rise to a capillary pressure jump. Hence, there is a variation in the local thermodynamic states in the contact line region that result in a non-uniform evaporation flux over the interface [106].

Several studies have been performed to determine the values of the accommodation coefficient, but the data reported is highly inconsistent [11, 46, 89, 95]. Even for a 
common fluid such as water, the reported values of coefficients vary by almost 3 orders of magnitude depending on the researcher or experimental method used [89]. There have been many attempts to investigate and explain the discrepancy of measured values for these coefficients, but no consensus has been reached [33, 45, 46, 89, 105]. As initially expressed by Cammenga et al. [33] and reiterated by Marek and Straub [89], the coefficient values published in literature vary depending on the material of the container used to conduct the experiment. This observation suggests that the wetting characteristics can affect the rate of evaporation and the corresponding computation of $\alpha$. In most of the prior studies the coefficient was determined using equations 5.1 or $5.2 \mathrm{~d}$ which are applicable only for a flat interface. Further, in these studies, the flat interface equations were evaluated with bulk properties of the fluid and not the local interfacial properties [5 88, 10, 44, 46, 84, 87, 119, 129]. The accommodation coefficient has been observed to vary with the length scale of the droplet or container [12, 96]. The partial lack of repeatability suggests an unknown parameter dependence [28].

In order to account for the geometrical effects while still using the equation for a flat interface, several researchers have included a shape factor for the accommodation coefficient. Burrows [29] noticed that the accommodation coefficient varies with geometry of the container used in the experiments and proposed a fit for the accommodation coefficient that depends on the area of the condensing surface and an empirically determined shape factor [29 32]. Kapłon et al. [73] claimed that the fit 
developed by Burrows [30] was inaccurate and proposed a new empirical fit. Similarly, Bryson et al. [27, 28] proposed their own empirical fits for the shape factor and the accommodation coefficients.

Wayner et al. [123, 124] adapted the planar kinetic model for phase change (equation 5.2d) for a curved interface. They used the Gibbs-Duhem equations for the bulk liquid and vapor phases coupled with surface tension to develop a fugacity 2 expression for the local interfacial thermodynamics. The expression was then integrated over a region where small changes in fugacity can be assumed to be equal to the corresponding change in vapor pressure. If the vapor density is neglected in comparison to the liquid density and thermal equilibrium is assumed over the interface, evaporation flux along a curved interface could be expressed by equation 5.3 [123, 124].

$$
\dot{m}^{\prime \prime}=\frac{2 \alpha}{2-\alpha}\left(\frac{M}{2 \pi R T_{\mathrm{i}}}\right)^{1 / 2}\left[\frac{p_{\mathrm{v}} M h_{\mathrm{fg}}}{R T_{\mathrm{v}} T_{\mathrm{i}}}\left(T_{\mathrm{i}}-T_{\mathrm{v}}\right)-\frac{v_{\mathrm{l}} p_{\mathrm{v}}}{R T_{\mathrm{i}}}(\Pi+\sigma \kappa)\right]
$$

where $\Pi$ is the disjoining pressure, $\sigma$ is surface tension, $h_{f g}$ is the enthalpy of vaporization, $R$ is the gas constant and $\kappa$ is the surface curvature, $p_{v}$ is vapor pressure, $M$ is the molar mass and $v_{l}$ is the molar volume of the liquid.

\footnotetext{
${ }^{2}$ Fugacity is a corrected vapor pressure that accounts for the "non-ideal" nature of a real gas.
} 


\subsection{Opportunity with Cryogenic Propellants}

The difference in the neutron attenuation coefficients between metals and cryogenic propellants allow for neutron imaging to be an ideal tool to visualize evaporation and condensation of hydrogenated propellants inside opaque metallic containers. In the experimental setup, temperature sensors could be mounted only on the outer wall of the test cell. Hence, a thermal model was used to characterize the heat transfer paths and determine inner wall solid-fluid interfacial temperature distributions from discrete outer wall temperature measurements. The experimental data from neutron imaging (Chapter 2 and 3) and corresponding solid-fluid temperature distributions from the thermal model (Chapter 4) allow for an opportunity to evaluate kinetic theory expressions and determine the value of $\alpha$. To the best of the author's knowledge, with the exception of this work, no measurements of $\alpha$ for cryogenic propellants are reported till date.

Due to the multi-scale nature of phase change, it is often difficult to develop a single modelling framework that works well at all length scales. To that effect, a multi-scale method to determine $\alpha$ is developed from a combination of a macro-scale model and a transition film model. The macro-scale model covers the bulk of the liquid-vapor interface but cannot resolve the thin film transition region close to the wall. This region is modeled using a thin film evaporation model. The rest of this chapter is 
dedicated to the multi-scale approach to determine $\alpha$ for liquid Hydrogen from a novel combination of experimental data and computational modeling.

\subsection{Neutron imaging experiments}

Phase change experiments with cryogenic propellants were conducted in the BT2 neutron imaging facility at the NIST Center for Neutron Research (NCNR) in Gaithersburg, MD. The huge variation in neutron attenuation between metals and cryogenic propellants allow for visualization of the liquid/vapor mixture inside opaque metallic containers [19, 23].

Cylindrical test cells of different sizes $(10 \mathrm{~mm}$ and $30 \mathrm{~mm}$ diameter $)$ and materials (Al 6061 and SS 316) were used in the experiments to test changes in both surface chemistry and curvature. By controlling both temperature and pressure, a range of phase change rates were observed at various thermodynamic conditions. Table 5.1 summarizes results from various test cells, the vapor pressure, temperature setting in the cryostat and corresponding phase change rates. In all these tests, the vapor pressure was kept constant during each test run and temperature is varied from $T_{\text {sat }}$ to induce condensation and/or evaporation. Figure 5.2 shows time lapse images captured during Run 1 using the $10 \mathrm{~mm}$ Al cell (corresponding to liquid volume shown in figure 3.12). Images 1-4 of figure 5.2 show condensation of liquid hydrogen 
Table 5.1

Summary of Cryo-Neutron tests conducted with $\mathrm{LH}_{2}$.

\begin{tabular}{|c|c|c|c|c|c|c|c|c|c|}
\hline Test Cell & $\begin{array}{l}\text { Test } \\
\text { Run }\end{array}$ & $\begin{array}{l}\text { Pressure } \\
(\mathrm{kPa})\end{array}$ & $\begin{array}{l}\mathrm{T}_{\text {sat }} \\
(\mathrm{K})\end{array}$ & $\begin{array}{l}\text { Cond. } \\
\text { Temp. } \\
(\mathrm{K})\end{array}$ & $\begin{array}{l}\text { Cond. } \\
\text { Rate } \\
(\mu \mathrm{g} / \mathrm{s})\end{array}$ & $\begin{array}{l}\text { Evap. } \\
\text { Temp. } \\
(\mathrm{K})\end{array}$ & $\begin{array}{l}\text { Evap. } \\
\text { Rate } \\
(\mu \mathrm{g} / \mathrm{s})\end{array}$ & $\begin{array}{l}\text { Surface } \\
\text { Area } \\
\left(\mathrm{mm}^{2}\right)\end{array}$ & $\begin{array}{l}\text { Contact } \\
\text { Line } \\
\text { Length } \\
(\mathrm{mm})\end{array}$ \\
\hline \multirow{5}{*}{$10 \mathrm{~mm} \mathrm{SS}$} & Run 1 & 120.9 & 20.98 & 19.9 & 70.62 & 22 & 16.05 & \multirow{5}{*}{152.42} & \multirow{5}{*}{31.4} \\
\hline & Run 2 & 88.32 & 19.91 & 18.8 & 56.13 & 21 & 17.16 & & \\
\hline & Run 3 & 201.96 & 22.95 & 21.9 & 59.21 & 23.5 & 21.14 & & \\
\hline & Run 4 & 204.03 & 22.99 & 22.5 & $\mathrm{~N} / \mathrm{A}$ & 24 & $\mathrm{~N} / \mathrm{A}$ & & \\
\hline & Run 5 & 204.03 & 22.99 & 22.5 & 34.25 & 28 & 76.39 & & \\
\hline $30 \mathrm{~mm} \mathrm{AL}$ & Run 1 & 121.94 & 21.01 & 20 & 81.14 & 24 & 102.8 & 1194.52 & 94.2 \\
\hline \multirow{4}{*}{$10 \mathrm{~mm} \mathrm{AL}$} & Run 1 & 121.3 & 20.99 & 19 & 116.6 & 23 & 55.2 & \multirow{4}{*}{152.42} & \multirow{4}{*}{31.4} \\
\hline & Run 2 & 87.9 & 19.89 & 17 & 149.8 & 22 & 55.5 & & \\
\hline & Run 3 & 204.38 & 23.01 & 20 & 214.4 & 26 & 92.9 & & \\
\hline & Run 4 & 200.05 & 22.91 & 20.5 & 167.2 & 26 & 77.12 & & \\
\hline
\end{tabular}

(shown in black) and images 4-8 show subsequent evaporation. Images from other tests look very similar. There is no observable hysteresis in the shape of the liquid vapor interface, within the spacio-temporal limits of the imaging system. Liquid hydrogen perfectly wets both $\mathrm{Al} 6061$ and SS 316 cells. Additional detail on the experiment setup, neutron image analysis, bulk evaporation rate $\left(\dot{m}_{\text {exp }}\right)$ and cryostat operation is detailed in the authors' previous publications [18, 19, 21 24, 77] and in chapters 2 and 3 .

The optical density method described in chapter 3 was used to estimate the equilibrium film thickness of the liquid meniscus. The film thickness variation with a set of condensation/evaporation images is shown in figure 5.3. The error in the film thickness measurement is dominated by neutron counting statistics and the standard deviation $(\sigma)$ is approximately $3.7 \mu \mathrm{m}$ in the case of liquid hydrogen. At the onset 

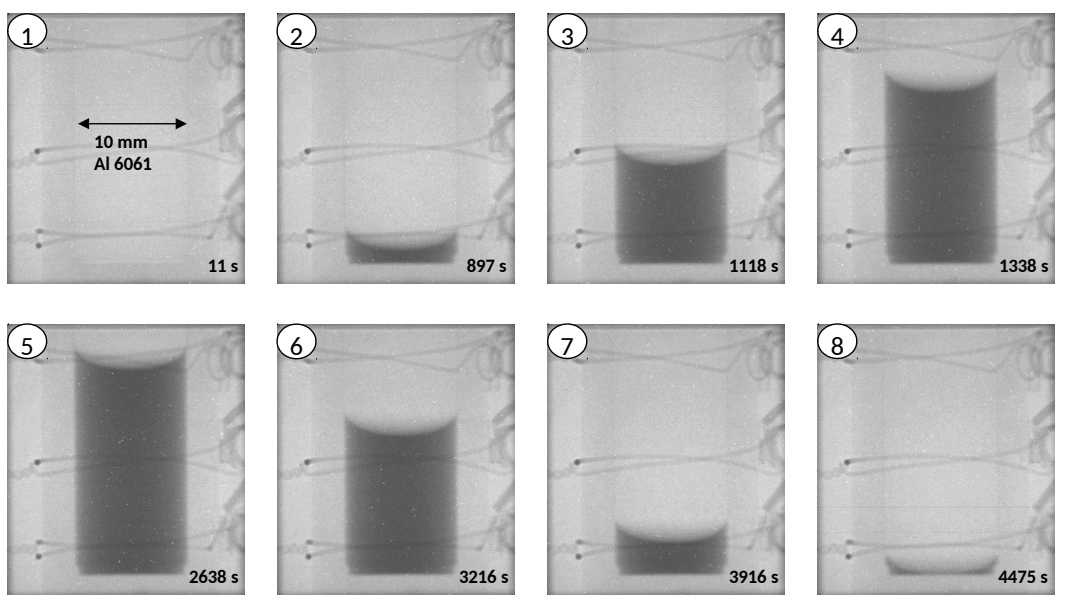

Figure 5.2: Time lapse images captured during Run 1 using the $10 \mathrm{~mm} \mathrm{Al}$ cell

of condensation, the film thickness begins to increase. In images 124-145, the bulk liquid at the bottom is in contact with the top corner meniscus, resulting in a thick continuous film (figure 5.3). During the condensation portion of the test run, film thickness greater than $10 \mu \mathrm{m}$ suggests that vapor could condense on the liquid film in addition to the bulk meniscus resulting in film drainage due to gravity. Accurate modeling of condensation in the experiments and subsequent determination of the accommodation coefficients would require a precise value of film drainage rates due to its inherent dependence on the interfacial mass flux. These drainage rates could not be determined using experimental data. However, during evaporation, the film thickness drops almost instantaneously to a value lower than $2 \sigma(7.4 \mu \mathrm{m})$, shown by horizontal dashed lines in figure 5.3. During evaporation, the error in film thickness measurement is greater than the magnitude of the measured value suggesting the possibility of a nano-scale adsorbed thin film. Hence, only the evaporation section of the experiments were used in the rest of this chapter to determine the accommodation 


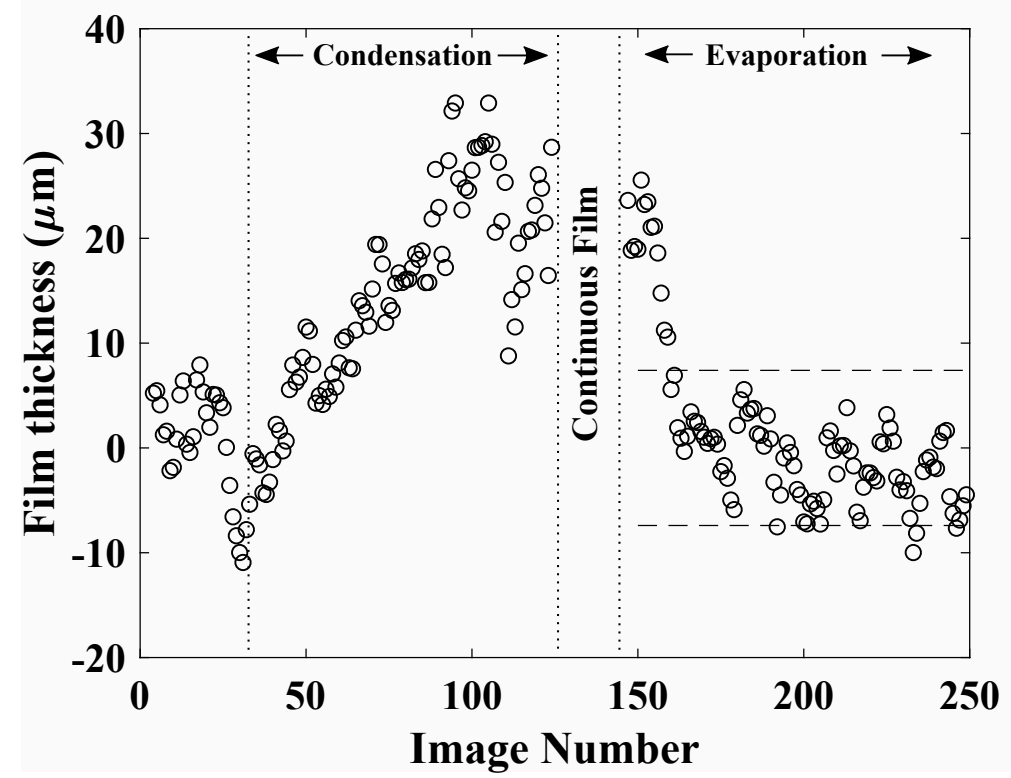

Figure 5.3: Film thickness measurements during Run 1 using the $10 \mathrm{~mm}$ SS 316 cell

coefficients.

\subsubsection{Thermal modeling of experiments}

Accurate modeling of evaporation requires the knowledge of the interfacial temperature. The temperature in turn affects the evaporation rate resulting in a complex, conjugate problem. In such a situation, the wall temperature could be specified and the heat transfer from the wall, through the liquid to the interface could be modeled. Most models assume a constant wall temperature for simplicity. It has previously been shown that due to the non-uniform evaporation flux in the contact line region, 
there exists a non-uniform wall temperature close to this region. Accurate modeling of evaporation requires the knowledge of the wall temperature distribution. Due to the nature of the experiments, temperature could be measured only along a few discrete locations on the outer wall of the test cells. In order to extract the inner wall solid-fluid interface distribution, the heat transport in the sample well must be characterized.

Dry thermal cycling tests were conducted during the experiments to investigate the modes and rates of heat transfer from the heater to the test cell. Heat is transfered from the heater to the test cell by a combination of (1) conduction along the baffle and the sample holder assembly, (2) conduction though the helium transfer gas and (3) convection through the helium gas (figure 5.4). The dry test data revealed that there is significant thermal resistance at all solid-solid interfaces in the sample well. A transient thermal transport model was built to determine the contact resistances that resulted in the best fit to the experimental results. Once the heat transfer parameters in the sample well were determined, the inner wall temperature distribution was extracted by using a heat sink at the interface with a value equal to $\dot{m}_{\text {exp }} h_{f g}$. Here, $\dot{m}_{\text {exp }}$ is the experimentally determined bulk evaporation rate and $h_{f g}$ is the enthalpy of vaporization. The shape of the interface and $\dot{m}_{e x p}$ is determined through image analysis. Details on the thermal model and the determination of the solid-fluid interface temperature can be found either in Chapter 4 of this dissertation or in Bellur et al. [20]. 


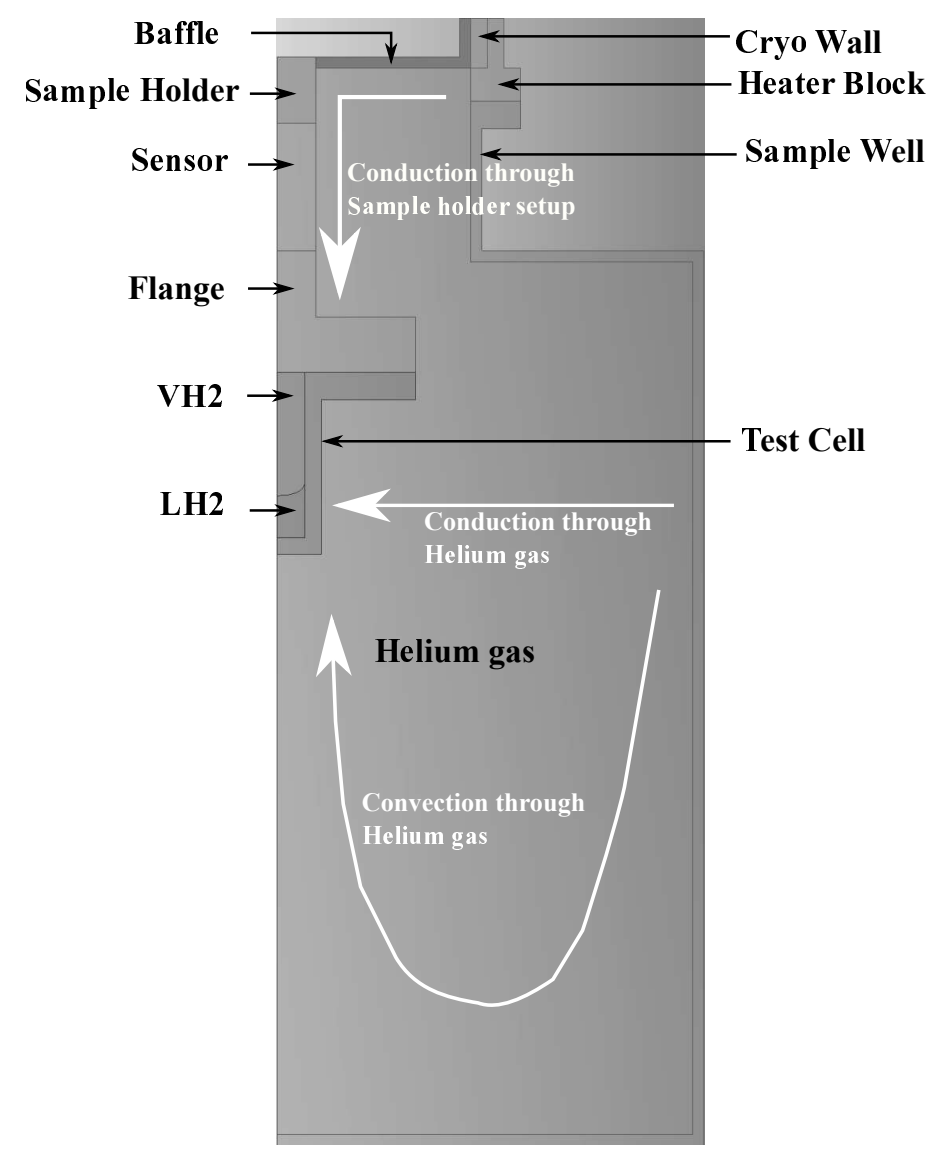

Figure 5.4: Heat transfer paths between the heater and the test cell.

\subsubsection{Macro-scale model of experiments}

The macro-model aims to capture the evaporative mass flux in the bulk of the interface (where capillary forces dominate) by modeling the transport processes in the liquid. The low evaporation rates measured in the experiments combined with geometry of the test cell suggest that the Rayleigh number was well within the critical Rayleigh number for natural convection and the Peclet number is estimated to be less than $10^{-2}$. The conductivity of liquid hydrogen is an order of magnitude greater than 
that of vapor. Evaporation results in a bulk movement of vapor molecules upward from the interface towards the outlet in the lid. The heat transport to the interface is dominated by conduction in the liquid and can be considered quasi-steady. Hence, a one sided, liquid only conduction model can effectively determine the liquid-vapor interface temperature from the solid-liquid interface temperature. Since the evaporation rate is low, the system could be considered quasi steady and a steady state model was implemented with a fixed liquid-vapor interface. A Young-Laplace fit to the liquid-vapor interface from the neutron images and the location of the meniscus apex is used to model the shape of the liquid-vapor interface. The inner wall temperature distribution on the side and bottom wall obtained from the thermal model (figure 4.10(b) is specified as a Dirichlet boundary condition. A Robin boundary condition is applied at the liquid-vapor interface (equation 5.4).

$$
Q_{l o s s}=h_{f g}\left(T_{i}\right) \dot{m}^{\prime \prime}\left(T_{i}\right)
$$

where, $Q_{\text {loss }}$ is the heat loss due to evaporation, and $\dot{m}^{\prime \prime}$ is the mass flux described by equation 5.3. The $2 \mathrm{D}$ axisymmetric, steady state heat conduction problem is set up in MATLAB and evaluated using the built-in finite element solver. A uniform mesh with $5 \mu \mathrm{m}$ triangular elements was used as a compromise between speed and accuracy. 


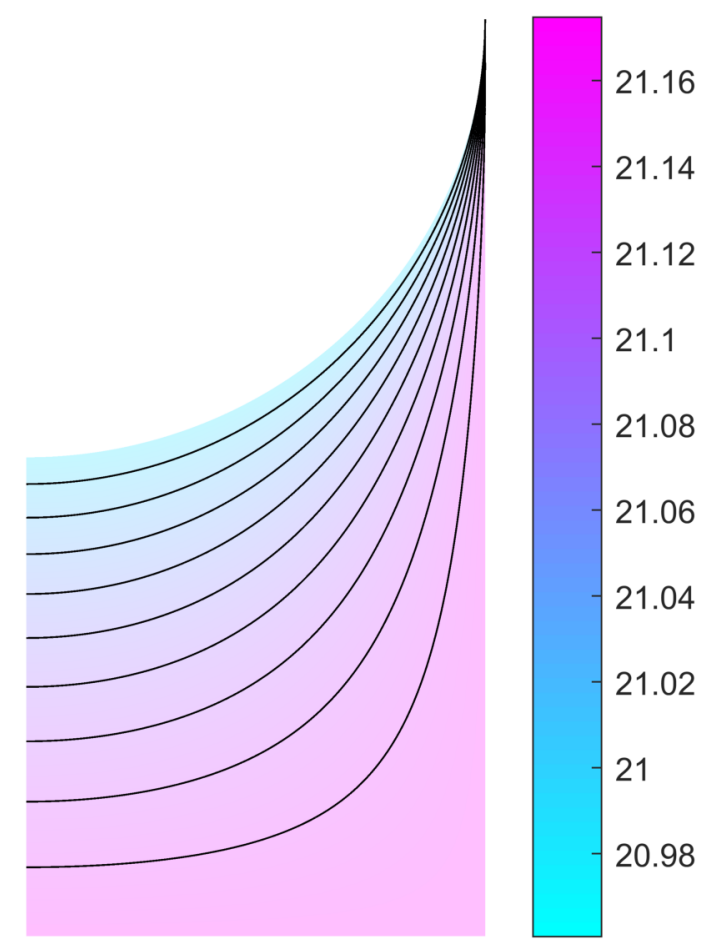

Figure 5.5: Contour of temperature results from the Macro-model for Run 2 using the $10 \mathrm{~mm} \mathrm{Al}$ cell. The result shown here corresponds to the final converged value of $\alpha=0.58$

A contour plot of the results and the boundary conditions are shown in figure 5.5 . Figure $5.6(\mathrm{a})$ shows the variation in the interfacial temperature and figure 5.6(b) shows the variation in mass flux along the meniscus arc length, starting at the apex of the meniscus and truncated at a film thickness of $10 \mu \mathrm{m}$. In the bulk meniscus, the interfacial temperatures remain fairly constant and close to $T_{\text {sat }}$ which was experimentally determined from the pressure measurement to be $20.99 \pm 0.03 \mathrm{~K}$. As one moves along the interface from the bulk to the thin film region several factors change simultaneously: 


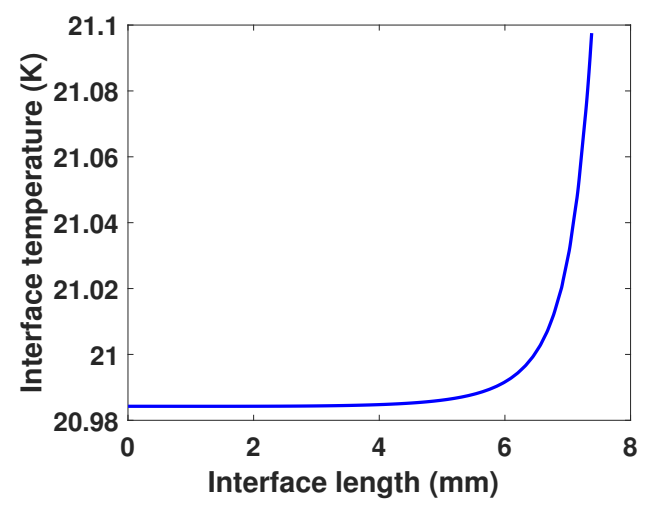

(a) Temperature

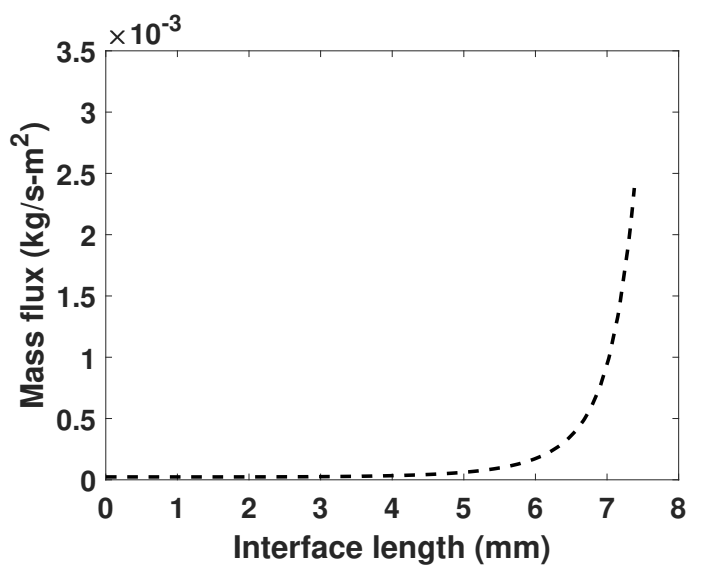

(b) Mass flux

Figure 5.6: Local interfacial temperature and mass flux along the liquidvapor interface corresponding to results in figure 5.5. Zero value on the $\mathrm{x}$ axis refers to the apex of the meniscus. Results for film thickness $<10 \mu \mathrm{m}$ are neglected.

(1) The resistance to heat transfer is reduced due to thinning of the liquid film. This results in an increase in interfacial temperature.

(2) An increased interfacial temperature results in an increase in local evaporation flux (equation 5.3).

(3) The enhanced evaporation results in an increased cooling of the interface due to latent heat of phase change.

(4) For Bond numbers less than 1, the curvature of the liquid vapor interface is inversely proportional to film thickness. As a result, the increased curvature causes a decrease in evaporation flux (equation 5.3).

All the above effects are intrinsically related. The change in evaporation flux due to curvature is usually negligible in comparison to the thermal contribution at high 
superheats and small Bond numbers. In this case, the superheat is low $(<0.1 \mathrm{~K})$ and the Bond number is approximately 9.8 and ignoring the curvature effect varies the local mass flux by up to $10 \%$.

The 2D macro-model albeit simple provides a reasonably good description of the mass flux along the interface. There are, however, three major drawbacks: (1) Hydrogen is a perfectly wetting fluid with a contact angle of zero. This suggests that the film spreads infinitely and that a thin non-evaporating absorbed film exists. However, the Young-Laplace fit with a zero degree contact angle generates a theoretical curve that approaches a film thickness of zero at a finite distance, which is not physical. If the fitting curve is terminated at an assumed value of adsorbed film thickness, an extremely fine mesh must be resolved. The modeling results are sensitive to the assumed adsorbed film thickness. (2) Without an adsorbed film, the problem is inherently mesh dependent. As the mesh is refined, the temperature peak close to the wall increases. The integral of the mass flux along the interface is dependent on the size of mesh in the thinnest region of the liquid. (3) The Young-Laplace fit is not accurate at sub-micron thicknesses. When the liquid-vapor interface is in close proximity to the solid-liquid interface, disjoining pressure alters the local pressure field thereby altering both the mechanical stress balance (interface shape) and the local evaporation flux. Hence, the macro-model is not suited for evaluation at close to the wall and a secondary model is necessary to investigate evaporation in the transition film region. 
The macro-model is thus used to evaluate the evaporation rate from the bulk of the liquid-vapor interface and is truncated at a film thickness of $10 \mu \mathrm{m}$. The local mass flux in figure 5.6(b) is integrated over the entire liquid-vapor surface area to obtain the contribution to the total evaporation rate from the macro-model $\left(\dot{m}_{m m}\right)$.

\subsection{Transition film model}

As discussed in section 5.1, most of the evaporation in a wetting meniscus occurs in the transition region close to the wall. While the macro-model accounts for evaporation in the bulk meniscus, the transition film model aims to bridge the gap from the beginning of the transition region $(10 \mu \mathrm{m}$ thick film) to the adsorbed thin film (nm thick film). The different regions of the meniscus are shown in figure 5.1 .

The mechanical pressure balance in the thin film can be modeled using the augmented Young-Laplace equation that accounts for both the curvature and the disjoining pres-

sure. Equation [5.5 developed by DasGupta et al. [42], describes the local pressure jump across the liquid-vapor interface.

$$
p_{v}-p_{l}=\sigma \kappa+\Pi
$$


Here $p_{v}$ is the pressure in the vapor phase and $p_{l}$ is the pressure in the liquid phase. The fluid properties and local pressure in the vapor could be assumed to be uniform and constant throughout the domain resulting in a change in liquid pressure that could be expressed explicitly in terms of $\kappa$ and $\Pi$ which are in turn dependent on local liquid film thickness.

The geometry of interest has two planes of curvature, one due to the meniscus and the other due to the radius of the container. The geometric curvature at any location on the liquid vapor interface is,

$$
\kappa=(r-h)^{-1}\left(1+h_{x}^{2}\right)^{-1 / 2}+h_{x x}\left(1+h_{x}^{2}\right)^{-3 / 2}
$$

where, $\kappa$ is the curvature, $h$ is the liquid film thickness, $h_{x}$ is the first derivative, $h_{x x}$ is the second derivative, $x$ is the vertical distance with origin at the liquid-solid interface.

The disjoining pressure is modeled using equation 5.7 considering only the intermolecular London-Van Der Waals forces [47].

$$
\Pi=\frac{A}{h^{3}}
$$


where $\Pi$ is the disjoining pressure, $A$ is the Hamaker constant. Typical values of $A$ constant for different fluids are between $10^{-19}$ to $10^{-22} \mathrm{~J}$.

Dzyaloshinskii et al. [50] developed a general theory of Van Der Waals forces using a quantum field approach that suggests that the value of $A$ is not truly a constant but is a complex function that depends on temperature, liquid film thickness, frequency etc. This requires a complete mapping of the complex dielectric permittivity and other optical properties for all frequencies of electromagnetic radiation though liquid hydrogen and is beyond the scope of the current project. Holm and Goplen [65] developed an alternative model for polar fluids such as water but this requires the knowledge of empirically determined constants. Wu and Wong [130] presented a slope dependent disjoining pressure model for non-zero contact angles. In this work, equation 5.7 is used with $A=5.11 \times 10^{-21}[70]$.

Substituting equations 5.6 and 5.7 into 5.5 and differentiating, a non-linear thin film evolution equation as described by equation 5.8 is obtained.

$$
\begin{gathered}
h_{x x x}-\frac{3 h_{x x}^{2} h_{x}}{1+h_{x}^{2}}-\frac{h_{x x} h_{x}}{\left(r_{i j}-h\right)^{2}}+\frac{h_{x}\left(1+h_{x}^{2}\right)}{\left(r_{i j}-h\right)^{2}}+ \\
\frac{\gamma}{\sigma}\left(\frac{1+h_{x}^{2}}{r_{i j}-h}+h_{x x}\right) \frac{d T}{d x}+\frac{1}{\sigma}\left(1+h_{x}^{2}\right)^{\frac{1}{2}}\left(\frac{d p_{l}}{d x}+\frac{d \Pi}{d x}\right)=0
\end{gathered}
$$

Liquid flow in the transition film (figure 5.1) is modeled using a lubrication approximation of the Navier-Stokes equation in cylindrical coordinates, 


$$
\frac{1}{r} \frac{\partial}{\partial r}\left(r \frac{\partial u}{\partial r}\right)=\frac{1}{\mu_{l}} \frac{d p_{l}}{d x}
$$

where $\mu_{l}$ is the viscosity of the liquid, $u$ is velocity, $r$ is the local radius and $d p_{l} / d x$ is the pressure gradient. The equation is solved by applying a no-slip boundary condition at the wall and a tangential stress boundary condition at the interface. A temperature dependent surface tension is used to account for Marangoni effects.

$$
\begin{aligned}
\text { at } r & =R, & u & =0 \\
\text { at } r & =R-h, & -\left.\mu \frac{\partial u}{\partial r}\right|_{r=R-h} & =\frac{d \sigma}{d x}
\end{aligned}
$$

Upon solving equation 5.9 using the given boundary conditions, an expression for velocity, $u(r)$, is obtained. The mass flow rate through a control volume $\left(\dot{m}_{c v}\right)$ in the transition film region is,

$$
\dot{m}_{c v}=\int_{R-h}^{R} \rho_{l}[u(r)] 2 \pi r d r
$$

The difference in the mass flow rate entering and exiting the control volume is set equal to the evaporative flux evaluated using the kinetic model (equation $5.2 \mathrm{~d}$ ). From this balance, the pressure gradient $d p_{l} / d x$ is obtained. 
An energy balance on same transition film control volume is,

$$
k_{l} \frac{\partial}{\partial r}\left(r \frac{\partial T}{\partial r}\right)=0
$$

A constant wall temperature boundary condition along with a heat flux boundary condition is used to solve the equation. The heat flux accounts for the conduction and the energy lost due to evaporation.

$$
\begin{aligned}
\text { at } r & =R, & T & =T_{\text {wall }} \\
\text { at } r & =R-h, & k_{l} \frac{d T}{d r} & =\dot{m}^{\prime \prime} h_{f g}
\end{aligned}
$$

Integrating equation 5.11 from wall, $R$, to the interface, $R-h(x)$, the interfacial temperature distribution is obtained.

$$
T_{i}(x)=-\frac{h_{f g}}{k_{l}}(R-h(x)) \ln \left(\frac{R}{R-h}\right) \dot{m}^{\prime \prime}+T_{\text {wall }}(x)
$$

where, $\dot{m}^{\prime \prime}$ is evaluated using equation 5.3 and $T_{\text {wall }}$ is the solid-fluid interface temperature distribution obtained from the thermal model. 
Equations [5.3, 5.5 - 5.8, 5.10 and 5.12 must be evaluated numerically between the adsorbed film region $\left(h_{a d}\right)$ to the start of the bulk meniscus $\left(h_{t r}\right)$. The third order evolution equation in the thin film requires three boundary conditions. The mass balance requires an additional boundary condition that may be specified either at $h_{t r}$ or $h_{a d}$. In order to obtain the thermal boundary conditions $\left(T_{\text {wall }}(x)\right)$, the thermal model must be used. Evaluating the thermal model requires the knowledge of $\dot{m}^{\prime \prime}$ over the entire interface. However, it has previously been shown that $60-90 \%$ of the evaporation occurs in the thin film region close to the wall [43, 47, 53, 65, 106, 108, 127]. Hence, the thermal model is initially evaluated with the assumption that all of the evaporation occurs in a $1 \mathrm{~mm}$ region close to the wall as previously described in chapter 4. This assumption is relaxed in subsequent iterations but the change in the values of $T_{\text {wall }}$ the resulting value of $\alpha$ were both $<1 \%$.

The computational domain size, i. e. length and thickness gradients of the transition film region, is not known a priori. One approach is to set the value of $h_{a d}$ and the corresponding film thickness derivatives so that a shooting method may be used to evaluate the film profile from the adsorbed film. The profile is matched to a specified bulk curvature at an arbitrary length from $h_{a d}$ [53, 115, 127].

This method involves iterating on three parameters $\left(h_{a d}, h^{\prime}, h^{\prime \prime}\right)$ in order to find a feasible solution. In order to match the bulk curvature, researchers in the past have "tuned" a combination of $h_{a d}$ and its derivatives and/or slip length [25, 53, 56, 71, 
98, 107, 127]. Even if a matched curvature solution may be obtained, the resulting initial conditions have a great potential to be non-physical and/or non-unique.

Recently, a method to integrate the evolution equation starting from the curvature region and ending at the adsorbed film region has been demonstrated by Akkuş and Dursunkaya [2]. This approach begins with an initial value of mass flow into the transition film region. The set of equations describing mass, energy and momentum in the transition film region (equations 5.3, 5.5 - 5.8, 5.10 and 5.12) are evaluated until $h_{x}=0$. The film thickness at which $h_{x}=0$ is presumed to be $h_{a d}$. Because the evaporative flux is zero in the adsorbed film region, the initial mass flow of liquid must completely evaporate as $h_{x} \rightarrow 0$ along the transition film. This constraint serves as an additional boundary condition. If the film thickness and its derivatives at the thick film $\left(h_{t r}\right)$ are known (experimentally measured), the correct mass flow into the transition region is determined iteratively. This alleviates the need for guessing multiple boundary conditions at the adsorbed film. The local mass flux obtained as a result of the transition film model is integrated over the interfacial area of the transition film region to obtain $\dot{m}_{t f m}$.

The methodology proposed by Akkuş and Dursunkaya [2] was used to evaluate the transition film model. Using $h_{t r}=10 \mu \mathrm{m}$ and the values of the derivatives at that point $\left(h_{x}\right.$ and $\left.h_{x x}\right)$ determined from Young-Laplace fits to the neutron images as initial conditions, the transition film model (Equations 5.3, 5.5, 5.6, 5.8, 5.10 and 
5.12) are evaluated in the direction of reducing film thickness until the local value of $h_{x}$ reaches zero. Figure 5.7 shows the film profile obtained as a result of the transition film model for a $10 \mathrm{~mm} \mathrm{Al}$ cell containing hydrogen evaporating at $121.3 \mathrm{kPa}$ (Run 1) with $\alpha=0.58$. The value of $\alpha$ chosen corresponds to the final converged value. In figure 5.7, the origin corresponds to the solid-liquid interface at a film thickness of $10 \mu \mathrm{m}$. As the film thickness reduces, the modeling results deviate from the YoungLaplace fit, which is valid only in the bulk meniscus region. The inset of figure 5.7 shows the model ending in a non-evaporating adsorbed film while the Young-Laplace fit ends in a zero film thickness. Once $h_{x}=0$ is obtained, solution is terminated. An adsorbed film thickness of approximately $70 \mathrm{~nm}$ is observed.

The adsorbed film thickness obtained is sensitive to the model of disjoining pressure used. The adsorbed film thickness also varies with the experimental test conditions and the geometry of the test cell but lies between $50 \mathrm{~nm}$ and $80 \mathrm{~nm}$ for the set of conditions simulated. Further work is necessary to investigate the effect of different disjoining pressure models and the experimental conditions on the value of $h_{a d}$.

\subsection{Computing the Accommodation Coefficient}

The value of evaporative mass obtained from transition film model and the evaporative mass obtained from the macro-model are both dependent on the accommodation 


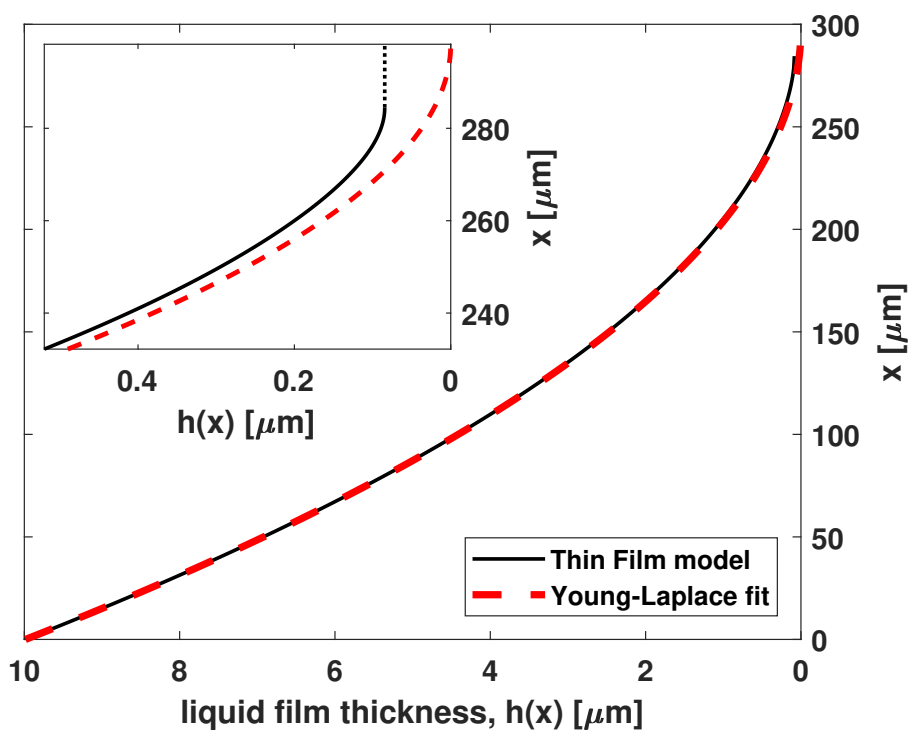

Figure 5.7: Solution of the transition film model for a $10 \mathrm{~mm}$ Al cell containing hydrogen evaporating at $121.3 \mathrm{kPa}, \alpha=0.58$.

coefficient. The algorithm shown in figure 5.8 is used to determine the value of $\alpha$ in that results in a total evaporative mass (sum of masses from macro-model and the transition film model) that matches the experimentally determined bulk value $\left(\dot{m}_{e x p}\right)$. The value of $\alpha$ is initially assumed to be 0.5 and the macro-model results are truncated at a film thickness of $10 \mu \mathrm{m}$. The transition film model is then evaluated starting $10 \mu \mathrm{m}$ and terminated at $h_{a d}$ using the methodology described in the previous section. The value of $\alpha$ is varied until the condition $\dot{m}_{t f m}+\dot{m}_{m m}=\dot{m}_{e x p}$ is satisfied.

Figure 5.9 shows the mass flux distributions from the coupled multi-scale model and liquid film thickness along the interface. Interface length equal to zero corresponds to the apex of the meniscus. Moving along the interface away from the apex, the film thickness reduces as shown in figure 5.9, The macro-model results are truncated at $10 \mu \mathrm{m}$ and this also serves as the starting point for the transition film model. The 


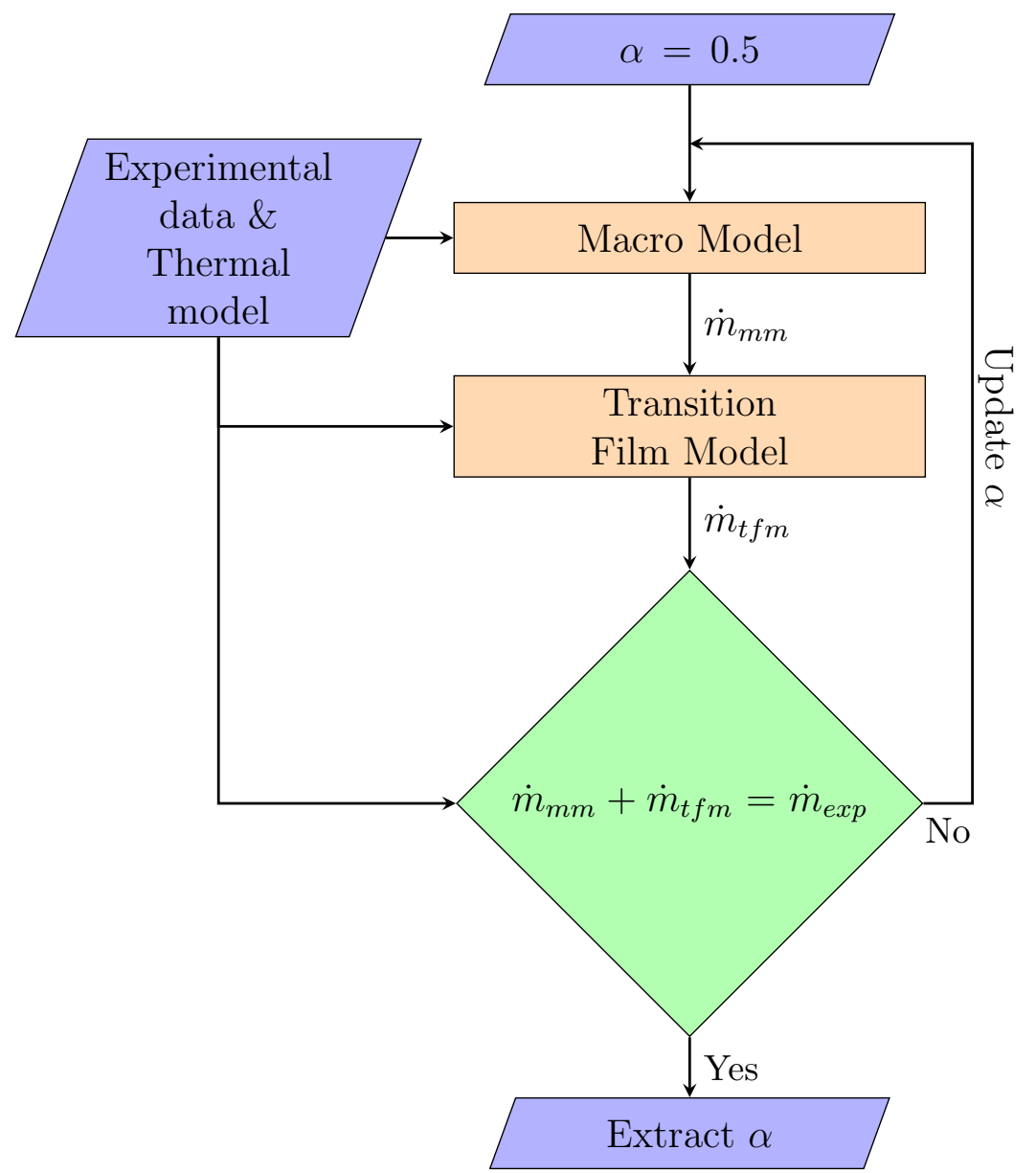

Figure 5.8: Algorithm flowchart

mass flux profile is continuous and smooth at the matching point $\left(h_{t r}\right)$. The coupled solution exhibits a peak in mass flux in the transition film region, as expected. At film thicknesses close to $h_{a d}$ the mass flux quickly drops to zero. Figure 5.10 shows the variation in the value of $\dot{m}_{t f m}+\dot{m}_{m m}$ with $\alpha$. There exists a unique value of $\alpha$ for a given set of experiment conditions.

A sensitivity analysis was performed to investigate the effect of initial value of $\alpha=0.5$ on final value of $\alpha$. It was found that the final value is insensitive to the initial value 


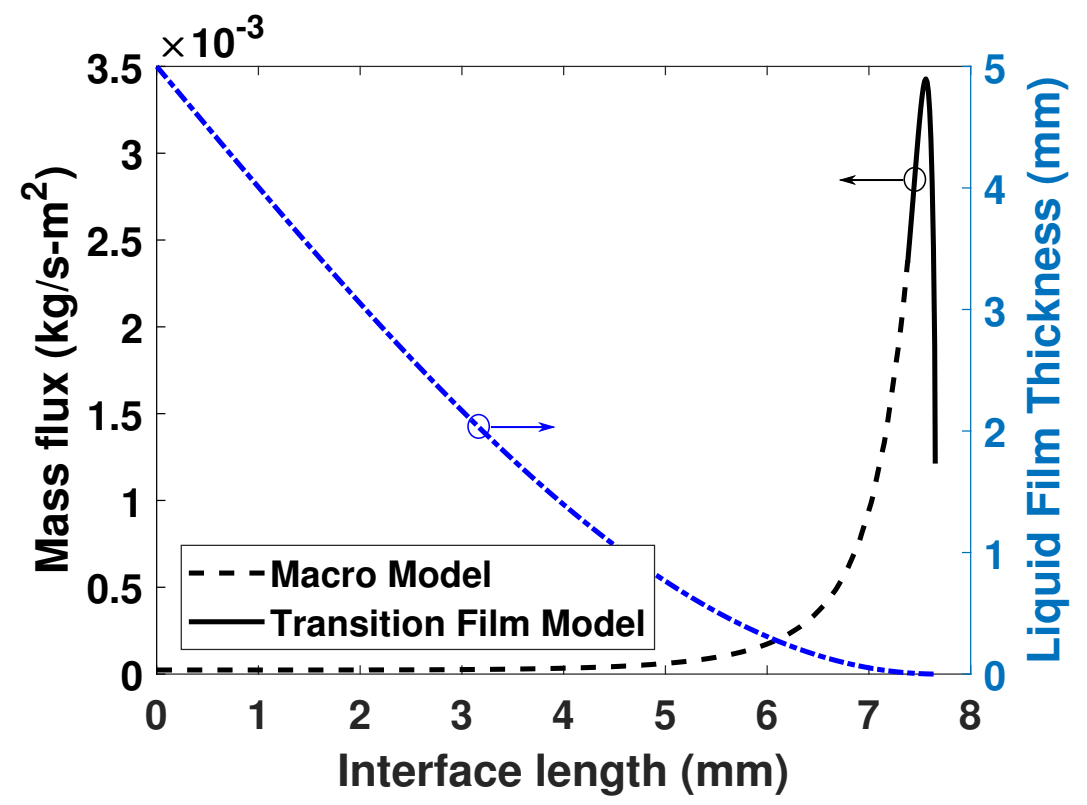

Figure 5.9: Combined local mass flux values from the coupled multi-scale model for a $10 \mathrm{~mm} \mathrm{Al}$ cell containing hydrogen evaporating at $121.3 \mathrm{kPa}$, $\alpha=0.58$

of $\alpha$ if the initial is lower than the final value. If the initial value is greater than $30 \%$

of the final value, then depending on the root finding method used, the algorithm may not converge.

\subsection{Accommodation Coefficients of $\mathrm{LH}_{2}$}

The value of the accommodation coefficient was determined for cryogenic phase change tests of liquid hydrogen. Test runs listed in table 5.1 were analyzed and corresponding accommodation coefficients were determined (figure 5.11 and table 5.2). Both the Hertz-Knudsen and Schrage forms of equation 5.3 were tested. The only 


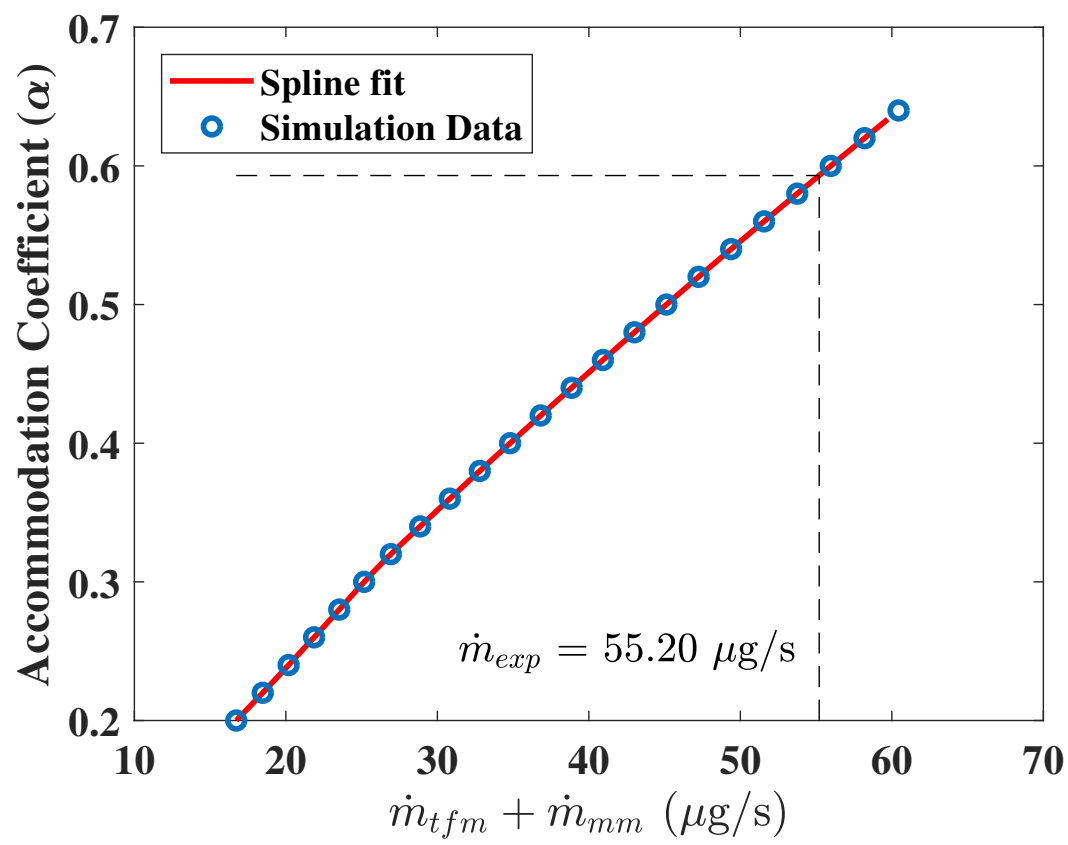

Figure 5.10: $\alpha$ vs $\dot{m}_{t f m}+\dot{m}_{m m}$ for a $10 \mathrm{~mm} \mathrm{Al} \mathrm{cell} \mathrm{with} \mathrm{hydrogen} \mathrm{evapo-}$ rating at $121.3 \mathrm{kPa}$ (Run 1$)$.

difference between Hertz-Knudsen and Schrage expressions considered here is that the kinetic pre-factor $\frac{2 \alpha}{(2-\alpha)}$ in the Schrage from of equation 5.3 is replaced by $\alpha$ to develop the Hertz-Knudsen form of equation 5.3 .

The uncertainty associated with $\alpha$ is due to a combination of uncertainty in temperature and pressure measurements, imaging uncertainty in determining bulk evaporation rates, and numerical error in the model. The dominant factor in the uncertainty of $\alpha$ is due to the temperature sensors used in the experiments, which was $\pm 0.25 \mathrm{~K}$. This uncertainty is at least two orders of magnitude greater than all other sources.

The general trend in figure 5.11 indicates that $\alpha$ decreases with saturation vapor pressure. This trend is in agreement with data published by previous researchers for 


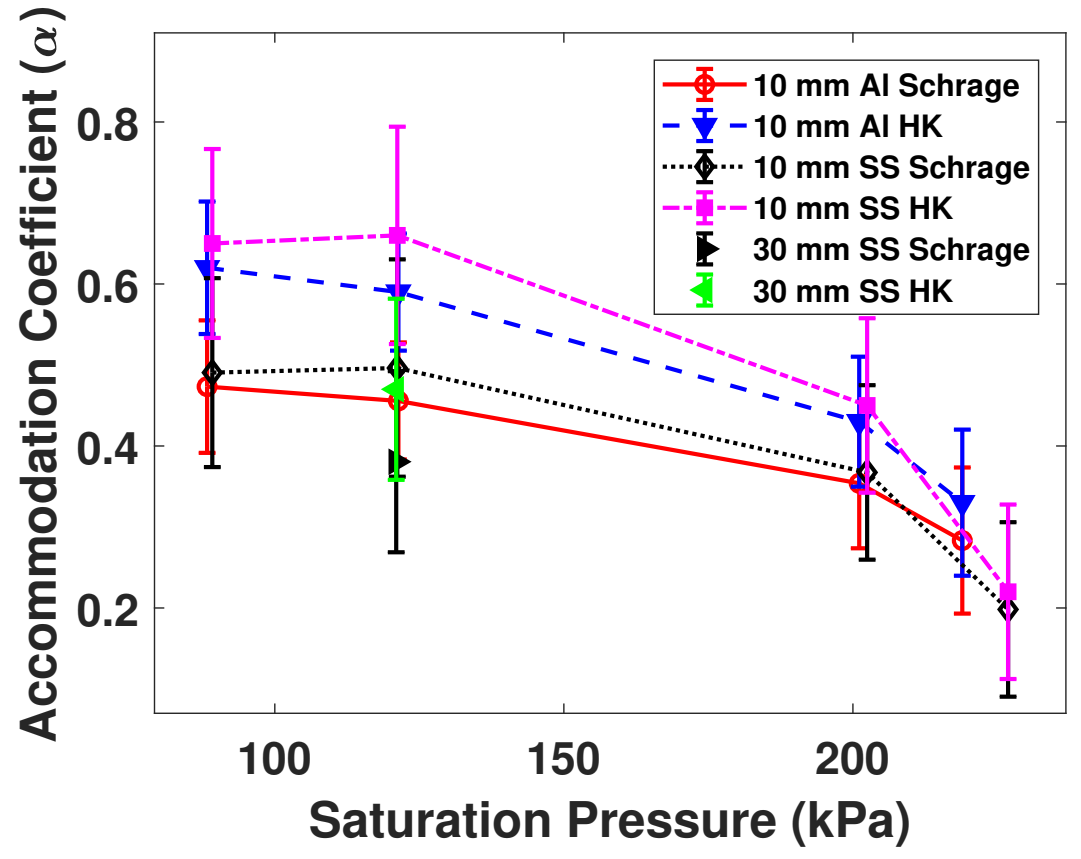

Figure 5.11: $\alpha$ vs Vapor Pressure for $\mathrm{LH}_{2}$

evaporation coefficients of water [89].

Variation in $\alpha$ due to size of the test cell is within the measurement uncertainty, as seen in table 5.2. This suggests that the new methodology accurately captures the effect of bulk curvature and the contact line length. Comparing the results for the $10 \mathrm{~mm} \mathrm{SS}$ to the $10 \mathrm{~mm} \mathrm{Al}$ cell, it is seen that with the given uncertainity, there no evident material dependence in the value of $\alpha$. Since hydrogen is perfectly wetting to both $\mathrm{Al} 6061$ and SS 316 and there is no change in either the disjoining pressure or the bulk curvature if the cell size is kept constant.

There is good agreement with the Hertz-Knudsen and Schrage forms of equation 5.3 at lower values of $\alpha$, which indicates that the effect of drift velocity increases with $\alpha$ 
Table 5.2

The determined values of $\alpha$ for all test runs with hydrogen

\begin{tabular}{ccccc}
\hline Test Cell & Pressure $(\mathrm{kPa})$ & $\dot{m}_{\exp }(\mathrm{g} / \mathrm{s})$ & $\alpha-\mathrm{HK}$ & $\alpha$ - Schrage \\
\hline \multirow{3}{*}{ 10mm SS } & 89.19 & 17.16 & $0.53-0.76$ & $0.37-0.61$ \\
& 121.19 & 16.05 & $0.53-0.79$ & $0.36-0.63$ \\
& 202.46 & 21.14 & $0.34-0.56$ & $0.26-0.47$ \\
\hline \multirow{3}{*}{ 30mm AL } & 226.84 & 76.39 & $0.11-0.33$ & $0.09-0.31$ \\
\hline \multirow{3}{*}{ 10mm AL } & 121.94 & 102.8 & $0.36-0.58$ & $0.27-0.49$ \\
& 128.29 & 55.5 & $0.54-0.70$ & $0.39-0.56$ \\
& 201.09 & 55.2 & $0.52-0.66$ & $0.38-0.53$ \\
& 218.92 & 77.12 & $0.35-0.51$ & $0.27-0.43$ \\
\hline
\end{tabular}

and/or decreases with vapor pressure. The possible increase in the drift velocity raises concerns regarding the validity of the commonly used approximation $\mathcal{T}(a) \approx 1+a \sqrt{\pi}$ at sub-atmospheric pressures. The Schrage equation is more accurate compared to the Hertz-Knudsen equation. Hence, for the same value of $\alpha$, the Hertz-Knudsen equation may under predict the mass flux.

\subsection{Summary and Conclusion}

The values of the accommodation coefficient for liquid hydrogen are necessary to predict boil-off in both space (large fuel depots in orbit, fuel management for long term missions) and terrestrial (ground transport of and storage for the hydrogen economy) applications. In this work, a novel technique to determine the accommodation 
coefficient of hydrogen is detailed. The technique is a combination of experimental and computational efforts. The experimental effort utilizes neutron imaging as a visualization tool to measure the evaporation rates under various conditions. The computational effort is a mix of thermal transport modeling in the test setup combined with a multi-scale model of phase change at different length scales.

Accommodation coefficient(s) were determined for hydrogen at various thermodynamic conditions (vapor pressures, evaporation rates) and test cells (varied size and material). The conclusions are summarized as follows:

1. If the wetting and curvature were captured accurately, any variation in $\alpha$ due to geometry or container material is within the measurement error.

2. The uncertainty in $\alpha$ is dominated by the uncertainty in the temperature measurement $( \pm 0.25 \mathrm{~K})$.

3. The evaporation rate itself has no noticeable effect on the value of $\alpha$.

4. $\alpha$ decreases with increasing vapor pressure. 


\section{Chapter 6}

\section{Summary}

Although kinetic theory has been used to describe liquid-vapor phase change for at least a century there is much discrepancy in the values of the evaporation and condensation coefficients (also known as accommodation coefficients). Even for a common fluid such as water, the reported values of coefficients vary by almost 3 orders of magnitude depending on the researcher or experimental method used [89]. There have been many attempts to investigate and explain the discrepancy of measured values for these coefficients but no consensus has been reached [33, 45, 46, 89, 105]. There have been no measurements of accommodation coefficients for cryogenic propellants till date. The values of the accommodation coefficient for liquid hydrogen are necessary to predict boil-off in both space (large fuel depots in orbit, fuel management for long term missions) and terrestrial (ground transport of and storage for the hydrogen 
economy) applications. Further, there have been no experiments of "controlled" phase change with cryogenic propellants. To that effect, a new methodology to (1) image cryogenic propellants, (2) develop a new protocol for "controlled" phase change tests with cryogenic propellants, (3) investigate wettability of cryogenic liquids and (4) determine accommodation coefficients for a wide variety of test conditions is presented in this work.

A new method to visualize cryogenic liquids inside opaque metallic containers using neutron imaging is described in Chapters 2, 3 and 4. Chapter 2 contains details on the experimental setup and Chapter 3 contains details on the post-processing and analysis of the acquired images. Cryogenic propellants have been predicted to be perfectly wetting but direct optical measurements of the contact angles of hydrogen or methane were not found in literature. Contact angle measurements of liquid hydrogen and methane are presented in Section 3 of Chapter 2, Section 4 of Chapter 3 and chapter 7. The fidelity of the data analysis and image processing was gradually increased but the error in the contact angle measurement from the best conventional image processing techniques were $\pm 2^{\circ}$. In 2017, I developed a method to probe thin liquid films with length scales smaller than the spatial resolution using a neutron attenuation analysis (demonstrated in Chapter 7). This method finally proves that liquid hydrogen and methane are perfectly wetting with a contact angle of $0^{\circ}$. I'm currently in the process of writing a new manuscript focusing on this method, the results and its implications. 
There was no published work on low temperature heat transport through different parts of a cryostat. Most of the prior work with a cryostat involved a steady state analysis. In order to obtain high resolution temperature boundary conditions, I explored the transient heat transfer characteristics in detail and developed a corresponding methodology to model the thermal transport (presented in Chapter 4). The methodology was then expanded to determine non-uniform temperature distribution from discrete temperature measurements. Lastly, I developed a multi-scale model to account for phase change (presented in Chapter 5). The uniquely coupled multi-scale model combined with experimental data allows for the determination of the accommodation coefficient. Dependence of the accommodation coefficient on saturation conditions, curvature and surface chemistry is investigated and explored. It is my opinion that the 3 orders of magnitude spread of the prior measurements is predominantly due to the failure to account for the non-uniform evaporation flux and temperature distributions that arise from curvature, disjoining pressure and other multi-scale effects.

To my knowledge, this work includes the first ever reported: (1) images, (2) optically accessible phase change tests, and (3) values of the accommodation coefficients of cryogenic propellants. The author hopes that this opens the door for several advances in the field. Many avenues of future work are detailed below. 


\subsection{Accommodation coefficients of methane}

Neutron imaging phase change experiments were conducted with liquid methane in

July and September 2015. However, the subsequent analysis of the methane experiments has been delayed in favor of completion of the hydrogen analysis. The next immediate task is to repeat the analysis described for methane for comparison and contrast.

\subsection{Effect of non-condensible gases}

One of the most popular active boil-off control strategies is to inject liquid helium into liquid hydrogen tanks in order to cool, relieve pressure buildup and condense hydrogen vapor. This results in a mixture of hydrogen and non-condensible helium in the vapor ullage. Additional experiments with a non-condensible gas could provide further insight on the effect of a non-condensible gas on the thermophysics of phase change. 


\subsection{High resolution temperature sensors}

One of the conclusions of this work is that the error in the accommodation coefficient is dominated by the error in the temperature sensors. Repetition of similar experiments with additional high resolution temperature sensors would greatly reduce the uncertainty in the coefficients. Any future measurements of the accommodation coefficients or modeling of phase change will require precise temperature measurements especially in the vicinity of the contact line.

\subsection{Effect of disjoining pressure}

In this work the simplest polynomial expression for the disjoining pressure in terms of a Hamaker constant has been used (equation 5.6). While this is a good approximation, it has previously been shown that the Hamaker constant is in fact a function that depends on temperature and film thickness. A complete description of the disjoining

pressure based on quantum field theory is given by Dzyaloshinskii et al. [50]. Future studies could include such non-linear effects into the current modeling framework. The extent to which the disjoining pressure varies the accommodation coefficient is currently unknown. 


\subsection{Testing of assumptions}

Due to the explosive nature of the propellants, experiments could be done only in an "open" configuration. To ensure closure of the set of equations, the evaporation coefficient was assumed to be equal to the condensation coefficient as has been done by many researchers in the past [11, 43, 44, 66, 89, 95, 106, 123 125]. However, the author recognizes that this assumption may not always be valid. Kryukov and Levashov [81] concluded that assuming $\alpha_{e}=\alpha_{c}$ "increases the error of evaporation-condensation strongly". Badam et al. [10] has shown that assuming thermal equilibrium at the interface and $\alpha_{e}=\alpha_{c}$ requires altering the accommodation coefficient by an order of magnitude in order to match experimental data. Additional assumptions include thermal equilibrium of the interface, neglect of vapor density etc.

In December 2017, I submitted a graduate student grant proposal to NASA under the Physical Sciences Informatics (PSI) program and the proposal has recently been awarded. The project aims at an investigation of the validity of the above described assumptions and the development of a new technique to simultaneously determine both evaporation and condensation coefficients. This work will utilize data collected in previous fluid physics experiments conducted on the International Space Station and will serve as a postdoctoral project. 


\section{Chapter 7}

\section{Photogallery Entries}

The material contained in this chapter was previously published in the Journal of Heat Transfer. These are short, rapid communication photogallery entries that focus on various unique results obtained from neutron imaging. See Appendix A for documentation of permission to republish this material. The first entry is the ability to use neutron imaging as a non-destructive visualization tool to probe inside opaque metallic containers at cryogenic temperatures. The second and third entries provide insight into the contact angle and wettability of liquid hydrogen on Al 6061 and SS 316. The fourth entry focuses on a neutron attenuation analysis to probe adsorbed liquid films of hydrogen whose thicknesses are lower than the imaging resolution. 

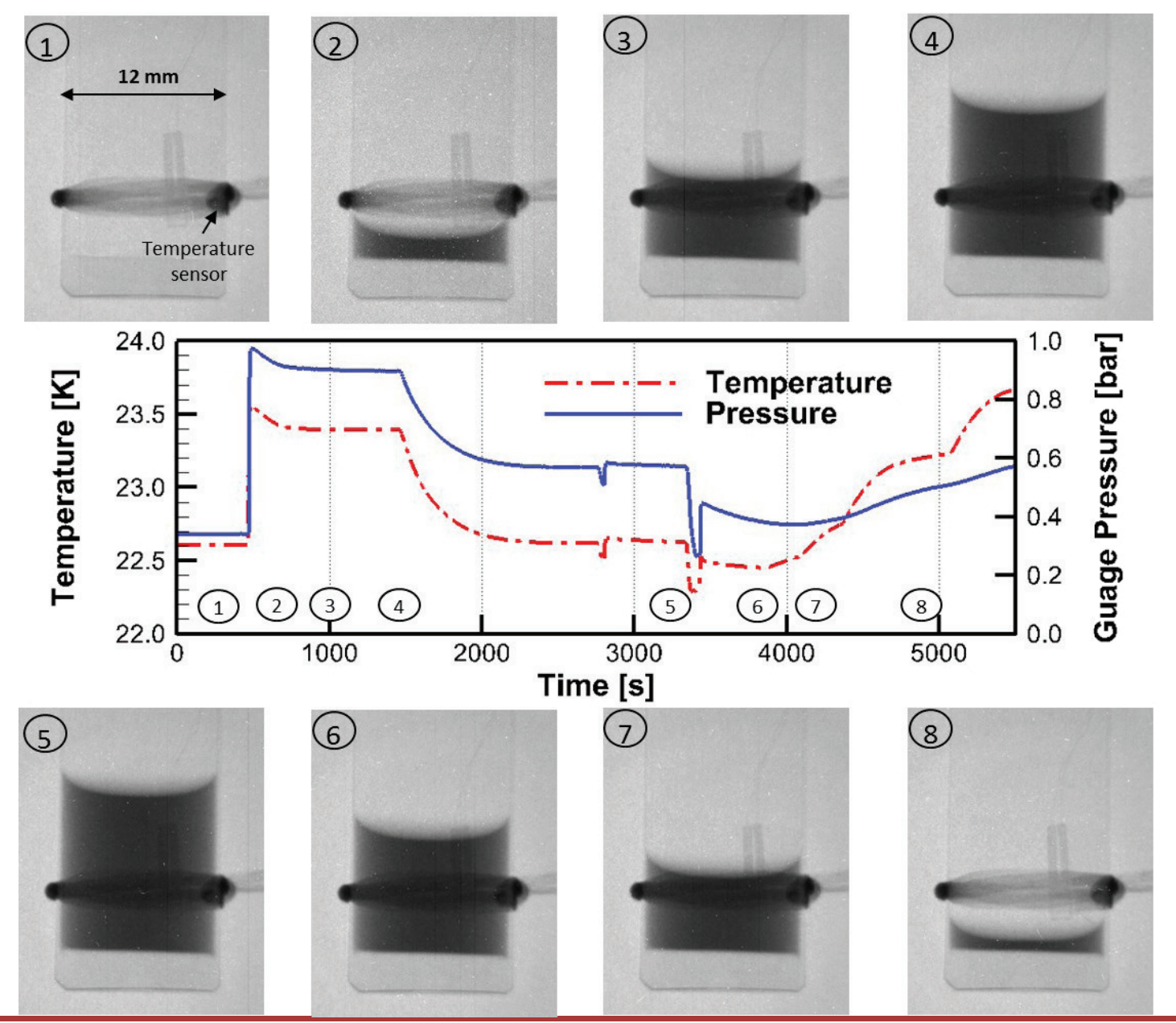

Neutron Radiography of Condensation and Evaporation of Hydrogen in a Cryogenic Condition

Kishan Bellur, Ezequiel Medici, Jeffrey Allen, \& Chang Kyoung Choi, Michigan Technological University, Houghton, MI 49931 Jimes Hermanson \& Arun Tamilarasan, University of Washington, Seattle, WA 98195

Daniel Hussey, David Jacobson, \& Juscelino B. Leao, National Institute of Standards and Technology, Gaithersburg, MD 20899 John McQuillen, NASA Glenn Research Center at Lewis Field, Cleveland, OH 44135

The condensation and evaporation of hydrogen under cryogenic conditions is visualized by using neutron imaging at the BT-2 Beam Facility at the National Institute of Standards and Technology (NIST). The condensation and evaporation are controlled by adjusting temperature $(20 \mathrm{~K} \sim 23 \mathrm{~K})$ and pressure $(1.3 \sim 1.95$ bar absolute). The hydrogen contained in the aluminum test cell inside the cryostat has a large attenuation coefficient due to its large scattering cross section. The high sensitivity of neutron radiography to hydrogen allows the visualization of a meniscus and a contact line of evaporating hydrogenated cryogenic propellants. The graphic represents the temperature, pressure and corresponding images of liquid hydrogen in the test cell. The test cell is made of Aluminum 6061 with an inner diameter of $12 \mathrm{~mm}$. The captured images are then median filtered and post-processed in order to find the volume of liquid hydrogen in the test cell as a function of time. The condensation/evaporation rates obtained from neutron imaging along with corresponding temperature and pressure are used to validate the evaporation model being developed by the authors.

These experiments were conducted at the NIST Center for Neutron Research in the Neutron Imaging Facility and the relevant work is supported by an Early stage Innovations Grant from NASA's Space Technology Research Grants Program (Grant \# NNX14AB05G).

The material in this page was previously published in Journal of Heat Transfer by Bellur et al. [21]. See Appendix A for documentation of permission to republish this material. 


\section{Neutron Radiography}

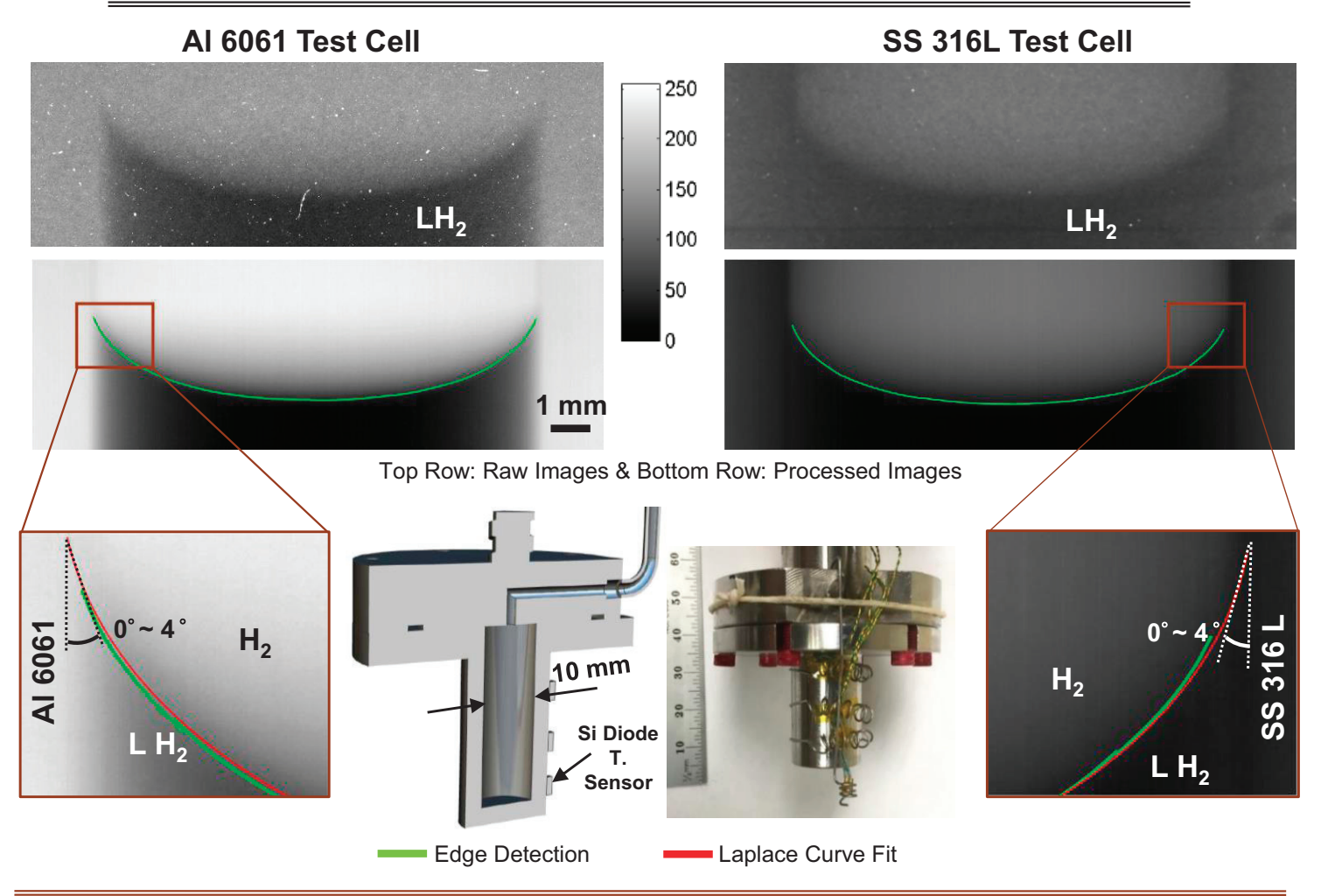

\section{Contact Angle Measurement of Liquid Hydrogen $\left(\mathrm{LH}_{2}\right)$ in Stainless Steel and Aluminum Cells \\ Kishan Bellur, Vinaykumar Konduru, Manan Kulshreshtha, Daanish Tyrewala,}

Ezequiel Medici, Jeffrey S. Allen, \& Chang Kyoung Choi, Michigan Technological University, Houghton, MI

Daniel S. Hussey, David C. Jacobson, \& Juscelino B. Leão, National Institute of Standards and Technology, Gaithersburg, MD John McQuillen, NASA Glenn Research Center at Lewis Field, Cleveland, $\mathrm{OH}$

James Hermanson \& Arun Tamilarasan, University of Washington, Seattle, WA

One of the key limitations to long-term space missions is to avoid propellant boil-off in a microgravity space environment. Even with the use of active and passive controls of propellants, boil off is inevitable. Long-term CFD simulations on propellant behaviors depend on evaporation/condensation coefficients (known as accommodation coefficients) which are in turn dependent upon the wetting characteristics. Phase change experiments were conducted in the BT-2 neutron imaging facility at the National Institute of Standards and Technology (NIST) by introducing vapor $\mathrm{H}_{2}$

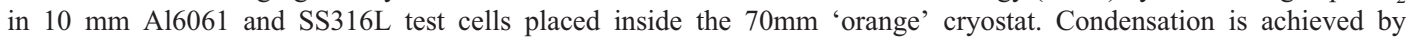
lowering the cryostat temperature below the saturation point and vice versa for evaporation. The high neutron crosssection of liquid $\mathrm{H}_{2}$ in comparison to both the vapor and the test cell materials allows for visualization of a distinct liquid-vapor interface. Multiple images are stacked to increase the signal-to-noise ratio and the meniscus edge is obtained by detecting the pixels with largest gradients in intensities at the liquid meniscus. The contact angle is obtained by curve fitting of the Young-Laplace equation to the detected meniscus. The contact angle for A16061 and SS316 is found to be between $0^{\circ}$ and $4^{\circ}$. The uncertainty arises from edge detection, magnification, and resolution limits of the neutron imaging setup. The test was conducted at a saturation temperature of $21 \mathrm{~K}(1.215 \mathrm{bar})$. The results from the neutron experiments will be then used in conjunction with FEA thermal models and kinetic phase change models to extract accommodation coefficients.

Acknowledgement: This work is supported by an Early Stage Innovations Grant from NASA's Space Technology Research Grants Program (Grant \# NNX14AB05G).

The material in this page was previously published in Journal of Heat Transfer by Bellur et al. [22]. See Appendix A for documentation of permission to republish this material. 


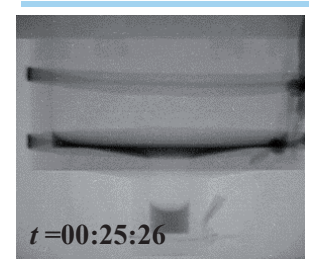

Evaporation
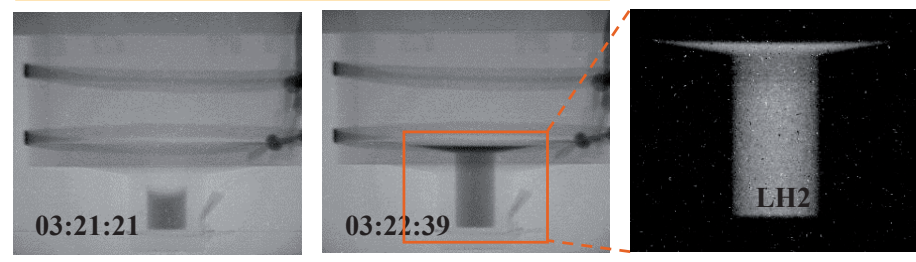

Converted Image

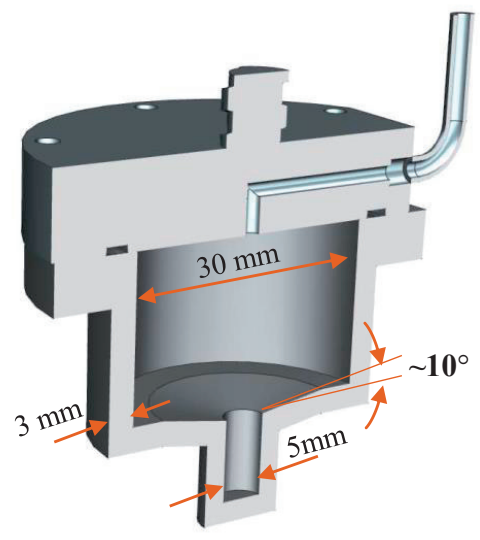

Test Cell Geometry
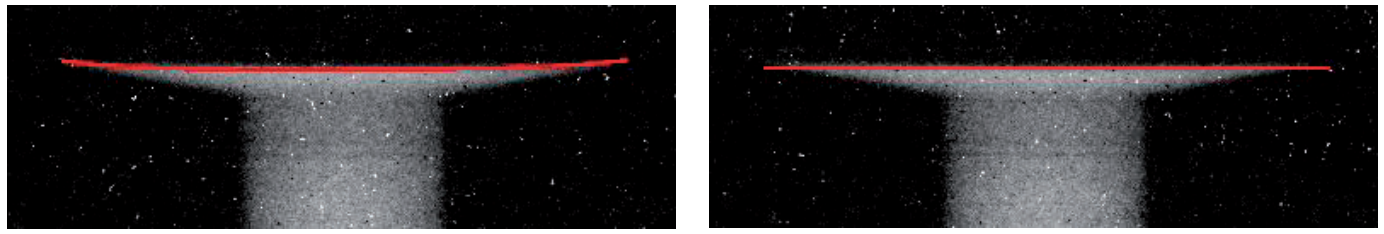

Laplace curve with contact angles of $2^{\circ}$ (Left) and $10^{\circ}$ (Right)

\section{Examining Liquid Hydrogen Wettability Using Neutron Imaging}

Vinaykumar Konduru, Kishan Bellur, Ezequiel F. Médici, Jeffrey S. Allen, Chang Kyoung Choi*,

Michigan Technological University, Houghton, MI 49931

Daniel S. Hussey, David Jacobson, Juscelino B. Leão, National Institute of Standards and Technology, Gaithersburg, MD 20899 John McQuillen, NASA Glenn Research Center at Lewis Field, Cleveland, OH 44135 James C. Hermanson, University of Washington, Seattle, WA 98195

The control of propellant boil-off is essential in long-term space missions. However, a clear understanding of propellant cryogenic condensation/evaporation in microgravity is lacking. One of the key factors in designing such systems is the location of liquid surfaces and the relation to wettability. The BT-2 Neutron Imaging Facility located at the National Institute of Standards and Technology (NIST), Gaithersburg, MD, is used to image evaporation and condensation of hydrogenated propellants inside of an aluminum 6061 container. Liquid hydrogen has larger neutron cross-section area than the aluminum, allowing the visualization of the liquid-vapor interface. The test cell has a conical section that enables determination of a contact angle with enhanced accuracy. If the contact angle is equal to the angle of the cone, a flat liquidvapor interface is expected. The test cell has the cone angle of $10^{\circ}$ and a flat interface was not observed. Using the Laplace-Young equation to fit the interface, the contact angle for hydrogen and aluminum was between $0^{\circ}$ and $4^{\circ}$. The theoretical Laplace curves with contact angles of $2^{\circ}$ and $10^{\circ}$ are plotted on the liquid-vapor interface. The of $2^{\circ}$ curve is a closer fit as compared to the $10^{\circ}$ curve. The uncertainty arises from resolution limits of the neutron imaging setup and edge detection. More details on the neutron imaging mechanism and relevant physics can be found from the authors' other publication of Cryogenics, 74, pp131-137, 2016: doi:10.1016/j.cryogenics.2015.10.016.

Acknowledgement: This work is supported by an Early Stage Innovations Grant from NASA's Space Technology Research Grants Program (Grant \# NNX14AB05G).

The material in this page was previously published in Journal of Heat Transfer by Konduru et al. 77]. See Appendix A for documentation of permission to republish this material. 


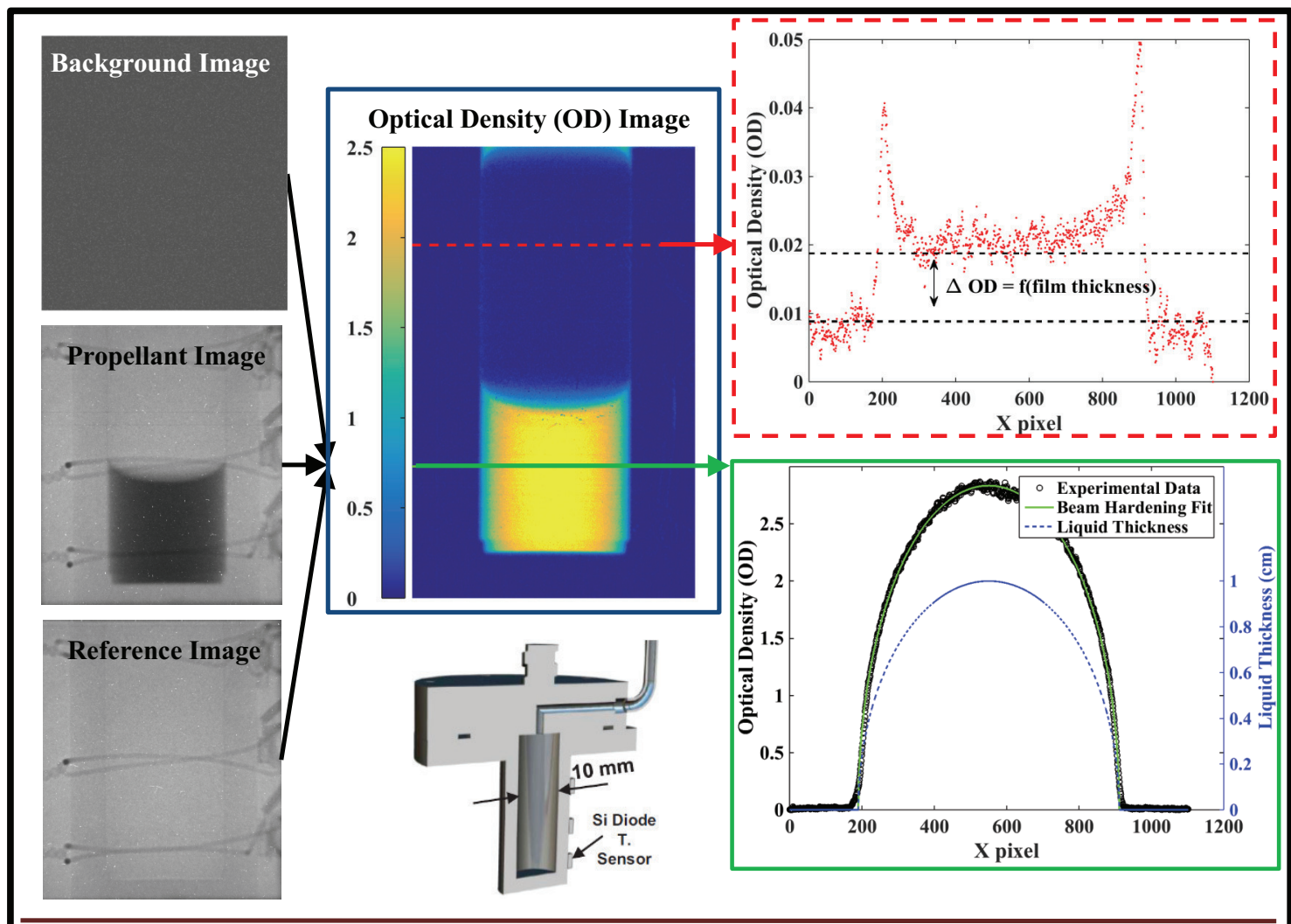

\section{Neutron attenuation analysis of cryogenic propellants}

Kishan Bellur ${ }^{\mathrm{a}}$, Daniel Hussey ${ }^{\mathrm{b}}$, David Jacobson ${ }^{\mathrm{b}}$, Jacob Lamana ${ }^{\mathrm{b}}$, Ezequiel Medici ${ }^{\mathrm{a}}$, James Hermanson $^{c}$, Jeffrey S. Allen ${ }^{\mathrm{a}}$, and Chang Kyoung Choi ${ }^{\mathrm{a},{ }^{*} \text {. }}$

aMichigan Technological University, 49931

bNational Institute of Standards and Technology, 20899

${ }^{c}$ University of Washington, 98195

Neutron imaging enables direct visualization of evaporation and condensation of cryogenic propellants in metal containers such as aluminum and stainless steel. CFD models of propellant behaviors inside the large tanks have shown that a thin liquid film is formed along the interior surface, but this had not been verified experimentally. In the present study, neutron imaging is used to study evaporation and condensation rates of liquid methane inside a cylindrical $10 \mathrm{~mm}, \mathrm{Al} 6061$ cell. The liquid meniscus is clearly shown, but the spatial resolution is insufficient to directly image thin liquid films that may be on the interior surface. Optical density (neutron attenuation) analysis enables quantitative measurements of these liquid films. An optical density image is formed by removing the background noise and normalizing the liquid image with that of the empty cell. Optical densities are then transformed into a liquid transmission thickness using the Beer-Lambert law. This technique enables measurement of film thicknesses smaller than the spatial resolution of the imaging system. The above graphic shows an optical density image during condensation of methane and the corresponding horizontal scan which suggests that a $11 \mu \mathrm{m}$ film exists on the wall. The images indicate that methane undergoes film-wise condensation and is perfectly wetting to aluminum.

These experiments were conducted at the NIST Center for Neutron Research in the Neutron Imaging Facility and the relevant work is supported by an Early stage Innovations Grant from NASA’s Space Technology Research Grants Program (Grant \# NNX14AB05G).

The material in this page was previously published in Journal of Heat Transfer by Bellur et al. [24]. See Appendix A for documentation of permission to republish this material. 



\section{References}

[1] Vladimir S. Ajaev. Interfacial Fluid Mechanics. Springer US, 2012. doi: 10. 1007/978-1-4614-1341-7.

[2] Yiğit Akkuş and Zafer Dursunkaya. A new approach to thin film evaporation modeling. International Journal of Heat and Mass Transfer, 101:742-748, oct 2016. doi: 10.1016/j.ijheatmasstransfer.2016.05.091.

[3] Samantha J. Alberts, Praveen Srikanth, Steven H. Collicott, and Stephen D. Heister. Experiment design for measuring accommodation coefficients for modeling of long-duration spaceflight cryogenic propellants. In 13th International Energy Conversion Engineering Conference. American Institute of Aeronautics and Astronautics, jul 2015. doi: 10.2514/6.2015-4246.

[4] Samantha J. Alberts, Praveen Srikanth, Steven H. Collicott, and Stephen D. Heister. Numerical approach to measure accommodation coefficients for longduration spaceflight cryogenic propellants. In 52nd AIAA/SAE/ASEE Joint 
Propulsion Conference. American Institute of Aeronautics and Astronautics, jul 2016. doi: 10.2514/6.2016-4675.

[5] T. Alty. The reflection of vapour molecules at a liquid surface. Proceedings of the Royal Society A: Mathematical, Physical and Engineering Sciences, 131 (818):554-564, jun 1931. doi: 10.1098/rspa.1931.0072.

[6] T. Alty and C. A. Mackay. The accommodation coefficient and the evaporation coefficient of water. Proceedings of the Royal Society A: Mathematical, Physical and Engineering Sciences, 149(866):104-116, mar 1935. doi: 10.1098/rspa.1935. 0050.

[7] T. Alty and C. A. Mackay. The accommodation coefficient and the evaporation coefficient of water. Proceedings of the Royal Society A: Mathematical, Physical and Engineering Sciences, 149(866):104-116, mar 1935. doi: 10.1098/rspa.1935. 0050.

[8] T. Alty and F. H. Nicoll. THE INTERCHANGE OF MOLECULES BETWEEN a LIQUID AND ITS VAPOR. Canadian Journal of Research, 4(6): 547-558, jun 1931. doi: 10.1139/cjr31-041.

[9] V D Arp. Thermophysical properties of helium-4 from 0.8 to $1500 \mathrm{k}$ with pressures to $2000 \mathrm{MPa}$. Technical report, 1998.

[10] V.K. Badam, V. Kumar, F. Durst, and K. Danov. Experimental and theoretical investigations on interfacial temperature jumps during evaporation. 
Experimental Thermal and Fluid Science, 32(1):276-292, oct 2007. doi: 10.1016/j.expthermflusci.2007.04.006.

[11] G.T. Barnes. The effects of monolayers on the evaporation of liquids. Advances in Colloid and Interface Science, 25:89-200, 1986. doi: 10.1016/0001-8686(86) 80004-5.

[12] Jonathan Barrett and Charles Clement. Kinetic evaporation and condensation rates and their coefficients. Journal of Colloid and Interface Science, 150(2): 352-364, may 1992. doi: 10.1016/0021-9797(92)90205-z.

[13] S Barsi, Mohammed Kassemi, C Panzarella, and J.I.D. Alexander. A tank selfpressurization experiment using a model fluid in normal gravity. In 43rd AIAA Aerospace Sciences Meeting and Exhibit. American Institute of Aeronautics and Astronautics, jan 2005. doi: 10.2514/6.2005-1143.

[14] Stephen Barsi and Mohammad Kassemi. Numerical simulations of the zero boiloff tank experiment. In 46th AIAA Aerospace Sciences Meeting and Exhibit. American Institute of Aeronautics and Astronautics, jan 2008. doi: 10.2514/6. 2008-810.

[15] Stephen Barsi and Mohammad Kassemi. Investigation of tank pressurization and pressure control - part II: Numerical modeling. Journal of Thermal Science and Engineering Applications, 5(4):041006, sep 2013. doi: 10.1115/1.4023892. 
[16] D. Bayer, U. Gross, and K. Raed. A new facility for the experimental investigation on nano heat transfer between gas molecules and ceramic surfaces. In 32nd International Thermal Conductivity Conference, 2014. doi: $10.5703 / 1288284315549$.

[17] A. Bazylak. Liquid water visualization in PEM fuel cells: A review. International Journal of Hydrogen Energy, 34(9):3845-3857, may 2009. doi: 10.1016/j.ijhydene.2009.02.084.

[18] K Bellur. An assessment of the validity of the kinetic model for liquid-vapor phase change by examining cryogenic propellants. Master's thesis, Michigan Technological University, 2016.

[19] K. Bellur, E.F. Médici, M. Kulshreshtha, V. Konduru, D. Tyrewala, A. Tamilarasan, J. McQuillen, J.B. Leão, D.S. Hussey, D.L. Jacobson, J. Scherschligt, J.C. Hermanson, C.K. Choi, and J.S. Allen. A new experiment for investigating evaporation and condensation of cryogenic propellants. Cryogenics, 74:131-137, mar 2016. doi: 10.1016/j.cryogenics.2015.10.016.

[20] K. Bellur, E.F. Médici, J.C. Hermanson, C.K. Choi, and J.S. Allen. Determining solid-fluid interface temperature distribution during phase change of cryogenic propellants using transient thermal modeling. Cryogenics, 91:103-111, apr 2018. doi: 10.1016/j.cryogenics.2018.02.009. 
[21] Kishan Bellur, Ezequiel Medici, Jeffrey Allen, Chang Kyoung Choi, Jimes Hermanson, Arun Tamilarasan, Daniel Hussey, David Jacobson, Juscelino B. Leao, and John McQuillen. Neutron radiography of condensation and evaporation of hydrogen in a cryogenic condition. Journal of Heat Transfer, 137(8):080901, jun 2015. doi: 10.1115/1.4030442.

[22] Kishan Bellur, Vinaykumar Konduru, Manan Kulshrestha, Daanish Tyrewala, Ezequiel Medici, Jeffrey S. Allen, Chang Kyoung Choi, Daniel S. Hussey, David C. Jacobson, Juscelino B. Leão, John McQuillen, James Hermanson, and Arun Tamilarasan. Contact angle measurement of liquid hydrogen (LH2) in stainless steel and aluminum cells. Journal of Heat Transfer, 138(2):020904, jan 2016. doi: 10.1115/1.4032232.

[23] Kishan Bellur, Vinaykumar Konduru, Ezequiel F. Medici, Daniel S. Hussey, David L. Jacobson, Jacob M. LaManna, Jeffrey S. Allen, and Chang Kyoung Choi. VISUALIZATION OF THE EVAPORATION AND CONDENSATION PHENOMENA IN CRYOGENIC PROPELLANTS. Journal of Flow Visualization and Image Processing, 23(1-2):137-156, 2016. doi: 10.1615/ jflowvisimageproc. 2017020115 .

[24] Kishan Bellur, Daniel Hussey, David Jacobson, Jacob Lamana, Ezequiel Medici, James Hermanson, Dr. Jeffrey S. Allen, and Chang Kyoung Choi. Neutron attenuation analysis of cryogenic propellants. Journal of Heat Transfer, 140(3): 030904, feb 2018. doi: 10.1115/1.4039169. 
[25] Laxmidhar Biswal, S.K. Som, and Suman Chakraborty. Thin film evaporation in microchannels with slope- and curvature-dependent disjoining pressure. International Journal of Heat and Mass Transfer, 57(1):402-410, jan 2013. doi: 10.1016/j.ijheatmasstransfer.2012.10.022.

[26] J.S. Brenizer. A review of significant advances in neutron imaging from conception to the present. Physics Procedia, 43:10-20, 2013. doi: 10.1016/j.phpro. 2013.03.002.

[27] C. E. Bryson, V. Cazcarra, M. Chouarain, and L. L. Levenson. High-precision measurements of condensation coefficients. results for carbon dioxide and water molecules. Journal of Vacuum Science and Technology, 9(1):557-560, jan 1972. doi: $10.1116 / 1.1316684$.

[28] C. E. Bryson, V. Cazcarra, and L. L. Levenson. Condensation coefficient measurements of h2o, n2o, and CO2. Journal of Vacuum Science and Technology, 11(1):411-416, jan 1974. doi: 10.1116/1.1318639.

[29] G. Burrows. Evaporation at low pressures. Journal of Applied Chemistry, 7(7): 375-384, may 1957. doi: 10.1002/jctb.5010070706.

[30] G Burrows. Molecular Distillation. Oxford University Press, 1960.

[31] G Burrows. Evaporation in an evacuated container. Vacuum, 15(8):389-399, aug 1965. doi: 10.1016/0042-207x(65)90482-3. 
[32] G. Burrows. Notes on some features of high-vacuum distillation. Vacuum, 23 (10):353-358, oct 1973. doi: 10.1016/0042-207x(73)90490-9.

[33] H. K. Cammenga, H. Klinge, and B. E. Rudolph. Untersuchungen ber die verdampfungs-geschwindigkeit von flssigkeiten. Fortschrittsberichte ber Kolloide und Polymere, 55(1):118-123, 1971. ISSN 0071-8017. doi: 10.1007/BF02826279. URL http://dx.doi.org/10.1007/BF02826279.

[34] V. P. Carey, G. Chen, C. Grigoropoulos, M. Kaviany, and A. Majumdar. A review of heat transfer physics. Nanoscale and Microscale Thermophysical Engineering, 12(1):1-60, jan 2008. doi: 10.1080/15567260801917520.

[35] Van P. Carey. Liquid-Vapor Phase-Change Phenomena. CRC Press, 2nd edition, 2007.

[36] M.B. Chadwick, P. Obložinský, M. Herman, N.M. Greene, R.D. McKnight, D.L. Smith, P.G. Young, R.E. MacFarlane, G.M. Hale, S.C. Frankle, A.C. Kahler, T. Kawano, R.C. Little, D.G. Madland, P. Moller, R.D. Mosteller, P.R. Page, P. Talou, H. Trellue, M.C. White, W.B. Wilson, R. Arcilla, C.L. Dunford, S.F. Mughabghab, B. Pritychenko, D. Rochman, A.A. Sonzogni, C.R. Lubitz, T.H. Trumbull, J.P. Weinman, D.A. Brown, D.E. Cullen, D.P. Heinrichs, D.P. McNabb, H. Derrien, M.E. Dunn, N.M. Larson, L.C. Leal, A.D. Carlson, R.C. Block, J.B. Briggs, E.T. Cheng, H.C. Huria, M.L. Zerkle, K.S. Kozier, A. Courcelle, V. Pronyaev, and S.C. van der Marck. ENDF/b-VII.0: Next generation 
evaluated nuclear data library for nuclear science and technology. Nuclear Data Sheets, 107(12):2931-3060, dec 2006. doi: 10.1016/j.nds.2006.11.001.

[37] Sydney Chapman, Thomas George Cowling, and David Burnett. The mathematical theory of non-uniform gases: an account of the kinetic theory of viscosity, thermal conduction and diffusion in gases. Cambridge University Press, 1970.

[38] E. Cheng, G. Mistura, H. C. Lee, M. H. W. Chan, M. W. Cole, C. Carraro, W. F. Saam, and F. Toigo. Wetting transitions of liquid hydrogen films. Physical Review Letters, 70(12):1854-1857, mar 1993. doi: 10.1103/physrevlett.70.1854.

[39] John M Cimbala, Jack S Brenizer, Abel Po-Ya Chuang, Shane Hanna, C Thomas Conroy, A.A El-Ganayni, and David R Riley. Study of a loop heat pipe using neutron radiography. Applied Radiation and Isotopes, 61(4):701-705, oct 2004. doi: 10.1016/j.apradiso.2004.03.104.

[40] Paul Concus. Static menisci in a vertical right circular cylinder. Journal of Fluid Mechanics, 34(03):481, dec 1968. doi: 10.1017/s002211206800203x.

[41] National Research Council. NASA Space Technology Roadmaps and Priorities. National Academies Press, may 2012. doi: 10.17226/13354.

[42] S. DasGupta, J. A. Schonberg, and P. C. Wayner. Investigation of an evaporating extended meniscus based on the augmented young-laplace equation. Journal of Heat Transfer, 115(1):201, 1993. doi: 10.1115/1.2910649. 
[43] S. DasGupta, I. Y. Kim, and P. C. Wayner. Use of the kelvin-clapeyron equation to model an evaporating curved microfilm. Journal of Heat Transfer, 116(4): 1007, 1994. doi: 10.1115/1.2911436.

[44] P. Davidovits, D. R. Worsnop, J. T. Jayne, C. E. Kolb, P. Winkler, A. Vrtala, P. E. Wagner, M. Kulmala, K. E. J. Lehtinen, T. Vesala, and M. Mozurkewich. Mass accommodation coefficient of water vapor on liquid water. Geophysical Research Letters, 31(22), nov 2004. doi: 10.1029/2004gl020835.

[45] Paul Davidovits, Charles E. Kolb, Leah R. Williams, John T. Jayne, and Douglas R. Worsnop. Update 1 of: Mass accommodation and chemical reactions at gas-liquid interfaces. Chemical Reviews, 111(4), apr 2011. doi: $10.1021 / \mathrm{cr} 100360 \mathrm{~b}$.

[46] E.J. Davis. A history and state-of-the-art of accommodation coefficients. Atmospheric Research, 82(3-4):561-578, dec 2006. doi: 10.1016/j.atmosres.2006. 02.013 .

[47] B. V. Derjaguin, S. V. Nerpin, and N. V. Churaev. Effect of film transfer upon evaporation of liquids from capillaries. Bulletin Rilem, 29:93-98, 1965.

[48] J. Dewanckele, T. De Kock, G. Fronteau, H. Derluyn, P. Vontobel, M. Dierick, L. Van Hoorebeke, P. Jacobs, and V. Cnudde. Neutron radiography and x-ray computed tomography for quantifying weathering and water uptake processes 
inside porous limestone used as building material. Materials Characterization, 88:86-99, feb 2014. doi: 10.1016/j.matchar.2013.12.007.

[49] Shi-Yuan Du and Yao-Hua Zhao. New boundary conditions for the evaporating thin-film model in a rectangular micro channel. International Journal of Heat and Mass Transfer, 54(15-16):3694-3701, jul 2011. doi: 10.1016/j. ijheatmasstransfer.2011.02.059.

[50] I E Dzyaloshinskii, E M Lifshitz, and Lev P Pitaevskii. GENERAL THEORY OF VAN DER WAALS' FORCES. Soviet Physics Uspekhi, 4(2):153-176, feb 1961. doi: 10.1070/pu1961v004n02abeh003330.

[51] I.W. Eames, N.J. Marr, and H. Sabir. The evaporation coefficient of water: a review. International Journal of Heat and Mass Transfer, 40(12):2963-2973, aug 1997. doi: 10.1016/s0017-9310(96)00339-0.

[52] Jack Ekin. Experimental Techniques for Low-Temperature Measurements. Oxford University Press, oct 2006. doi: 10.1093/acprof:oso/9780198570547.001. 0001.

[53] David L Fritz. Implementation of a phenomenological evaporation model into a porous network simulation for water management in low temperature fuel cells. PhD thesis, Michigan Technological University, 2012.

[54] Eckart Fuhrmann and Michael E. Dreyer. Heat and mass transfer at a 
free surface with diabatic boundaries in a single-species system under microgravity conditions. Experiments in Fluids, 55(6), jun 2014. doi: 10.1007/ s00348-014-1760-2.

[55] Vai-Meng Ha and Chun-Liang Lai. Theoretical analysis of marangoni instability of an evaporating droplet by energy method. International Journal of Heat and Mass Transfer, 47(17-18):3811-3823, aug 2004. doi: 10.1016/j. ijheatmasstransfer.2004.03.016.

[56] Michael S. Hanchak, Marlin D. Vangsness, Larry W. Byrd, and Jamie S. Ervin. Thin film evaporation of n-octane on silicon: Experiments and theory. International Journal of Heat and Mass Transfer, 75:196-206, aug 2014. doi: 10.1016/j.ijheatmasstransfer.2014.03.063.

[57] B.A. Hands and V.D. Arp. A correlation of thermal conductivity data for helium. Cryogenics, 21(12):697-703, dec 1981. doi: 10.1016/0011-2275(81) 90211-3.

[58] P. Von Der Hardt and H. Rttger, editors. Neutron Radiography Handbook. Springer Netherlands, 1981. doi: 10.1007/978-94-009-8567-4.

[59] K. Hartmut and K. Ernst. Photographic detection of slowly moving neutrons, January 91940.

[60] Jason Hartwig and John McQuillen. Analysis of screen channel LAD bubble 
point tests in liquid oxygen at elevated temperature. In 42nd AIAA Thermophysics Conference. American Institute of Aeronautics and Astronautics, jun 2011. doi: $10.2514 / 6.2011-3775$.

[61] Jason Hartwig and John McQuillen. Analysis of screen channel LAD bubble point tests in liquid methane at elevated temperature. In 50th AIAA Aerospace Sciences Meeting including the New Horizons Forum and Aerospace Exposition. American Institute of Aeronautics and Astronautics, jan 2012. doi: 10.2514/6. 2012-759.

[62] Jason Hartwig and John McQuillen. Performance gains of propellant management devices for liquid hydrogen depots. In 51st AIAA Aerospace Sciences Meeting including the New Horizons Forum and Aerospace Exposition. American Institute of Aeronautics and Astronautics, jan 2013. doi: $10.2514 / 6.2013-368$.

[63] S. Herminghaus, J. Vorberg, H. Gau, R. Conradt, D. Reinelt, H. Ulmer, P. Leiderer, and M. Przyrembel. Hydrogen and helium films as model systems of wetting. Annalen der Physik, 509(6):425-447, 1997. doi: 10.1002/andp. 19975090602.

[64] H. Hertz. Ueber die verdunstung der flssigkeiten, insbesondere des quecksilbers, im luftleeren raume. Annalen der Physik, 253(10):177-193, 1882. doi: 10.1002/ andp. 18822531002 . 
[65] F. W. Holm and S. P. Goplen. Heat transfer in the meniscus thin-film transition region. Journal of Heat Transfer, 101(3):543, 1979. doi: 10.1115/1.3451025.

[66] R Hołyst, M Litniewski, D Jakubczyk, K Kolwas, M Kolwas, K Kowalski, S Migacz, S Palesa, and M Zientara. Evaporation of freely suspended single droplets: experimental, theoretical and computational simulations. Reports on Progress in Physics, 76(3):034601, feb 2013. doi: 10.1088/0034-4885/76/3/ 034601.

[67] M. Hoorfar and A. W. Neumann. Recent progress in axisymmetric drop shape analysis (ADSA). Advances in Colloid and Interface Science, 121(1-3):25-49, sep 2006. doi: 10.1016/j.cis.2006.06.001.

[68] D. S. Hussey, D. L. Jacobson, M. Arif, K. J. Coakley, and D. F. Vecchia. In situ fuel cell water metrology at the NIST neutron imaging facility. Journal of Fuel Cell Science and Technology, 7(2):021024, 2010. doi: 10.1115/1.3007898.

[69] D.S. Hussey, D.L. Jacobson, M. Arif, P.R. Huffman, R.E. Williams, and J.C. Cook. New neutron imaging facility at the NIST. Nuclear Instruments and Methods in Physics Research Section A: Accelerators, Spectrometers, Detectors and Associated Equipment, 542(1-3):9-15, apr 2005. doi: 10.1016/j.nima.2005. 01.004 .

[70] Jacob Israelachvili. Intermolecular and Surface Forces. Elsevier, 2011. doi: 10.1016/c2009-0-21560-1. 
[71] Anjun Jiao. Modeling of thin film evaporation heat transfer and experimental investigation of miniature heat pipes. PhD thesis, University of MissouriColumbia, 2008.

[72] M. Kang, H.Z. Bilheux, S. Voisin, C.L. Cheng, E. Perfect, J. Horita, and J.M. Warren. Water calibration measurements for neutron radiography: Application to water content quantification in porous media. Nuclear Instruments and Methods in Physics Research Section A: Accelerators, Spectrometers, Detectors and Associated Equipment, 708:24-31, apr 2013. doi: 10.1016/j.nima.2012.12.112.

[73] J. Kapłon, Z. Kawala, and A. Skoczylas. Evaporation rate of a liquid from the surface of a rotating disc in high vacuum. Chemical Engineering Science, 41(3): 519-522, 1986. doi: 10.1016/0009-2509(86)87033-6.

[74] K. Kihm, E. Kirchoff, M. Golden, J. Rosenfeld, S. Rawal, D. Pratt, A. Swanson, H. Bilheux, L. Walker, S. Voisin, D.S. Hussey, and D.L. Jacobson. Neutron imaging of alkali metal heat pipes. Physics Procedia, 43:323-330, 2013. doi: 10.1016/j.phpro.2013.03.038.

[75] M. Knudsen. Maximum rate of vaporization of mercury. Ann. Phys, 47:697-705, 1915.

[76] C. E. Kolb, R. A. Cox, J. P. D. Abbatt, M. Ammann, E. J. Davis, D. J. Donaldson, B. C. Garrett, C. George, P. T. Griffiths, D. R. Hanson, M. Kulmala, G. McFiggans, U. Pschl, I. Riipinen, M. J. Rossi, Y. Rudich, P. E. 
Wagner, P. M. Winkler, D. R. Worsnop, and C. D. O' Dowd. An overview of current issues in the uptake of atmospheric trace gases by aerosols and clouds. Atmospheric Chemistry and Physics, 10(21):10561-10605, nov 2010. doi: $10.5194 /$ acp-10-10561-2010.

[77] Vinaykumar Konduru, Kishan Bellur, Ezequiel F. Médici, Jeffrey S. Allen, Chang Kyoung Choi, Daniel S. Hussey, David Jacobson, Juscelino B. Leão, John McQuillen, and James C. Hermanson. Examining liquid hydrogen wettability using neutron imaging. Journal of Heat Transfer, 138(8):080901, jul 2016. doi: 10.1115/1.4033822.

[78] Sonja Körner. Digital image processing in neutron radiography. PhD thesis, Technische Univ. Wien, 2000.

[79] Rolf Krahl, Jens Gerstmann, Philipp Behruzi, Eberhard Bnsch, and Michael E. Dreyer. Dependency of the apparent contact angle on nonisothermal conditions. Physics of Fluids, 20(4):042101, apr 2008. doi: 10.1063/1.2899641.

[80] Denis Kramer, Jianbo Zhang, Ryoichi Shimoi, Eberhard Lehmann, Alexander Wokaun, Kazuhiko Shinohara, and Gnther G. Scherer. In situ diagnostic of two-phase flow phenomena in polymer electrolyte fuel cells by neutron imaging. Electrochimica Acta, 50(13):2603-2614, apr 2005. doi: 10.1016/j.electacta.2004. 11.005 . 
[81] A.P. Kryukov and V.Yu. Levashov. About evaporation-condensation coefficients on the vapor-liquid interface of high thermal conductivity matters. International Journal of Heat and Mass Transfer, 54(13-14):3042-3048, jun 2011. doi: 10.1016/j.ijheatmasstransfer.2011.02.042.

[82] Nikolai Kulev and Michael Dreyer. Drop tower experiments on non-isothermal reorientation of cryogenic liquids. Microgravity Science and Technology, 22(4): 463-474, sep 2010. doi: 10.1007/s12217-010-9237-2.

[83] J. W. Leachman, R. T Jacobsen, S. G. Penoncello, and E. W. Lemmon. Fundamental equations of state for parahydrogen, normal hydrogen, and orthohydrogen. Journal of Physical and Chemical Reference Data, 38(3):721-748, sep 2009. doi: $10.1063 / 1.3160306$.

[84] Sandra L. Lednovich and John B. Fenn. Absolute evaporation rates for some polar and nonpolar liquids. AIChE Journal, 23(4):454-459, jul 1977. doi: 10. 1002/aic.690230408.

[85] Zhi Liang, Thierry Biben, and Pawel Keblinski. Molecular simulation of steadystate evaporation and condensation: Validity of the schrage relationships. International Journal of Heat and Mass Transfer, 114:105-114, nov 2017. doi: 10.1016/j.ijheatmasstransfer.2017.06.025.

[86] PE Liley and PD Desai. Thermophysical Properties of Refrigerants:(inch-pound Edition). ASHRAE, 1993. 
[87] Jer $\mathrm{Ru}$ Maa. The role of interfaces in heat transfer processes. Advances in Colloid and Interface Science, 18(3-4):227-280, jan 1983. doi: 10.1016/ 0001-8686(83)87003-1.

[88] Douglas Mann. Lng materials and fluids. National Bureau of Standards, Boulder, Colo. 80302. 1977, Sections paged separately(Book)., 1977.

[89] R. Marek and J. Straub. Analysis of the evaporation coefficient and the condensation coefficient of water. International Journal of Heat and Mass Transfer, 44(1):39-53, jan 2001. doi: 10.1016/s0017-9310(00)00086-7.

[90] E. D. Marquardt, J. P. Le, and Ray Radebaugh. Cryogenic material properties database. In Cryocoolers 11, pages 681-687. Kluwer Academic Publishers, 2002. doi: $10.1007 / 0-306-47112-4 \_84$.

[91] Robert D McCarty and Vincent D Arp. A new wide range equation of state for helium. In Advances in Cryogenic Engineering, pages 1465-1475. Springer, 1990.

[92] Robert D McCarty, J Hord, and HM Roder. Selected properties of hydrogen (engineering design data). final report. Technical report, National Engineering Lab.(NBS), Boulder, CO (USA), 1981.

[93] Steven Moosman and G.M Homsy. Evaporating menisci of wetting fluids. Journal of Colloid and Interface Science, 73(1):212-223, jan 1980. doi: 10.1016/0021-9797(80)90138-1. 
[94] S. J. S. Morris. The evaporating meniscus in a channel. Journal of Fluid Mechanics, 494:297-317, nov 2003. doi: 10.1017/s0022112003006153.

[95] Michael Mozurkewich. Aerosol growth and the condensation coefficient for water: A review. Aerosol Science and Technology, 5(2):223-236, jan 1986. doi: $10.1080 / 02786828608959089$.

[96] Masataka Okuyama and Joseph T. Zung. Evaporation-condensation coefficient for small droplets. The Journal of Chemical Physics, 46(5):1580-1585, mar 1967. doi: 10.1063/1.1840906.

[97] Sashidhar S. Panchamgam, Shripad J. Gokhale, Joel L. Plawsky, Sunando DasGupta, and Peter C. Wayner. Experimental determination of the effect of disjoining pressure on shear in the contact line region of a moving evaporating thin film. Journal of Heat Transfer, 127(3):231, 2005. doi: 10.1115/1.1857947.

[98] Sashidhar S. Panchamgam, Arya Chatterjee, Joel L. Plawsky, and Peter C. Wayner. Comprehensive experimental and theoretical study of fluid flow and heat transfer in a microscopic evaporating meniscus in a miniature heat exchanger. International Journal of Heat and Mass Transfer, 51(21-22):53685379, oct 2008. doi: 10.1016/j.ijheatmasstransfer.2008.03.023. 
[99] Charles Panzarella and Mohammad Kassemi. One-dimensional model of evaporation and condensation in the presence of a noncondensable gas with applications to cryogenic fluid storage. International Journal of Heat and Mass Transfer, 52(15-16):3767-3777, jul 2009. doi: 10.1016/j.ijheatmasstransfer.2009.02. 027.

[100] Charles Panzarella, David Plachta, and Mohammad Kassemi. Pressure control of large cryogenic tanks in microgravity. Cryogenics, 44(6-8):475-483, jun 2004. doi: 10.1016/j.cryogenics.2004.03.009.

[101] Charles H. Panzarella and Mohammad Kassemi. On the validity of purely thermodynamic descriptions of two-phase cryogenic fluid storage. Journal of Fluid Mechanics, 484:41-68, jun 2003. doi: 10.1017/s0022112003004002.

[102] Charles H. Panzarella and Mohammad Kassemi. Self-pressurization of large spherical cryogenic tanks in space. Journal of Spacecraft and Rockets, 42(2): 299-308, mar 2005. doi: 10.2514/1.4571.

[103] B. Paul. Compilation of evaporation coefficients. ARS Journal, 32(9):13211328, sep 1962. doi: 10.2514/8.6277.

[104] E. Perfect, C.-L. Cheng, M. Kang, H.Z. Bilheux, J.M. Lamanna, M.J. Gragg, and D.M. Wright. Neutron imaging of hydrogen-rich fluids in geomaterials and engineered porous media: A review. Earth-Science Reviews, 129:120-135, feb 2014. doi: 10.1016/j.earscirev.2013.11.012. 
[105] Aaron H. Persad and Charles A. Ward. Expressions for the evaporation and condensation coefficients in the hertz-knudsen relation. Chemical Reviews, 116 (14):7727-7767, jun 2016. doi: 10.1021/acs.chemrev.5b00511.

[106] Joel L. Plawsky, Manas Ojha, Arya Chatterjee, and Peter C. Wayner. REVIEW OF THE EFFECTS OF SURFACE TOPOGRAPHY, SURFACE CHEMISTRY, AND FLUID PHYSICS ON EVAPORATION AT THE CONTACT LINE. Chemical Engineering Communications, 196(5):658-696, dec 2008. doi: 10.1080/00986440802569679.

[107] Joel L. Plawsky, Arya Chatterjee, and Peter C. Wayner Jr. Modeling conact line dynamics in evaporating menisci. In Excerpt from the Proceedings of the COMSOL Conference 2009 Boston 2009 Comsol Users Conference 8-10 October, Boston, MA USA, 2009.

[108] M Potash and P.C Wayner. Evaporation from a two-dimensional extended meniscus. International Journal of Heat and Mass Transfer, 15(10):1851-1863, oct 1972. doi: 10.1016/0017-9310(72)90058-0.

[109] David M. Pratt and Kevin P. Hallinan. Thermocapillary effects on the wetting characteristics of a heated curved meniscus. Journal of Thermophysics and Heat Transfer, 11(4):519-525, oct 1997. doi: 10.2514/2.6293.

[110] G. Preiss and P. C. Wayner. Evaporation from a capillary tube. Journal of Heat Transfer, 98(2):178, 1976. doi: 10.1115/1.3450515. 
[111] H.R. Pruppacher and J.D. Klett. Microphysics of Clouds and Precipitation. Springer Netherlands, 2010. doi: 10.1007/978-0-306-48100-0.

[112] D. Ross, P. Taborek, and J. E. Rutledge. Wetting behavior ofH2on cesium. Physical Review B, 58(8):R4274-R4276, aug 1998. doi: 10.1103/physrevb.58. r4274.

[113] R. Satija, D.L. Jacobson, M. Arif, and S.A. Werner. In situ neutron imaging technique for evaluation of water management systems in operating PEM fuel cells. Journal of Power Sources, 129(2):238-245, apr 2004. doi: 10.1016/j. jpowsour.2003.11.068.

[114] George R Schmidt. Thermocapillary flow with evaporation and condensation and its effect on liquid retention in low-g fluid acquisition devices. Technical Report TP-3463, NASA, 1994.

[115] J.A. Schonberg, S. DasGupta, and P.C. Wayner. An augmented younglaplace model of an evaporating meniscus in a microchannel with high heat flux. Experimental Thermal and Fluid Science, 10(2):163-170, feb 1995. doi: 10.1016/0894-1777(94)00085-m.

[116] R. W. Schrage. A theoretical study of interphase mass transfer. PhD thesis, Columbia University Press, 1953.

[117] N J Simon, E S Drexler, and R P Reed. Properties of copper and copper alloys at cryogenic temperatures. Technical report, 1992. 
[118] Dhruv Singh, Xiaohui Guo, Alina Alexeenko, Jayathi Y. Murthy, and Timothy S. Fisher. Modeling of subcontinuum thermal transport across semiconductor-gas interfaces. Journal of Applied Physics, 106(2):024314, jul 2009. doi: $10.1063 / 1.3181059$.

[119] A. M. Sinnarwalla, D. J. Alofs, and J. C. Carstens. Measurement of growth rate to determine condensation coefficients for water drops grown on natural cloud nuclei. Journal of the Atmospheric Sciences, 32(3):592-599, mar 1975. doi: 10.1175/1520-0469(1975)032〈0592:mogrtd $\rangle 2.0 . c 0 ; 2$.

[120] P.C. Stephan and C.A. Busse. Analysis of the heat transfer coefficient of grooved heat pipe evaporator walls. International Journal of Heat and Mass Transfer, 35(2):383-391, feb 1992. doi: 10.1016/0017-9310(92)90276-x.

[121] L. W. Swanson and G. C. Herdt. Model of the evaporating meniscus in a capillary tube. Journal of Heat Transfer, 114(2):434, 1992. doi: 10.1115/1. 2911292.

[122] YS Touloukian and CY Ho. Thermophysical properties of selected aerospace materials. part 2. thermophysical properties of seven materials. Technical report, DTIC Document, 1977.

[123] P.C. Wayner, Y.K. Kao, and L.V. LaCroix. The interline heat-transfer coefficient of an evaporating wetting film. International Journal of Heat and Mass Transfer, 19(5):487-492, may 1976. doi: 10.1016/0017-9310(76)90161-7. 
[124] Peter C. Wayner. The effect of interfacial mass transport on flow in thin liquid films. Colloids and Surfaces, 52:71-84, jan 1991. doi: 10.1016/0166-6622(91) 80006-a.

[125] Peter C. Wayner. Intermolecular forces in phase-change heat transfer: 1998 kern award review. AIChE Journal, 45(10):2055-2068, oct 1999. doi: 10.1002/ aic.690451004.

[126] Sang-Kwon Wee, Kenneth D. Kihm, and Kevin P. Hallinan. Effects of the liquid polarity and the wall slip on the heat and mass transport characteristics of the micro-scale evaporating transition film. International Journal of Heat and Mass Transfer, 48(2):265-278, jan 2005. doi: 10.1016/j.ijheatmasstransfer. 2004.08.021.

[127] Sang-Kwon Wee, Kenneth D. Kihm, David M. Pratt, and Jeffrey S. Allen. Microscale heat and mass transport of evaporating thin film of binary mixture. Journal of Thermophysics and Heat Transfer, 20(2):320-326, apr 2006. doi: $10.2514 / 1.15784$.

[128] C. Wilson, B. Borgmeyer, R. A. Winholtz, H. B. Ma, D. L. Jacobson, D. S. Hussey, and M. Arif. Visual observation of oscillating heat pipes using neutron radiography. Journal of Thermophysics and Heat Transfer, 22(3):366-372, jul 2008. doi: $10.2514 / 1.33758$. 
[129] P. M. Winkler, A. Vrtala, R. Rudolf, P. E. Wagner, I. Riipinen, T. Vesala, K. E. J. Lehtinen, Y. Viisanen, and M. Kulmala. Condensation of water vapor: Experimental determination of mass and thermal accommodation coefficients. Journal of Geophysical Research, 111(D19), oct 2006. doi: 10.1029/2006jd007194.

[130] Qingfang Wu and Harris Wong. A slope-dependent disjoining pressure for nonzero contact angles. Journal of Fluid Mechanics, 506:157-185, may 2004. doi: $10.1017 / \mathrm{s} 0022112004008420$. 


\section{Appendix A}

\section{Copyight Permission}

Chapter 2 of this dissertation was originally published as "A new experiment for investigating evaporation and condensation of cryogenic propellants", Cryogenics, Vol 74, 2016, Pages 131-137. A copy of permission for reproduction is shown in figure A.1.

Chapter 3 of this dissertation was originally published as "Visualization of the Evaporation and Condensation Phenomena in Cryogenic Propellants", Journal of Flow Visualization and Image Processing, Vol 23, 2016, Pages 137-156. A copy of permission for reproduction is shown in figure A.2.

Chapter 4 of this dissertation was originally published as "Determining solid-fluid interface temperature distribution during phase change of cryogenic propellants using transient thermal modeling", Cryogenics, Vol 91, 2018, Pages 103-111. A copy of 
permission for reproduction is shown in figure A.7.

Chapter 7 of this dissertation is a compilation of 1 page, rapid publication articles originally published in the Journal of Heat Transfer. The material on page 134 was originally published "Neutron Radiography of Condensation and Evaporation of Hydrogen in a Cryogenic Condition", Journal of Heat Transfer, Vol 137, Issue 8, 2015. A copy of permission for reproduction is shown in figure A.4.

The material on page 135 was originally published "Contact Angle Measurement of Liquid Hydrogen (LH2) in Stainless Steel and Aluminum Cells", Journal of Heat Transfer, Vol 138, Issue 2, 2016. A copy of permission for reproduction is shown in figure A.5.

The material on page 136 was originally published "Examining Liquid Hydrogen Wettability Using Neutron Imaging", Journal of Heat Transfer, Vol 138, Issue 8, 2016. A copy of permission for reproduction is shown in figure A.6.

The material on page 137 was originally published "Neutron attenuation analysis of cryogenic propellants", Journal of Heat Transfer, Vol 140, Issue 3, 2018. A copy of permission for reproduction is shown in figure A.7. 


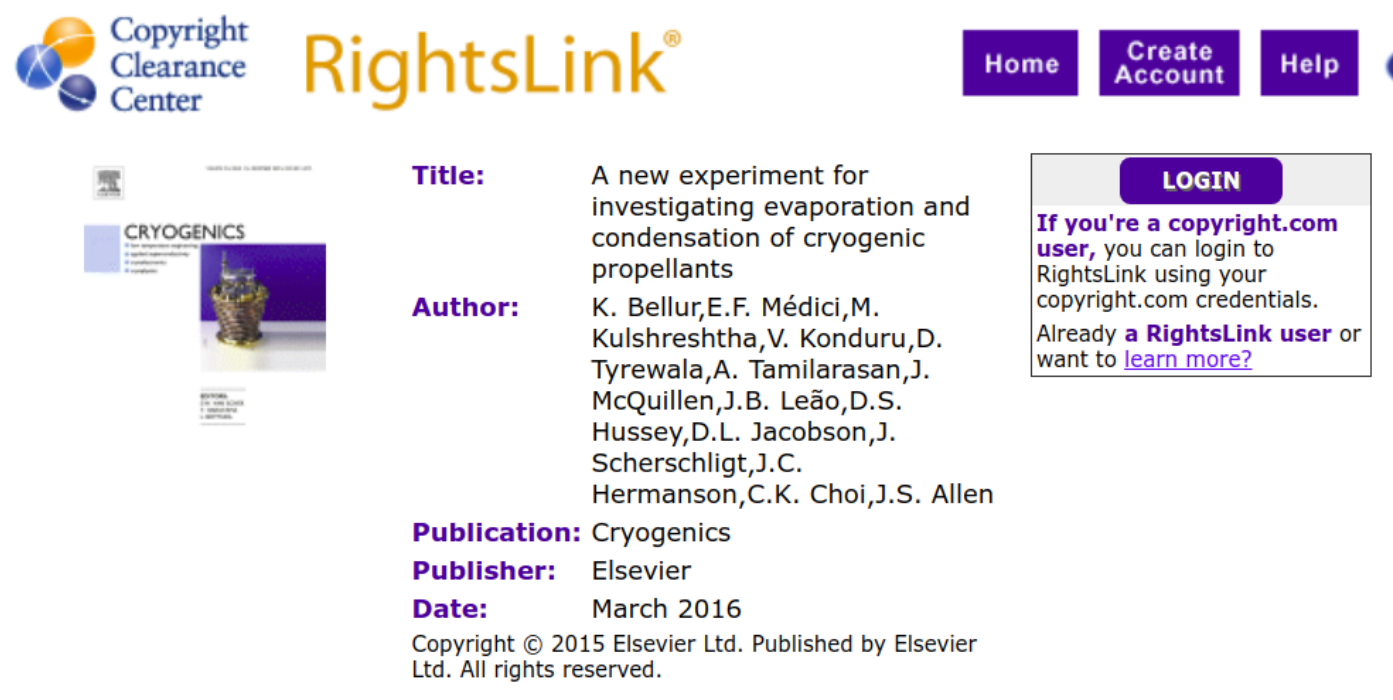

Please note that, as the author of this Elsevier article, you retain the right to include it in a thesis or dissertation, provided it is not published commercially. Permission is not required, but please ensure that you reference the journal as the original source. For more information on this and on your other retained rights, please visit: https://www.elsevier.com/about/our-business/policies/copyright\#Author$\underline{\text { rights }}$

Figure A.1: Permission to reuse material in chapter 2 


\section{hbegell}

New York • Connecticut
50 North Street

Danbury, CT 06810

203-456-6161

journals@begellhouse.com

www.begellhouse.com

June 29, 2018

Kishan Bellur

Michigan Technological University

United States

Re: Permission to Reprint Article

Dear Kishan Bellur,

Permission is hereby granted to reproduce the following article published by Begell House: "VISUALIZATION OF THE EVAPORATION AND CONDENSATION PHENOMENA IN CRYOGENIC PROPELLANTS", by K. Bellur, V. Konduru, E.F. Médici, D.S. Hussey, D.L. Jacobson, J.M. LaManna, J.S. Allen, and C.K. Choi, published in the Journal of Flow Visualization and Image Processing, Volume 23, Issues 1-2, 2016.

Proposed Use: As part of a dissertation titled "A new technique to determine accommodation coefficients of cryogenic propellants"

Publisher: Michigan Technological University, United States

Year of Publication: 2018

As per your email, we hereby grant you permission to reproduce the aforementioned material at no charge subject to the following conditions:

1 - If any part of the material to be used (for example, figures) has appeared in our publication with credit or acknowledgement to another source, permission must also be sought from that source. If such permission is not obtained then that material may not be included in your publication.

2 - Suitable acknowledgement to the source must be made, either as a footnote or in a reference list at the end of your publication, as follows:

"Reprinted from Publication title, Volume number, Author(s), Title of article, Page Nos., Copyright (Year), with permission from Begell House, Inc."

3 - Reproduction of this material is confined to the purpose for which permission is hereby given.

Yours sincerely

Brandon T. Bisceglia

Begell House Inc.

Figure A.2: Permission to reuse material in chapter 3 


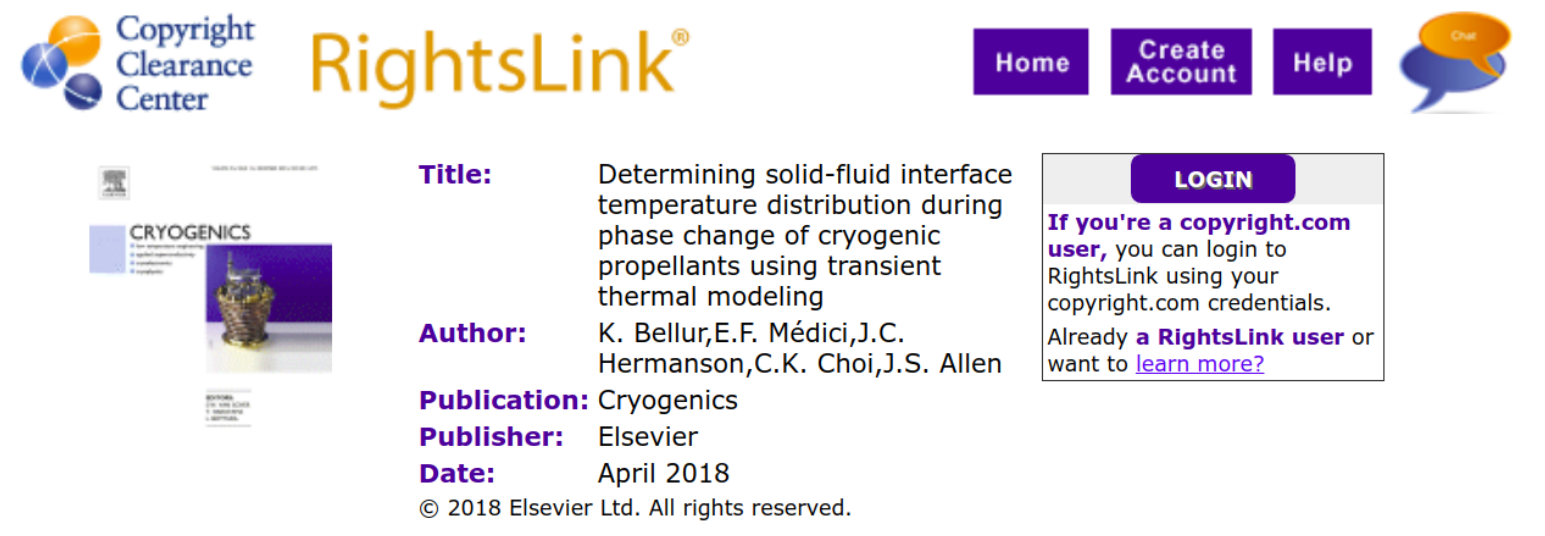

Please note that, as the author of this Elsevier article, you retain the right to include it in a thesis or dissertation, provided it is not published commercially. Permission is not required, but please ensure that you reference the journal as the original source. For more information on this and on your other retained rights, please visit: https://www.elsevier.com/about/our-business/policies/copyright\#Authorrights

Figure A.3: Permission to reuse material in chapter 4 
This is a License Agreement between Kishan S Bellur ("You") and American Society of Mechanical Engineers ASME ("American Society of Mechanical Engineers ASME") provided by Copyright Clearance Center ("CCC"). The license consists of your order details, the terms and conditions provided by American Society of Mechanical Engineers ASME, and the payment terms and conditions.

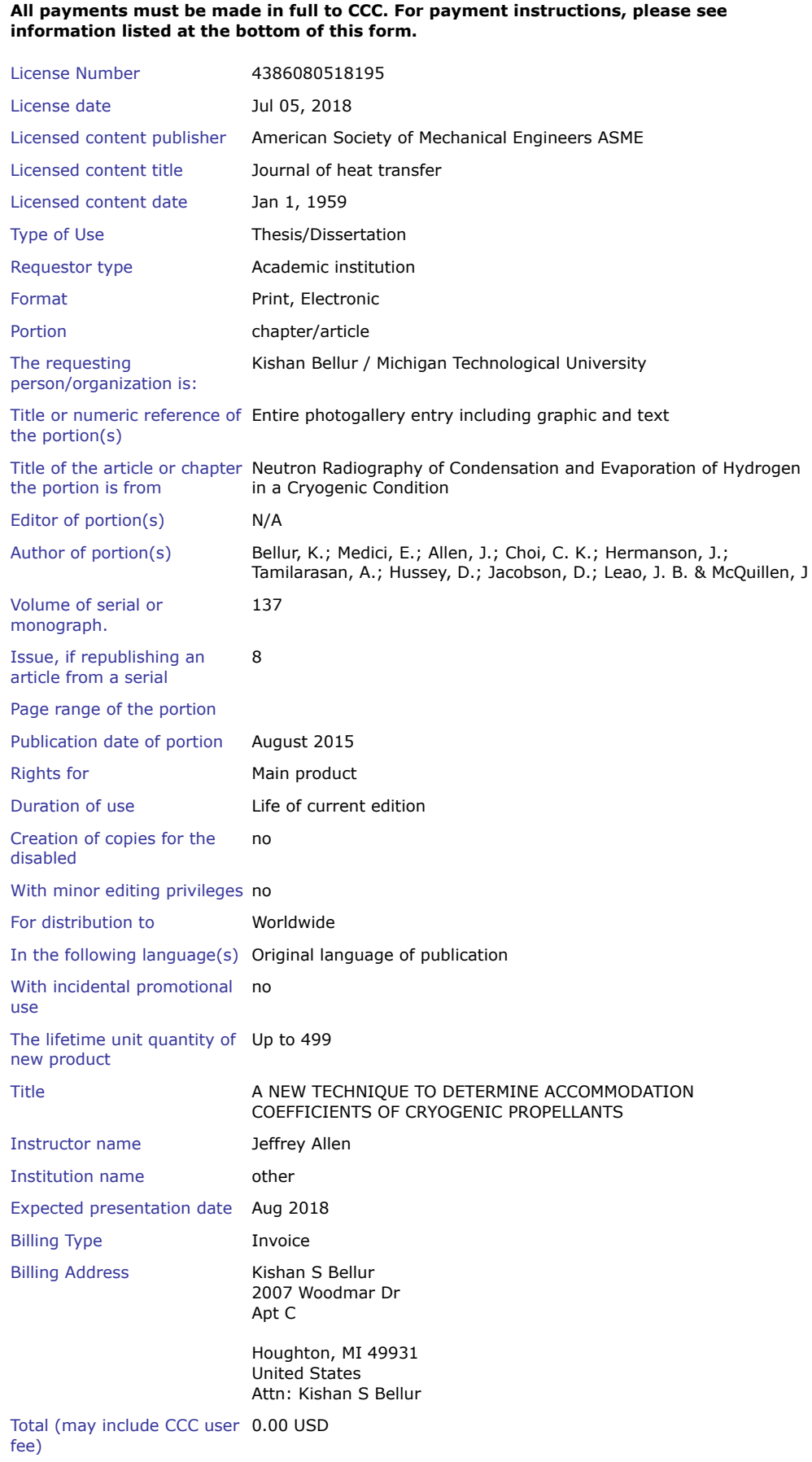

Figure A.4: Permission to reuse material in chapter 7, page 134 


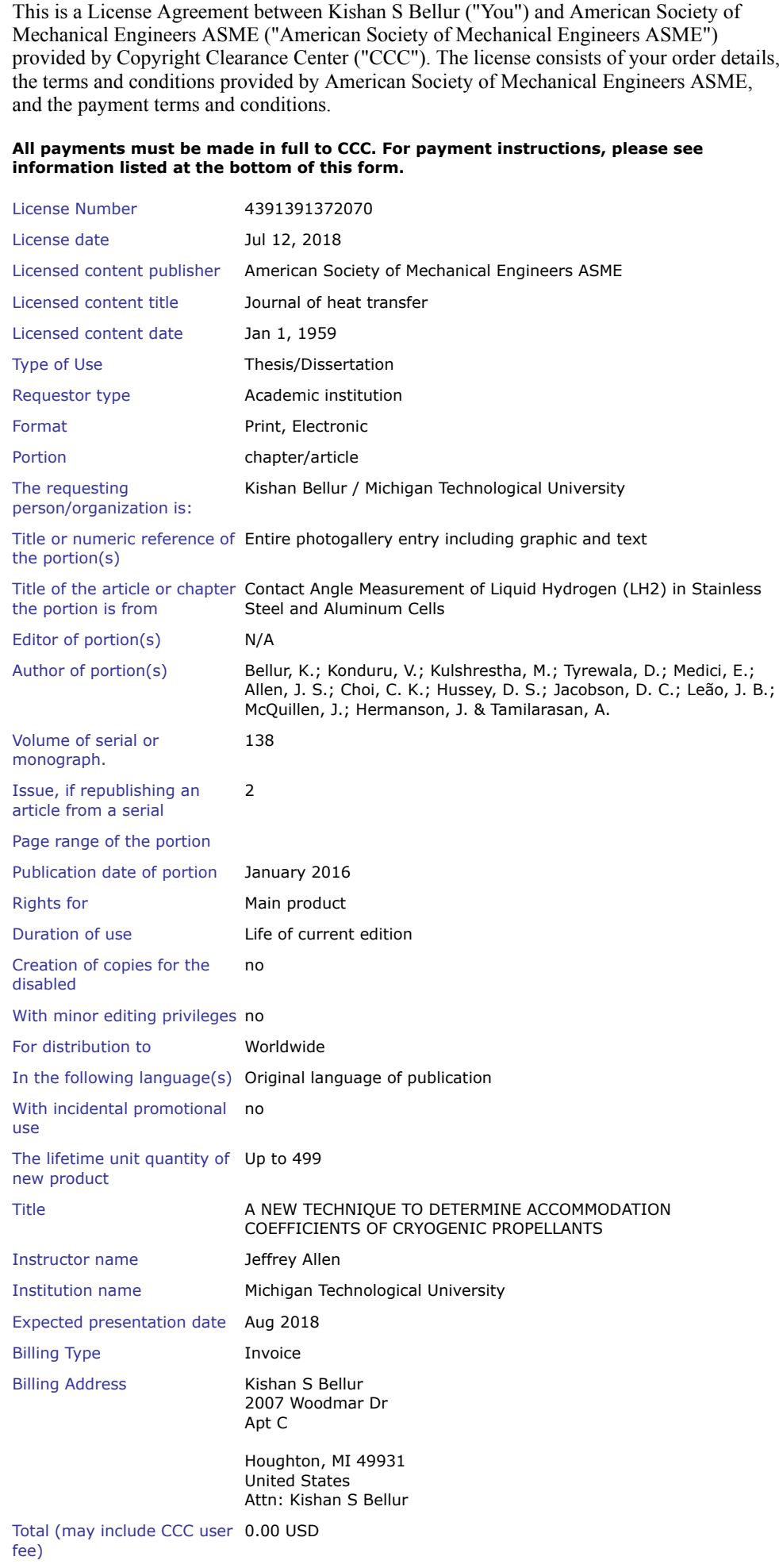

All payments must be made in full to CCC. For payment instructions, please see information listed at the bottom of this form.

Figure A.5: Permission to reuse material in chapter 7, page 135 


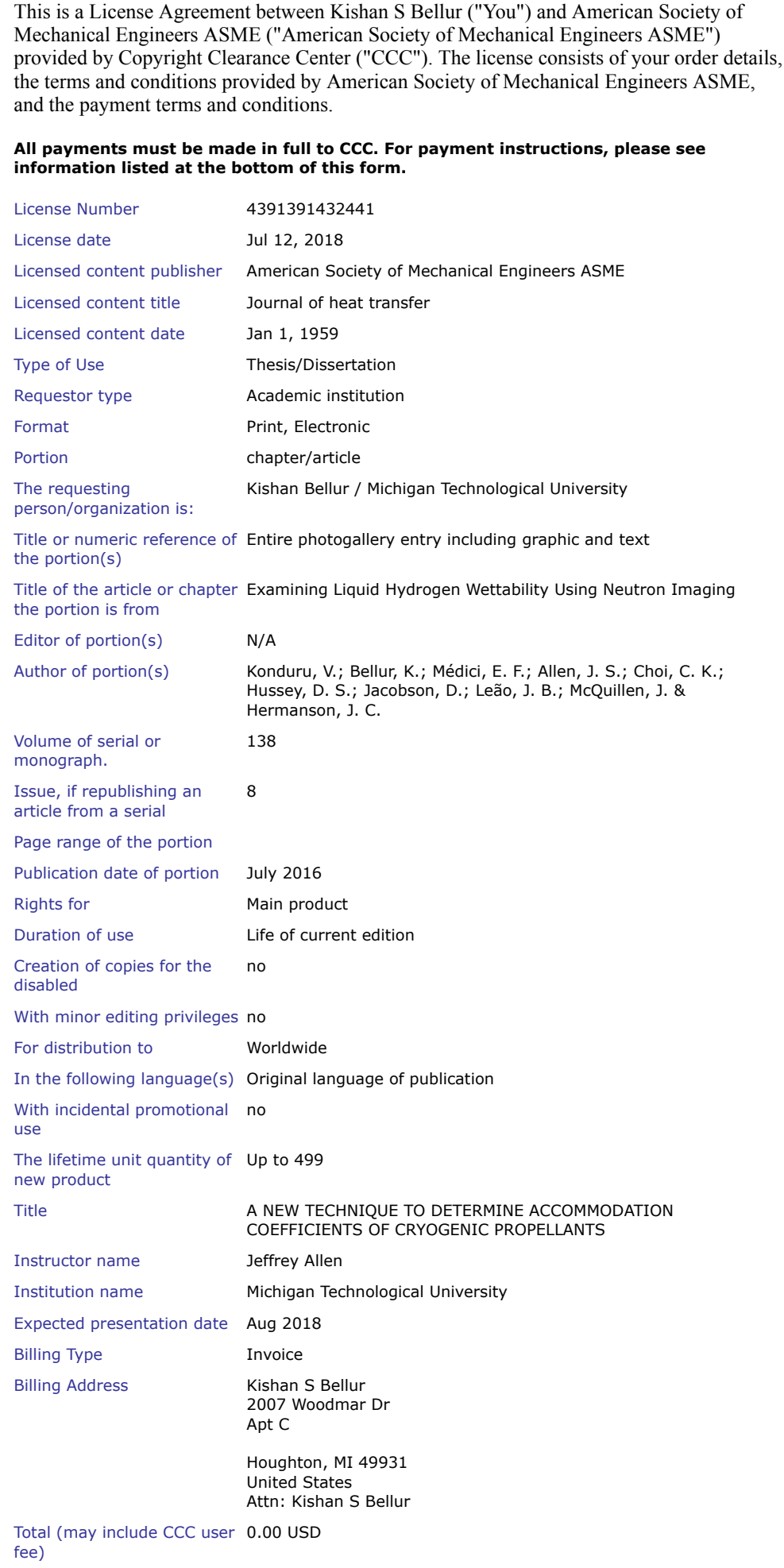

All payments must be made in full to CCC. For payment instructions, please see information listed at the bottom of this form.

License Number 4391391432441

License date Jul 12, 2018

Licensed content publisher American Society of Mechanical Engineers ASME

Licensed content title Journal of heat transfer

Licensed content date Jan 1, 1959

Type of Use

Thesis/Dissertation

Requestor type Academic institution

Format Print, Electronic

Portion chapter/article

The requesting Kishan Bellur / Michigan Technological University

person/organization is:

Title or numeric reference of Entire photogallery entry including graphic and text the portion(s)

Title of the article or chapter Examining Liquid Hydrogen Wettability Using Neutron Imaging the portion is from

Editor of portion(s) N/A

Author of portion(s)

Konduru, V.; Bellur, K.; Médici, E. F.; Allen, J. S.; Choi, C. K.; Hussey, D. S.; Jacobson, D.; Leão, J. B.; McQuillen, J. \& Hermanson, J. C.

Volume of serial or 138

monograph.

Issue, if republishing an 8

article from a serial

Page range of the portion

Publication date of portion July 2016

Rights for

Main product

Duration of use

Life of current edition

Creation of copies for the no

disabled

With minor editing privileges no

For distribution to

Worldwide

In the following language(s) Original language of publication

With incidental promotional no

use

The lifetime unit quantity of Up to 499 new product

Figure A.6: Permission to reuse material in chapter 7, 136 
This is a License Agreement between Kishan S Bellur ("You") and American Society of Mechanical Engineers ASME ("American Society of Mechanical Engineers ASME") provided by Copyright Clearance Center ("CCC"). The license consists of your order details, the terms and conditions provided by American Society of Mechanical Engineers ASME, and the payment terms and conditions.

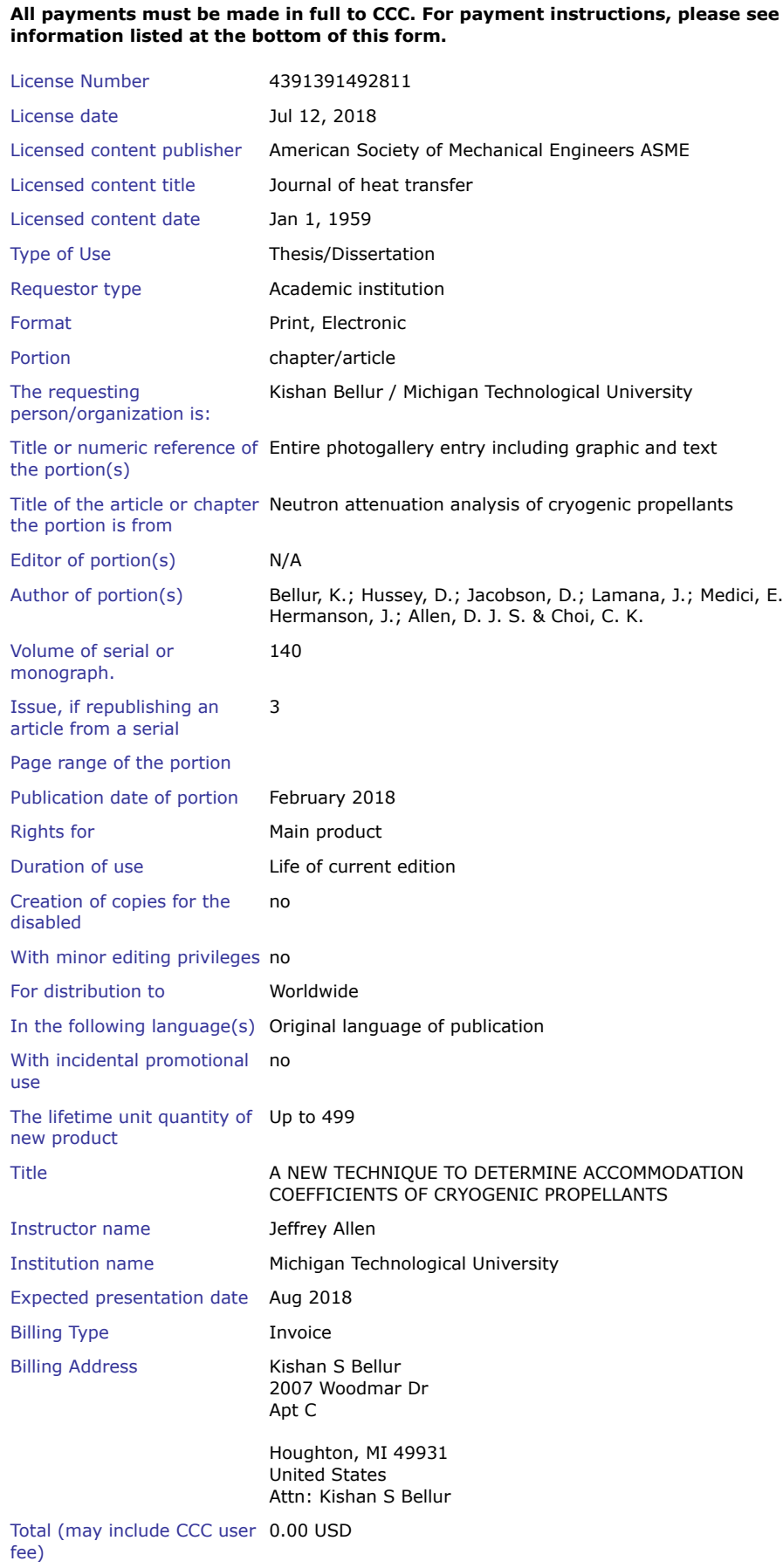

Figure A.7: Permission to reuse material in chapter 7, page 137 



\section{Appendix B}

\section{Code}

\section{B.1 YL_fit.m}

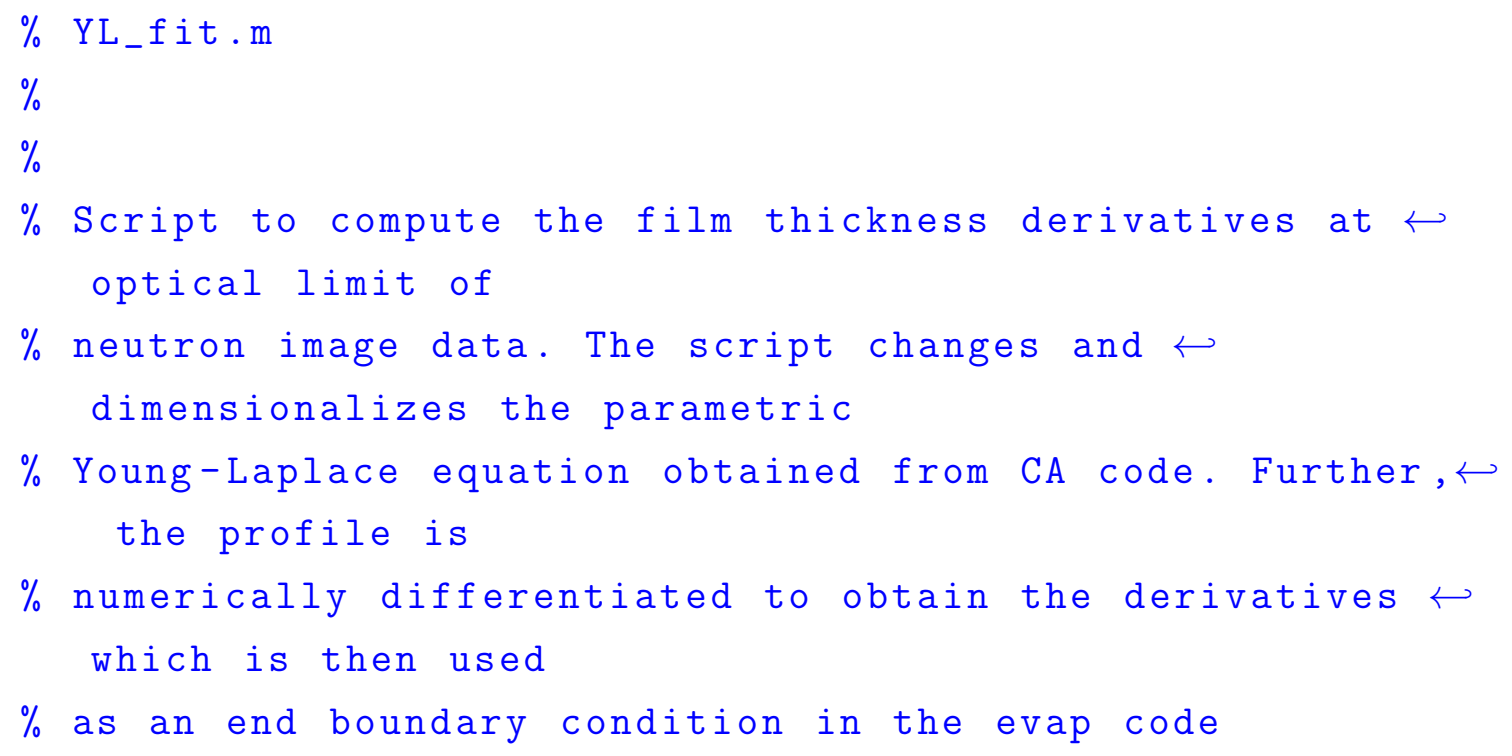


$\%$

\% Written: v1, Kishan Bellur, 06/08/15

\% Modified: Kishan Bellur, 05/05/18

$\%$

$\%$ Ninja comments!

function [y, x, lamda, K1,K2, area,radius] = YL_fit(test_case , fc)

$\%$ load exp data and calcaulate mean temp, rho and sig

split_str=regexp (test_case, ' _ ', 'split');

month=split_str $\{1\}$;

tc=split_str $\{2\}$;

run=split_str $\{3\}$;

$v=\operatorname{split} \_$str $\{4\}$;

sim_path=strjoin( [' . ', split_str(1), strjoin(split_str $\left.(1: 2),{ }^{\prime}-1\right), \ldots$

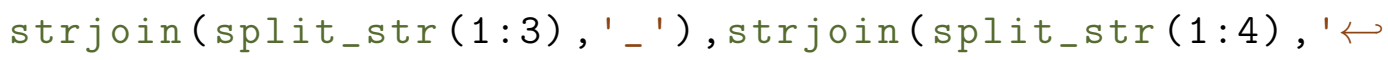
$-1)], 1 / 1)$;

exp_path=sim_path;

if $\operatorname{length}(v)==2$

exp_path $($ end $-1: \operatorname{end}+1)=\exp ^{\prime}$;

else

exp_path $($ end $-2:$ end $)={ }^{\prime} \exp { }^{\prime} ;$

end

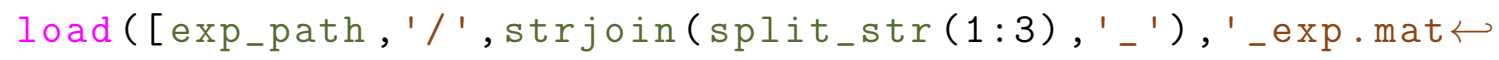
1]) 


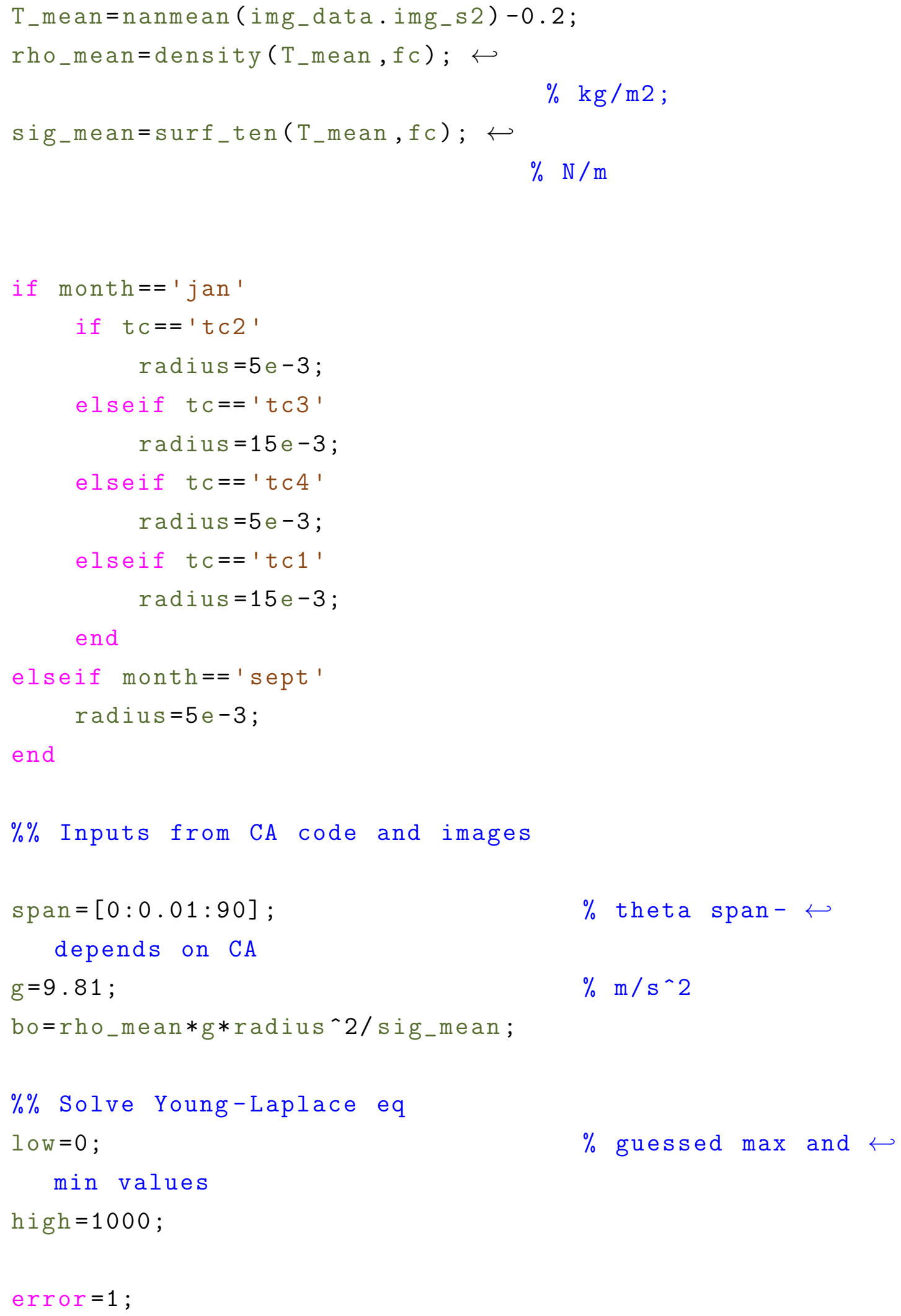




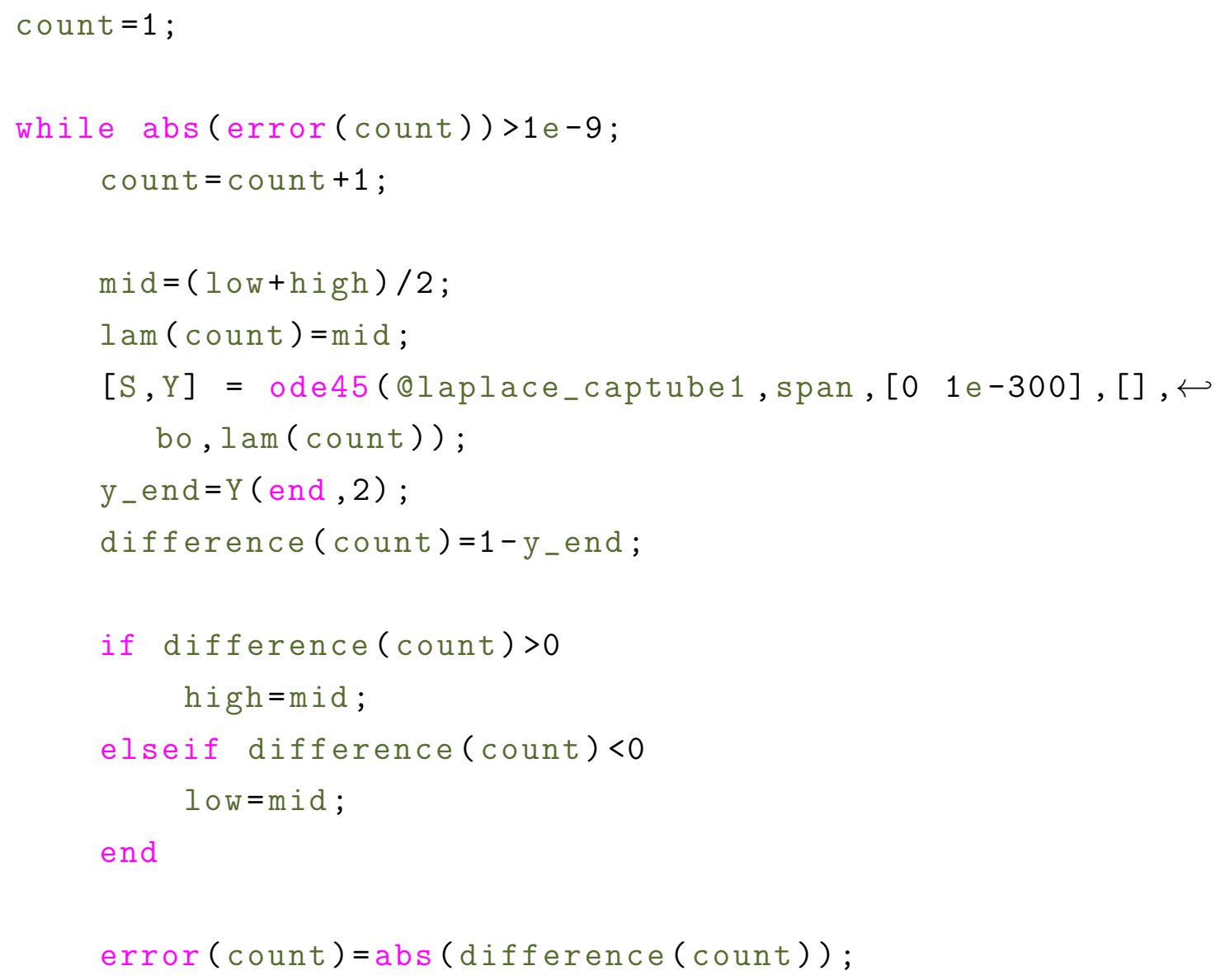




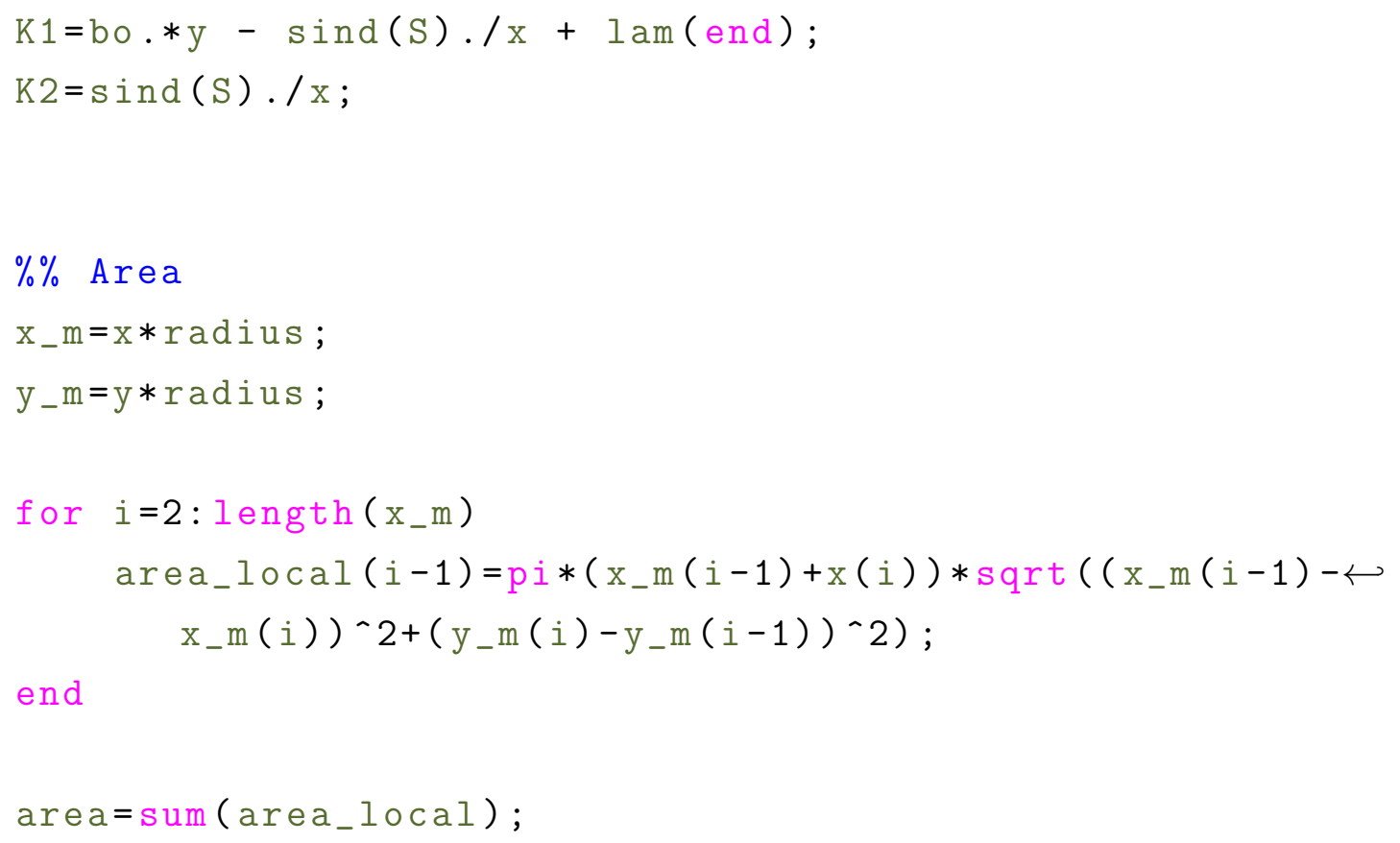

\section{B.2 alpha_finder.m}

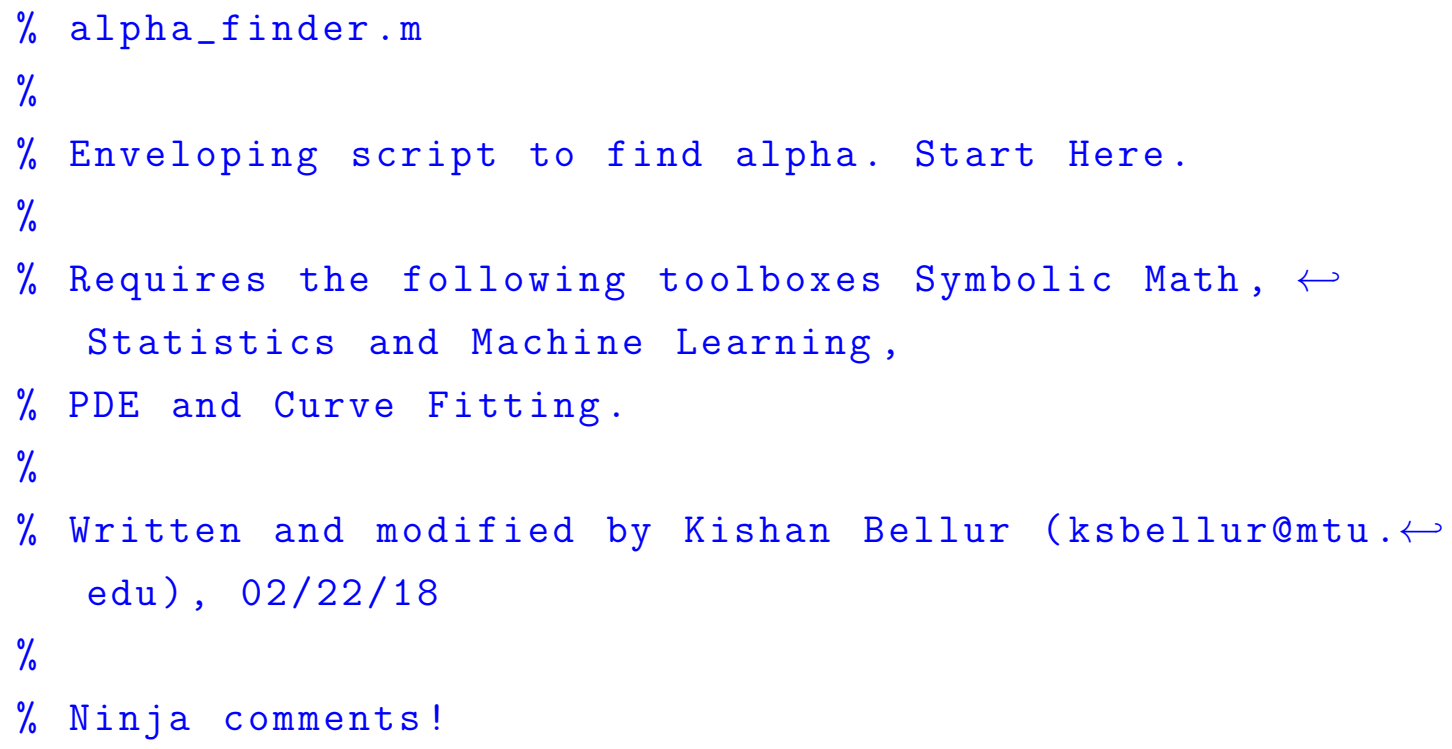




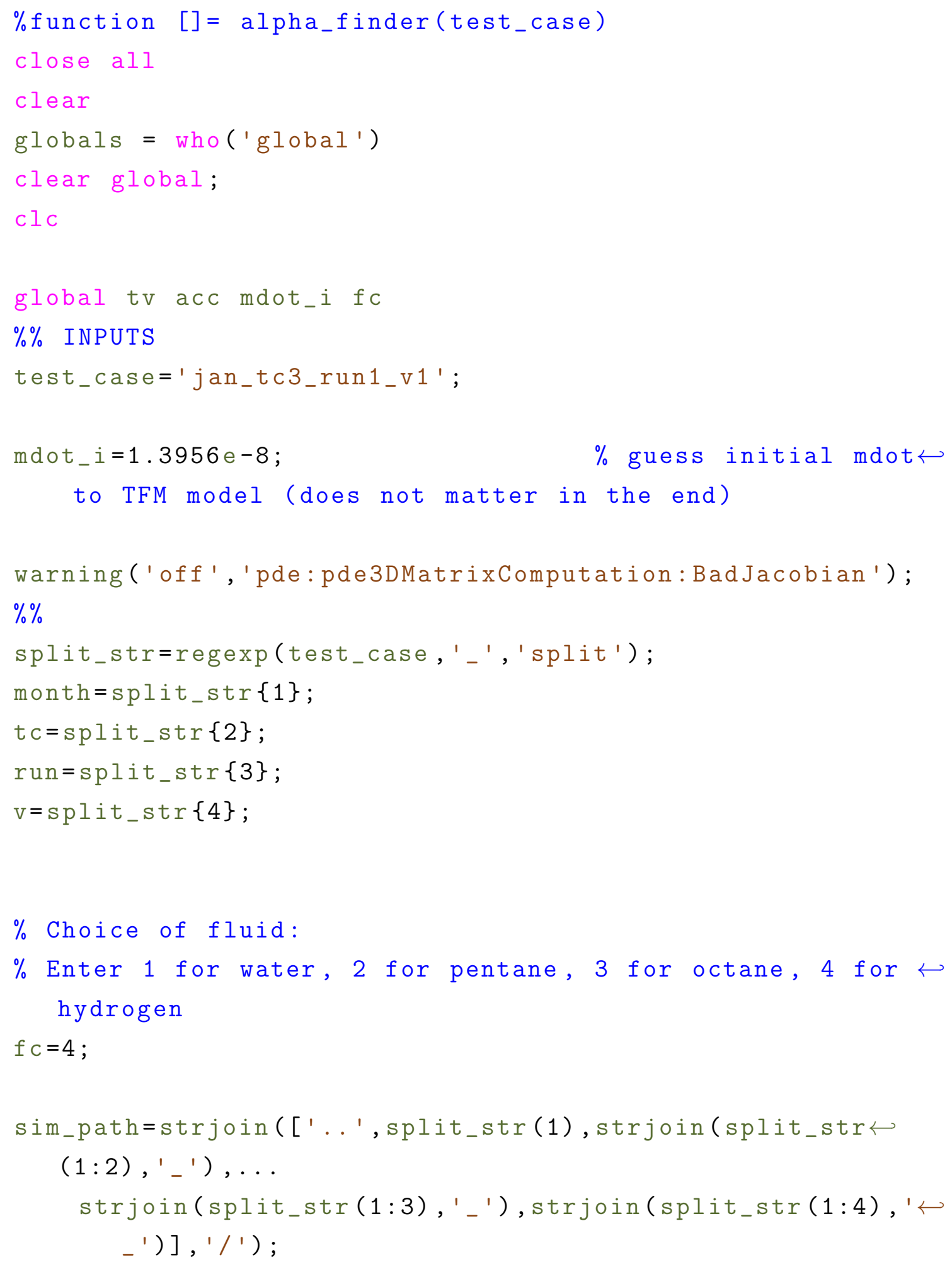




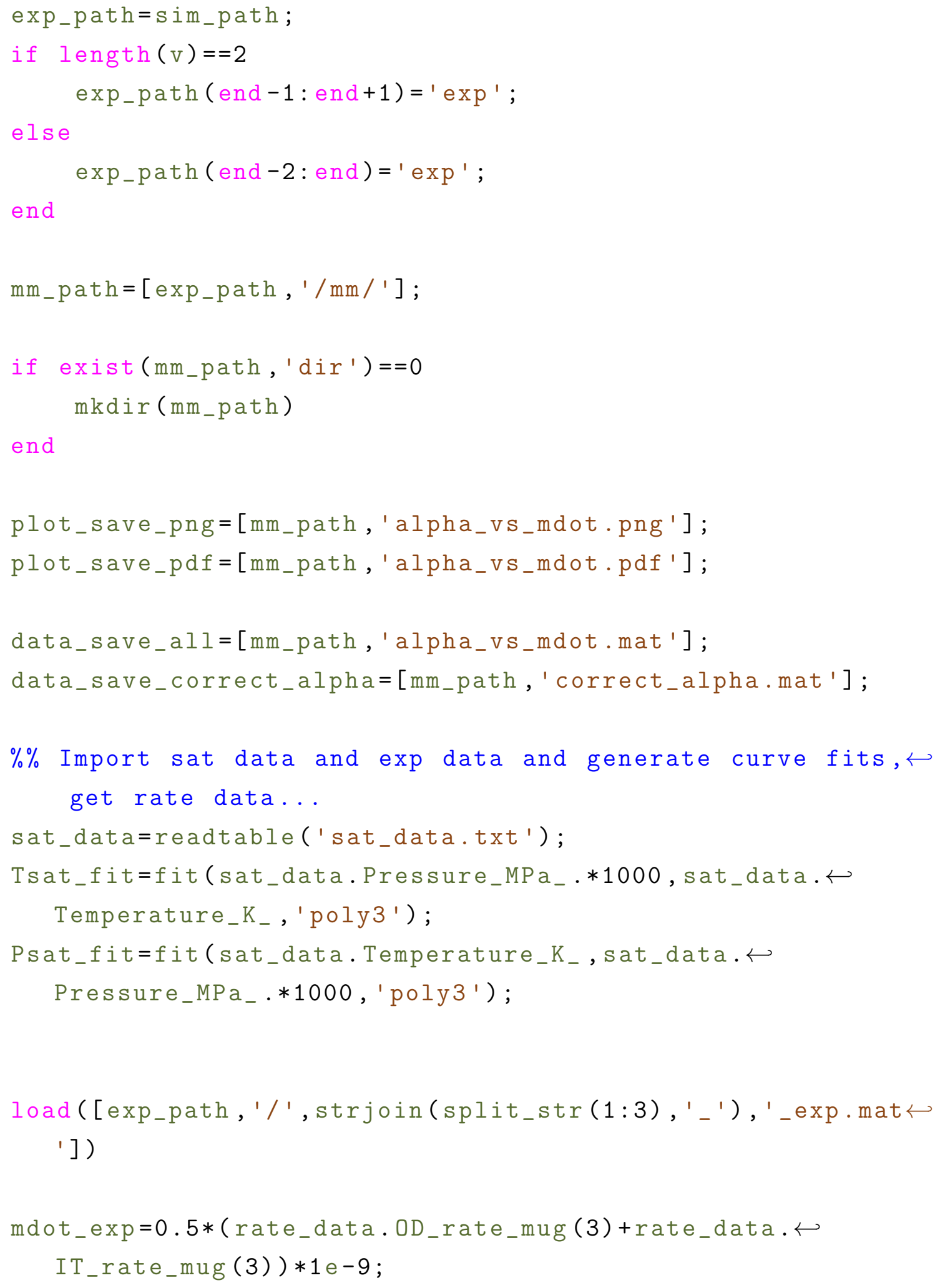




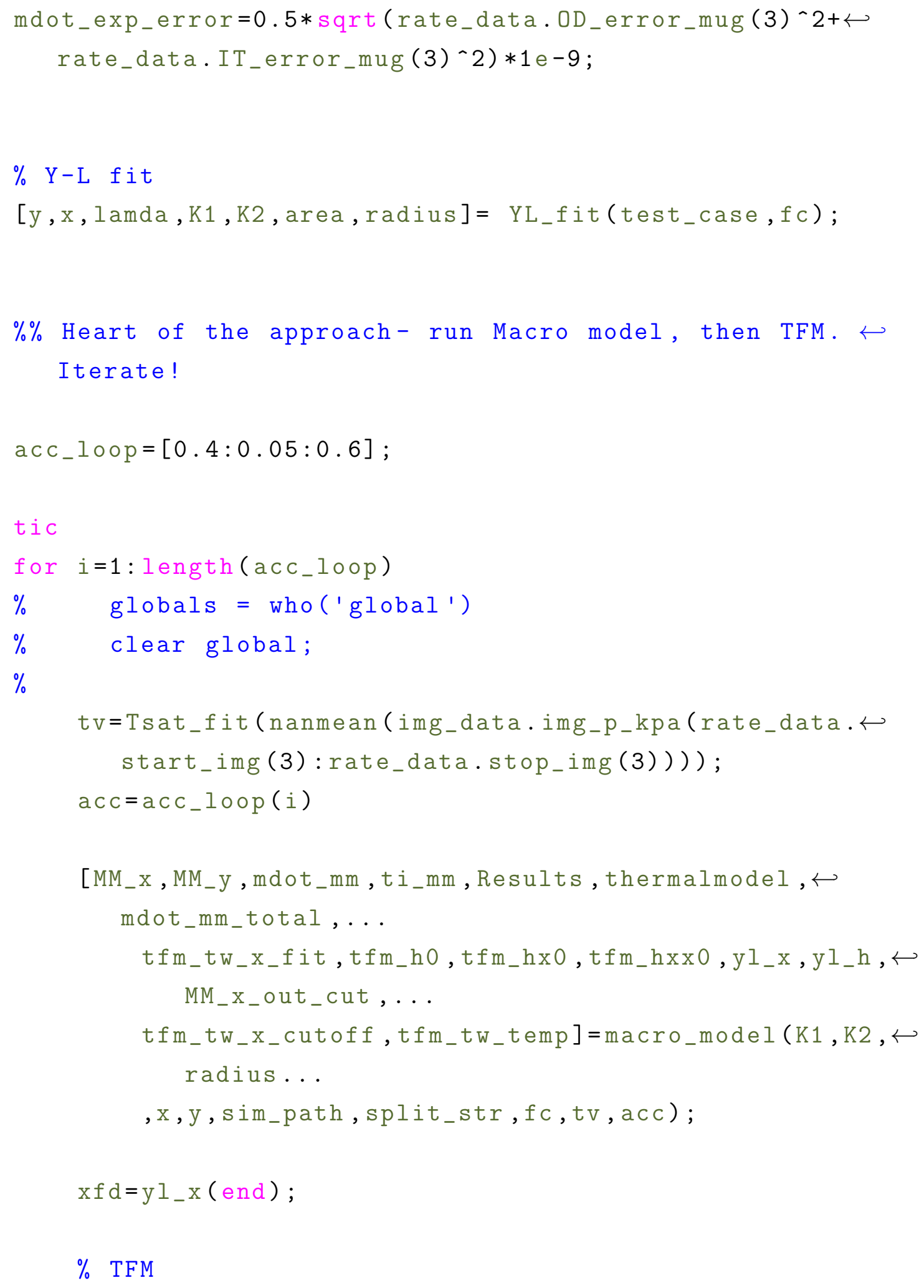




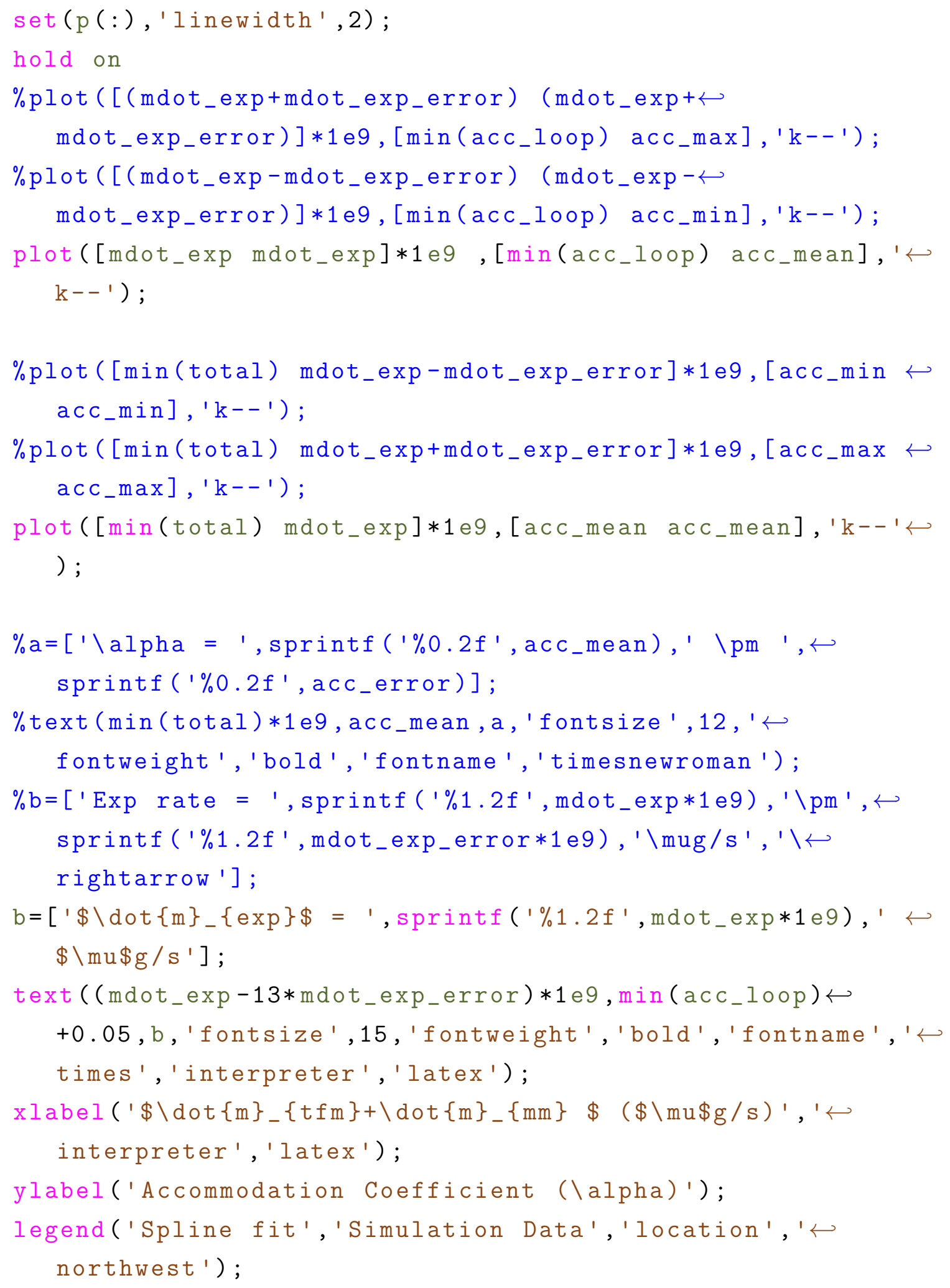




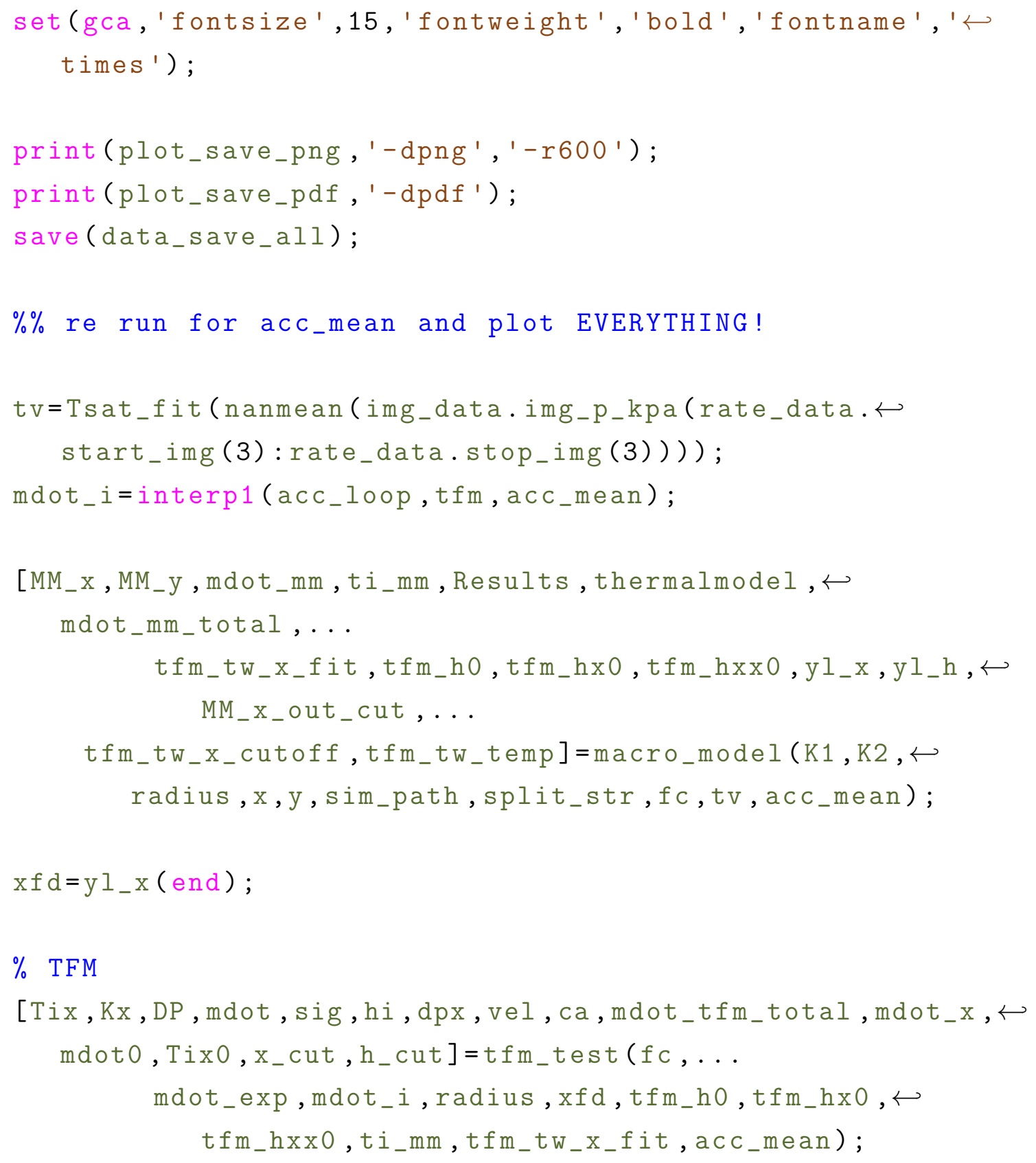




\section{B.3 const_ac_mdot.m}

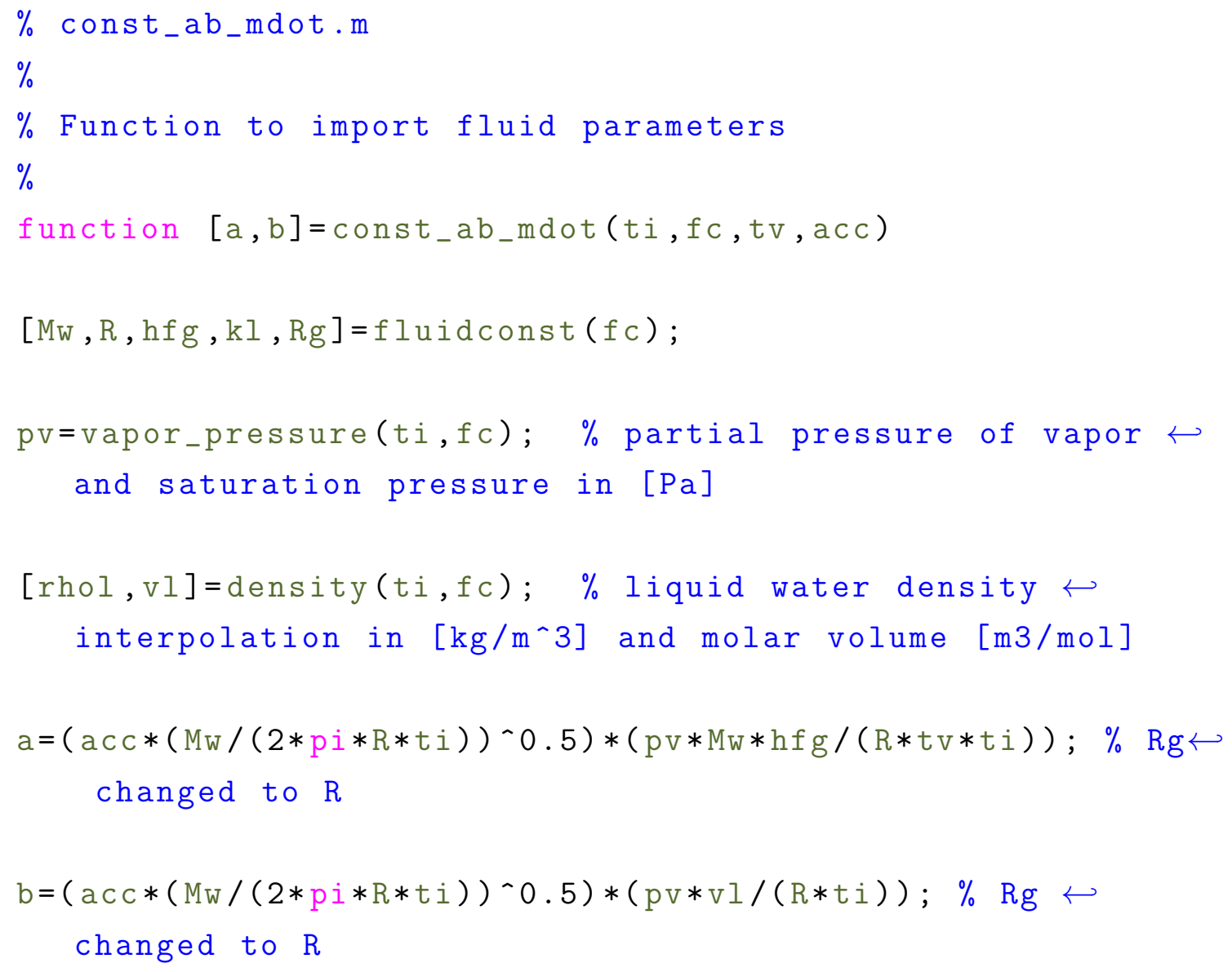

\section{B.4 curvaturek.m}

$\%$ curvaturek.m

$\%$ 


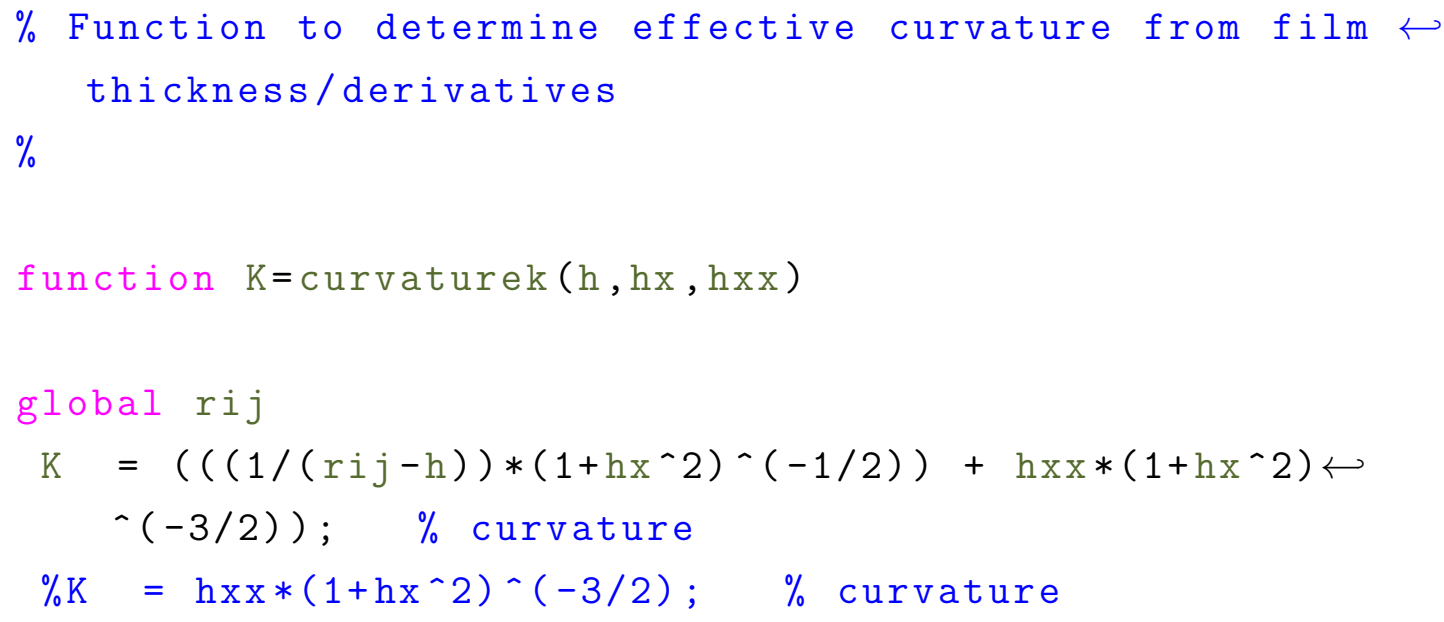

\section{B.5 density.m}

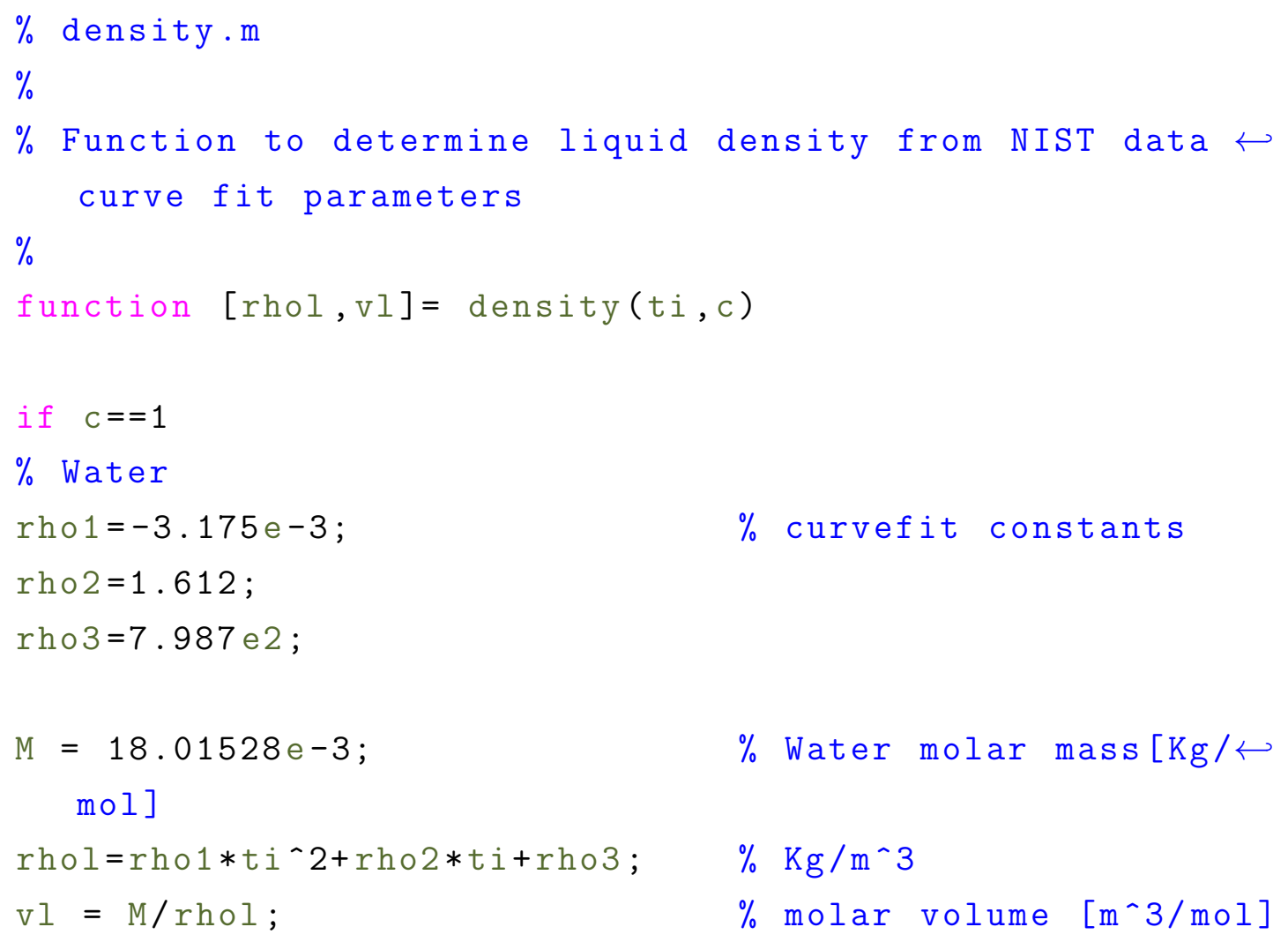




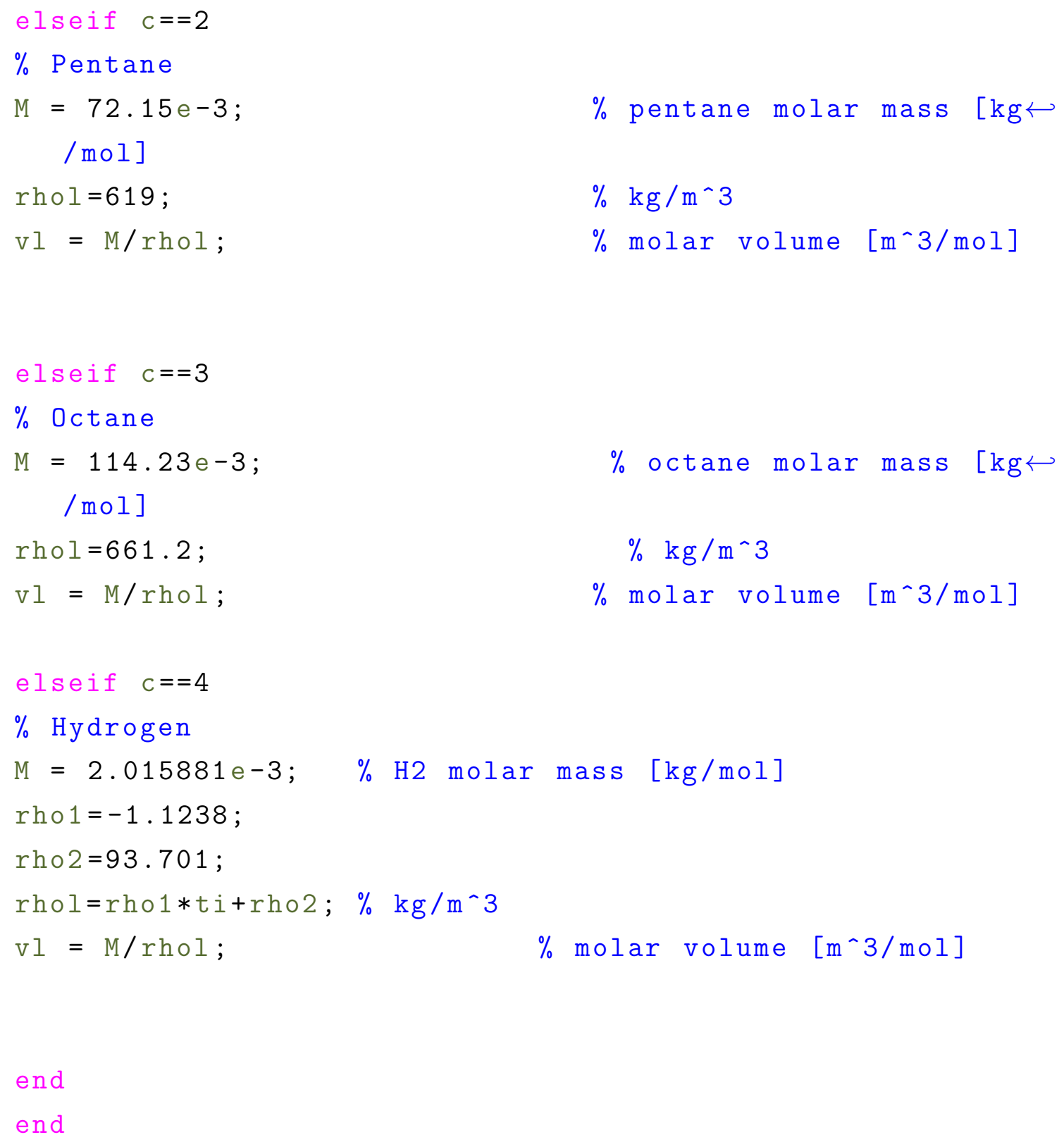

\section{B.6 diff_eq.m}




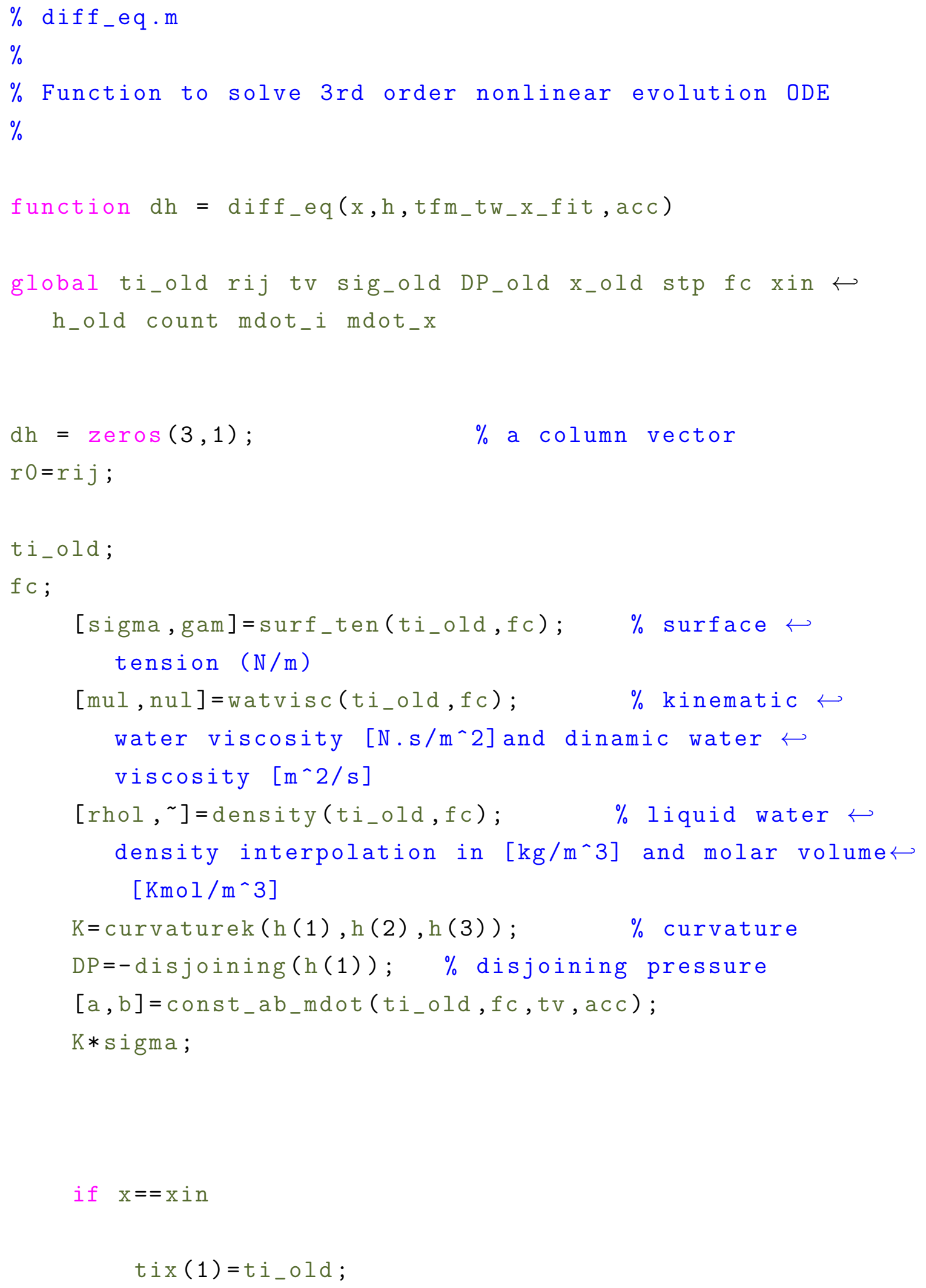




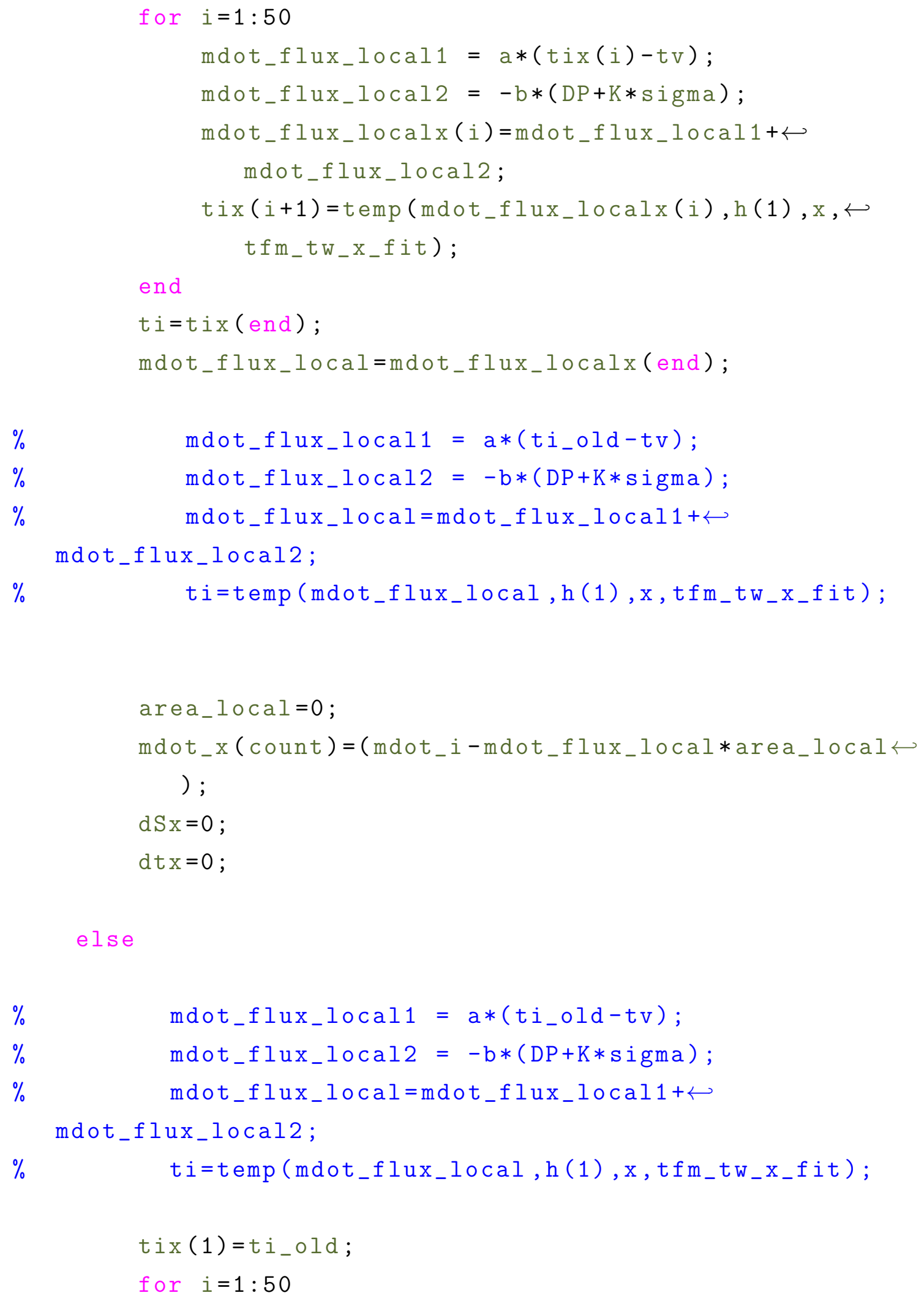




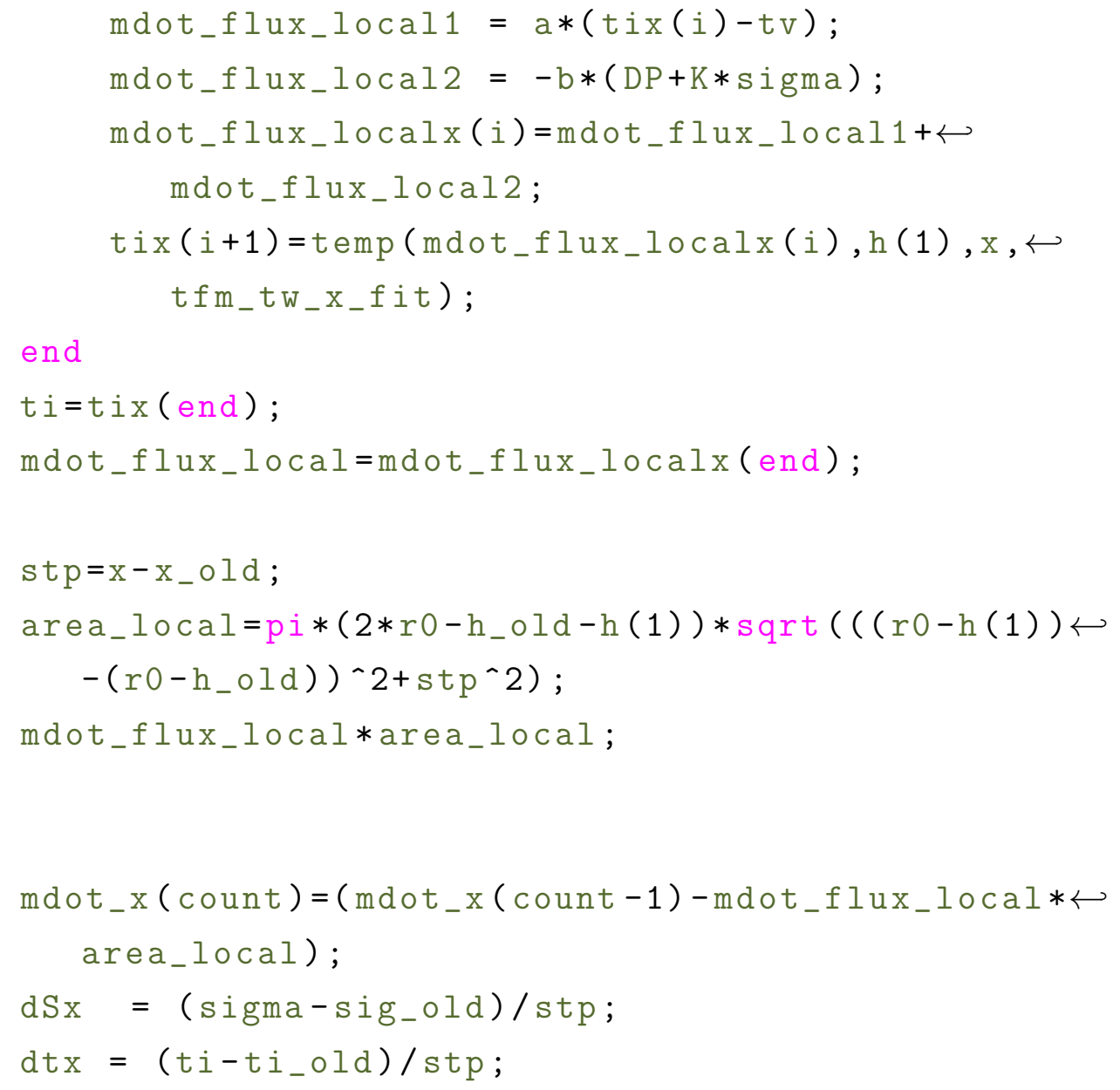




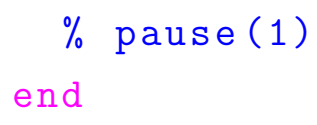

\section{B.7 disjoining.m}

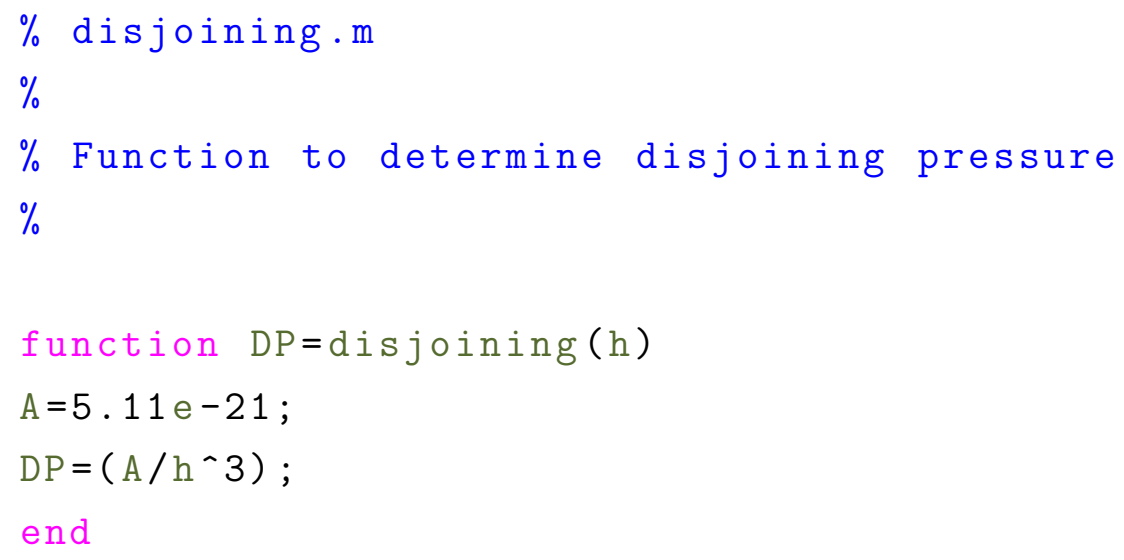

\section{B.8 externalHeatFlux.m}

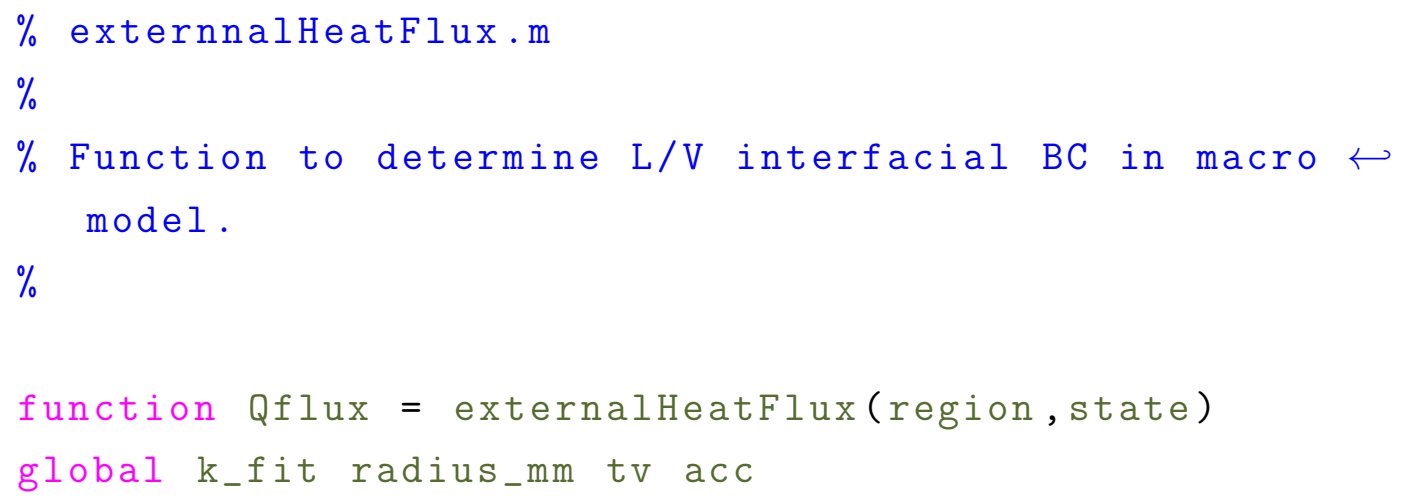




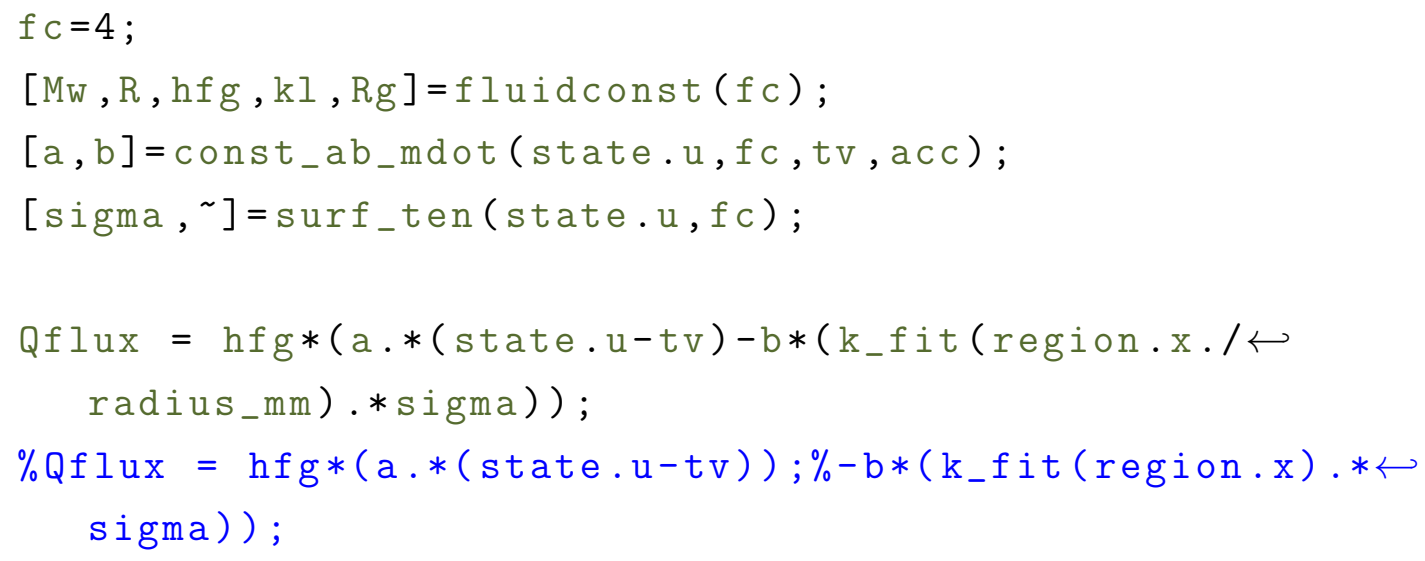

\section{B.9 fluidconst.m}

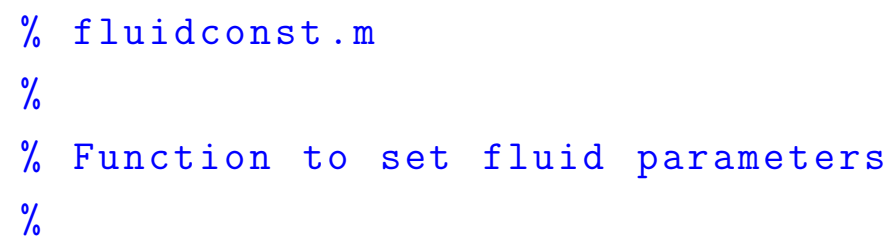




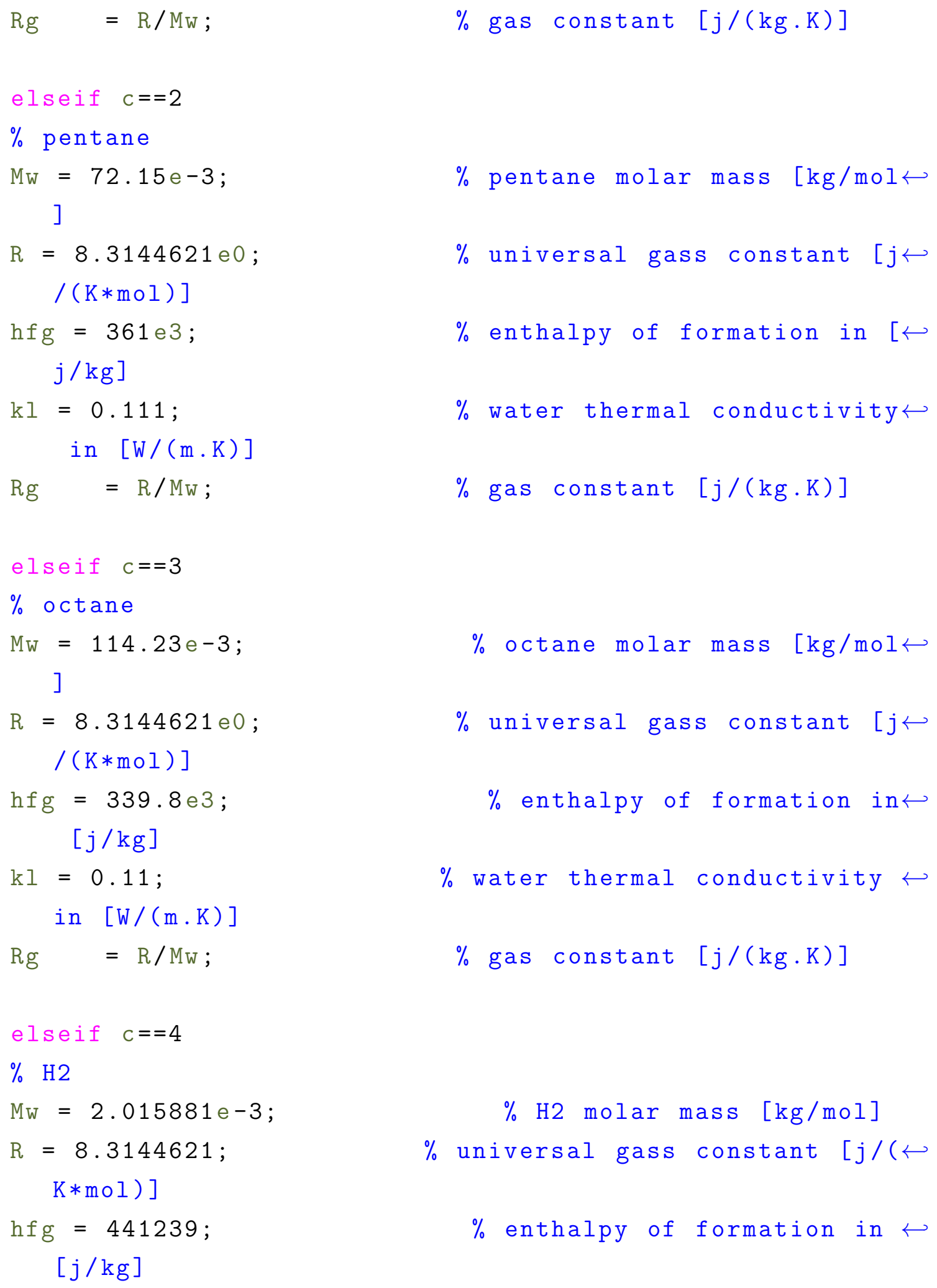



$\mathrm{kl}=0.103$
$\%$ thermal conductivity in $[\mathrm{W} \hookleftarrow$ $/(\mathrm{m} \cdot \mathrm{K})]$
$\operatorname{Rg} \quad=R / M_{w}$;
$\%$ gas constant $[j /(\mathrm{kg} \cdot \mathrm{K})]$

end

end

\section{B.10 kTix.m}

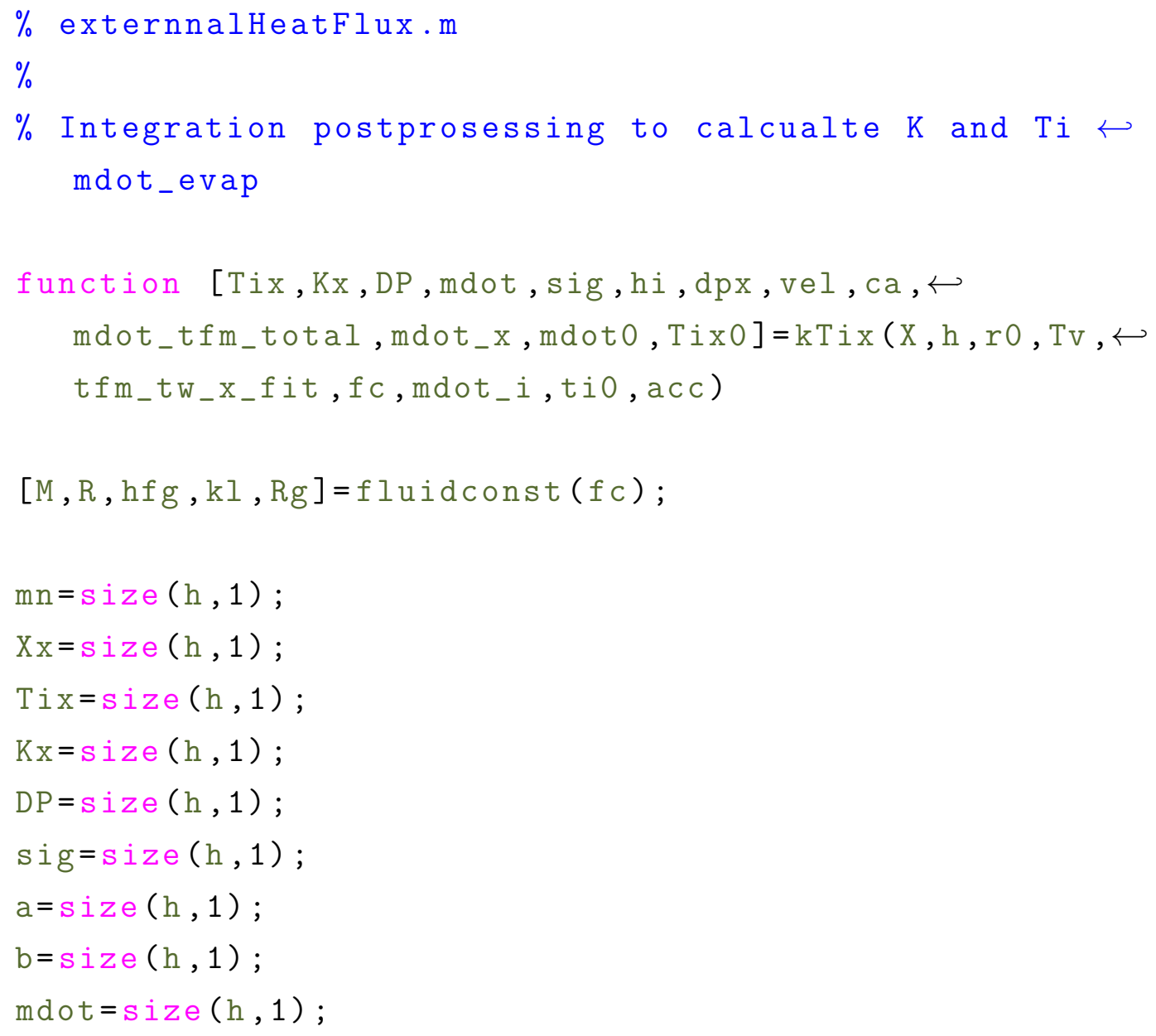




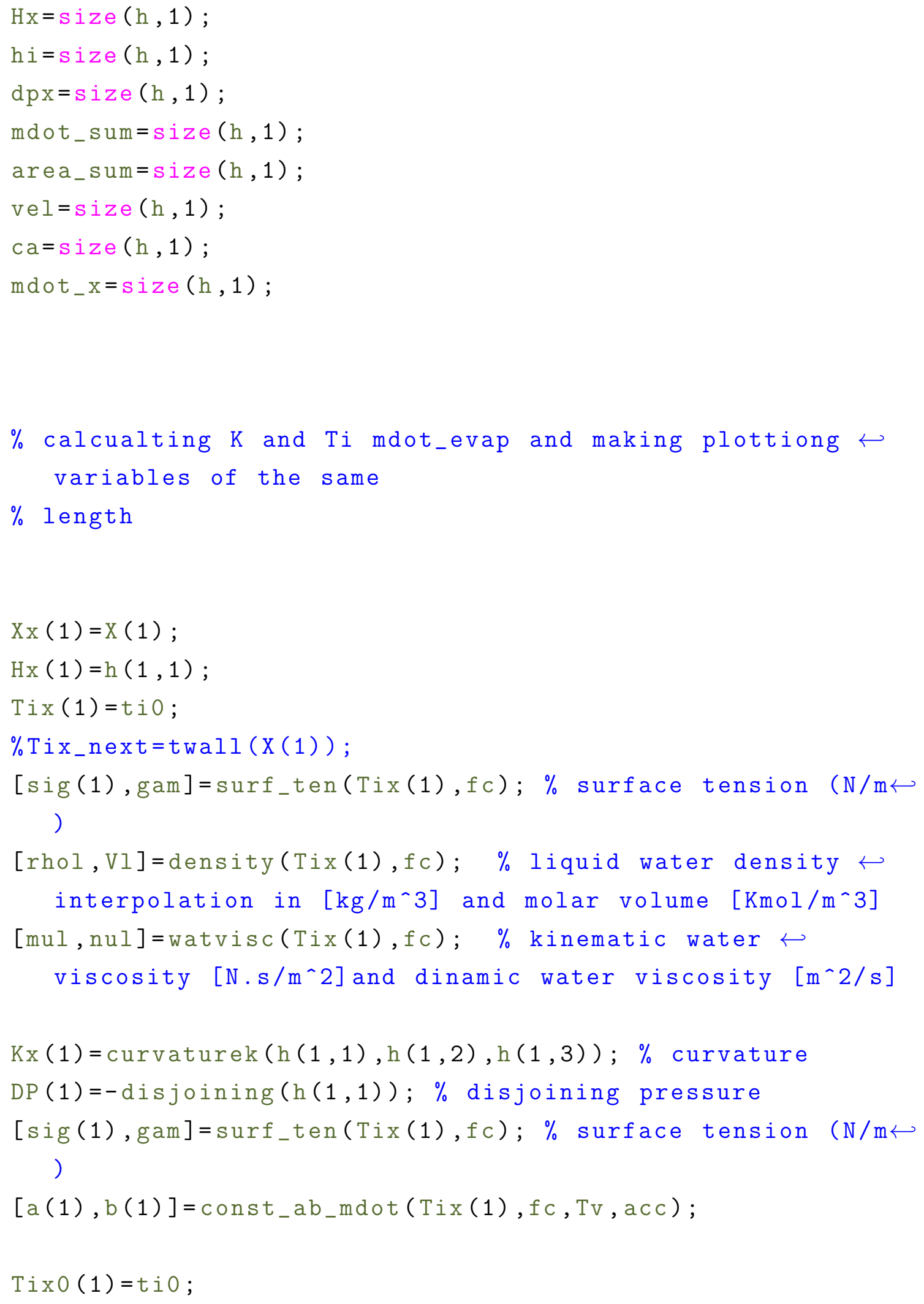

$\%$ calcualting $K$ and $\mathrm{Ti}$ mdot_evap and making plottiong $\hookleftarrow$ variables of the same

$\%$ length 


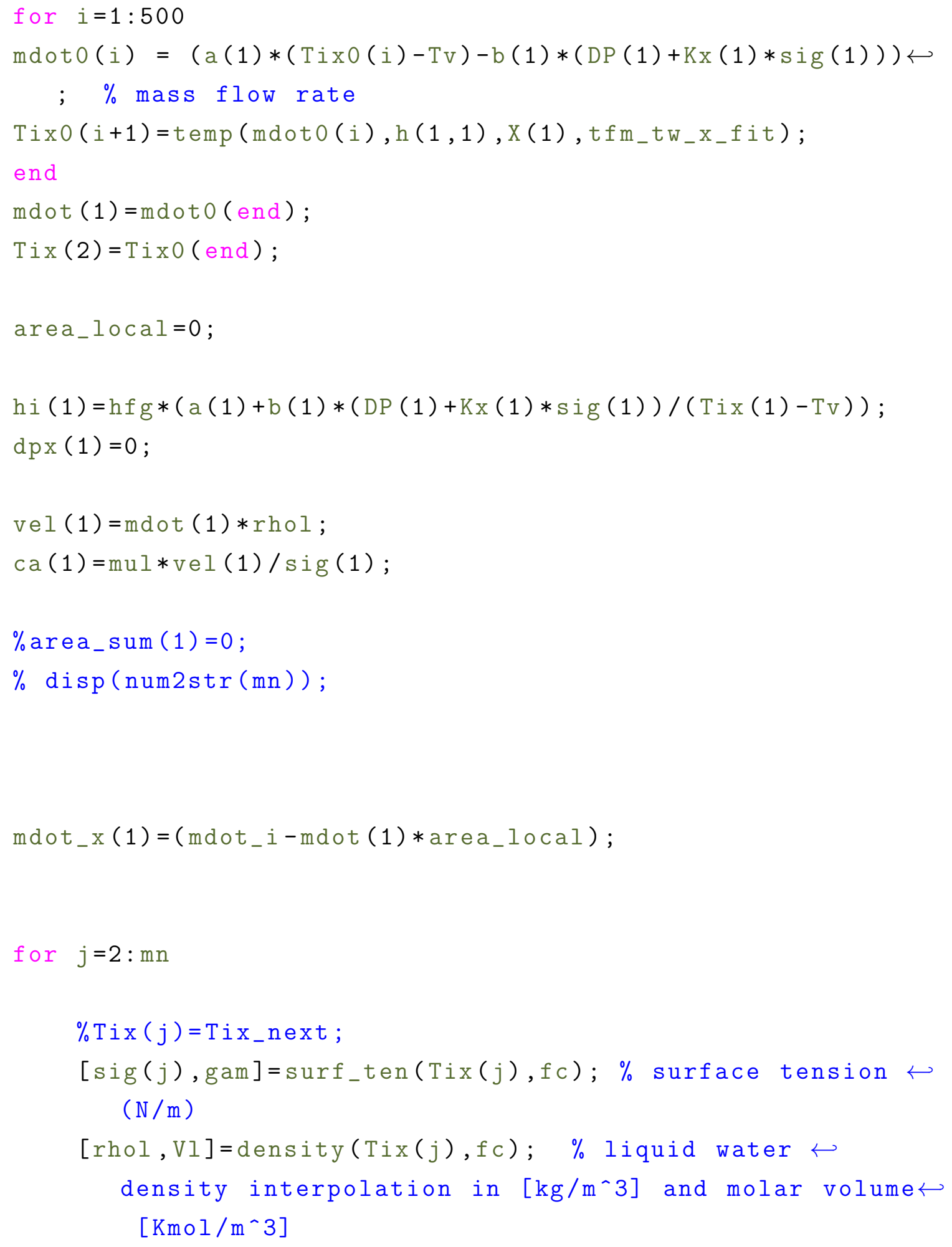




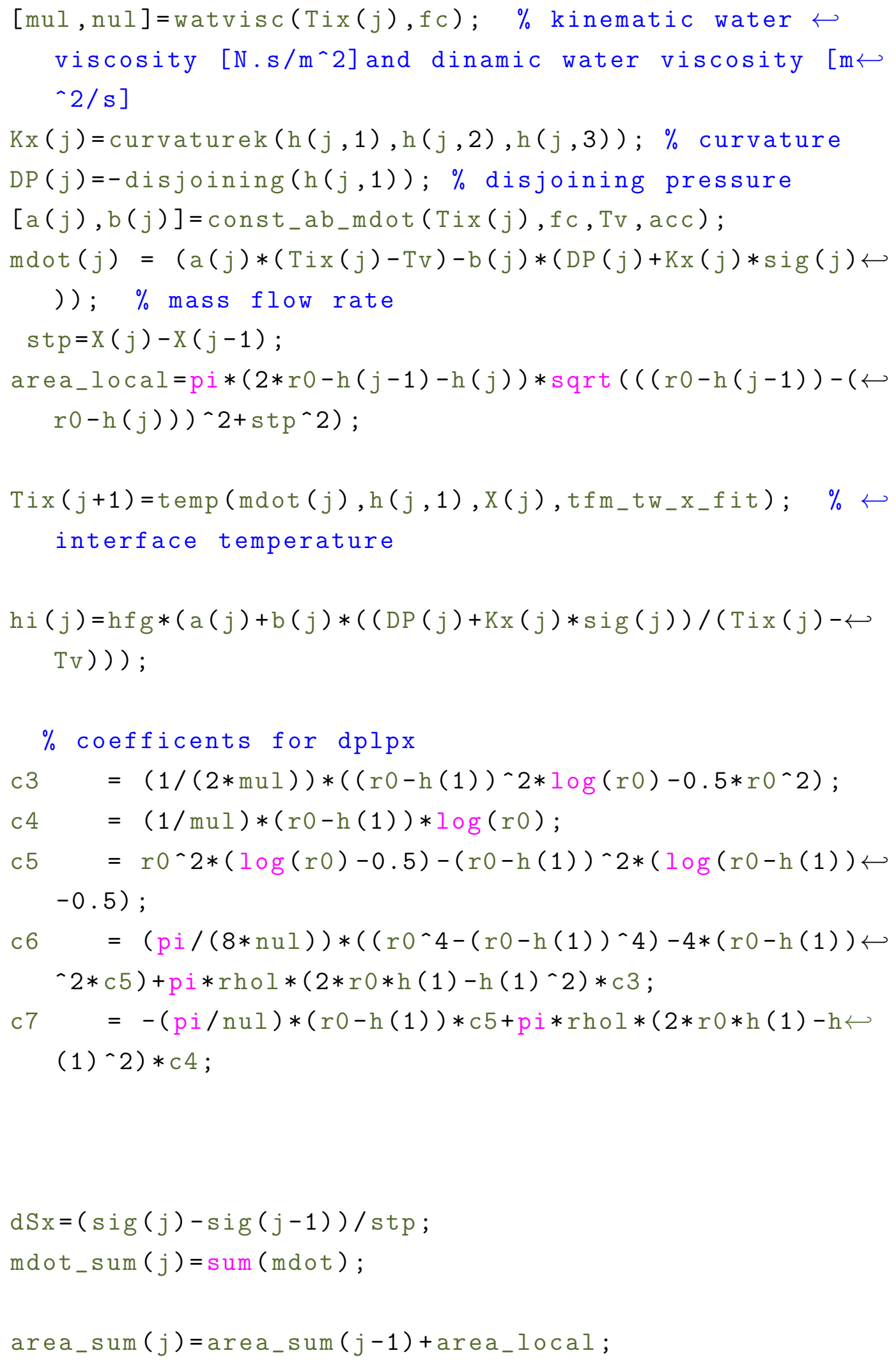




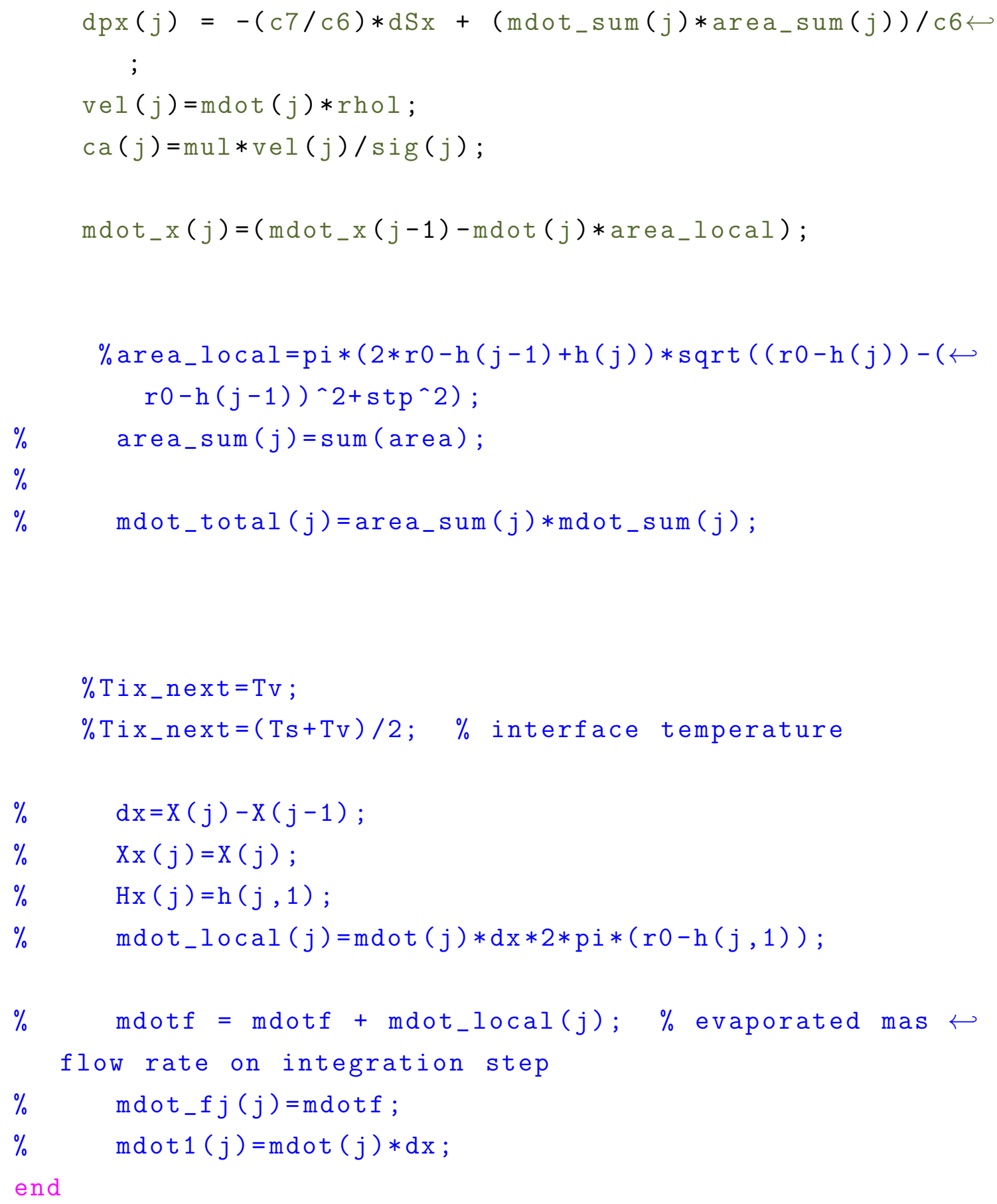




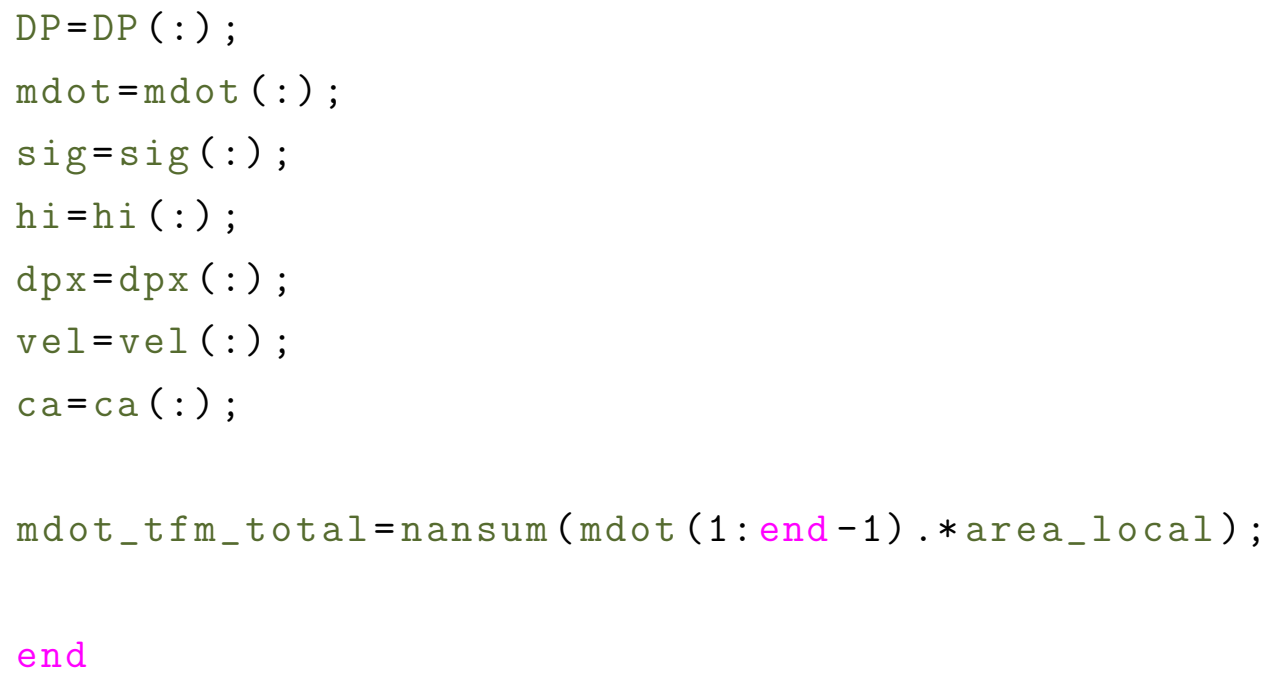

\section{B.11 kTix.m}

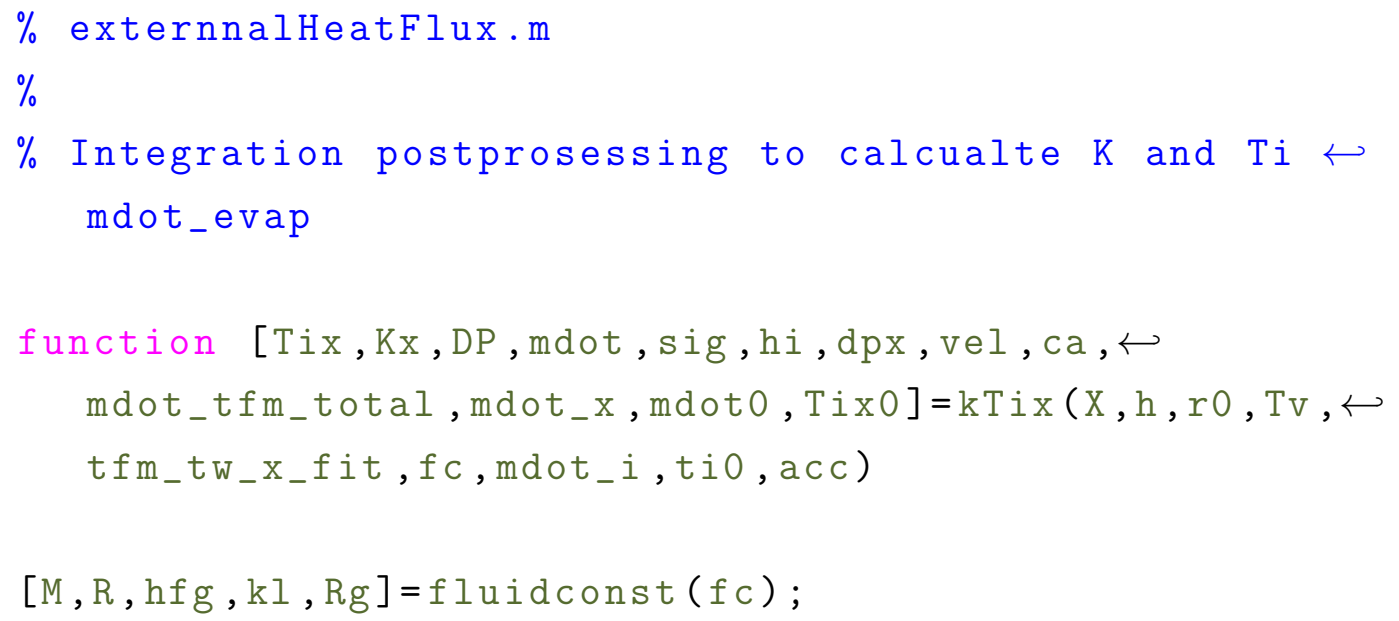




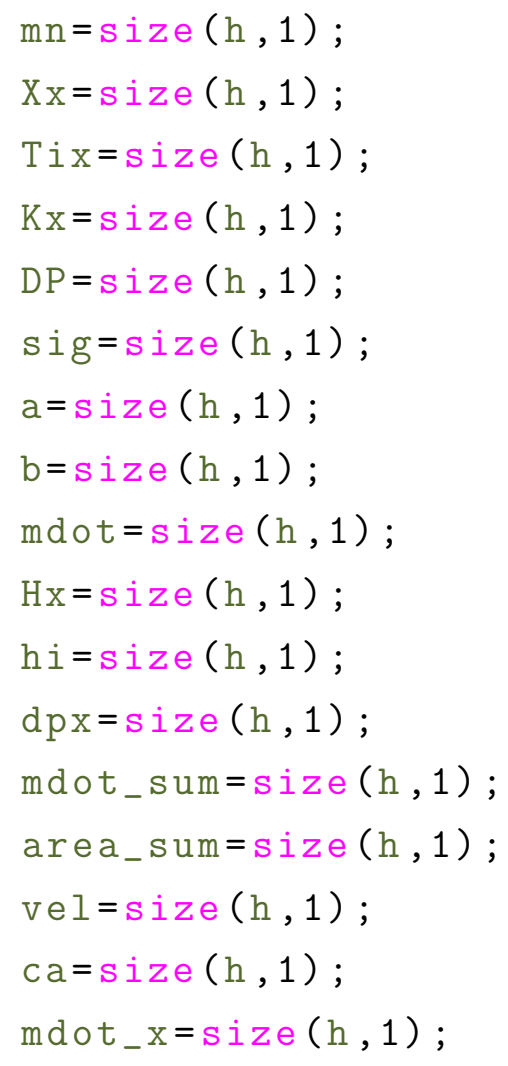

$\%$ calcualting $K$ and $\mathrm{Ti}$ mdot_evap and making plottiong $\hookleftarrow$ variables of the same

$\%$ length

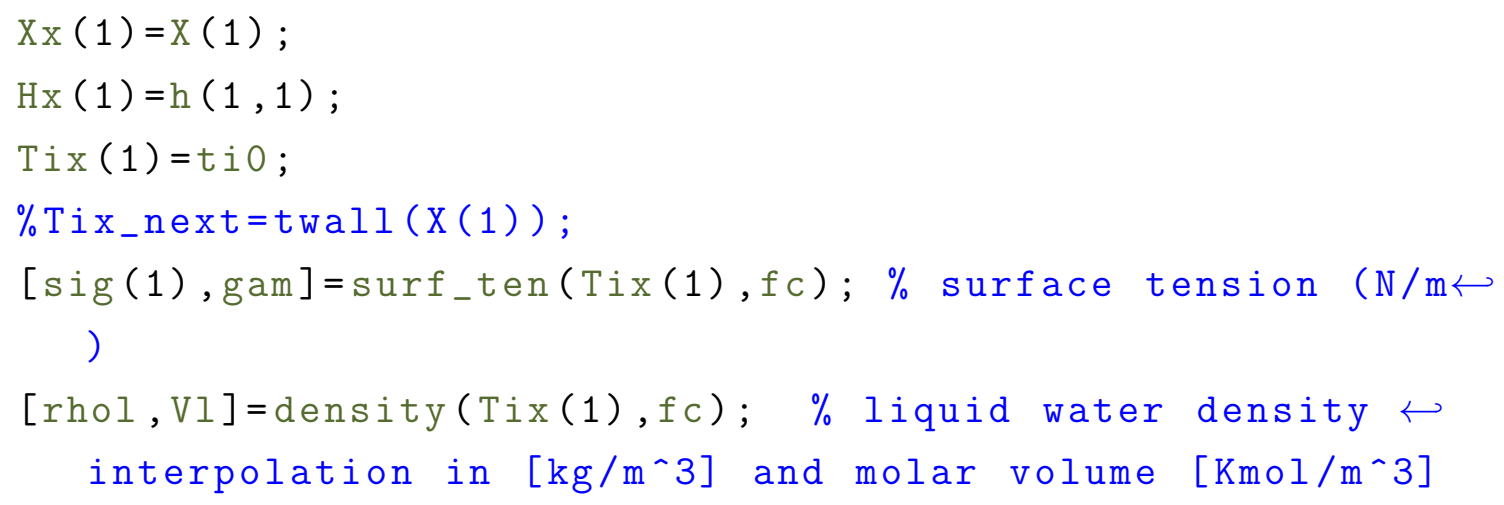




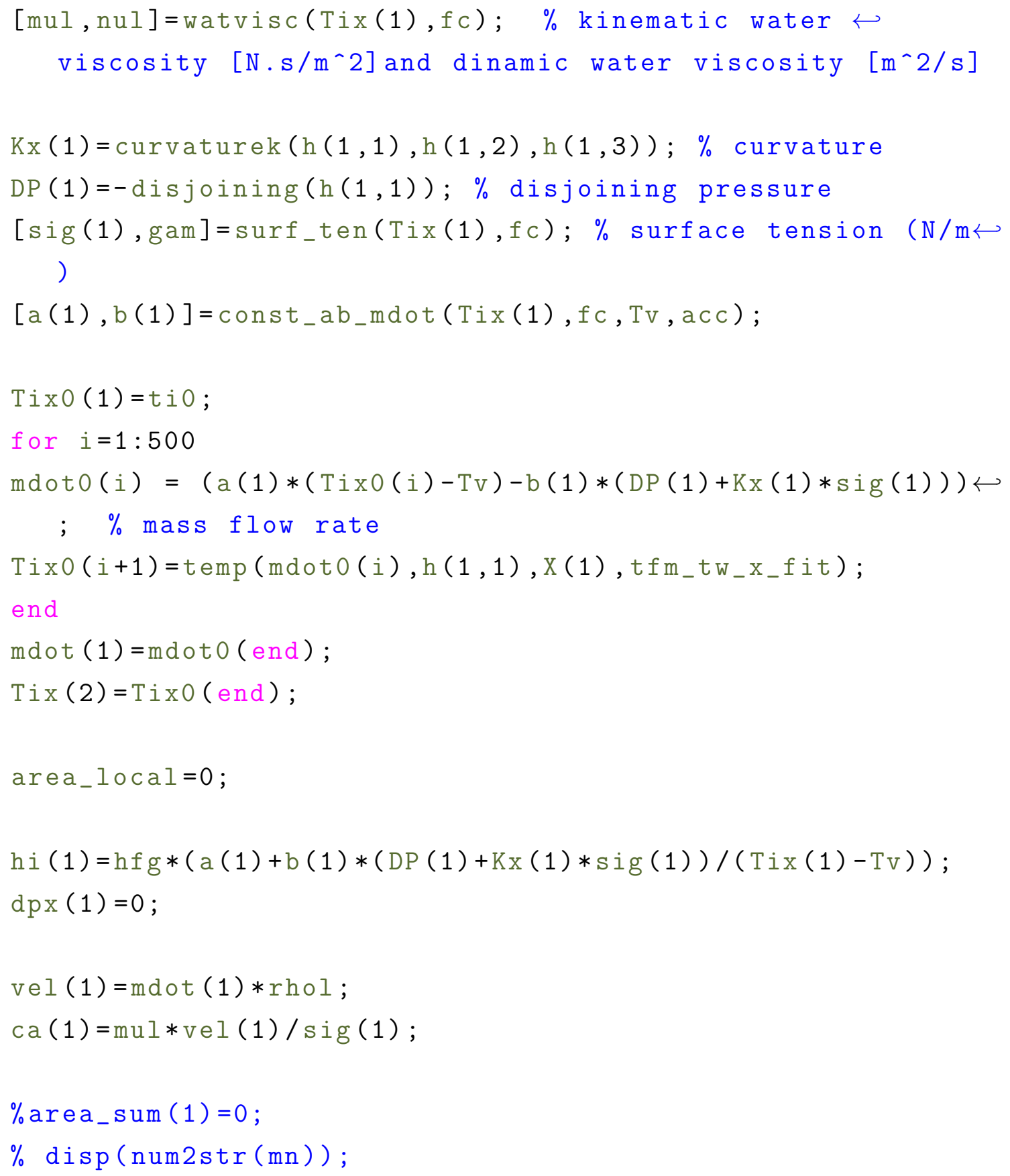


for $j=2: m n$

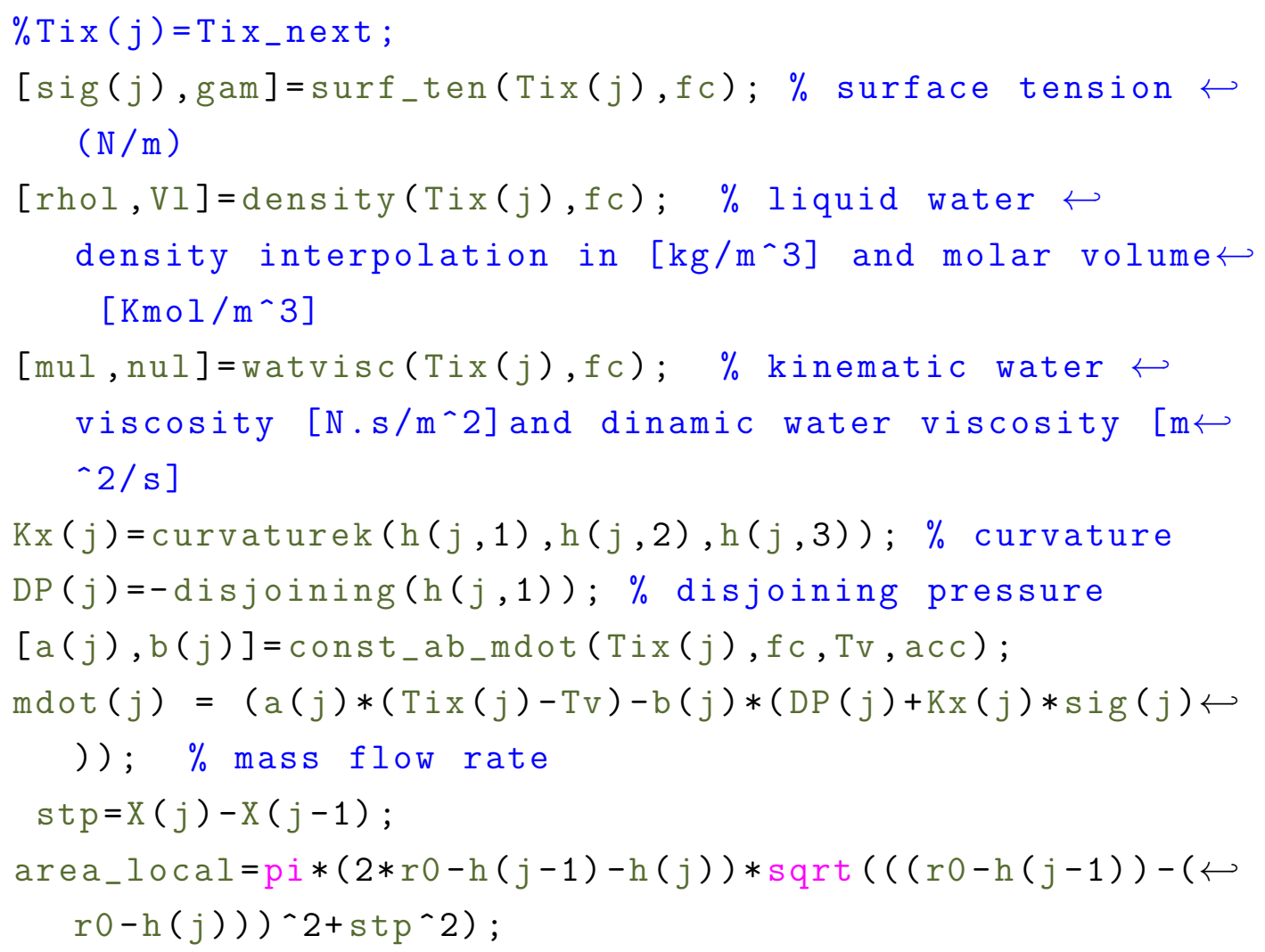



$\left.(1)^{\wedge} 2\right) * c 4$;

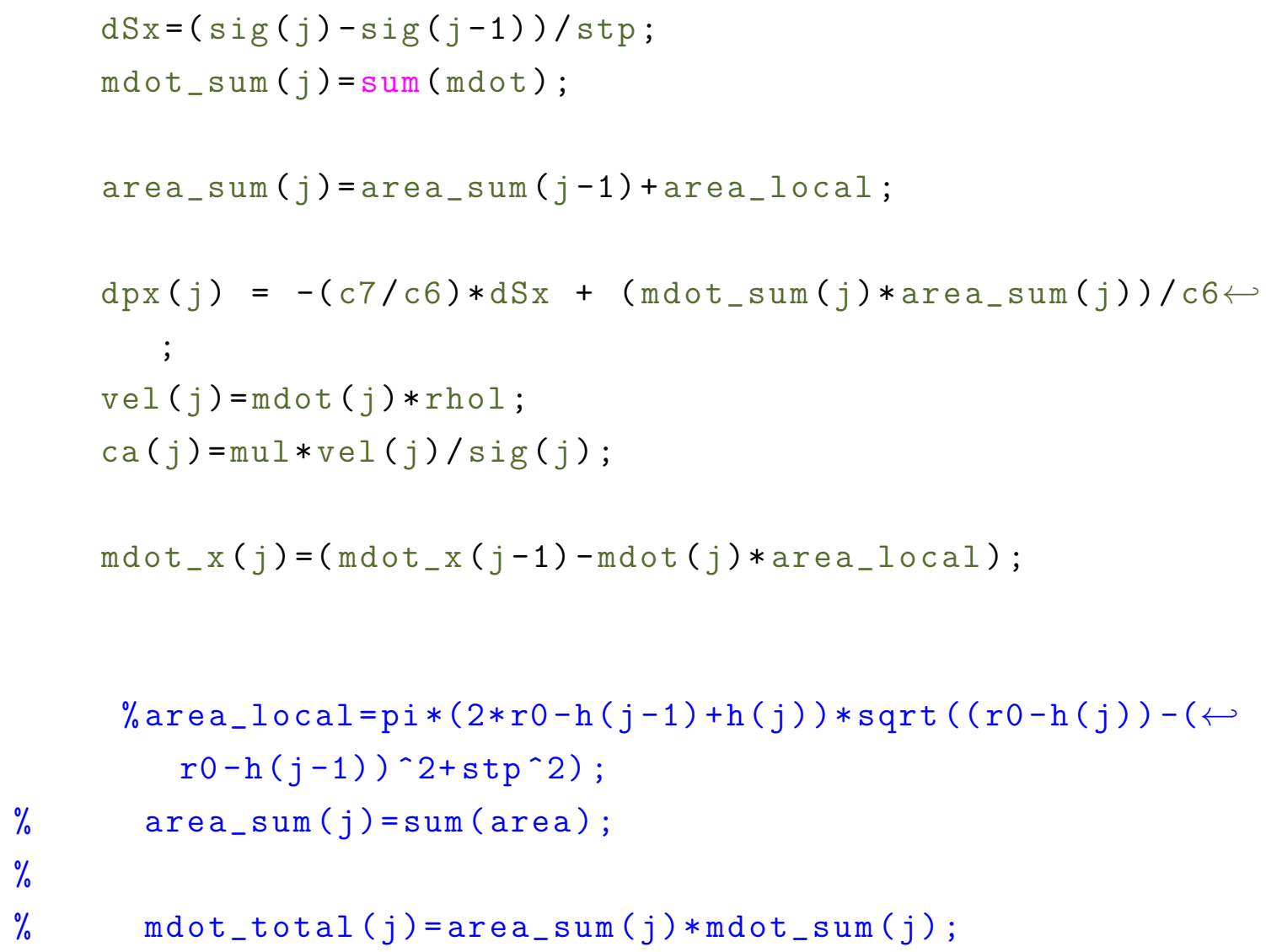




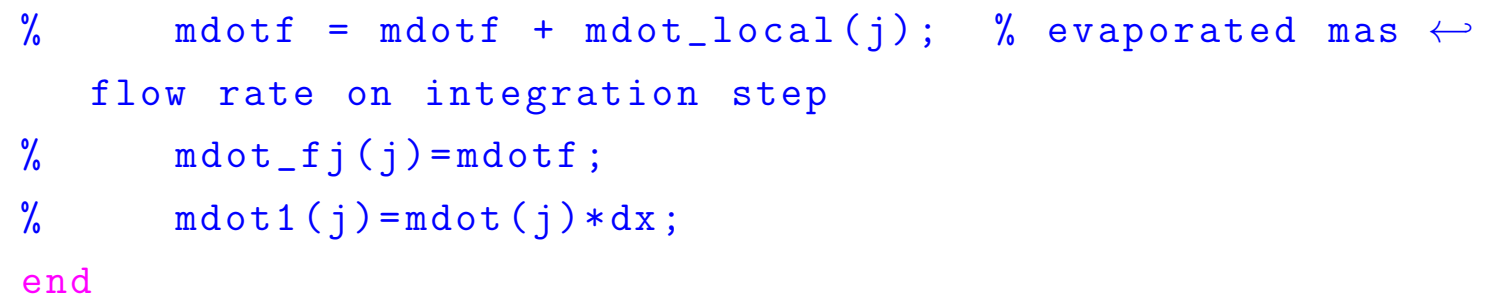

$\% \operatorname{mdot}($ end ) $* 1.895942730357007 e-04$

$\%$ mdot_evap $=$ mdot_total (end) + mdot (end) $\hookleftarrow$ *1.895942730357007e-04;

\section{B.12 macro_model.m}

\% macro_model_test.m $\%$ 


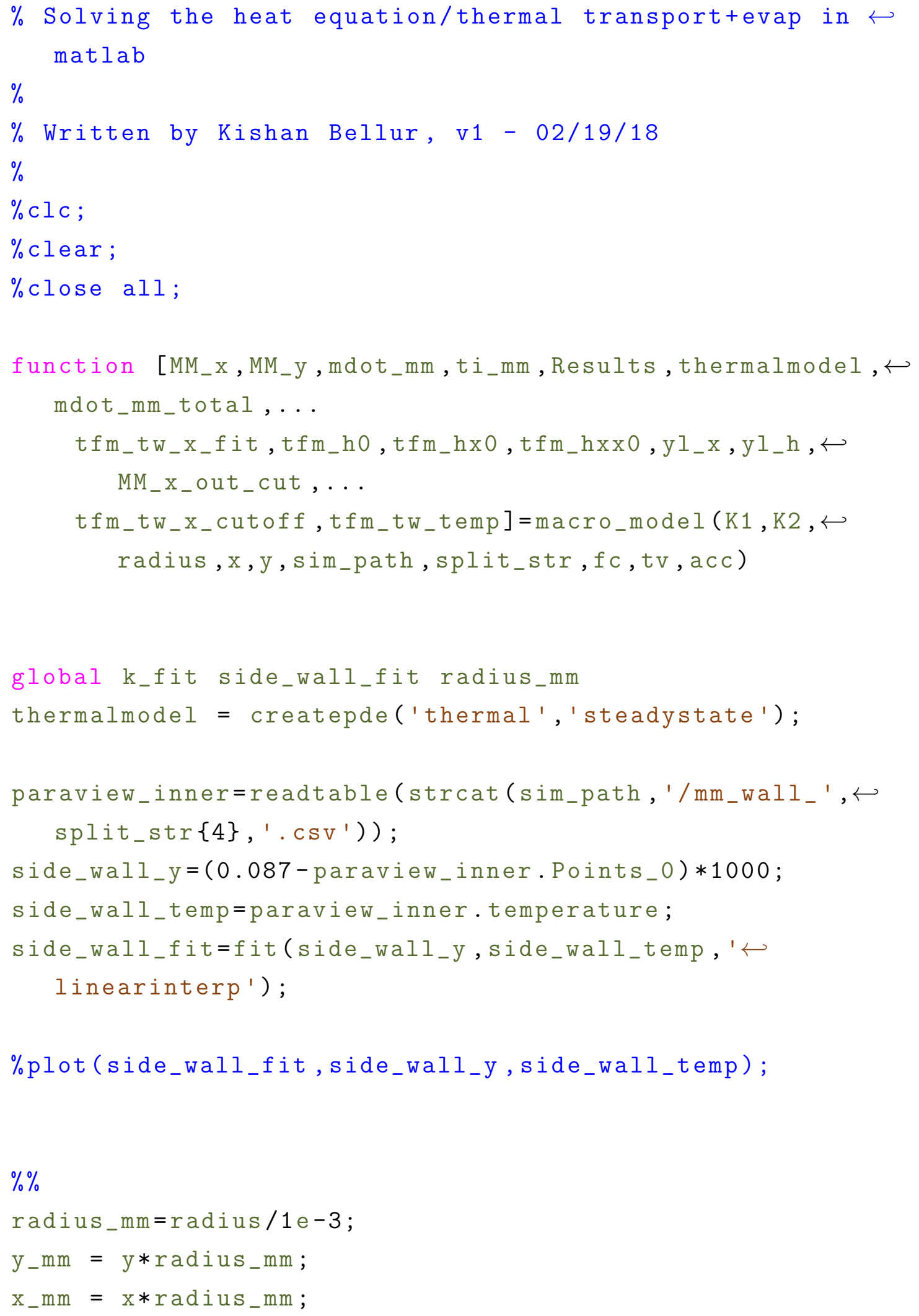




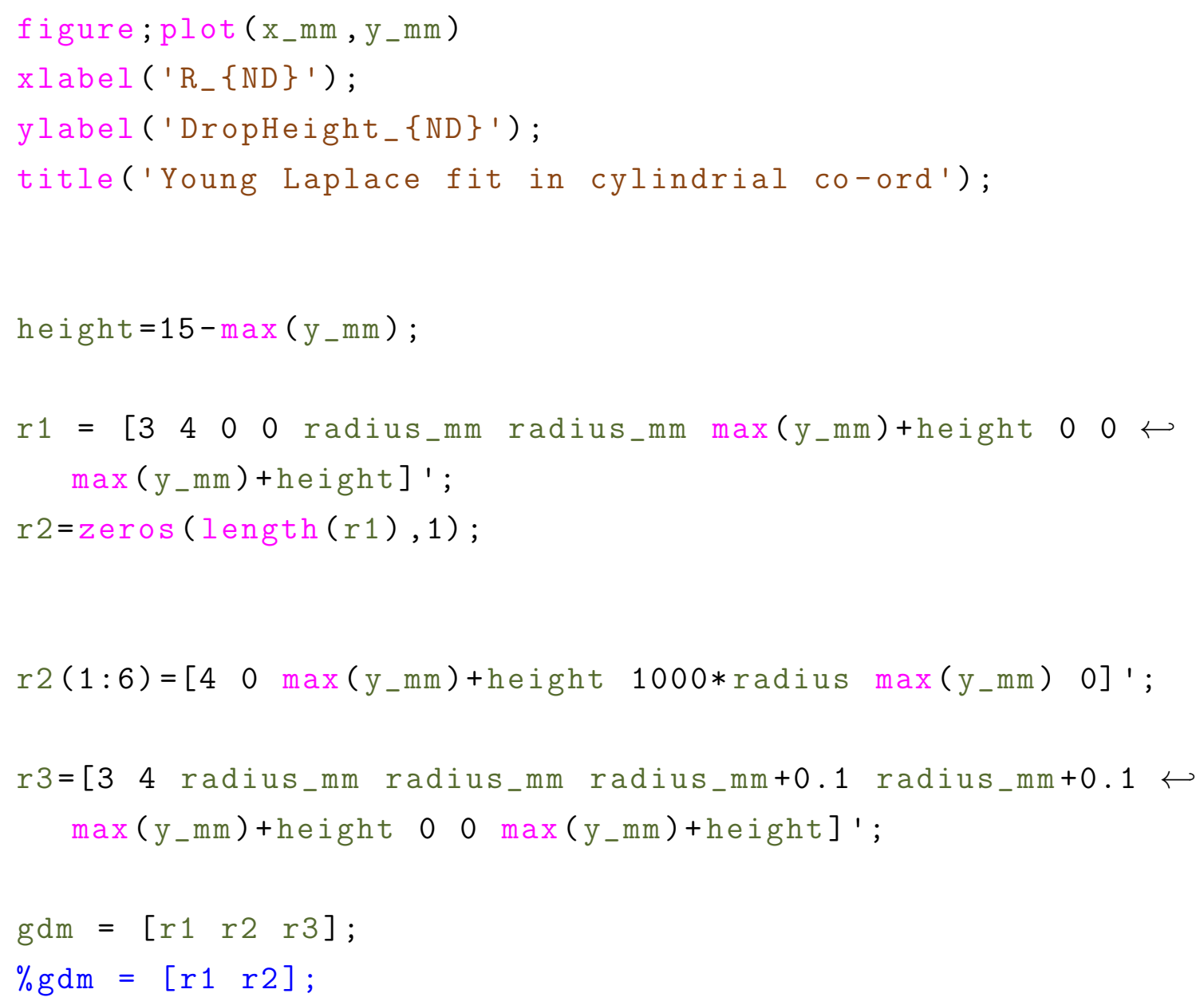




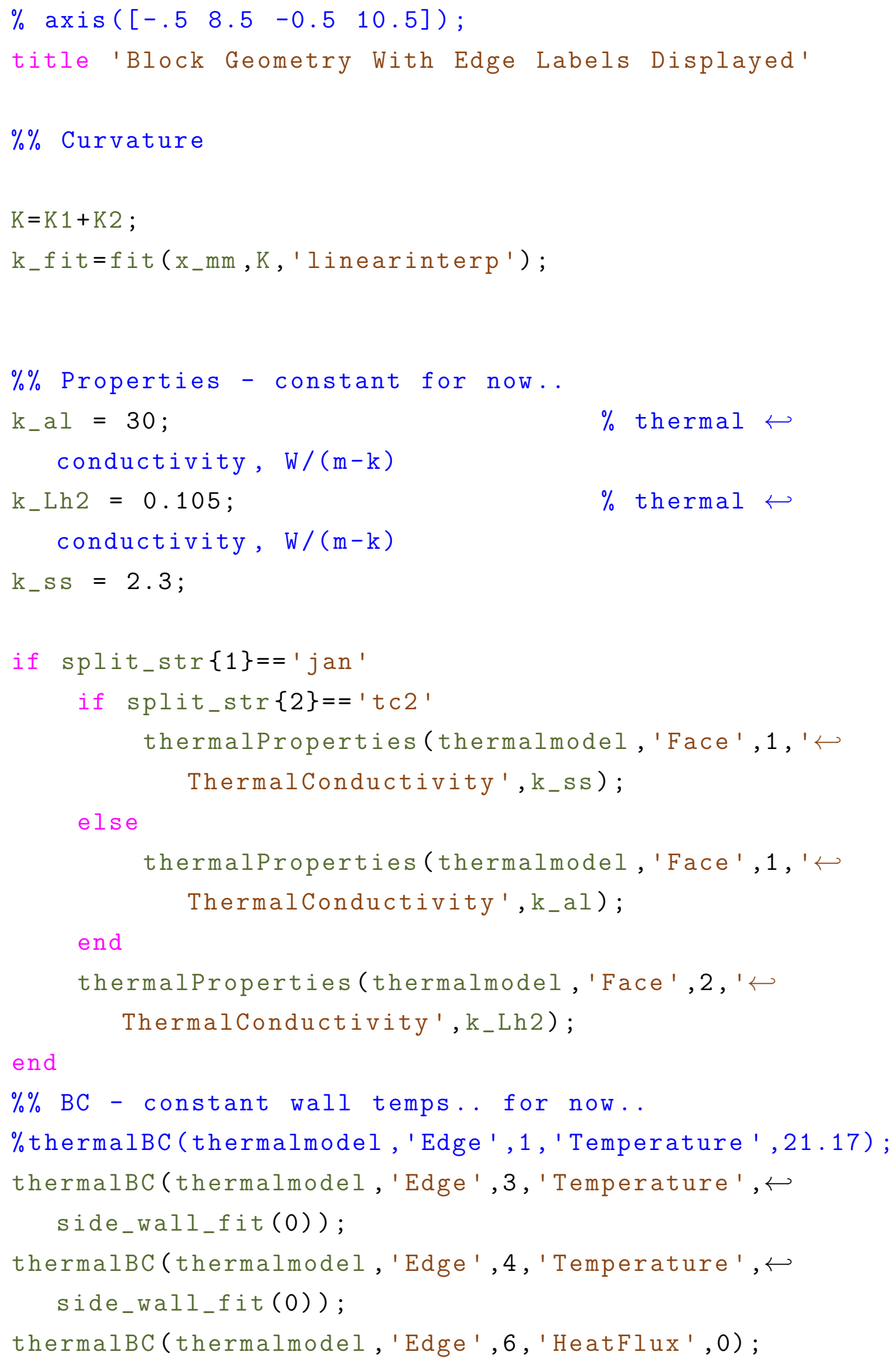




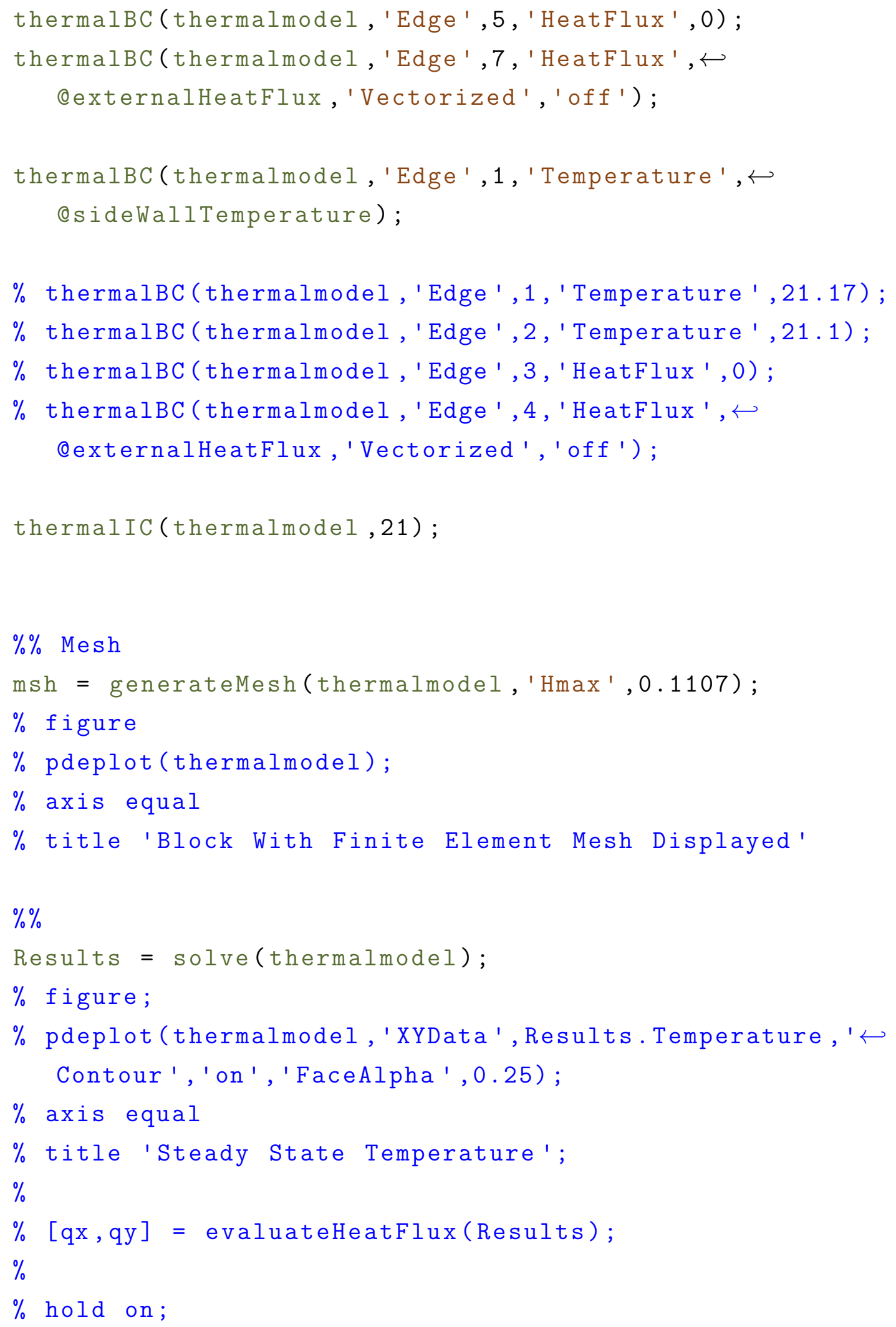


\% pdeplot(thermalmodel, 'FlowData', [qx qy])

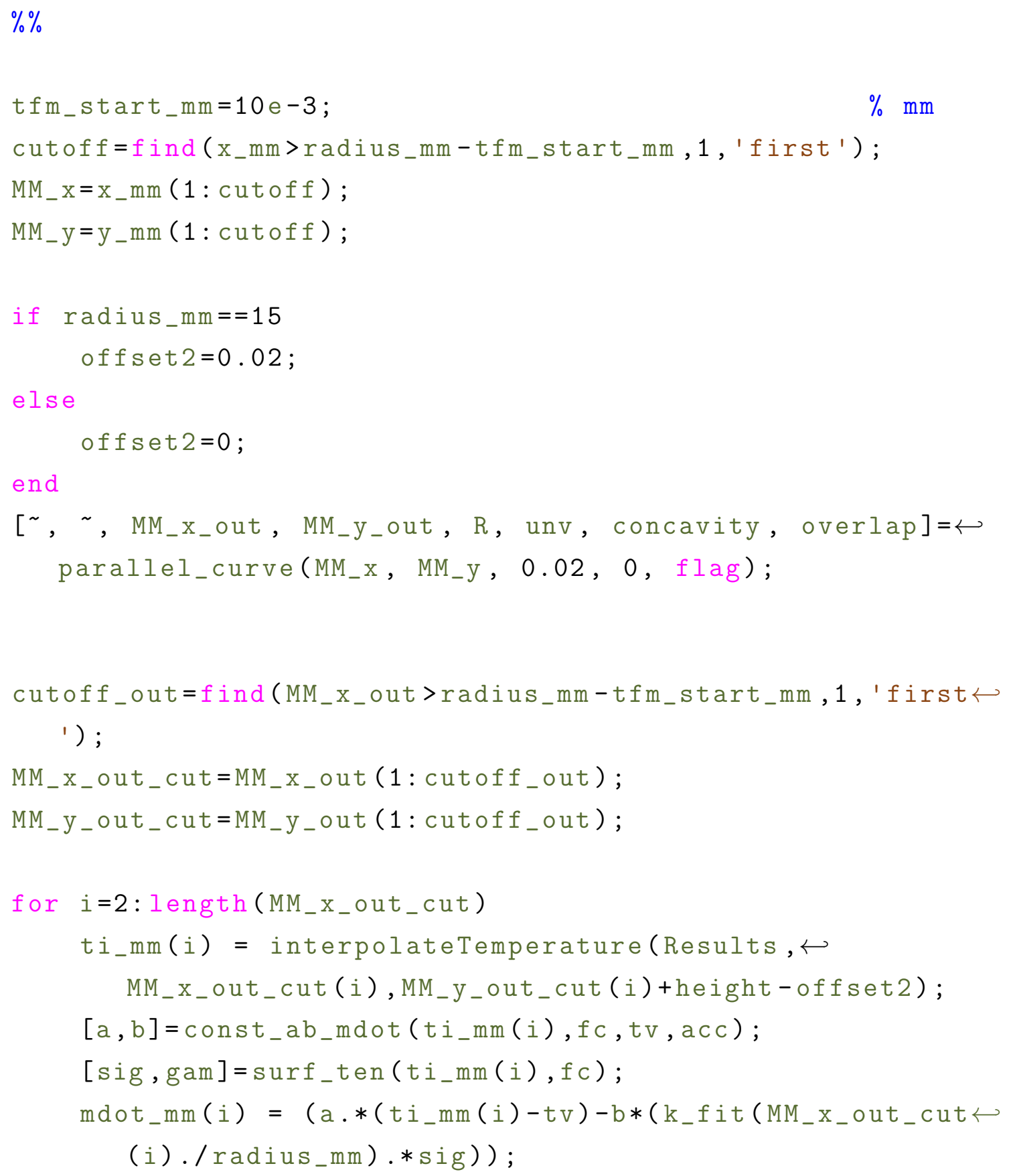




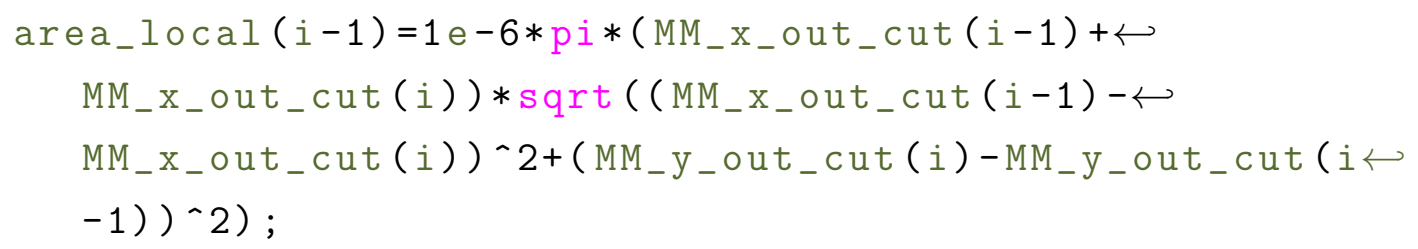

end

mdot_mm_total=nansum(mdot_mm (1: end -1$\left.). * a r e a \_l o c a l\right) ;$

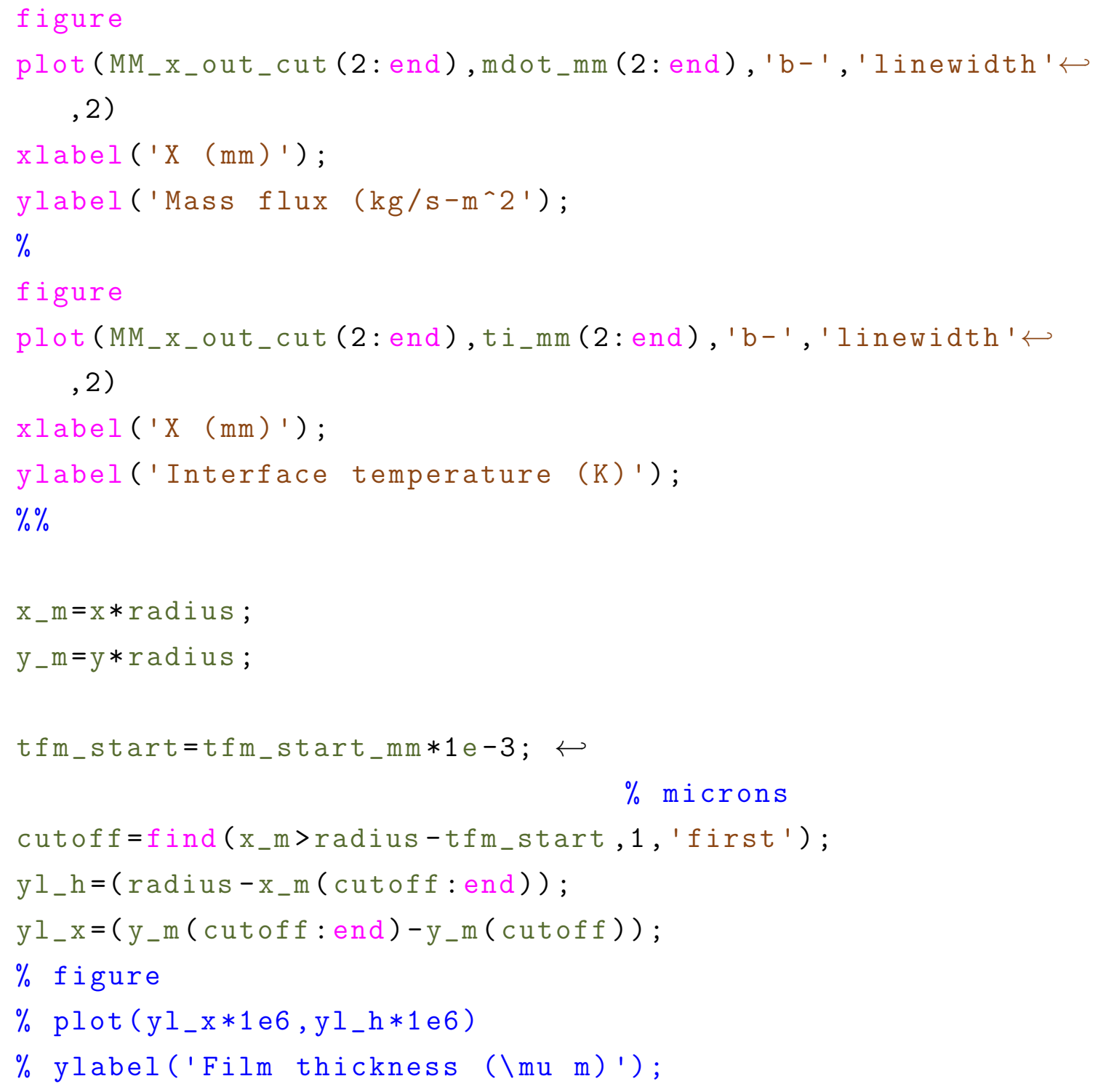




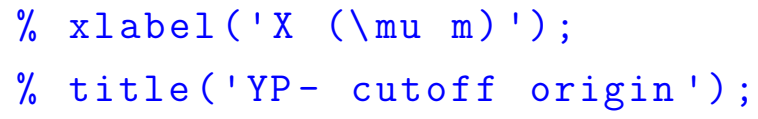

$\%$ figure

$\% \operatorname{plot}\left(t f m_{-} t w_{-} x_{-} f i t, t f m_{-} t w_{-} x_{-} c u t o f f, t f m_{-} t w_{-} t e m p\right)$ 


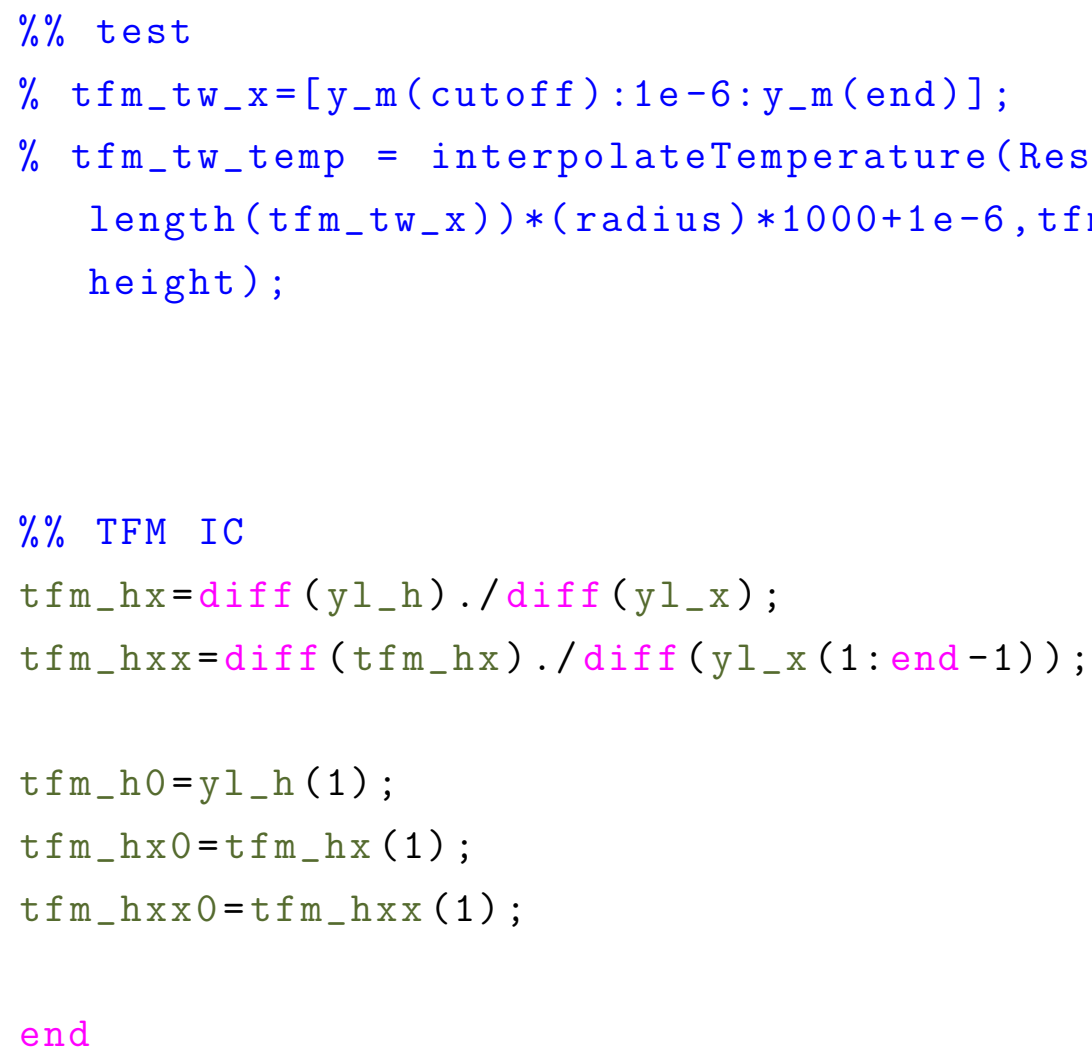

\section{B.13 sideWallTemperature.m}

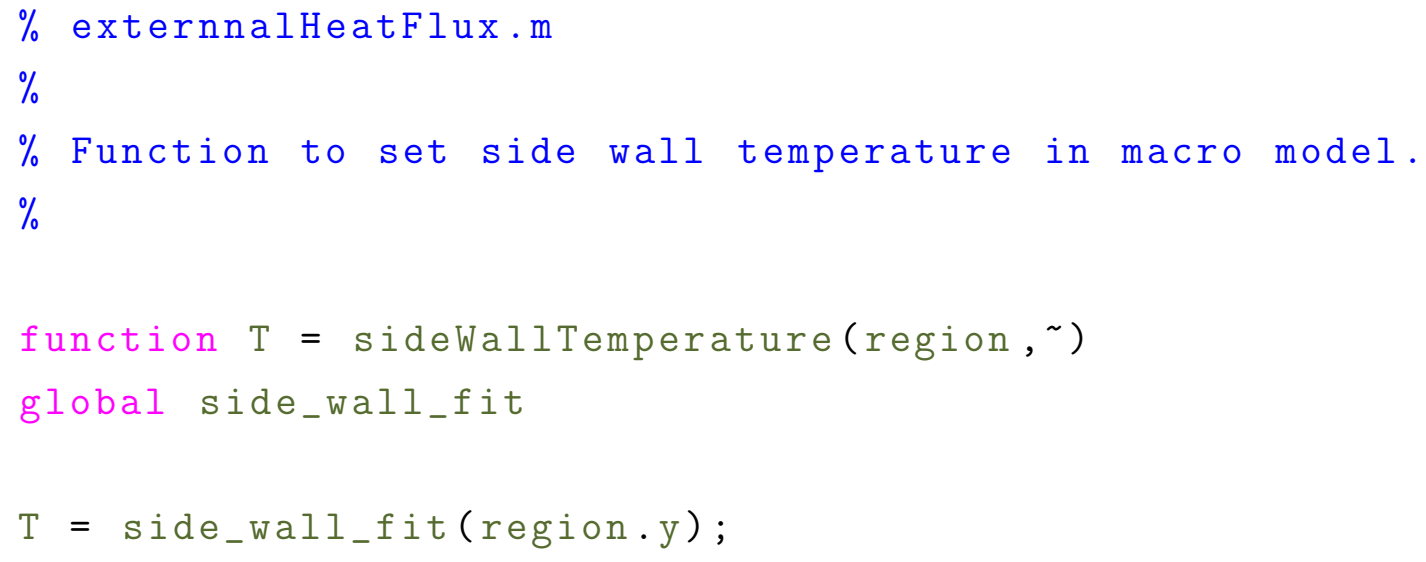


end

\section{B.14 watvisc.m}

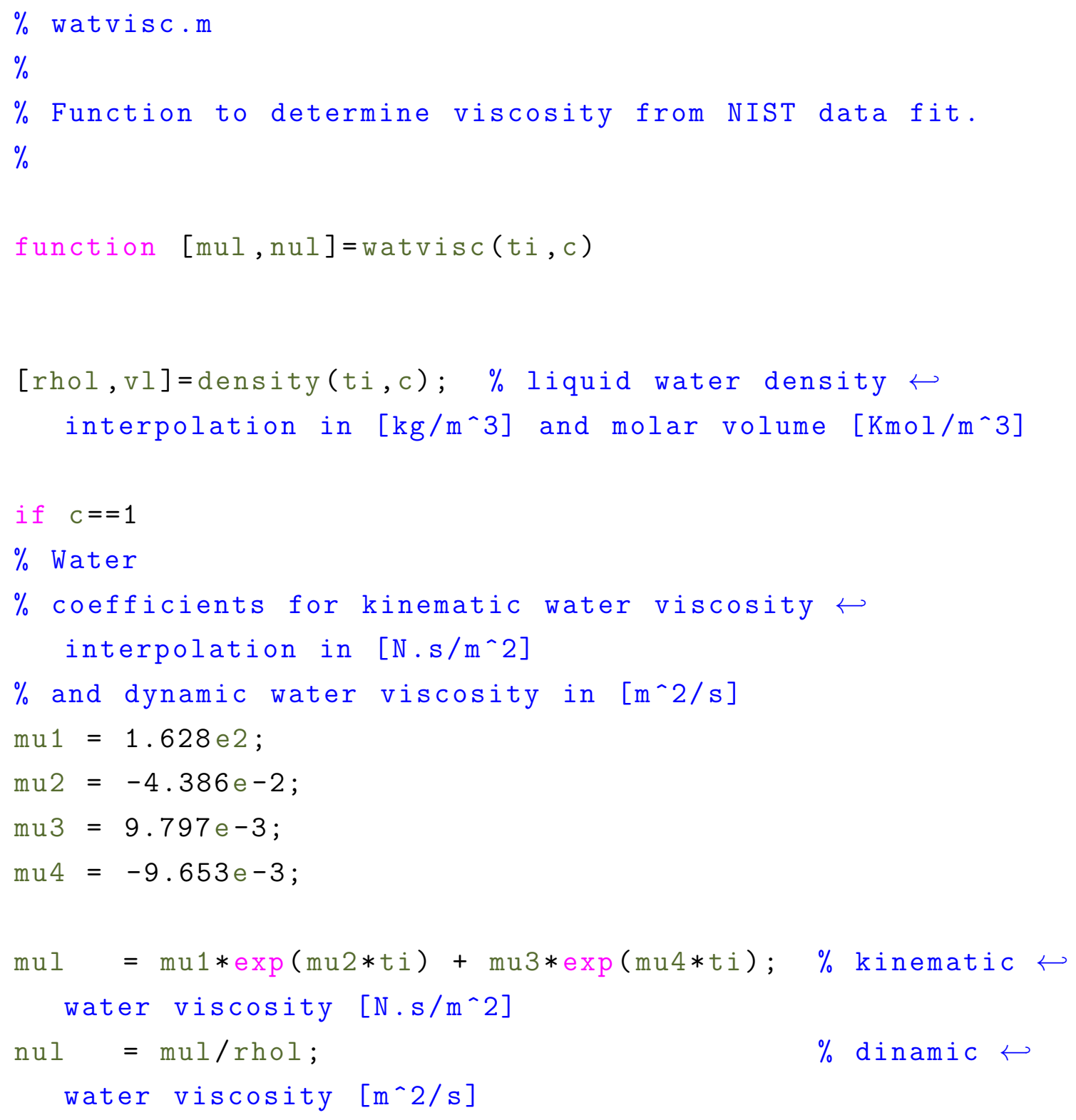




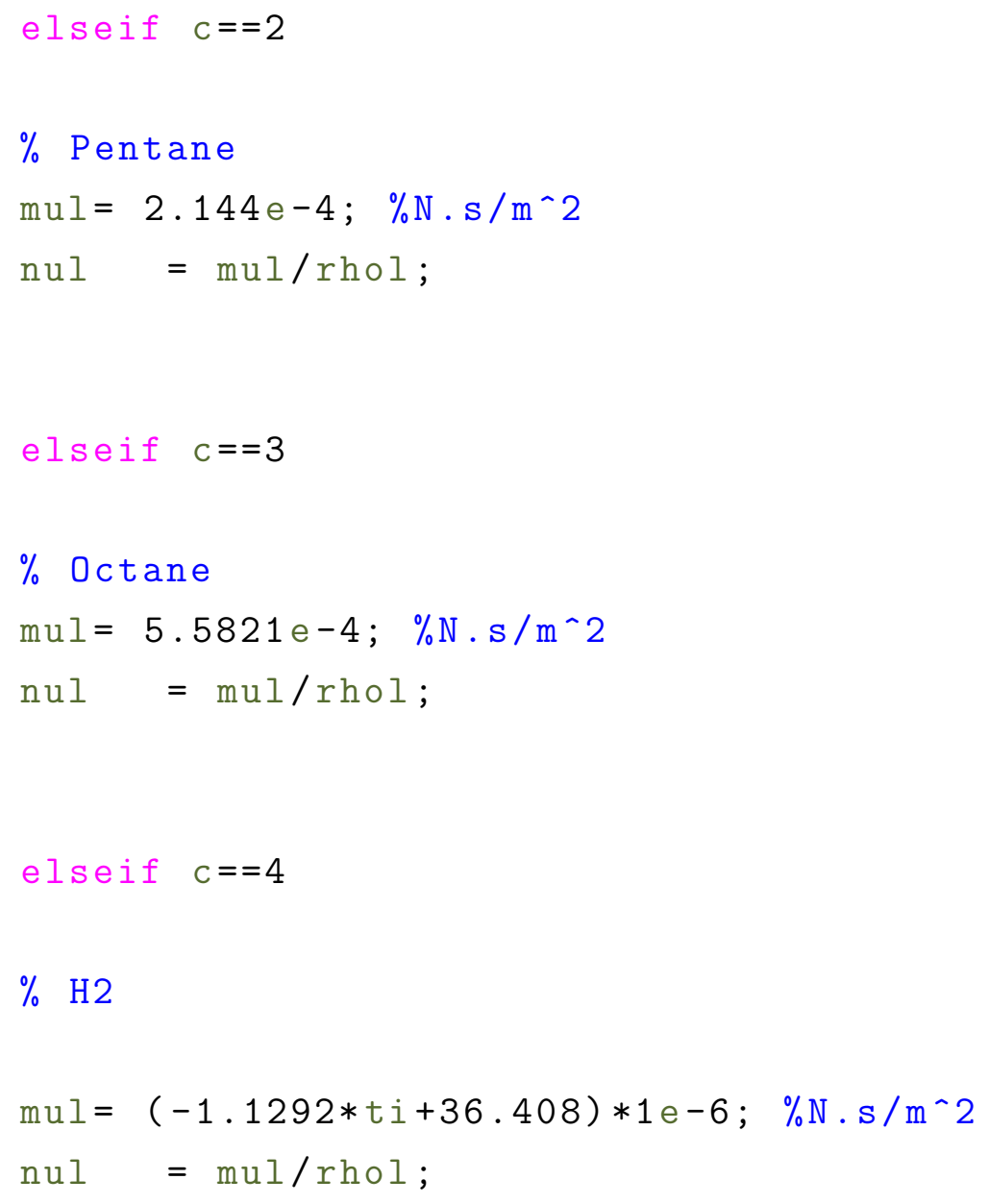

\section{B.15 surf_ten.m}

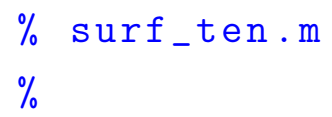




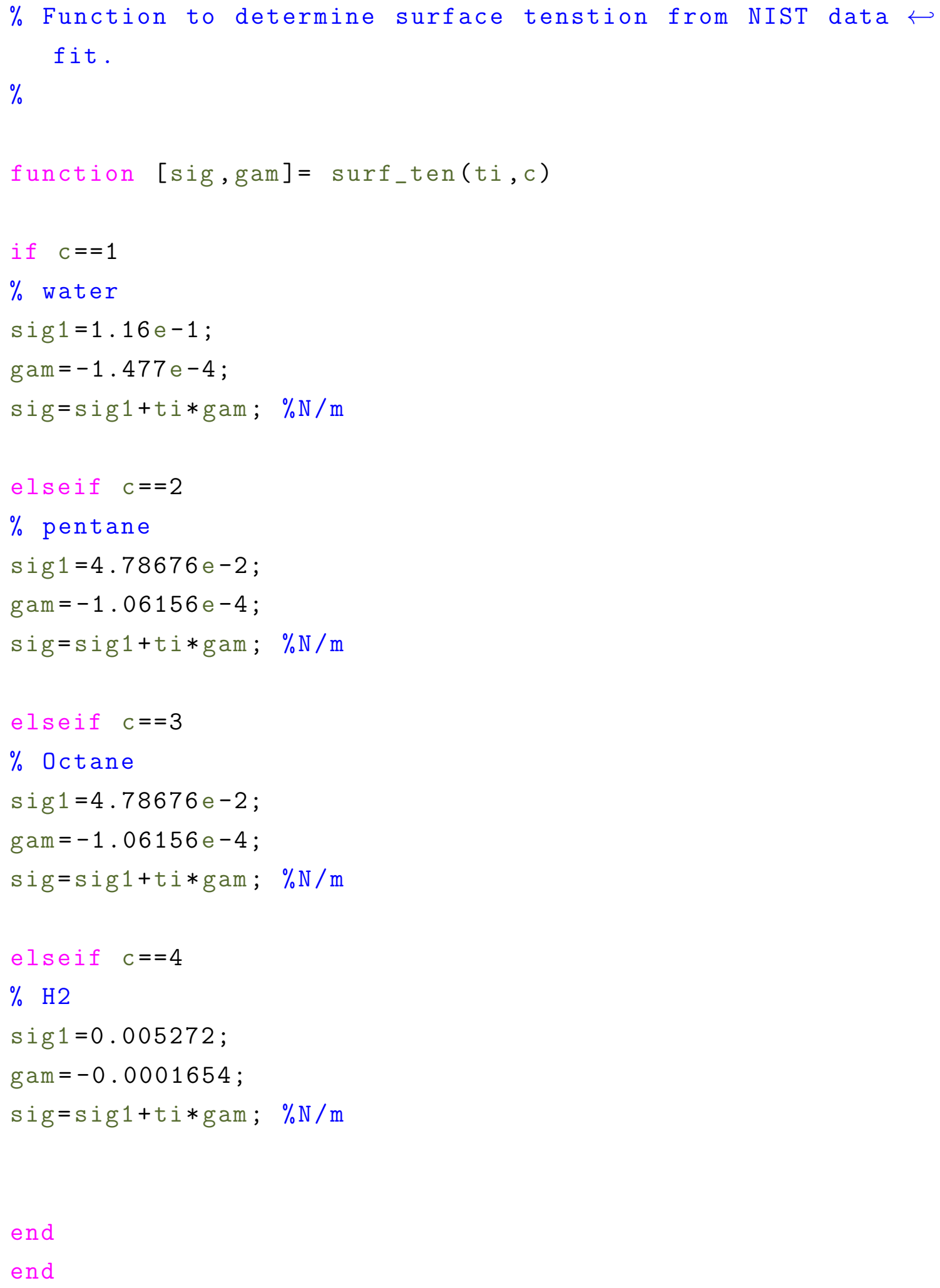

end

end 


\section{B.16 tfm_test.m}

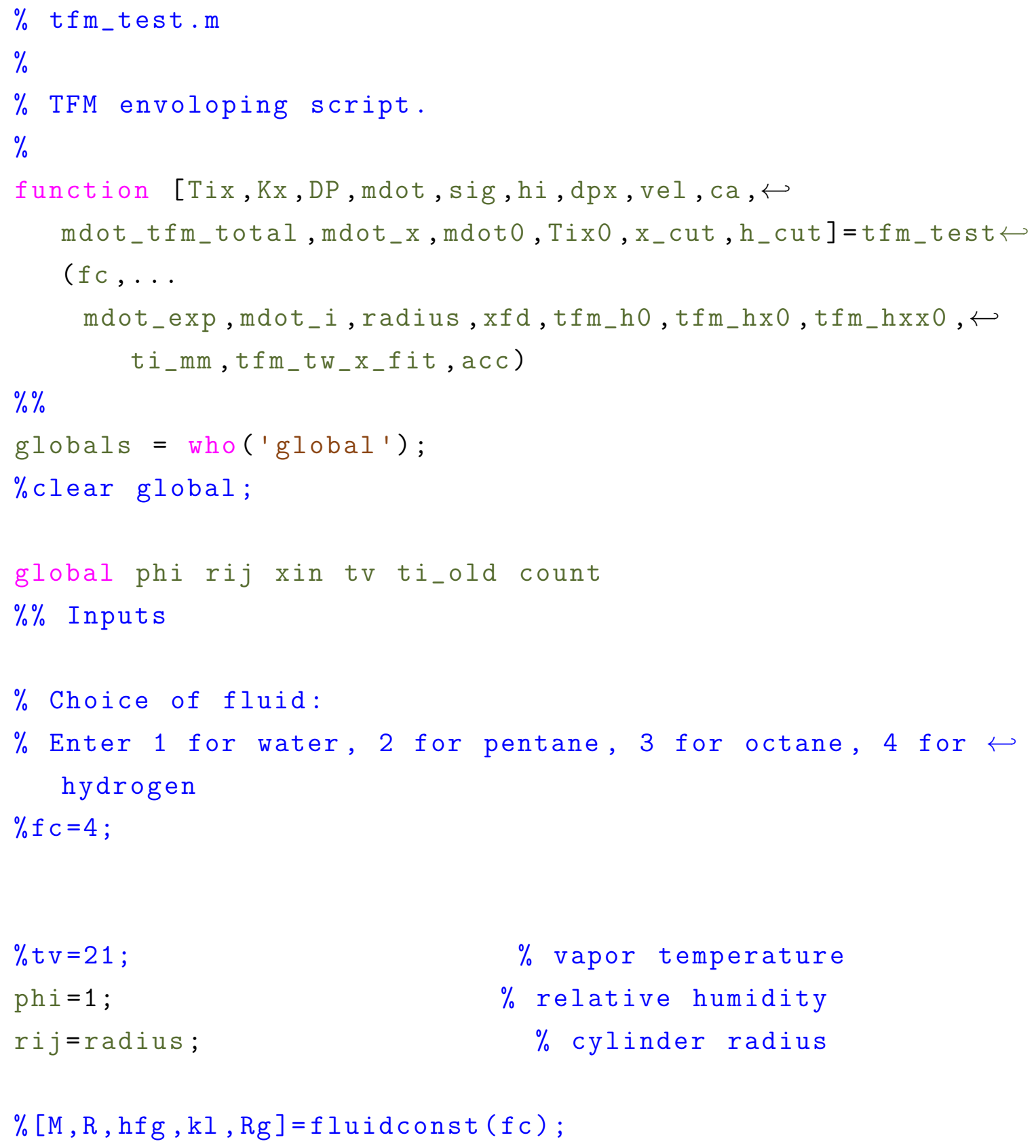




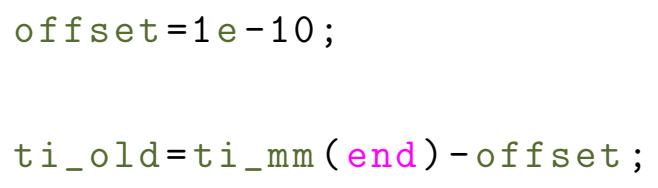

$\%$ Fixed step solver

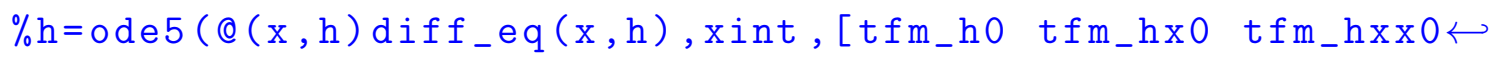
] ); 


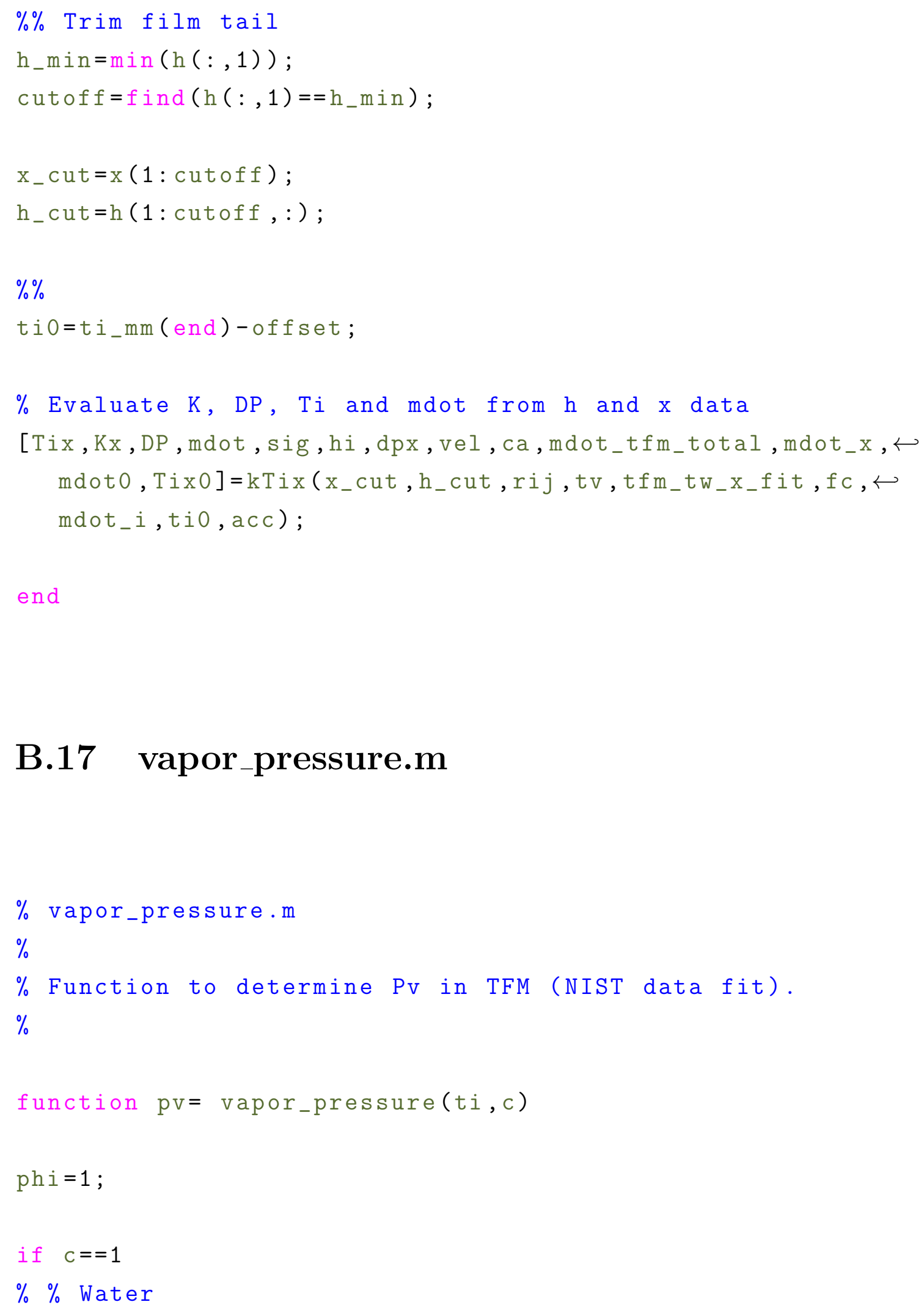

\section{B.17 vapor_pressure.m}




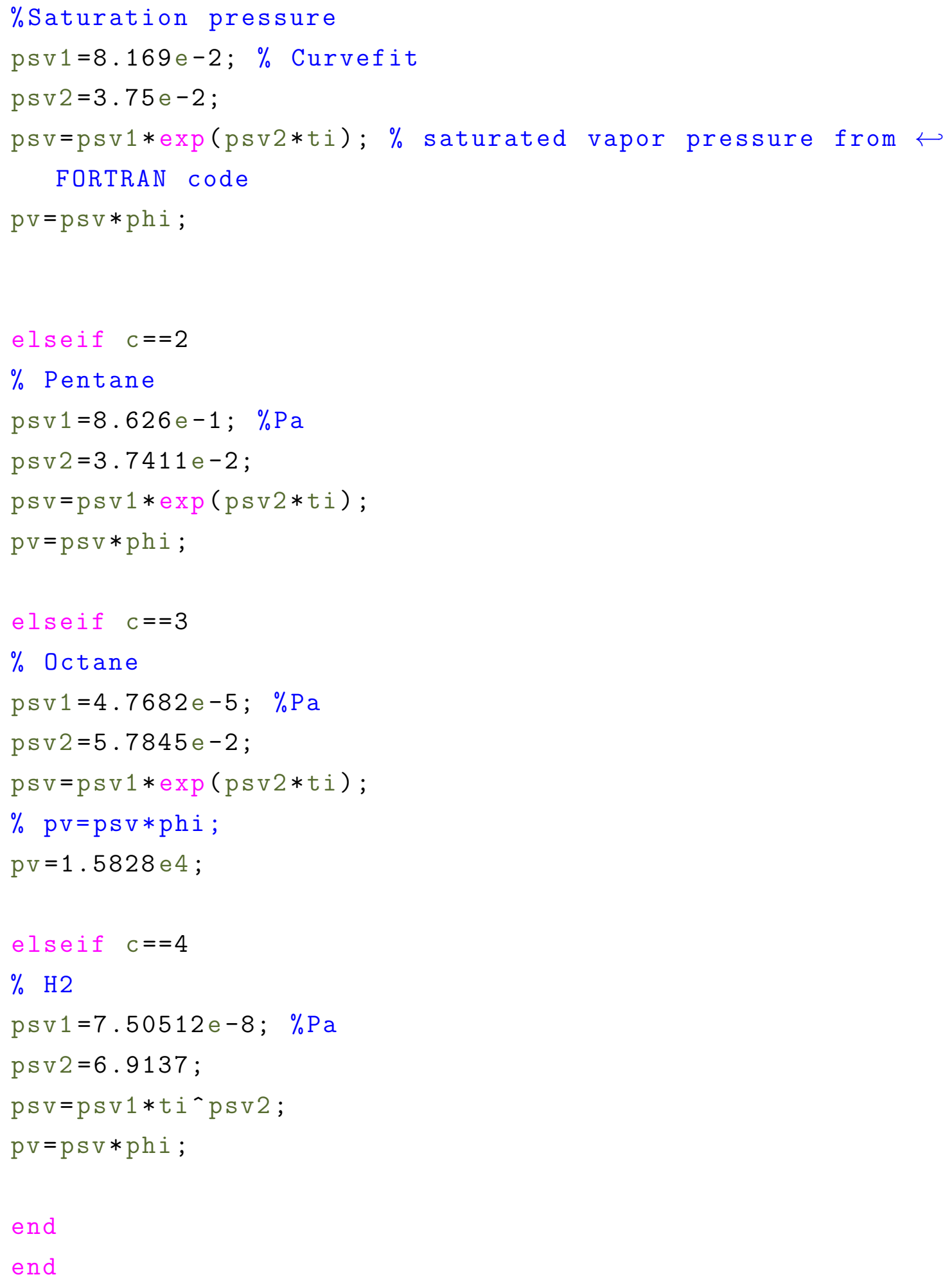

